\section{ADVANCED STEEL CONSTRUCTION}

\section{An International Journal}

Volume 6 Number 1

March 2010

CONTENTS

Technical Papers

An Improved Effective Width Method Based on the Theory of Plasticity Thomas Hansen, Jesper Gath and M.P. Nielsen

An Assessment of Beam-to-Column Endplate and Baseplate Joints Including the Axial-Moment Interaction A.A. Del Savio, D.A. Nethercot, P.C.G.S. Vellasco, L.R.O. de Lima and S.A.L. Andrade and L.F. Martha

Total Incremental Iterative Force Recovery Method and the Application in Plastic Hinge Analysis of Steel Frames

Fawu Wang and Yaopeng Liu

Experimental Study of External Diaphragm Joint Connecting CHS Column and H-Shaped Beam W.Q. Li, Y.Y. Chen, W. Wang, Y.J. Xu and X.D. Lv

Development of An Engineering Methodology for Thermal Analysis of

Protected Structural Members in Fire

Hong Liang, Stephen Welch and José L. Torero

A Specific Procedure for Seismic Design of Cold-Formed Steel Housing

R. Landolfo, L. Fiorino and O. Iuorio

Influence of Partial Loading on the Behaviour of Pallet Rack Structures F. Al Qarud, A. Shatnawi, M.S. Abdel-Jaber and R.G. Beale

Effects of Strain Regimes on the Behaviour of Headed Stud Shear Connectors for Composite Steel-Concrete Beams

O. Mirza and B. Uy

An Investigation on the Post-Local-Buckling Analysis of I-Section Struts Using Finite Strip Method H.R. Ovesy, J. Loughlan and S.A.M. Ghannadpour

Conference Announcement

Copyright $\odot 2010$ by

The Hong Kong Institute of Steel Construction

Website: $h t t p: / / w w w . h k i s c . o r g$

ISSN 1816-112X

Science Citation Index Expanded, Materials Science Citation Index and ISI Alerting

Cover: Curved Roof of the New Wuhan Bullet Train Station in Chin

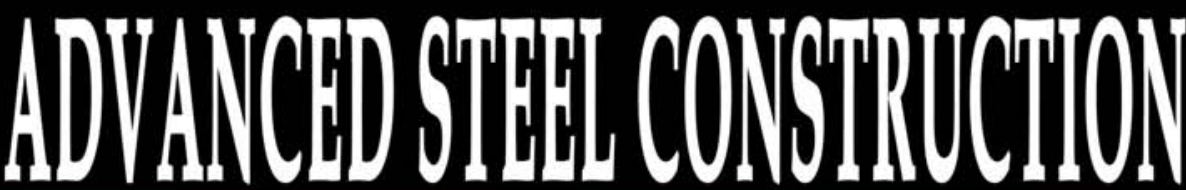

an International Jounual ISSN 1816-112X

Volume 6 Number 1

March 2010

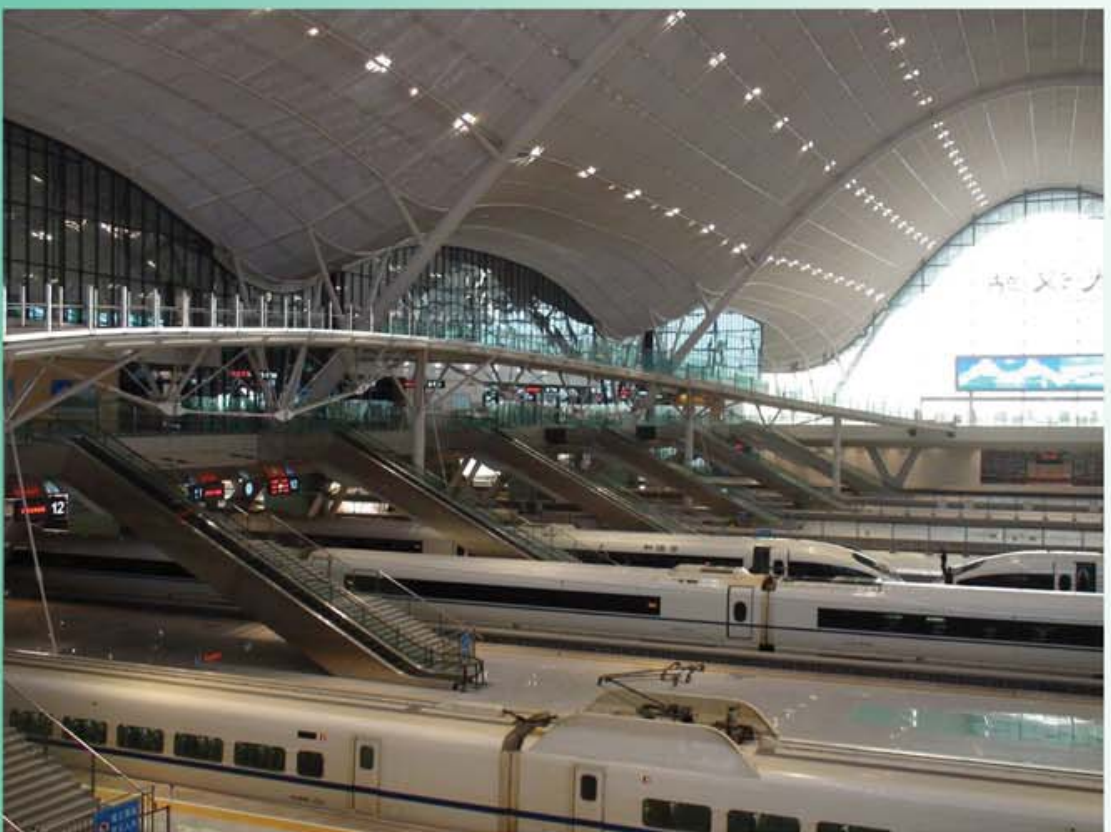

Editors-in-Chief

S.L. Chan, The Hong Kong Polytechnic University, Hong Kong

W.F. Chen, University of Hawaii at Manoa, USA

R. Zandonini, Trento University, Italy 


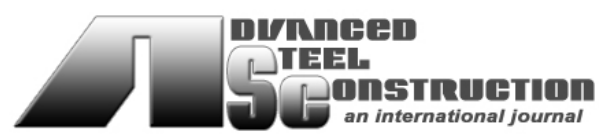

ISSN 1816-112X

Science Citation Index Expanded, Materials Science Citation Index and ISI Alerting

\section{EDITORS-IN-CHIEF}

Asian Pacific, African and organizing Editor

S.L. Chan

The Hong Kong Polyt. Univ., Hong Kong

\section{American Editor \\ W.F. Chen \\ Univ. of Hawaii at Manoa, USA}

\section{European Editor}

R. Zandonini

Trento Univ., Italy

\section{INTERNATIONAL EDITORIAL BOARD \\ F.G. Albermani}

The Univ. of Queensland, Australia

I. Burgess

Univ. of Sheffield, UK

F.S.K. Bijlaard

Delft Univ. of Technology, The Netherlands

R. Bjorhovde

The Bjorhovde Group, USA

M.A. Bradford

The Univ. of New South Wales, Australia

D. Camotim

Technical Univ. of Lisbon, Portugal

C.M. Chan

Hong Kong Uni v. of Science \& Technolog

Hong Kong

T.H.T. Chan

Queensland Univ. of Technology, Australia

S.P. Chiew

Nanyang Technological Univ., Singapore

W.K. Chow

The Hong Kong Polyt. Univ., Hong Kong

K.F. Chung

The Hong Kong Polyt. Univ., Hong Kong

G.G. Deierlein

Stanford Univ., California, USA

\section{Advanced Steel \\ Construction an international journal}

Dezi

Univ. of Ancona, Italy

D. Dubina

The Politehnica Univ. of Tim

Romania

R. Greiner

Technical Univ. of Graz, Austria

G.W.M. Ho

Ove Arup \& Pa rtners Hong Kon g Ltd. Hong Kong

B.A. Izzuddin

Imperial College of Science, Technology and Medicine, UK

J.P. Jaspart

Univ. of Liege, Belgium

S. A. Jayachandran

SERC, CSIR, Chennai, India

S. Kitipornchai

City Univ. of Hong Kong, Hong Kong

D. Lam

Univ. of Leeds, UK

G.Q. Li

Tongji Univ., China

J.Y.R. Liew

National Univ. of Singapore, Singapore

X. Liu

Tsinghua Univ., China

E.M. Lui

Syracuse Univ., USA

Y.L. Mo

Univ. of Houston, USA

$\mathrm{y}$,

CUST, Clermont Ferrand, France

D.A. Nethercot

Imperial College of Science, Technology and Medicine, UK

Y.Q. Ni

The Hong Kong Polyt. Univ., Hong Kong

D.J. Oehlers

The Univ. of Adelaide, Australia

K. Rasmussen

The Univ. of Sydney, Australia

T.M. Roberts

Cardiff Univ., UK
J.M. Rotter

The Univ. of Edinburgh, UK

C. Scawthorn

osoara, Scawthorn Porter Associates, USA

P. Schaumann

Univ. of Hannover, Germany

G.P. Shu

Southeast Univ. China

J.G. Teng

The Hong Kong Polyt. Univ., Hong Kong

G.S. Tong

Zhejiang Univ., China

K.C. Tsai

National Taiwan Univ., Taiwan

C.M. Uang

Univ. of California, USA

B. Uy

University of Western Sydney

M. Veljkovic

Univ. of Lulea, Sweden

F. Wald

Czech Technical Univ. in Prague, Czech

Y.C. Wang

The Univ. of Manchester, UK

Y.L. Xu

The Hong Kong Polyt. Univ., Hong Kong

D. White

Georgia Institute of Technology, USA

E. Yamaguchi

Kyushu Institute of Technology, Japan

Y.B. Yang

National Taiwan Univ., Taiwan

B. Young

The Univ. of Hong Kong, Hong Kong

X.L. Zhao

Monash Univ., Australia

Z.H. Zhou

Alpha Consultant Ltd., Hong Kong 


\begin{tabular}{ll}
\hline \hline & General Information \\
& Advanced Steel Construction, an international journal \\
\hline \hline Aims and scope
\end{tabular}

\section{Aims and scope}

The International Journal of Advanced Steel Construction provides a platform for the publication and rapid dissemination of ori ginal and up-to-date research and tec hnological developments in steel c onstruction, design and anal ysis. Scope of research $p$ apers published in this journal includes but is not limite $d$ to theor etical and expe rimental research on elements, assemblages, sy stems, material, design philosophy and codification, standards, fabrication, projects of innov ative nature an d computer tech niques. The journal is specifically $t$ ailored to channel the e xchange of tec hnological know-ho $w$ bet ween $r$ esearchers an d practitioners. Contributions from all aspects related to the recent developments of advanced steel construction are welcome.

\section{Instructions to authors}

Submission of the manuscript. Authors may submit double-spaced manuscripts preferably in MS Word by emailing to one of the chief editors as follows for arrangement of review. Alternatively papers can be submitted on a diskette to one of the chief editors.

Asian Pacific, African and organizing editor: Professor S.L. Chan, Email: ceslchan@polyu.edu.hk

American editor :

European editor:

Professor S.L. Chan, Email: ceslchan@polyu.edu

Professor R. Zandonini, Email: riccardo_zandonini@ing.unitn.it

All manuscripts submitted to the journal are recommended to accompany with a li st of four potential reviewers suggested by the author(s). This list should include the complete name, add ress, telephone and fax num bers, em ail address, and at least five keywords that identify the expertise of each reviewer. This scheme will improve the process of review.

Style of manuscript

General. Author(s) should provide full postal and email addresses and fax number for correspondence. The manuscript including abstract, keywords, references, figures and tables should be in English with pages numbered and typed with double line spacing on single side of A4 or letter-sized paper. The front page of the article should contain:

a) a short title (reflecting the content of the paper);

b) all the name(s) and postal and email addresses of author(s) specifying the author to whom correspondence and proofs should be sent;

c) an abstract of $100-200$ words; and

d) 5 to 8 keywords.

The paper must contain an introduction and a conclusion. The length of paper should not exceed 25 journal pages (approximately 15,000 words equivalents).

Tables and figures. Tables and figures including photographs should be typed, numbered consecutively in Arabic numerals and with short titles. They should be referred in the text as Figure 1, Table 2, etc. Originally drawn figures and photographs should be provided in a form suitable for photographic reproduction and reduction in the journal.

Mathematical expressions and units. The Systeme Internationale (SI) should be followed whenever possible. The numbers identifying the displayed mathematical expression should be referred to in the text as Eq. (1), Eq. (2).

References. References to published literature should be referred in the text, in the order of citation with Arabic numerals, by the last name(s) of the author(s) (e.g. Zandonini and Zanon [3]) or if more than three authors (e.g. Zandonini et al. [4]). References should be in English $w$ ith occasional allow ance of 1-2 e xceptional referenc es in local lang uages and $r$ eflect the curren $t$ state-of-technology. Journal titles should be abbreviated in the style of the Word List of Scientific Periodicals. References should be cited in the following style $[1,2,3]$.

Journal: [1] Chen, W.F. and Kishi, N., "Semi- rigid Steel Beam-to-column Connections, Data Base and Modellin g", Journal of Structural Engineering, ASCE, 1989, Vol. 115, No. 1, pp. 105-119.

Book: [2] Chan, S.L. and Chui, P.P.T., "Non-linear Static and Cyclic Analysis of Semi-rigid Steel Frames", Elsevier Science, 2000 .

Proceedings: [3] Zandonini, R. a nd Zanon, P., "Experimental Analy sis of S teel Beams with Semi -rigid Joint s", Proceedings of International Conference on Advances in Steel Structures, Hong Kong, 1996, Vol. 1, pp. 356-364.

Proofs. Proof will be sent to the c orresponding author to correct an y typesetting errors. Alternations to the original manuscript at this stage will not be accepted. Proofs should be returned within 48 hours of receipt by Express Mail, Fax or Email.

Copyright. Submission of an article to "Advanced Steel Construction" implies that it presents the original and unpublished work, and not under consideration for publication nor published elsewhere. On acceptance of a manuscript submitted, the copyright thereof is transferred to the publisher $b y$ the Transfer of $C$ opyright Agreement and upon $t$ he acceptance of publication for the $p$ apers, the corresponding author must sign the form for Transfer of Copyright.

Permission. Quoting from this journal is granted provided that the customary acknowledgement is given to the source.

Page charge and Reprints. There will be no page charges if the length of paper is within the limit of 25 journal pages. A total of 30 free offprints will be supplied free of charge to the corresponding author. Purchasing orders for additional offprints can be made on order forms which will be sent to the authors. These instructions can be obtained at the Hong Kong Institute of Steel Construction, Journal website: http://www.hkisc.org

The International Journal of Advanced Steel Construction is published quarterly by non-profit making learnt society, The Hong Kong Institute of Steel Construction, c/o Department of Civil \& Structural Engineering, The Hong Kong Polytechnic University, Hung Hom, Kowloon, Hong Kong.

Disclaimer. No responsibility is assumed for a ny injury and / or damage to per sons or property as a matter of products liability, negligence or otherwise, or from any use or operation of any methods, products, instructions or ideas contained in the material herein.

Subscription inquiries and change of address. Address all subscription inquiries and correspondence to Member Records, IJASC. Notify an address change as soon as possible. All communications should include both old and new addresses with zip codes and be accompanied by a mailing label from a recent issue. Allow six weeks for all changes to become effective.

The Hong Kong Institute of Steel Construction

HKISC

c/o Department of Civil and Structural Engineering,

The Hong Kong Polytechnic University,

Hunghom, Kowloon, Hong Kong, China.

Tel: 852- 27666047 Fax: 852- 23346389

Email: ces/chan@polyu.edu.hk Website: http://www.hkisc.org/

ISSN 1816-112X

Science Citation Index Expanded, Materials Science Citation Index and ISI Alerting

Copyright $\odot 2010$ by:

The Hong Kong Institute of Steel Construction. 


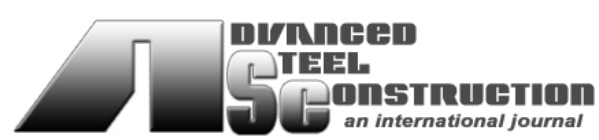

ISSN 1816-112X

Science Citation Index Expanded, Materials Science Citation Index and ISI Alerting

\section{EDITORS-IN-CHIEF}

Asian Pacific, African and organizing Editor

S.L. Chan

The Hong Kong Polyt. Univ., Hong Kong

Email: ceslchan@polyu.edu.hk

\section{American Editor}

W.F. Chen

Univ. of Hawaii at Manoa, USA

Email:waifah@hawaii.edu

\section{European Editor}

R. Zandonini

Trento Univ., Italy

Email: riccardo.zandonini@ing.unitn.it

\section{Advanced Steel \\ Construction an international journal}

VOLUME 6 NUMBER 1

MARCH 2010

Technical Papers

An Improved Effective Width Method Based on the

Theory of Plasticity

Thomas Hansen, Jesper Gath and M.P. Nielsen

An Assessment of Beam-to-Column Endplate and

Baseplate Joints Including the Axial-Moment Interaction

A.A. Del Savio, D.A. Nethercot, P.C.G.S. Vellasco,

L.R.O. de Lima and S.A.L. Andrade and L.F. Martha

Total Incremental Iterative Force Recovery Method and

the Application in Plastic Hinge Analysis of Steel Frames

Fawu Wang and Yaopeng Liu

Experimental Study of External Diaphragm Joint Connecting

CHS Column and H-Shaped Beam

W.Q. Li, Y.Y. Chen, W. Wang, Y.J. Xu and X.D. Lv

Development of An Engineering Methodology for

Thermal Analysis of Protected Structural Members in Fire

Hong Liang, Stephen Welch and José L. Torero

A Specific Procedure for Seismic Design of

Cold-Formed Steel Housing

R. Landolfo, L. Fiorino and O. Iuorio

Influence of Partial Loading on the Behaviour of

Pallet Rack Structures

F. Al Qarud, A. Shatnawi, M.S. Abdel-Jaber and R.G. Beale

Effects of Strain Regimes on the Behaviour of Headed

Stud Shear Connectors for Composite Steel-Concrete Beams

O. Mirza and B. Uy

An Investigation on the Post-Local-Buckling Analysis of I-Section Struts Using Finite Strip Method

H.R. Ovesy, J. Loughlan and S.A.M. Ghannadpour

662

Conference Announcement 


\title{
AN IMPROVED EFFECTIVE WIDTH METHOD BASED ON THE THEORY OF PLASTICITY
}

\author{
Thomas Hansen *, Jesper Gath and M.P. Nielsen \\ ALECTIA A/S, Teknikerbyen 34, DK-2830 Virum, Denmark \\ *(Corresponding author: E-mail: tmh@alectia.com)
}

Received: 11 May 2007; Revised: 17 July 2008; Accepted: 17 September 2008

\begin{abstract}
Currently, calculations of plates in compression are based on the semi-empirical effective width method which was developed by Winter et al.

The effective width method takes the post-buckling capacity into account. The aim of the paper is to establish an effective width method, which is derived on the basis of a consistent theory. The method rests on the theory of plasticity, particularly the yield line theory. Emphasis is on buckling problems related to plate girders. Two general cases are studied: Plates in uniaxial compression supported along all edges, cf. the compressed flange in a box girder, and plates with one free edge, cf. the compressed flange and the transverse web stiffeners in an I-shaped girder.

The results presented coincide closely with Winter's formulae and with tests.
\end{abstract}

Keywords: Post-buckling, thin plates, theory of plasticity, yield line theory, effective width method, failure mechanisms, stability, in-plane loading

\section{INTRODUCTION}

It was deduced many years ago that the elastic buckling theory is not able to accurately account for the real strength of plates (Schuman and Back [12]). The main reason for this is that, in a large parameter interval, the ultimate load is reached after yielding of the plate. This fact was also pointed out by Kármán et al. [8], who suggested modifying the elastic solution by an empirical coefficient. This idea was taken up by Winter [15], who developed accurate formulae for the two important cases considered in the following. Winter's method is an the effective width method, on which calculations of plates in compression are still based.

Any design method thus has to take into account that plates in compression may carry loads much larger than the load for which elastic buckling will occur.

The aim is here to establish an effective width method, which is derived on the basis of a consistent theory. The method rests on the theory of plasticity, particularly the yield line theory. The new constribution of the paper lies in the way the yield line theory is applied on the deflected shape of the plate. The emphasis is attached to buckling problems related to plate girders. Two general cases are studied: Plates in uniaxial compression supported along all edges, cf. the compressed flange in a box girder, and plates with one free edge, cf. the compressed flange and the transverse web stiffeners in an I-shaped girder.

The basics of the theory are described in Chapter 2. How to take geometrical second order effects into account is illustrated by a column example.

Solutions for plates in uniaxial compression supported along all edges are presented in Chapter 3, while solutions for plates with one free edge are treated in Chapter 4. Both chapters contain a comparison between the theory and the experimental results, on which Winter's formulae were based. 
The effect of imperfections is touched upon in Chapter 5 and other possible applications of the theory are mentioned in Chapter 6.

\subsection{Historical Overview}

Bryan [2] was the first to develop a solution for the elastic critical stress of a rectangular plate simply supported along all edges and subjected to a uniform longitudinal compressive stress. Later a large number of solutions using Bryan's equation have been derived, see for instance (Timoshenko and Gere [14]).

In general the elastic critical stress may be expressed as

$$
\sigma_{c r}=k \frac{\pi^{2} E}{12\left(1-v^{2}\right)}\left(\frac{t}{b}\right)^{2}
$$

in which $E$ is Young's modulus, $v$ Poisson's ratio, $t / b$ the thickness-to-width ratio and $k$ is a buckling coefficient, which is a function of plate geometry and boundary conditions. Useful information on $k$-factors may be found in a number of references, e.g. (Timoshenko and Gere [14]).

Tests by Schuman and Back [12] on plates supported by V-grooves along the unloaded edges demonstrated that, for plates of the same thickness, an enhancement of the plate width beyond a certain value did not increase the ultimate load. Wider plates acted as though narrow side portions or "effective load-carrying areas" took most of the load. Furthermore, the ultimate load was found to be up to thirty times larger than the elastic critical buckling load determined by Bryan's equation, cf. Eq. 1.

It is now well known that the post-buckling resistance of plates is due to redistribution of axial compressive stresses, and to a lesser extent, to tensile membrane effects and to shear that accompany the out-of-plane bending of the plate in both longitudinal and transverse directions. The longitudinal stresses tend to concentrate in the vicinity of the longitudinally supported edges, which are the stiffer parts of the buckled plate. As a result, yielding begins along these edges, which limits the load-carrying capacity.

Several researchers have been prompted by the tests of Schuman and Back [12] to develop expressions for the ultimate strength of such plates. The first to use the effective width concept in handling this problem was Kármán et al. [8]. They derived the following approximate formula for the effective width, $b_{e}$, of plates supported along all edges, based on the assumption that two strips with total width, $b_{e}$, along the sides, each on the verge of buckling, carry the entire load:

$$
b_{e}=\frac{\pi}{\sqrt{3\left(1-v^{2}\right)}} t \sqrt{\frac{E}{\sigma_{e}}}
$$

Here $\sigma_{e}$ is the edge stress along $b_{e}$ and the remaining notation is as in Eq. 1.

As a result of many tests and studies of post-buckling strength, Winter [15] suggested the following formula for the effective width:

$$
b_{e}=1.9 t \sqrt{\frac{E}{\sigma_{e}}}\left(1.0-0.574 \frac{t}{b} \sqrt{\frac{E}{\sigma_{e}}}\right)
$$


This equation has been modified several times over the past. In the latest version, adopted in Eurocode for steel structures, EC3 [4], the formula is written as

$$
\frac{b_{e}}{b}=\sqrt{\frac{\sigma_{c r}}{\sigma_{e}}}\left(1.0-0.22 \sqrt{\frac{\sigma_{c r}}{\sigma_{e}}}\right)
$$

In the calculation of the ultimate compression load, the edge stress, $\sigma_{e}$, is taken to be equal to the yield stress of the plate material. The elastic critical stress, $\sigma_{c r}$, is determined by Eq. 1. Eq. 4 corresponds to Eq. 3 if the factor 0.574 is substituted with a factor of 0.415 .

For plates supported along only one longitudinal edge, the effective width has been experimentally determined by Winter [15]. The original formula was

$$
b_{e}=1.25 t \sqrt{\frac{E}{\sigma_{e}}}\left(1.0-0.333 \frac{t}{b} \sqrt{\frac{E}{\sigma_{e}}}\right)
$$

This equation has also been modified several times. In the latest version, adopted in EC3 [4], it is written as

$$
\frac{b_{e}}{b}=\sqrt{\frac{\sigma_{c r}}{\sigma_{e}}}\left(1.0-0.188 \sqrt{\frac{\sigma_{c r}}{\sigma_{e}}}\right)
$$

In the 1993-edition of EC3 [3], Eq. 4 referred to the case of plates supported along all edges and plates with one free edge. Hence, the difference between the two cases is only the value of the buckling coefficient, $k$. It is practical and convenient to have only one expression. The reason for changing the factor 0.22 to 0.188 , for plates with one free edge, is as yet not know to the authors. But the adjustment seems to be rather insignificant.

\section{POST-BUCKLING THEORY FOR PLATES IN COMPRESSION}

The basic idea is to use plastic theory in the form of limit analysis on the deflected shape of the plate. The deflected shape has to be estimated which is done by using simple formulae from beam and plate theory. The plastic analysis is carried out using yield line theory. Hence, strain hardening is not taken into consideration. Furthermore, the effect of residual stresses is not considered since, according to the theory of plasticity, they have no influence on the load-carrying capacity. Imperfections may easily be taken into account if it is assumed that the imperfections have the same form as the deflected shape of the plate at maximum load.

The contribution of the paper lies in the way the yield line theory is applied on the deflected shape of the plate. Since the deflected shape has to be estimated, the application of the method requires qualified engineering judgement.

\subsection{Yield Line Theory}

Yield line theory is an upper-bound method. The mechanisms considered are a system of bending yield hinges along lines, the yield lines. The load-carrying capacity is determined by the work equation, equalising external work and dissipation in the yield lines. 
It is assumed that the plane stress field existing before buckling is known so that the principal normal forces may be found whereby the corresponding yield moments may be determined. In an unloaded direction, the bending capacity, $m$, reaches the full yield moment per unit length in bending, $m_{p}$ :

$m=m_{p}=\frac{1}{4} t^{2} f_{y}$

where $t$ is the thickness of the plate and $f_{y}$ the yield stress. In the direction of a principal compression or tension (normal force $n$ per unit length), the bending capacity, $m$, is reduced due to the normal force as for beams subjected to combined bending and normal force, i.e.

$$
m=m_{p}\left(1-\left(\frac{n}{n_{p}}\right)^{2}\right)
$$

where $m_{p}$ is given by Eq. 7 and $n_{p}=t f_{y}$ is the load-carrying capacity in pure compression or tension. By determining the yield moments in the two principal directions in this way, the simplification suggested by Johansen [6] may be used to calculate the bending moment in a yield line, $m_{b}$, as

$$
m_{b}=m_{p x} \sin ^{2} \beta+m_{p y} \cos ^{2} \beta
$$

where $\beta$ is the angle between the yield line and the $x$-axis. The $x$-and $y$-axes are directed along the principal directions, and $m_{p x}$ and $m_{p y}$ are the corresponding yield moments per unit length. The plastic yield moments, $m_{p x}$ and $m_{p y}$, are determined by either Eq. 7 or Eq. 8 . Eq. 9 is correct for reinforced concrete slabs in general, which was shown by Nielsen [11]. It is also correct if the yield condition in principal moment space is square or rectangular. For steel plates, such assumptions are dubious, when the slab is acted upon by torsion. Nevertheless, Eq. 9 is used in the following with surprisingly good results.

A number of different researchers have developed formulae for the plastic moment capacity of inclined yield lines, the first being Murray [10]. Hiriyur and Schafer [5] and Zhao [16] have shown that the solutions obtained by the different proposals vary widely, and Zhao [16] concludes that nothing better than the method suggested by Murray [10] has been found. The present paper aims at improving this state of affairs.

\subsection{Effect of Deflections}

From the general theory of beam-columns, it is known that the equilibrium equations may be derived for the undeformed structure if a fictitious load is included. The fictitious load is a moment per unit length of the beam, equal to

$m=N \frac{d u}{d x}$

where $N$ is normal force and $u$ the deflection, transverse to the beam axis, $x$. The statical equivalence of $m$ may be expressed in several ways, but for a given part of a beam subjected to a constant normal force, $N$, it may conveniently be expressed in the following simple way: The total moment, $M$, on a beam $(A-B)$, when $N$ is constant, is 


$$
\begin{aligned}
M & =\int_{A}^{B} m d x=\int_{A}^{B} N \frac{d u}{d x} d x=N \int_{A}^{B} \frac{d u}{d x} d x=N\left(u_{B}-u_{A}\right) \\
& =N \Delta u
\end{aligned}
$$

which may be split into two forces, $N \Delta u / L$, in the two end points. The two forces are transverse to the beam as shown in Figure 1.

The value of these forces is independent of the deflected shape between the end points $(A$ and $B)$. Only the difference in the deflection at the end points is important. The work in a virtual displacement, where the beam considered moves as a rigid body, may then be determined as the work done by the two forces, $N \Delta u / L$.

In the simple cases considered in the present paper, the above result may be used by considering the plate as being subdivided into strips with constant normal force. In Figure 2, a simply supported square plate subjected to uniaxial compression is shown.

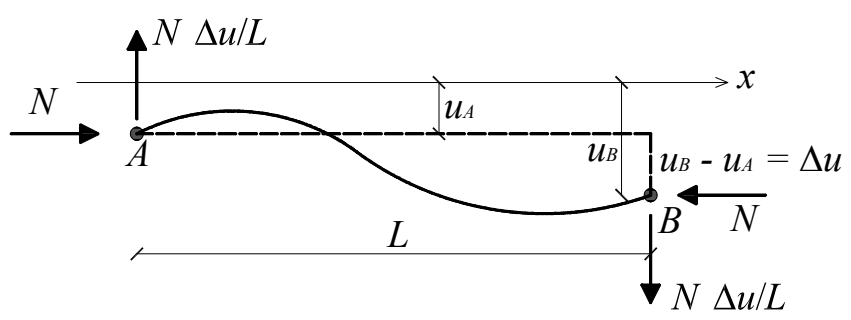

Figure 1. Part of a Beam Subjected to a Constant Normal Force, $N$, and Statically Equivalent Transverse Forces in the End Points

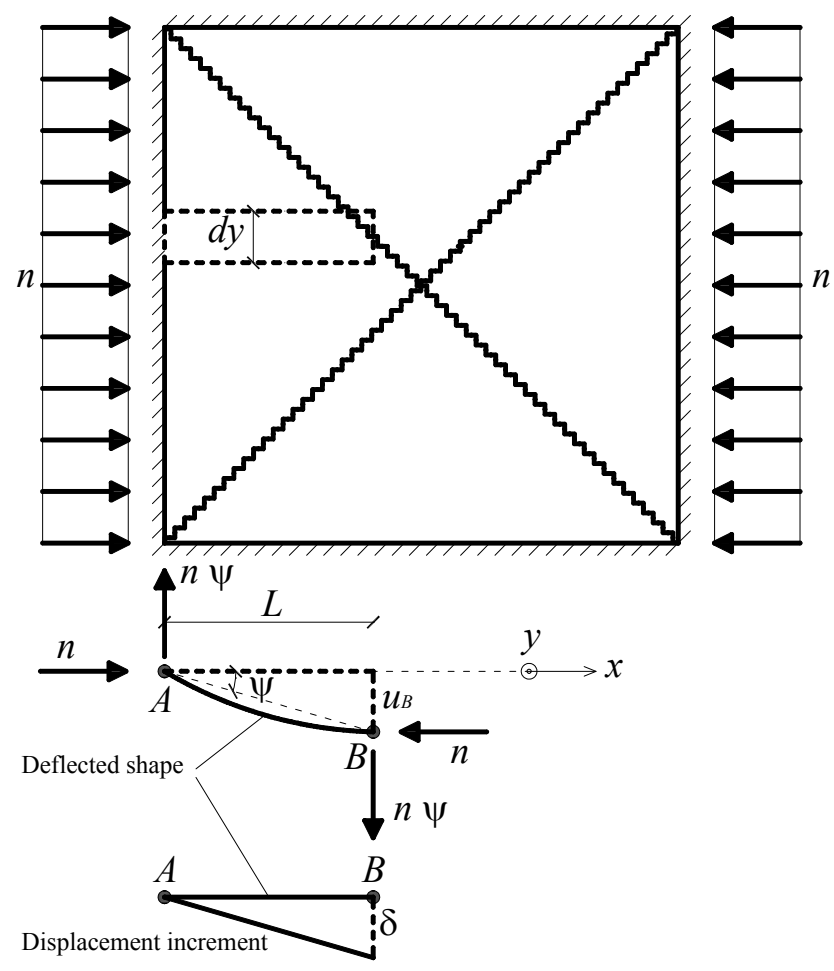

Figure 2. Plate Subdivided into Strips with Infinitesimal Widths 
The strip, marked by the dashed lines, has a normal force, $n$, per unit length. The transverse forces are a uniform load, $n \psi$, along the lines $(A$ and $B)$ acting upwards in $(A)$ (i.e. perpendicular to the $x, y$-plane), and a uniform load acting downwards in $(B)$. For a displacement increment, $\delta$, along the line $(B)$, the external work for the strip, $d_{y}$, is given by

$$
d W_{e}=n \psi d y \delta
$$

where the angular deflection increment, $\psi=u_{B} / L$. The dissipation contribution from the strip is determined by

$$
d W_{i}=m d y \frac{\delta}{L}
$$

where $m$ is calculated by Eq. 8 .

\subsection{Column Example}

The procedure is illustrated by a simply supported column, see Figure 3 . The deflected shape of the column is characterised by the deflection in the midpoint at maximum load, $u_{m}$. The column is centrally loaded by a compressive normal force, $N$, and thereby each half is subjected to the forces, $N \psi$. In the figure they are only shown in the midpoint.

The column is given a lateral displacement increment, $\delta$, at the midpoint, as shown in Figure 4, where a plastic yield hinge is formed.

The external work of the mechanism is

$$
W_{e}=2 N \psi \delta=4 N \frac{u_{m}}{L} \delta
$$

and the dissipation is

$$
W_{i}=4 \frac{M_{p}}{L} \delta
$$

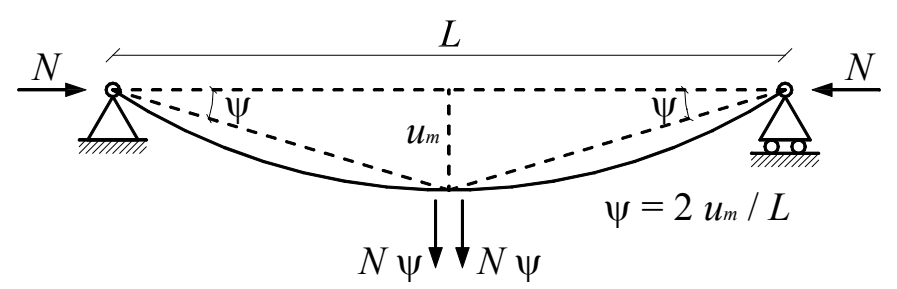

Figure 3. Deflected Shape of a Simply Supported Column

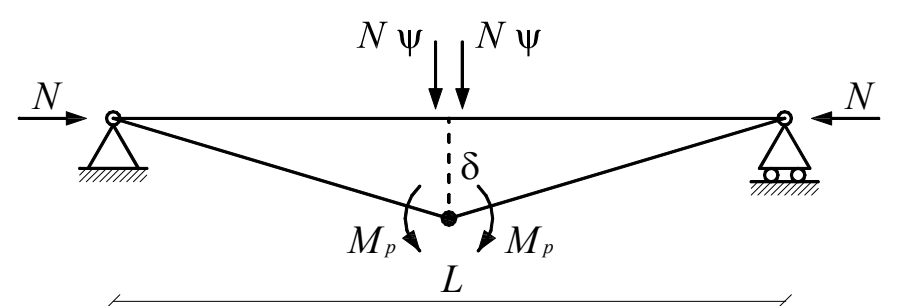

Figure 4. Failure Mechanism for a Lateral Displacement Increment, $\delta$ 
Here, $M_{p}$ is the plastic yield moment, which must be reduced due to the normal force.

The work equation renders:

$N u_{m}=M_{p}$

In this case just, the usual equilibrium equation is found.

A constant, solid and rectangular cross-section of the column is assumed in order to compare the result with the solutions derived later for plates. Hence, the following solution also corresponds to a rectangular plate with two free unloaded edges. The sides of the rectangular cross-section are denoted $b$ and $t$, respectively, where $b \geq t$ is assumed. The plastic yield moment, reduced due to the normal force, is then

$$
M_{p}=\frac{1}{4} b t^{2} f_{y}\left(1-\left(\frac{N}{N_{p}}\right)^{2}\right)
$$

$N$ is the normal force, $f_{y}$ the yield stress and $N_{p}=b t f_{y}$ is the load-carrying capacity in pure compression or tension.

The next step is to find a good estimate for the deflected shape of the column. The maximum deflection for a beam may in general be determined as

$u_{m}=\frac{1}{\alpha} \kappa L^{2}$

where $\kappa$ is the curvature in a selected point, $L$ the length and $\alpha$ is a parameter depending on the shape of the curvature function. In the following, $\alpha=8$, corresponding to a constant curvature function, is used.

In a beam or column with yielding, the deflection corresponding to maximum load tends to be reached when the yield strain, $\varepsilon_{y}$, is obtained in one or both faces. Here it is assumed that the deflection at maximum load may be found by assuming that the yield strain, $\varepsilon_{y}=f_{y} / E\left(f_{y}\right.$ is yield stress and $E$ Young's modulus), is reached in both faces. Thus the curvature is determined by $2 \varepsilon_{y} / t$. The resulting formula is modified by a parameter, $\mu$, in the following way:

$$
\kappa=\frac{2 \varepsilon_{y} \mu}{t}
$$

The parameter, $\mu$, is an empirical coefficient accounting for the effect of imperfections and residual stresses. Hence for the column in Figure 3, the deflection equals:

$u_{m}=\frac{1}{8} \frac{2 \varepsilon_{y} \mu}{t} L^{2}=\frac{1}{4} \mu \frac{f_{y}}{E} \frac{L^{2}}{t}$

Inserting Eq. 17 and Eq. 20 into Eq. 16, the load-carrying capacity expressed by the non-dimensional value, $N /\left(b t f_{y}\right)$, is found to be 
$\frac{N}{b t f_{y}}=-\frac{1}{2} \mu \lambda^{2}+\sqrt{\frac{1}{4} \mu^{2} \lambda^{4}+1}$

where the parameter, $\lambda$, has been introduced:

$\lambda=\frac{L}{t} \sqrt{\frac{f_{y}}{E}}$

Now this result is compared with the EC3 [4] formulae. Here a non-dimensional slenderness ratio, $\lambda_{r}$, given by

$\lambda_{r}=\sqrt{\frac{A f_{y}}{N_{c r}}}$

is introduced.

In this formula, $A$ is the cross-sectional area and $N_{c r}$ is the elastic critical buckling force (the Euler-load), see the following Eq. 27. For a solid, rectangular cross-section where $\lambda$ is given by Eq. $22, \lambda_{r}$ may be expressed as,

$\lambda_{r}=\frac{\sqrt{12}}{\pi} \sqrt{\frac{f_{y}}{E}} \frac{L}{t}=\frac{\sqrt{12}}{\pi} \lambda$

The load-carrying capacity according to EC3 [4] is given by

$\frac{N_{c r}}{b t f_{y}}=\frac{1}{\Phi+\sqrt{\Phi^{2}-\lambda_{r}^{2}}}$

where

$\Phi=0.5\left(1+\alpha\left(\lambda_{r}-0.2\right)+\lambda_{r}^{2}\right)$

The so-called geometric equivalent imperfection factor $\alpha$ in this equation may be obtained from Table 1. Notice that $\alpha$ here is not the same as the $\alpha$ introduced in Eq. 18.

The buckling curves according to EC3 [4], cf. Eq. 25, and the curve given by Eq. 21 for $\mu=1.4$ are shown in Figure 5. It turns out that by choosing a value of $\mu$ between 1.2 and 1.6, all buckling curves in EC3 [4] may be well represented. For $\mu=1.4$, the result coincides very closely with the buckling curve $b$. The figure also shows the Euler curve, which for a solid rectangular cross-section is given by

$\frac{N_{c r}}{b t f_{y}}=\frac{1}{\lambda_{r}^{2}}=\frac{\pi^{2}}{12} \lambda^{-2}$

Table 1. Imperfection Factors for Buckling Curves According to EC3

\begin{tabular}{|l|l|l|l|l|l|}
\hline Buckling curve & $a_{0}$ & $a$ & $b$ & $c$ & $d$ \\
\hline Imperfection factor $\alpha$ & 0.13 & 0.21 & 0.34 & 0.49 & 0.76 \\
\hline
\end{tabular}




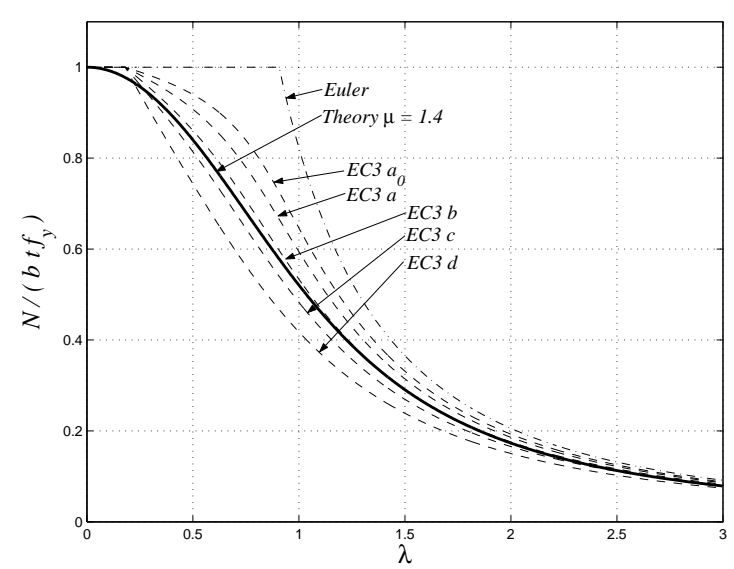

Figure 5. $N /\left(b t f_{y}\right)$ as a Function $\lambda$, Plastic Theory and EC3

If the column in Figure 3 is also subjected to a lateral load, $q$, per unit length along the entire length, $L$, then this load is easily included in the calculations. The work done by the lateral load is simply added to the external work, thus the work equation becomes, cf. Eq. 16,

$N u_{m}+\frac{1}{8} q L^{2}=M_{p}$

Other column cases may be treated in a similar way but this is not our purpose here.

The compression flange in a box-girder may be considered as a plate simply supported along all edges. How to determine the post-buckling strength of plates supported along all edges is presented below.

\subsection{Square Plates}

The square plate in Figure 6 is considered. It is simply supported along all four edges and subjected to a uniform load per unit length, $n$, along two opposite edges.

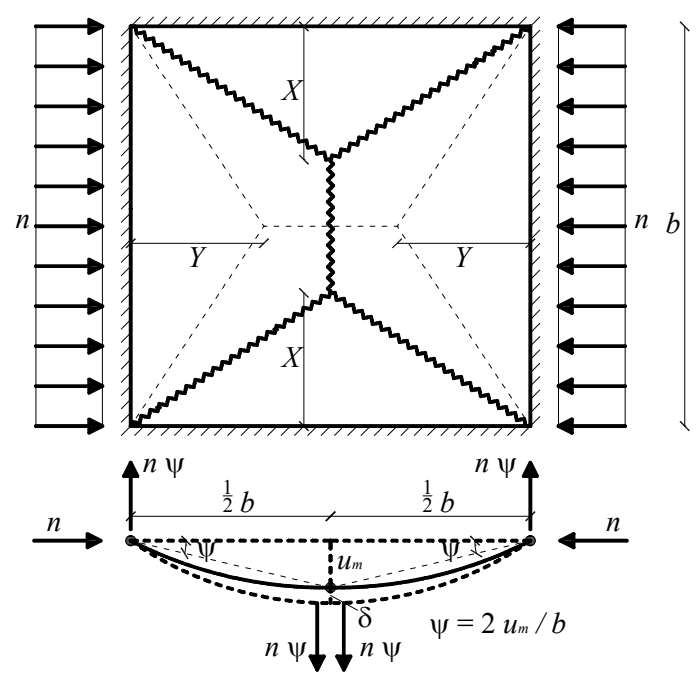

Figure 6. Failure Mechanism for a Simply Supported Square Plate 
The first step is to find a good estimate for the deflected shape of the plate. As stated in Section 2.3, the maximum deflection for a beam may in general be determined as

$u_{m}=\frac{1}{\alpha} \kappa L^{2}$

where $\kappa$ is the curvature, $L$ the length and $\alpha$ is a parameter depending on the shape of the curvature function. In the following $\alpha=8$ is used, corresponding to a constant curvature function.

In a plate with yielding, the deflection corresponding to maximum load, as mentioned above, tends to be reached when the yield strain, $\varepsilon_{y}$, is obtained in one or both faces. As in the case of a column, cf. Section 2.3, the deflection at maximum load may be found by assuming that the yield strain, $\varepsilon_{y}=f_{y} / E$, is reached in both faces of the plate. Here, $f_{y}$ is the yield stress and $E$ is the Young's modulus. In this case $\mu=1$ is applied, cf. Eq. 19, thus the curvature is determined by ( $t$ being the plate thickness)

$\kappa=\frac{2 \varepsilon_{y}}{t}$

For a square plate, $L$ is taken equal to the side length, $b$. Hence, for the plate in Figure 6 , the deflection at maximum load, $u_{m}$, is

$u_{m}=\frac{1}{8} \frac{2 \varepsilon_{y}}{t} b^{2}=\frac{1}{4} \frac{f_{y}}{E} \frac{b^{2}}{t}$

The next step is to find the optimal failure mechanism (yield line pattern). In Figure 6, two different yield line patterns are shown, one with the free parameter, $X$, and one with the free parameter, $Y$ (shown with dashed lines), respectively.

For the mechanism with the free parameter, $X$, the external work for a displacement increment, $\delta$, equals

$$
\begin{aligned}
& W_{e}=4 X n \frac{2 u_{m}}{b} \frac{\delta}{2}+2(b-2 X) n \frac{2 u_{m}}{b} \delta \\
& W_{e}=n u_{m} \delta\left(4-4 \frac{X}{b}\right)
\end{aligned}
$$

and the dissipation is

$$
W_{i}=4 m_{p} \delta\left(1-\left(\frac{n}{n_{p}}\right)^{2}\right)+2 m_{p} \delta \frac{b}{X}
$$

Here, the plastic yield moment, $m_{p}$, per unit length is given by Eq. 7 and $n_{p}=t f_{y}$ is the load-carrying capacity in pure compression or tension.

Equalising the external work and the dissipation and inserting Eq. 7 and Eq. 31, the load-carrying capacity, expressed by the non-dimensional parameter, $n /\left(t f_{y}\right)$, may be written as 
$\frac{n}{t f_{y}}=-\frac{1}{2} \lambda^{2}\left(1-\frac{X}{b}\right)+\sqrt{\frac{1}{4} \lambda^{4}\left(1-\frac{X}{b}\right)^{2}+\frac{1}{2}\left(2+\frac{b}{X}\right)}$

where the parameter, $\lambda$,

$\lambda=\frac{b}{t} \sqrt{\frac{f_{y}}{E}}$

has been introduced.

Eq. 34 is the post-buckling load in this case. The value of $n /\left(t f_{y}\right)$ as a function of $\lambda$ is shown for different values of $X$ in Figure 7.

It is seen that $n /\left(t f_{y}\right)>1$ for small values of $\lambda$, which is not possible in reality, hence a cut-off at $n /\left(t f_{y}\right)=1$ must be introduced. With this cut-off, it appears that almost the same load-carrying capacity is obtained for $X=1 / 2 b, X=2 / 5 b$ and $X=1 / 3 b$.

The mechanism with the free parameter, $Y$, cf. Figure 6 , leads to $Y=0$ when optimised. However, it must be remembered that the deflected shape is used both as an estimate of the deflection at maximum load and as the basis for a choice of the deflection increment at the yield load. Thus a normal optimisation may not be appropriate. The optimised value for $Y=0$ is therefore disregarded and the mechanism corresponding to $X=1 / 2 b(Y=1 / 2 b)$ is chosen for the following calculations.

In Figure 8 , the ratio, $n /\left(t f_{y}\right)$, as a function of $\lambda$ for $X=1 / 2 b$ is shown together with the elastic solution, which may be written as, cf. Eq. 1,

$\frac{n}{t f_{y}}=\frac{\pi^{2} k}{12\left(1-v^{2}\right)} \lambda^{-2}$

where $k$ is the buckling coefficient and $v$ is Poisson's ratio. Here $k=4$ and $v=0.30$ are used. The parameter, $\lambda$, is given by Eq. 35 .

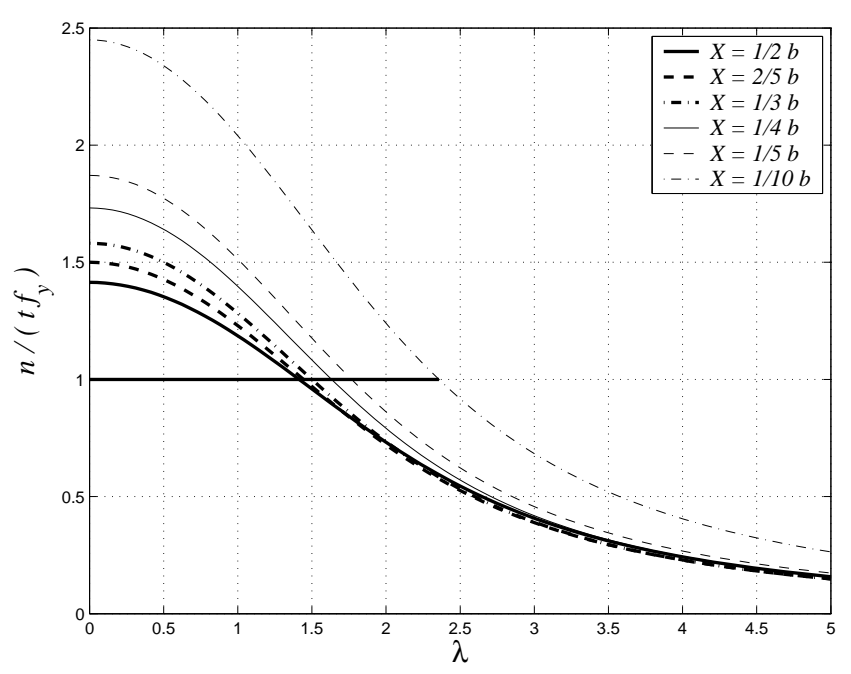

Figure 7. $n /\left(t f_{y}\right)$ as a Function of $\lambda$ for Different Values of $X$ 
The two curves coincide very closely, which would indicate that no extra post-buckling reserve is found from this approach. However, the result is incorrect, which is due to the fact that a redistribution of the stresses occurs when the buckling mechanism develops. This is illustrated in Figure 9.

As previously mentioned, Kármán et al. [8] suggested that the redistribution may be taken into account by using an effective area subjected to a uniform stress equal to the yield stress, i.e. $\sigma=f_{y}$ in Figure 9. The same simplification will be used here. Hence the considered plate is subjected to a load per unit length equal to the yield stress multiplied by the thickness of the plate, i.e. $n=n_{p}=t f_{y}$, which is applied along two strips with the width equal to the unknown width $b_{s}$, see Figure 10.

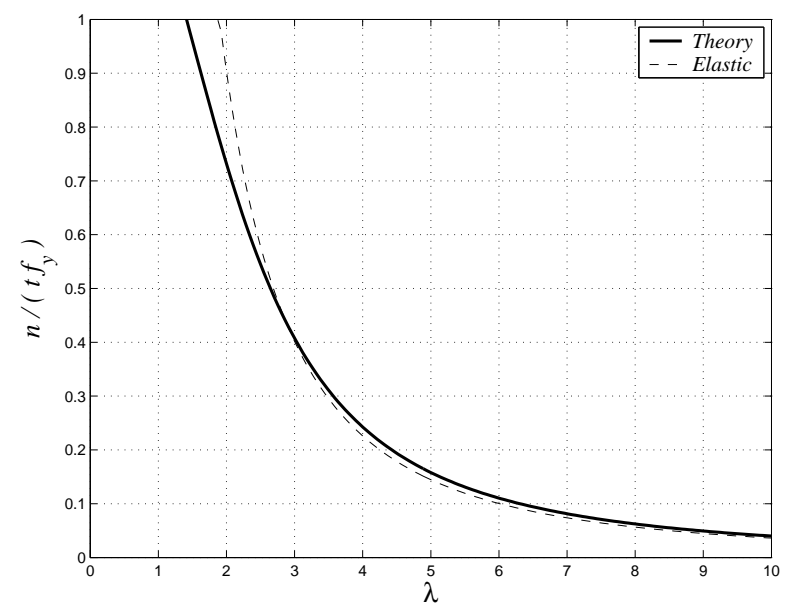

Figure 8. $n /\left(t f_{y}\right)$ as a Function of $\lambda$ for $X=1 / 2 b$

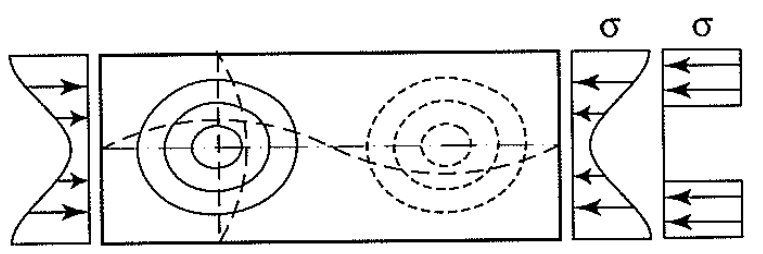

Figure 9. Redistribution of Edge Stresses During Buckling

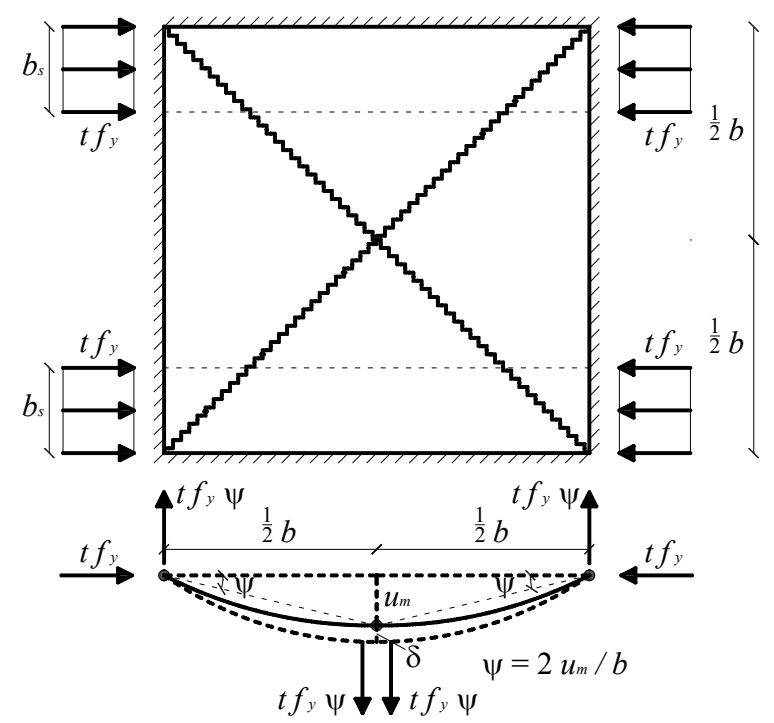

Figure 10. Simply Supported Square Plate Subjected to Uniaxial Compression Along Strips of width $b_{s}$ 
The external work, $W_{e}$, and the dissipation, $W_{i}$, are for $\delta=1$, respectively:

$$
\begin{aligned}
& W_{e}=8 t f_{y} u_{m} \frac{b_{s}^{2}}{b^{2}} \\
& W_{i}=\underbrace{\left.8 m_{p} \frac{\left.b_{s}\left(\frac{t f_{y}}{b}\right)^{2}\right)}{n_{p}}\right)^{1-\left(\frac{b_{s}}{b}\right)}+8 m_{p}\left(1-\frac{b_{s}}{b}\right)}_{=0}
\end{aligned}
$$

Again, $u_{m}$ is the deflection at maximum load, $m_{p}$ is the plastic yield moment per unit length given by Eq. 7 and $n_{p}=t f_{y}$ is the load-carrying capacity in pure compression or tension.

The contribution from the part of the yield lines running in the widths, $b_{s}$, is equal to zero, as $n_{p}=t f_{y}$. Inserting Eq. 7 and Eq. 31, and using $W_{i}=W_{e}, b_{s}$ is found to be determined by

$$
\frac{f_{y}}{E} b_{s}^{2}+\frac{t^{2}}{b} b_{s}-t^{2}=0
$$

Denoting the total effective width $b_{e}=2 b_{s}$, we find

$$
\frac{b_{e}}{b}=-\lambda^{-2}+\sqrt{\lambda^{-4}+4 \lambda^{-2}}
$$

where $\lambda$ is given by Eq. 35 .

The average stress relative to the yield stress, $f_{y}$, along the whole width, $b$, from the yield force, $b_{e} t$ $f_{y}$, along $b_{e}$ is $b_{e} t f_{y} /\left(b t f_{y}\right)=b_{e} / b$. For a solution with uniform load, $n$, along the whole width, $b$, the average stress relative to the yield stress is $n /\left(t f_{y}\right)$.

In Figure $11, b_{e} / b$, cf. Eq. 40 , is shown as a function of $\lambda$.

The elastic buckling load characterised by $n /\left(t f_{y}\right)$, cf. Eq. 36 , is also shown. It appears that the plate may carry compressive forces considerably above the elastic buckling load. The first to demonstrate this through tests appear to be Schuman and Back [12].

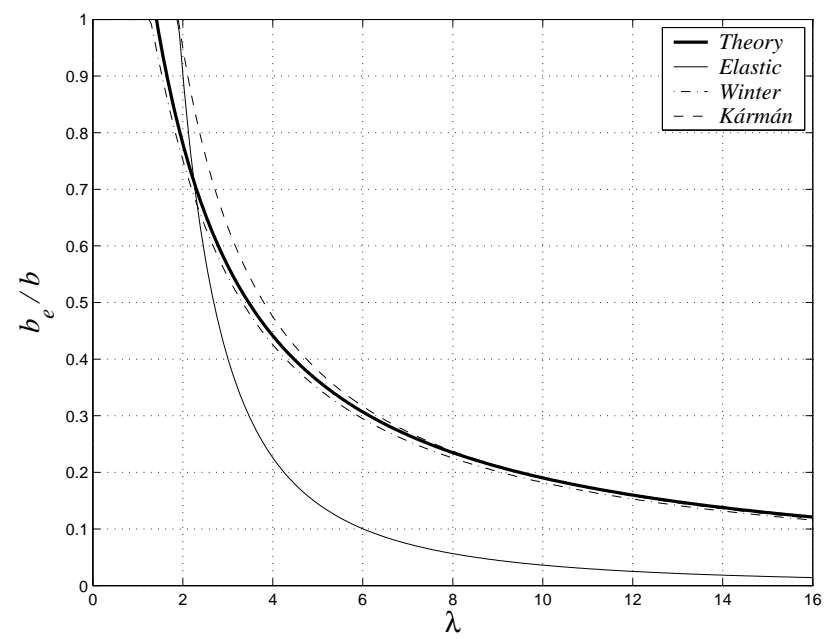

Figure $11 . b_{e} / b$ as a function of $\lambda$ 
The third curve plotted in the figure is the semi-empirical expression derived by Winter [15]. With the introduced parameter, $\lambda$, given by Eq. 35 , it may be written as, cf. Eq. 4 ,

$$
\frac{b_{e}}{b}=\frac{\pi \sqrt{k}}{\sqrt{12\left(1-v^{2}\right)}} \lambda^{-1}\left(1-0.22 \frac{\pi \sqrt{k}}{\sqrt{12\left(1-v^{2}\right)}} \lambda^{-1}\right)
$$

Finally, a cut-off at $b_{e} / b=n /\left(t f_{y}\right)=1$ has been introduced.

Winter's formula is a modification of the original formula introduced by Kármán et al. [8]:

$$
\frac{b_{e}}{b}=C \frac{t}{b} \sqrt{\frac{E}{f_{y}}}=C \lambda^{-1}
$$

where $C$ is an empirical constant. Based on the tests made by Schuman and Back [12], $C=1.9$ was found. Eq. 42 is also shown in the figure.

Regarding Eq. 42, Winter [15] argued that $C$ should depend on the parameter $\lambda^{-1}$. Based on his own tests and those made by Sechler (Winter [15]), he found the best fit to be

$$
C=1.9-1.09 \frac{t}{b} \sqrt{\frac{E}{f_{y}}}=1.9-1.09 \lambda^{-1}
$$

As previously mentioned, this formula has later been modified several times.

Eq. 41 is the newest modification and it is adopted in EC3 [4]. Eq. 41 corresponds, for $k=4$ and for $v=0.30$, to

$$
C=1.9-0.42 \lambda^{-1}
$$

Figure 11 shows that the present theoretical result closely follows Winter's semi-empirical solution.

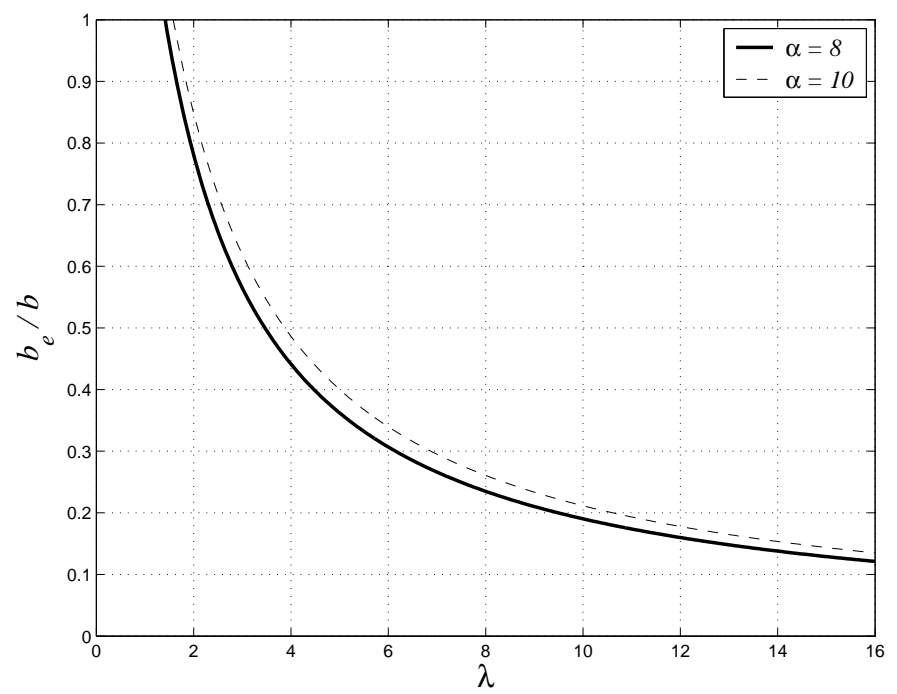

Figure 12. $b_{e} / b$ as a Function of $\lambda$ for $\alpha=8$ and $\alpha=10$, respectively 
In order to investigate the sensitiveness of the solution, Eq. 40, to the estimated deflection at maximum load, it is compared with the solution using $\alpha=10$ in Figure 12.

Choosing $\alpha=10$ in Eq. 29 approximately corresponds to a sinusoidal curvature function $\left(\alpha=\pi^{2} \approx\right.$ $10)$. For $\alpha=10$, the effective width is determined by

$$
\frac{b_{e}}{b}=-\frac{5}{4} \lambda^{-2}+\sqrt{\frac{25}{16} \lambda^{-4}+5 \lambda^{-2}}
$$

where $\lambda$ is still given by Eq. 35 .

Eq. 45 gives a post-buckling strength up to $11 \%$ larger than that obtained by Eq. 40 for the $\lambda$-interval shown in Figure 12. Hence, the theory seems to be relatively insensitive to the estimated deflection at maximum load.

When von Mises' yield criterion is applied, $m_{p}$ in Eq. 38 is replaced by $2 m_{p} / \sqrt{ } 3$ if uniaxial strain is supposed. In that case also $n_{p}$ should be increased and $b_{e} / b$ then will measure $n$ relative to the increased value of $n_{p}$. Thus it turns out that nothing is changed, since $b_{e} / b$ is still given by, cf. Eq. 40 ,

$$
\frac{b_{e}}{b}=-\lambda^{-2}+\sqrt{\lambda^{-4}+4 \lambda^{-2}}
$$

In practise one is on the safe side by using the uniaxial yield stress. Thus the former solution is applied.

\subsection{Rectangular Plates}

It is a well-known fact that a long rectangular elastic plate subjected to compression in the longitudinal direction buckles into a shape of half waves with a length equal to the plate width, see Figure 13. This result is applied when estimating the post-buckling strength. Thus a long plate is subdivided into a number of square plates where the previous yield line patterns may be applied, cf. Section 3.1. The vertical lines between the square regions will act as simple supports, since if one square region forms a wave downwards, then the adjacent regions will form a wave upwards. Therefore, the post-buckling strength or the effective width for a long rectangular plate is in general given by Eq. 40.

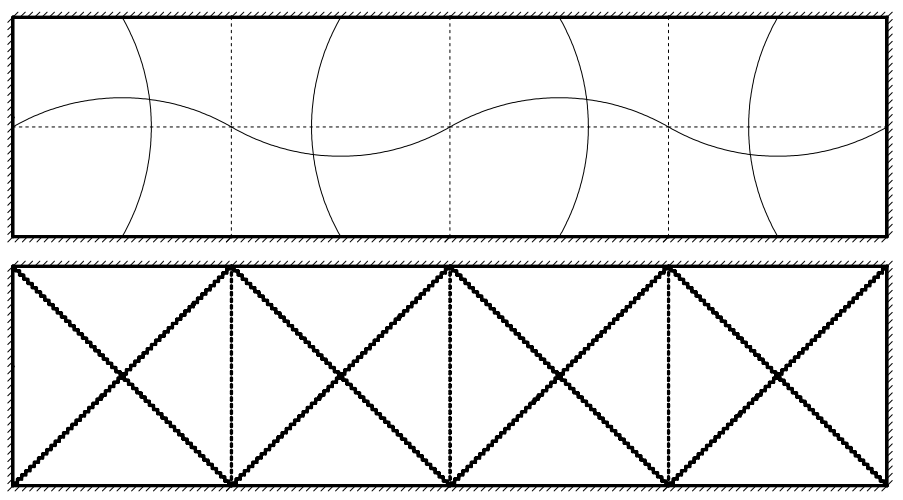

Figure 13. Deflection Shape and Failure Mechanism for Long Simply Supported Rectangular Plates 
Now consider a rectangular plate with a length somewhat larger than the width. In Figure 14, the width is named $b$ and the length named $a$. The plate is subjected to uniaxial compression, $n_{p}=t f_{y}$, acting on the two strips, $b_{s}$.

The failure mechanism has the free parameter, $X$. The maximum deflection, $u_{m}$, is assumed to be given by Eq. 31. The external work is then for $\delta=1$ given by

$$
W_{e}=4 t f_{y} \frac{u_{m}}{X} \frac{b_{s}^{2}}{b}
$$

and the dissipation

$$
W_{i}=2 m_{p}\left(\frac{b}{X}-2 \frac{b_{s}}{X}+2 \frac{a}{b}\right)
$$

Hence, $b_{s}$ may be determined by

$$
\frac{f_{y}}{E} b_{s}^{2}+\frac{t^{2}}{b} b_{s}-t^{2}\left(\frac{1}{2}+\frac{X a}{b^{2}}\right)=0
$$

The effective width, $b_{e}\left(b_{e}=2 b_{s}\right)$, is then found to be

$$
\frac{b_{e}}{b}=-\lambda^{-2}+\sqrt{\lambda^{-4}+4\left(\frac{1}{2}+\frac{X a}{b^{2}}\right) \lambda^{-2}}
$$

where $\lambda$ is given by Eq. 35. Furthermore, in the above equations, $t$ is the thickness, $E$ is Young's modulus, $f_{y}$ the yield stress, $m_{p}$ is the plastic yield moment per unit length given by Eq. 7, and $n_{p}=t f_{y}$ is the load-carrying capacity in pure compression or tension.

Assuming $X=1 / 2 b$, the effective width is determined by

$$
\frac{b_{e}}{b}=-\lambda^{-2}+\sqrt{\lambda^{-4}+2\left(1+\frac{a}{b}\right) \lambda^{-2}}
$$

When $a$ is larger than, or equal to, $b$, it appears that Eq. 51 gives the smallest post-buckling strength if $a$ is equal to $b$. Hence in practice, the yield line pattern for a square plate may be considered as the optimal solution, rather than the pattern shown in Figure 14.

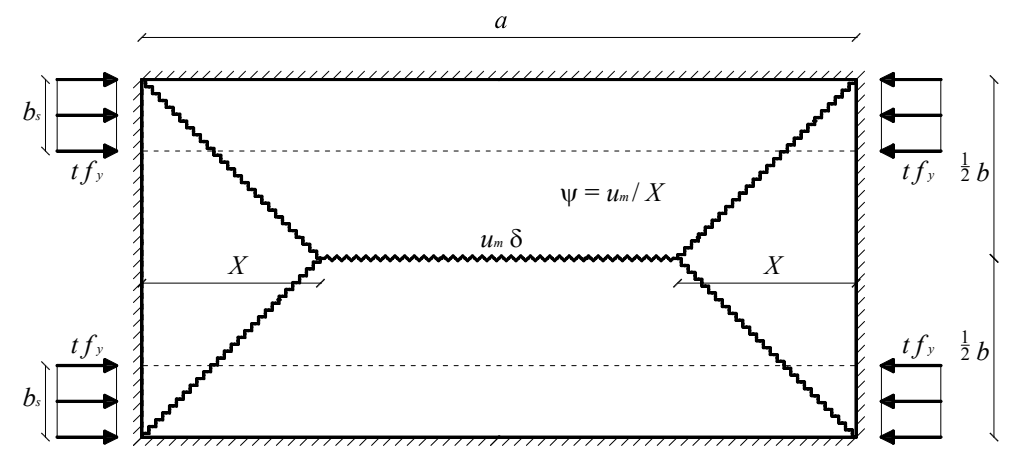

Figure 14. Failure Mechanism for a Short Rectangular Plate 
Special attention is required for plates where $a$ is smaller than $b$, but the theory may also easily be applied to this case.

The yield line pattern shown in Figure 15 is considered, where $a$ is still larger than $b$ but the loaded strips are now vertical. The plate is subjected to a load per unit length equal to the yield stress, $f_{y}$, multiplied by the thickness, $t$, along two strips of width $b_{s}$. The failure mechanism has the free parameter, $X$. The work equation must be derived separately for the two cases, $b_{s}<X$ and $b_{s}>X$, respectively.

In the case $b_{s}<X$, the external work for $\delta=1$ is given by

$W_{e}=4 t f_{y} \frac{u_{m}}{b X} b_{s}^{2}$

and the dissipation

$W_{i}=4 m_{p}\left(\frac{a}{b}-2 \frac{b_{s}}{b}+\frac{1}{2} \frac{b}{X}\right)$

where $m_{p}$ is the plastic yield moment per unit length given by Eq. 7 .

The maximum deflection, $u_{m}$, is taken as the deflection of a beam in the direction of the uniaxial compression with the curvature given by Eq. 30 . Thus

$u_{m}=\frac{1}{8} \frac{2 \varepsilon_{y}}{t} b^{2}=\frac{1}{4} \frac{f_{y}}{E} \frac{b^{2}}{t}$

Equalising the external work and the dissipation, the width of each strip, $b_{s}$, for $b_{s}<X$ is found to be determined by

$\frac{f_{y}}{E} b_{s}^{2}+2 \frac{t^{2} X}{b^{2}} b_{s}-t^{2}\left(\frac{a X}{b^{2}}+\frac{1}{2}\right)=0$

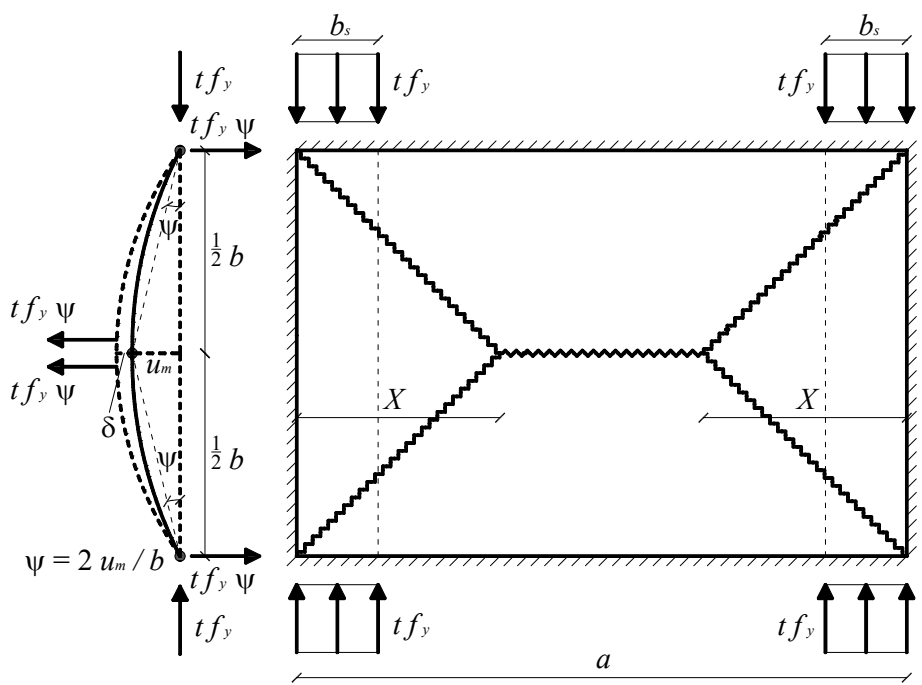

Figure 15. Failure Mechanism for a Web Plate with $a>b$ and $b_{s}<X$ 
In the case $b_{s}>X$, the external work for $\delta=1$ is given by

$$
W_{e}=4 t f_{y} \frac{u_{m}}{b}\left(2 b_{s}-X\right)
$$

and the dissipation, which is identical to Eq. 53, is

$$
W_{i}=4 m_{p}\left(\frac{a}{b}-2 \frac{b_{s}}{b}+\frac{1}{2} \frac{b}{X}\right)
$$

Equalising the external work and the dissipation, the width of each strip, $b_{s}$, for $b_{s}>X$ is found to be determined by

$$
\left(2 \frac{f_{y}}{E} b+2 \frac{t^{2}}{b}\right) b_{s}=\frac{f_{y}}{E} b X+t^{2}\left(\frac{a}{b}+\frac{1}{2} \frac{b}{X}\right)
$$

In Section 3.1, it was shown that inclined yield lines under an angle of $45^{\circ}$ are normally a good choice, noting that minimising $b_{s}$ with regard to $X$ did not lead to any useful result. Hence, an angle of $45^{\circ}$ is also chosen here, i.e. $X=1 / 2 b$.

Denoting the effective width $b_{e}=2 b_{s}$, the effective width for $b_{s}<X\left(\Rightarrow b_{e}<b\right)$ is given by

$$
\frac{b_{e}}{a}=-\lambda^{-2} \frac{b}{a}+\sqrt{\lambda^{-4}\left(\frac{b}{a}\right)^{2}+2 \lambda^{-2}\left(\frac{b^{2}}{a^{2}}+\frac{b}{a}\right)}
$$

and for $b_{s}>X\left(\Rightarrow b_{e}>b\right)$

$$
\frac{b_{e}}{a}=\frac{\frac{1}{2} \lambda^{2} \frac{b}{a}+\frac{b}{a}+1}{\lambda^{2}+1}
$$

In both Eq. 59 and Eq. $60, b_{e} / b \leq 1$ is required, and $\lambda$ is given by Eq. 35 .

The parameter, $b_{e} / a$, through Eq. 59 and Eq. 60 as a function of $\lambda$ is shown for different values of $a / b$ in Figure 16. In Figure 17, the ratio, $b_{e} / b$, as a function of $\lambda$ is shown for different values of $a / b$. For the two cases treated above, the ratio, $b_{e} / b$, may be written as:

For $b_{e} / b \leq 1$ :

$$
\frac{b_{e}}{b}=-\lambda^{-2}+\sqrt{\lambda^{-4}+2 \lambda^{-2}\left(\frac{a}{b}+1\right)}
$$

For $b_{e} / b \geq 1$ :

$$
\frac{b_{e}}{b}=\frac{\frac{1}{2} \lambda^{2}+\frac{a}{b}+1}{\lambda^{2}+1}
$$


By inserting $b_{e} / b=1$ in either Eq. 61 or Eq. 62 , it is found that the two curves, for any value of $a / b$, in both Figure 16 and Figure 17, intersect for

$$
\lambda=\sqrt{2 \frac{a}{b}}
$$

Since, $b_{e} \leq a$ is required, a cut-off at $b_{e} / a=1$, as shown in Figure 16, must be done for all curves. In Figure 17, it is shown that each curve has a cut-off for $b_{e} / b$ equal to the value of $a / b$ corresponding to the actual curve. By inserting $b_{e}=a$ into, for instance, Eq. 62, it is verified that the cut-off, in both Figure 16 and Figure 17, takes place for

$$
\lambda=\left(\frac{a}{b}-\frac{1}{2}\right)^{-1 / 2}
$$

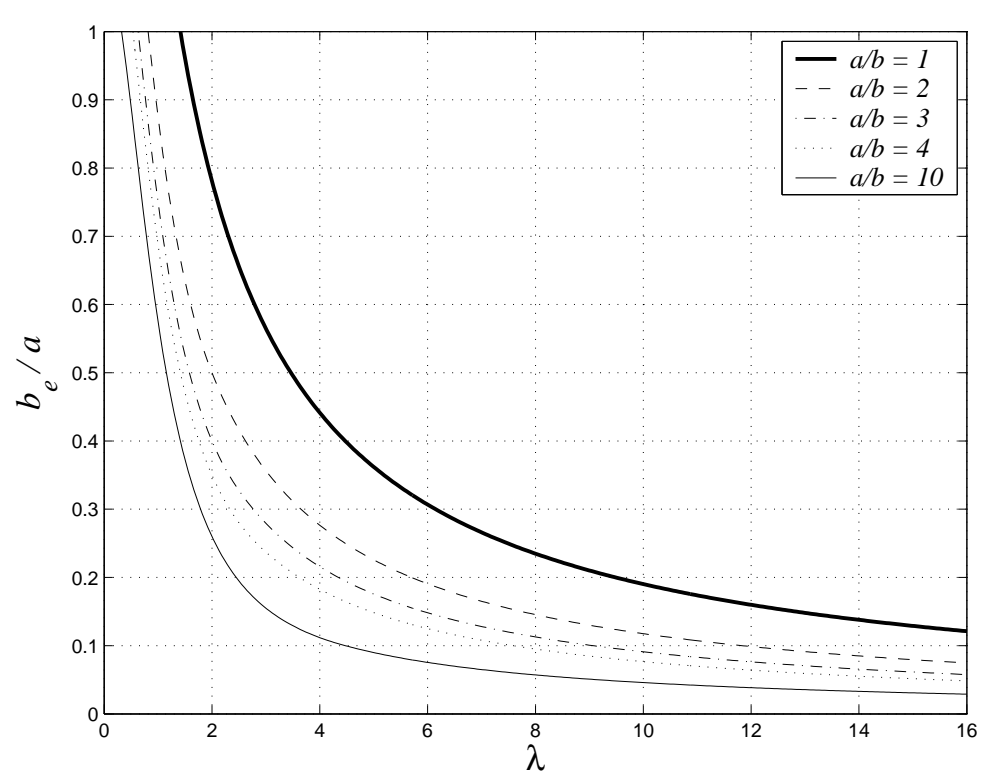

Figure 16. $b_{e} / a$ as a Function of $\lambda$ for Different Values of $a / b$

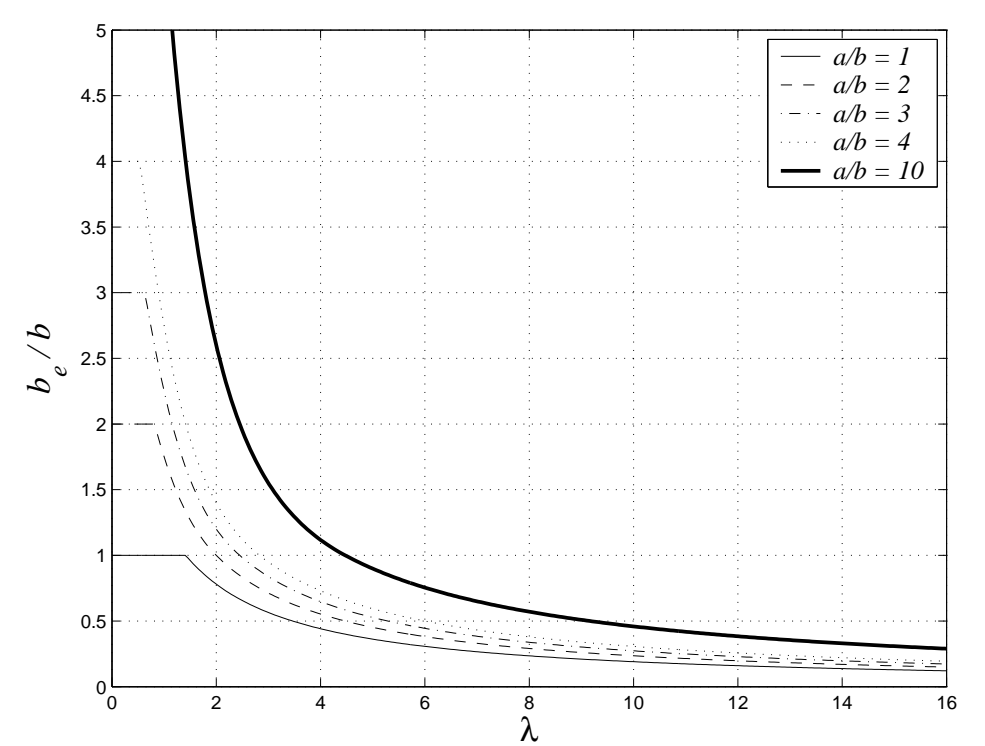

Figure 17. $b_{e} / b$ as a Function of $\lambda$ for Different Values of $a / b$ 


\subsection{Comparison with Experimental Results}

The Schuman and Back [12] tests dealt with separate rectangular flat plates of four different metals, i.e. Duralumin, Stainless Iron, Monel Metal and Nickel. Eq. 40 is compared to these tests in Figure 18. The figure also shows the elastic solution, cf. Eq. 36, Winter's solution cf. Eq. 41 and the solution by Kármán et al. cf. Eq. 42. From the figure it appears that all formulae, except the elastic solution, overestimate the post-buckling strength, in some cases considerably. However, it is generally accepted that these experiments are unreliable because of the dubious V-groove supports. Hence, these experiments are not treated further here.

In Figure 19, the theories are compared with more reliable experiments.

The U-beams and I-beams tests made by Winter [15] both consisted of specimens made by bolting or welding U-sections together. Winter also used the tests made by Sechler (Winter [15]) to verify his method. The specimens in these tests were single plates, unconnected to any adjacent elements. These tests have been shown in Figure 19. Furthermore, newer tests by Moxham [9] are included in the figure. He conducted three test series, denoted Welded, Unwelded and Short in the following. Also, in these tests, all specimens were separate plates. He developed a new test rig, where he could establish the simple support conditions in a reliable way. In the Welded series, the longitudinal edges were heat treated in order to induce residual stresses. The Short series was conducted on specimens where the loaded edges were slightly longer than the unloaded edges (length-to-width ratio: 0.875 ). The theoretical effective width of the short specimens is calculated by Eq. 59 and Eq. 60. The specimens in the Welded and Unwelded series all had a length-to-width ratio of 4.0.

The agreement with all the tests seems to be very good. In Figure 20, the correlation between the present theory and tests is shown in a more illustrative way. For all tests, a mean value of 1.088 and a standard deviation of $16.9 \%$ are obtained. For the separate test series, the following results are obtained:

- Sechler: Mean 1.202, standard deviation $17.9 \%$.

- Winter, U-beams: Mean 1.098, standard deviation $18.9 \%$.

- Winter, I-beams: Mean 1.019, standard deviation $4.6 \%$.

- Moxham, Welded: Mean 1.097, standard deviation 12.2\%.

- Moxham, Unwelded: Mean 0.931, standard deviation $5.8 \%$.

- Moxham, Short: Mean 0.946, standard deviation $6.4 \%$.

It may be seen that the tests by Sechler deviate somewhat from the theory, especially for $b_{e} / b$ close to unity. One explanation for this might be initial imperfections, see Chapter 5. Moreover, he may have applied the same doubtful V-groove supports as Schuman and Back [12]. Without Sechler's tests, a mean value of 1.045 and a standard deviation of $14.4 \%$ are obtained. 


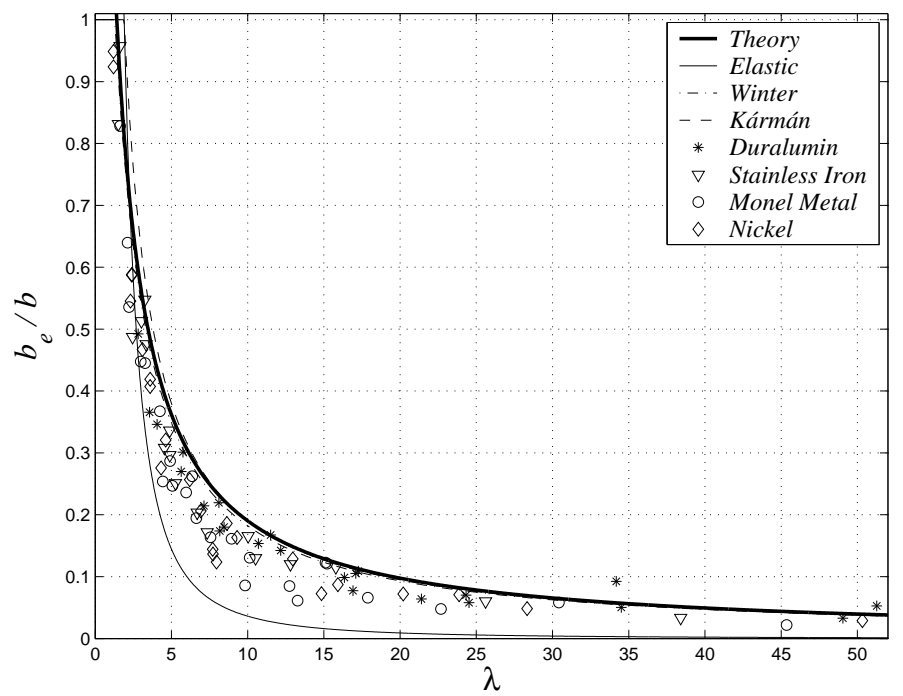

Figure 18. $b_{e} / b$ as a Function of $\lambda$, Theories Compared with Tests by Schuman and Back

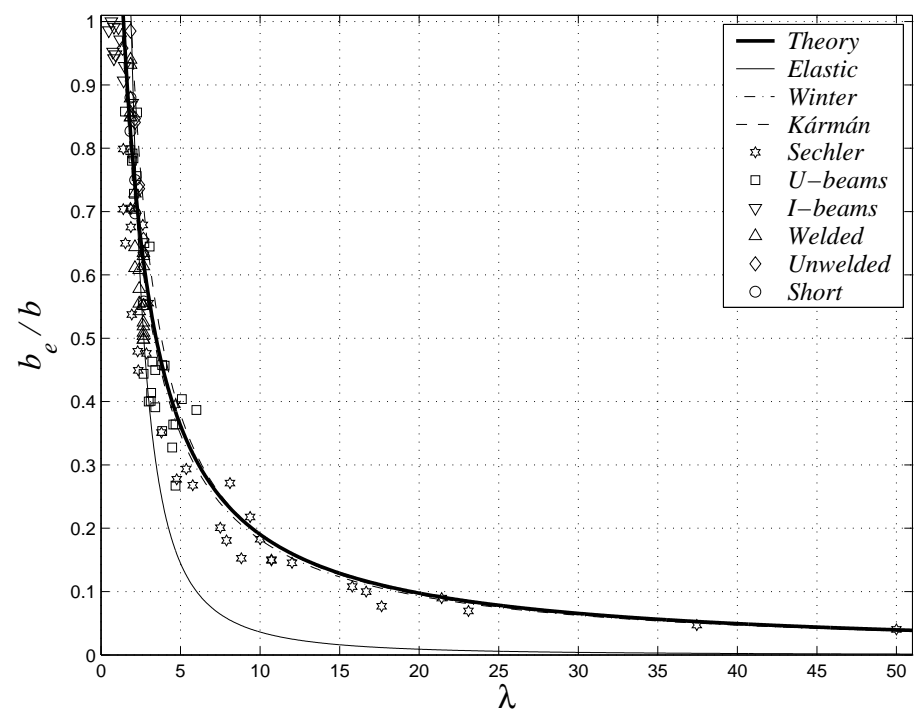

Figure 19. $b_{e} / b$ as a Function of $\lambda$, Theories and Tests

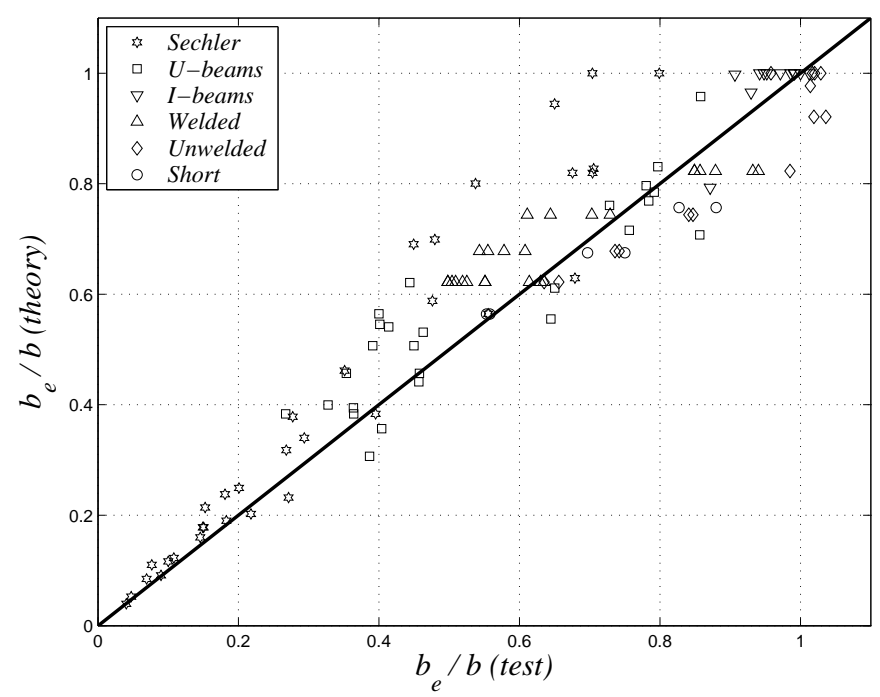

Figure 20. Theory Versus Tests 


\section{PLATES WITH ONE FREE EDGE}

The compressive flange and the transverse web stiffeners in an I-shaped steel plate girder may be considered to be long, rectangular plates simply supported along three of the edges, and free along one longitudinal edge. How to determine the post-buckling strength of such plates is presented below.

\subsection{Rectangular Plates}

The considered plate with length $a$, width $b$ and thickness $t$ is subjected to uniaxial compression, $n_{p}=t f_{y}$, along an effective width, $b_{e}$, near the supported longitudinal edge.

The best failure mechanism, with free parameter, $X$, is shown in Figure 21. It is easily verifiable that plates with length, $a$, in the interval, $2 X \leq a \leq \infty$, will have the same post-buckling strength. Plates with $a<2 X$ require another failure mechanism, which will be treated in Section 4.3.

The two regions with area, $X b$, will approximately be subjected to pure torsion. Thus the principal directions are under $45^{\circ}$ with the edges, and the principal curvatures are equal, but with opposite sign. In the principal directions, the curvatures are estimated to be, in absolute value, the same as in Eq. 30. Then the torsional curvature, $\kappa_{x y}$, will also be $\kappa_{x y}=2 \varepsilon_{y} / t\left(\varepsilon_{y}=f_{y} / E\right.$ is the yield strain and $t$ the thickness), which means that the deflection, $u_{m}$, at the end point of the yield line in the free edge will be

$u_{m}=\kappa_{x y} X b=\frac{2 \varepsilon_{y}}{t} X b=2 \frac{f_{y}}{E} \frac{X b}{t}$

For the mechanism shown in Figure 21, the external work is for $\delta=1$ given by

$W_{e}=t f_{y} \frac{u_{m}}{X} \frac{b_{e}^{2}}{b}$

and the dissipation is

$W_{i}=2 m_{p}\left(\frac{X}{b}+\frac{b}{X}-\frac{b_{e}}{X}\right)$

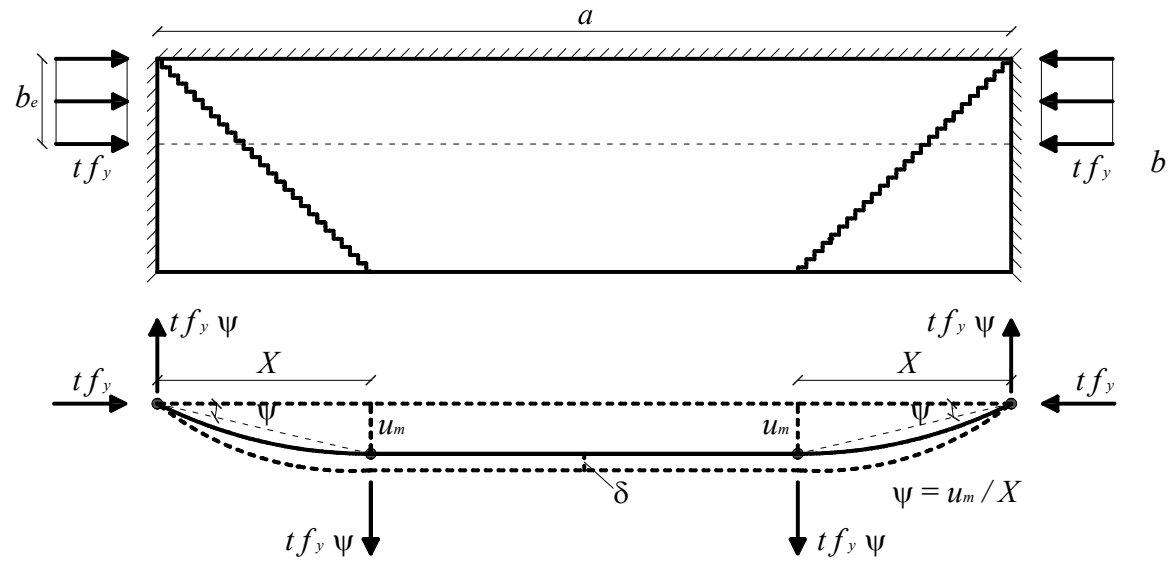

Figure 21. Failure Mechanism for a Rectangular Plate with One Free Edge 
where $m_{p}$ is given by Eq. 7. Hence the effective width, $b_{e}$, may be determined by

$2 \frac{f_{y}}{E} b_{e}^{2}+\frac{1}{2} \frac{t^{2}}{X} b_{e}-\frac{1}{2} t^{2}\left(\frac{X}{b}+\frac{b}{X}\right)=0$

The non-dimensional value, $b_{e} / b$, is then given by

$$
\frac{b_{e}}{b}=-\frac{1}{8} \frac{b}{X} \lambda^{-2}+\sqrt{\frac{1}{64}\left(\frac{b}{X}\right)^{2} \lambda^{-4}+\frac{1}{4}\left(\frac{X}{b}+\frac{b}{X}\right) \lambda^{-2}}
$$

Eq. 69 is shown for different values of $X$ in Figure 22. A cut-off at $b_{e} / b=1$ must again be introduced. With this cut-off, it is seen that almost the same load-carrying capacity is obtained for $X=b, X=4 / 5 b$ and $X=3 / 4 b$; hence $X=b$ is used in the following calculations.

The effective width for $X$ equal to $b$ is found to be

$$
\frac{b_{e}}{b}=-\frac{1}{8} \lambda^{-2}+\sqrt{\frac{1}{64} \lambda^{-4}+\frac{1}{2} \lambda^{-2}}
$$

which is shown in Figure 23. The figure also shows the elastic buckling curve given by Eq. 36 with $k=0.43$, cf. (Timoshenko and Gere [14]). Again, Poisson's ratio $v=0.30$ is assumed. The third curve shown is Winter's formula in the form given in EC3 [4], which may be written as, cf. Eq. 6 ,

$$
\frac{b_{e}}{b}=\frac{\pi \sqrt{k}}{\sqrt{12\left(1-v^{2}\right)}} \lambda^{-1}\left(1-0.188 \frac{\pi \sqrt{k}}{\sqrt{12\left(1-v^{2}\right)}} \lambda^{-1}\right)
$$

Here, $k=0.43$ and $v=0.30$ are again used. It is found that the theory gives a slightly larger post-buckling strength than Winter's solution.

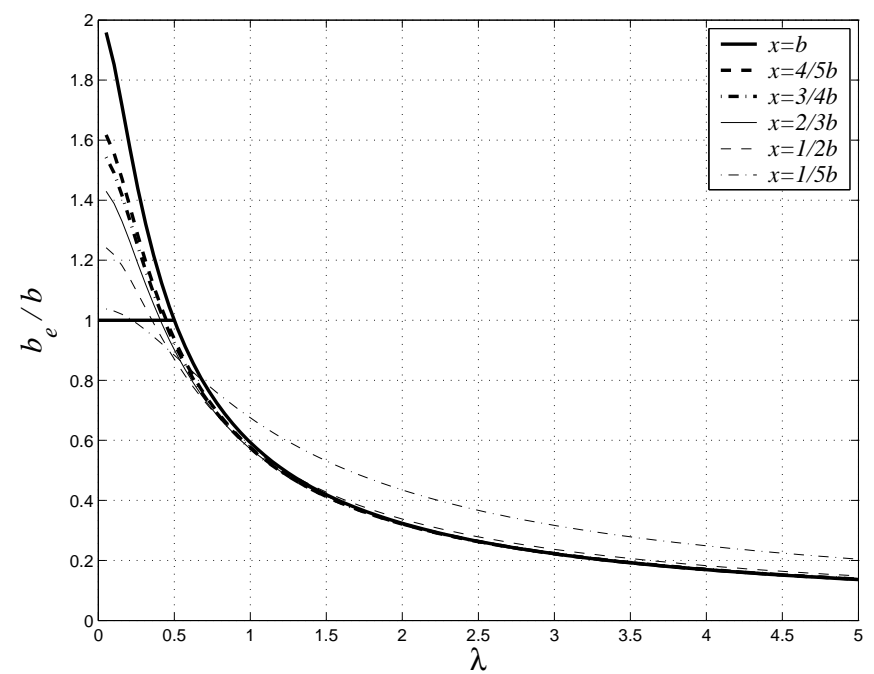

Figure 22. $b_{e} / b$ as a Function of $\lambda$ for Different Values of $X$ 


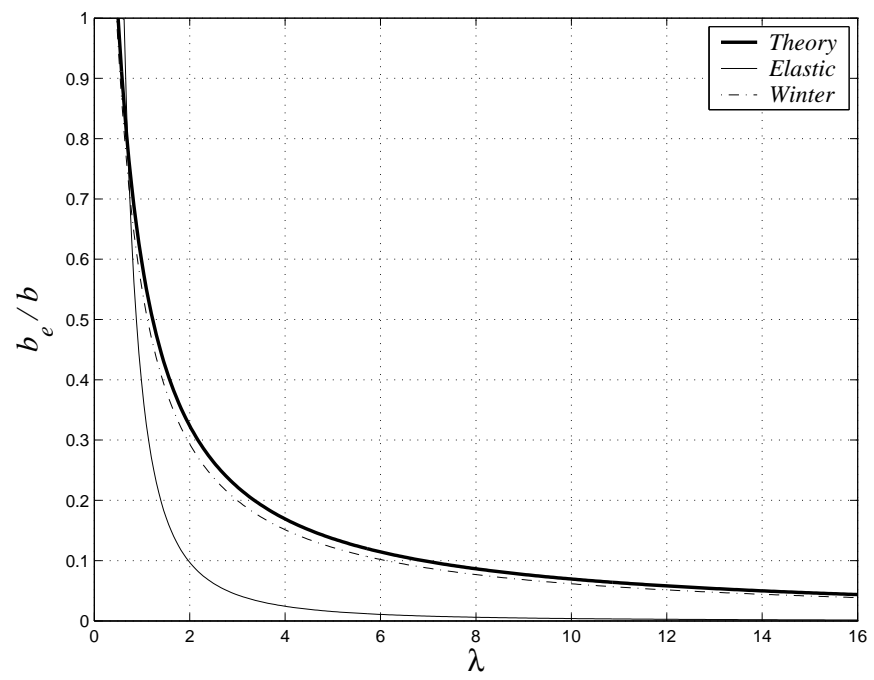

Figure $23 . b_{e} / b$ as a Function of $\lambda$

\subsection{Comparison with Experimental Results}

Figure 24 shows four test series compared with Eq. 70. The figure also shows the elastic solution cf. Eq. 36 and Winter's solution cf. Eq. 71.

In the test series Plates (Bambach and Rasmussen [1]), the specimens were single plates, unconnected to any adjacent elements. These authors applied the test rig suggested by Moxham [9] which enabled them to establish simple support conditions in a reliable way. The series Stub-column, Beams (Kalyanaraman et al. [7]) and I-beams (Winter [15]) all consisted of specimens made by bolting or welding U-sections together.

In Figure 25, the correlation between theory and test is shown. For all tests, a mean value of 0.877 and a standard deviation of $15.7 \%$ are obtained. For the separate test series, the following results are obtained:

- Bambach \& Rasmussen, Plates: Mean 0.981, standard deviation $8.5 \%$.

- Kalyanaraman et al., Stub-column: Mean 0.717, standard deviation 6.2 \%.

- Kalyanaraman et al., Beams: Mean 0.790, standard deviation $12.1 \%$.

- Winter, I-beams: Mean 0.925, standard deviation $16.7 \%$.

The theory seems to underestimate the post-buckling strength for the tests Stub-column and Beams. Kalyanaraman et al. [7]) stated that the mean value of the elastic buckling coefficient was measured to be around $k=0.85$. This number shows that the longitudinal edges cannot have been simply supported, since for a plate with a simply supported longitudinal edge $k=0.43$. For a fixed edge $k=1.28$ according to the elastic theory, cf. (Timoshenko \& Gere [14]). The test series I-beams is defected by a large scatter. Winter [15] gives several explanations for the large scatter. On the other hand, the test series Plates, with the most reliably established boundary conditions, shows a very good agreement with the theory. 


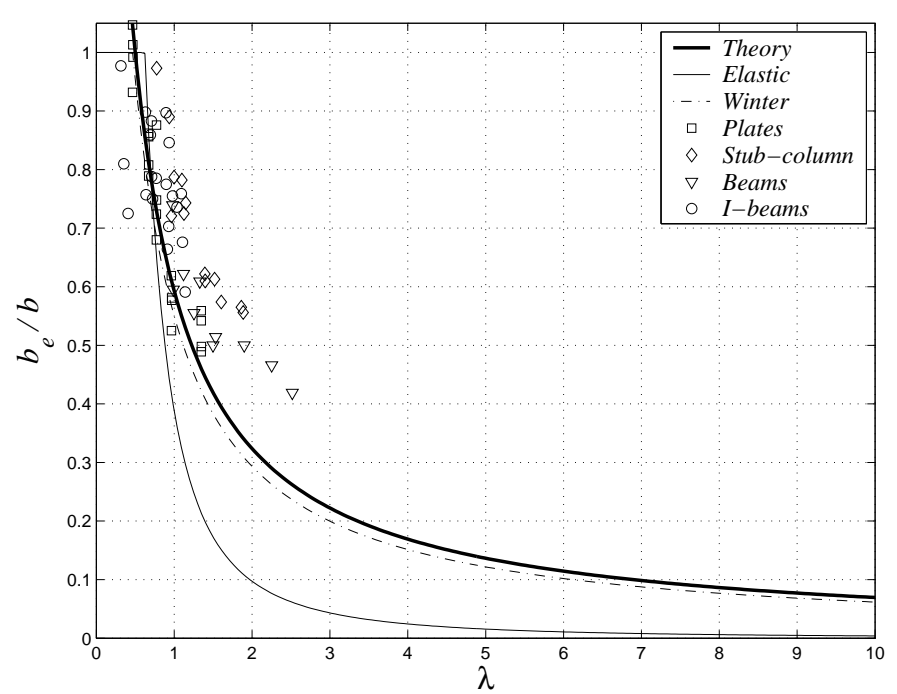

Figure 24. $b_{e} / b$ as a Function of $\lambda$, Theories and Tests

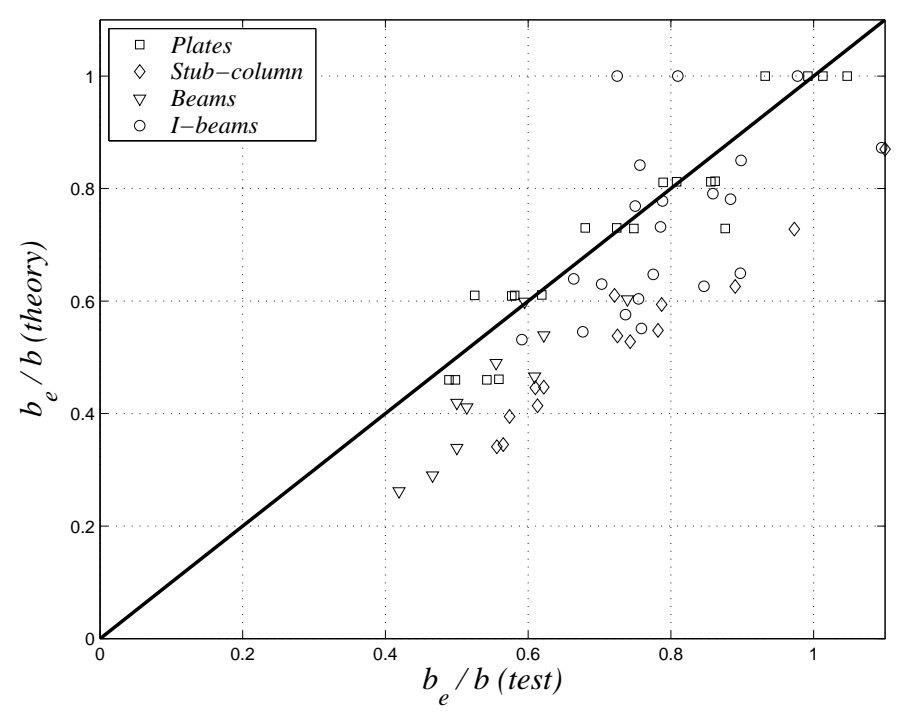

Figure 25. Theory Versus Tests

\subsection{Square Plates}

Square plates in uniaxial compression with one free edge require another failure mechanism than the one shown in Figure 21 valid for rectangular plates.

The optimal mechanism for a square plate is drawn in Figure 26. It has the free parameter, $X$, which is set at $X=1 / 2 b$. The plate is again subjected to uniaxial compression, $n_{p}=t f_{y}$, along an effective width, $b_{e}$, close to the support.

In this case, two work equations must be derived; one corresponding to $b_{e}<X$ and one corresponding to $b_{e}>X$, respectively.

The deflection at maximum load, $u_{m}$, is taken as the deflection of a beam in the direction of the uniaxial compression with the curvature given by Eq. 30. Thus

$u_{m}=\frac{1}{8} \frac{2 \varepsilon_{y}}{t} b^{2}=\frac{1}{4} \frac{f_{y}}{E} \frac{b^{2}}{t}$ 


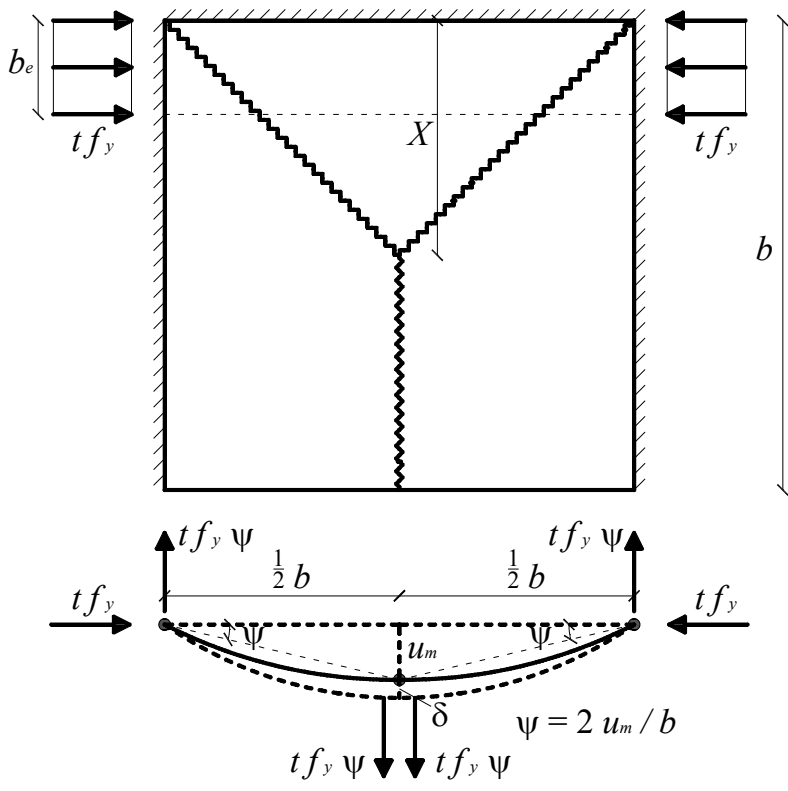

Figure 26. Failure Mechanism for a Square Plate with One Free Edge

The work equations show that the effective width is determined by:

For $b_{e} / b \leq X / b=1 / 2$ :

$\frac{f_{y}}{E} b_{e}^{2}+\frac{t^{2}}{b} b_{e}-\frac{3}{2} t^{2}=0$

$\Rightarrow$

$\frac{b_{e}}{b}=-\frac{1}{2} \lambda^{-2}+\sqrt{\frac{1}{4} \lambda^{-4}+\frac{3}{2} \lambda^{-2}}$

For $b_{e} / b \geq X / b=1 / 2$ :

$\left(\frac{f_{y}}{E} b+\frac{t^{2}}{b}\right) b_{e}=\frac{3}{2} t^{2}+\frac{1}{4} \frac{f_{y}}{E} b^{2}$

$\Rightarrow$

$\frac{b_{e}}{b}=\frac{\frac{1}{4} \lambda^{2}+\frac{3}{2}}{\lambda^{2}+1}$

In the above equations, $\lambda$ is given by Eq. $35, b$ is the total width, $t$ the thickness, $E$ Young's modulus and $f_{y}$ is the yield stress.

Eq. 74 and Eq. 76 are shown in Figure 27 together with the elastic buckling solution, Eq. 36, and Winter's solution, Eq. 71. 


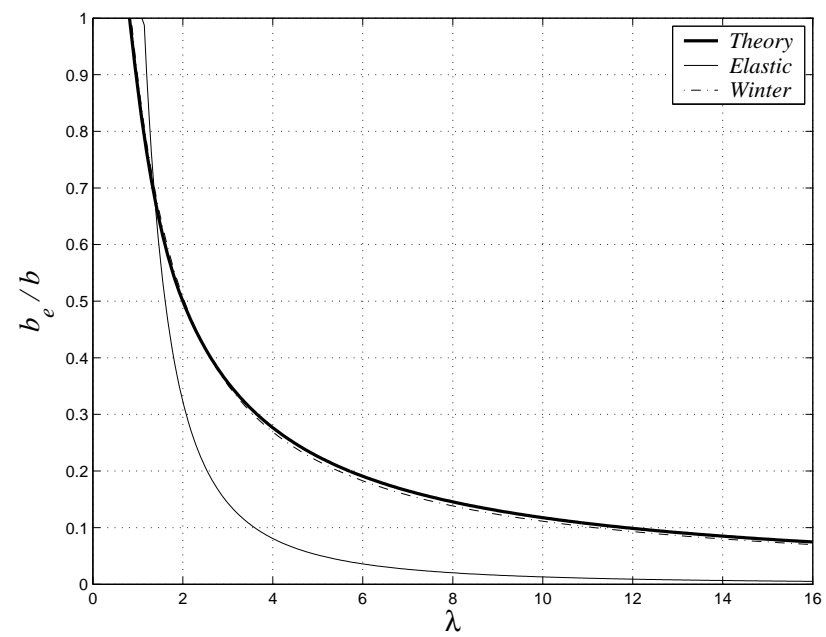

Figure 27. $b_{e} / b$ as a Function of $\lambda$

Here, the buckling coefficient $k=1.43$ is applied, cf. (Timoshenko and Gere [14]), and Poisson's ratio is taken to be $v=0.30$. Again, the theoretical solution gives almost the same result as Winter's semi-empirical solution. Unfortunately, no tests have been found in the literature to verify the theory. This however is not particularly important, since square plates with one free edge are seldom used in practice.

\section{IMPERFECTIONS}

As previously mentioned residual stresses will have no influence on the calculations according to the theory of plasticity.

Approximately imperfections may be taken into account by adding an initial deflection to the deflection used in the above calculations. Thus it is tacitly assumed that the imperfection has the same form as the deflected shape used before.

When considering rectangular plates supported along all edges, the deflection at maximum load is determined by, cf. Eq. 31,

$u_{m}=\frac{1}{4} \frac{f_{y}}{E} \frac{b^{2}}{t}+u_{i}$

where $u_{i}$ is the initial deflection, $f_{y}$ the yield stress, $E$ Young's modulus, $b$ the total width and $t$ is the thickness. Solving the work equation as shown in Section 3.1, leaves the result

$$
\frac{b_{e}}{b}=\frac{-\lambda^{-2}+\sqrt{\lambda^{-4}+4 \lambda^{-2}\left(4 \lambda^{-2} \frac{u_{i}}{t}+1\right)}}{4 \lambda^{-2} \frac{u_{i}}{t}+1}
$$

For an imperfection proportional to the thickness, the term, $u_{i} / t$, will be a constant.

Another way of taking imperfections and other unknown parameters into account is simply to introduce an empirical coefficient, $\mu$, in the deflection formula. The deflection at maximum load 
may then be written

$u_{m}=\mu \frac{1}{4} \frac{f_{y}}{E} \frac{b^{2}}{t}$

The effective width is found to be

$\frac{b_{e}}{b}=-\frac{1}{\mu} \lambda^{-2}+\sqrt{\frac{1}{\mu^{2}} \lambda^{-4}+4 \frac{1}{\mu} \lambda^{-2}}$

Imperfections may explain the large deviations in the tests by Sechler (Winter [15]), both from Winter's formula and the present theoretical estimate.

In Figure 25, it was shown that the tests by Sechler coincided closely with the theoretical estimate for small values of $b_{e} / b$, i.e. large values of $\lambda$, while for low values of $\lambda$, the deviation was large. For the tests by Sechler shown in Figure 25, the mean value is 1.202. By requiring the mean value 1.0 and by applying Eq. 79 to find the imperfection coefficient, $\mu=1.52$ is found. The correlation between the tests and the theory, cf. Eq. 80, with $\mu=1.52$ is shown in Figure 28. In this way, a smaller deviation is achieved for low values of $\lambda$, but the scatter is still large.

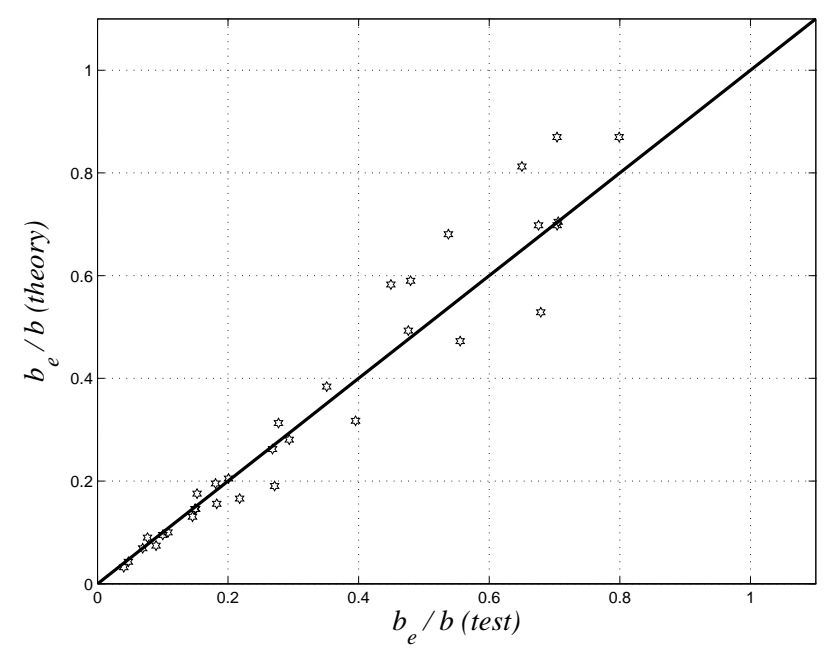

Figure 28. Theory Versus Tests for $\mu=1.52$

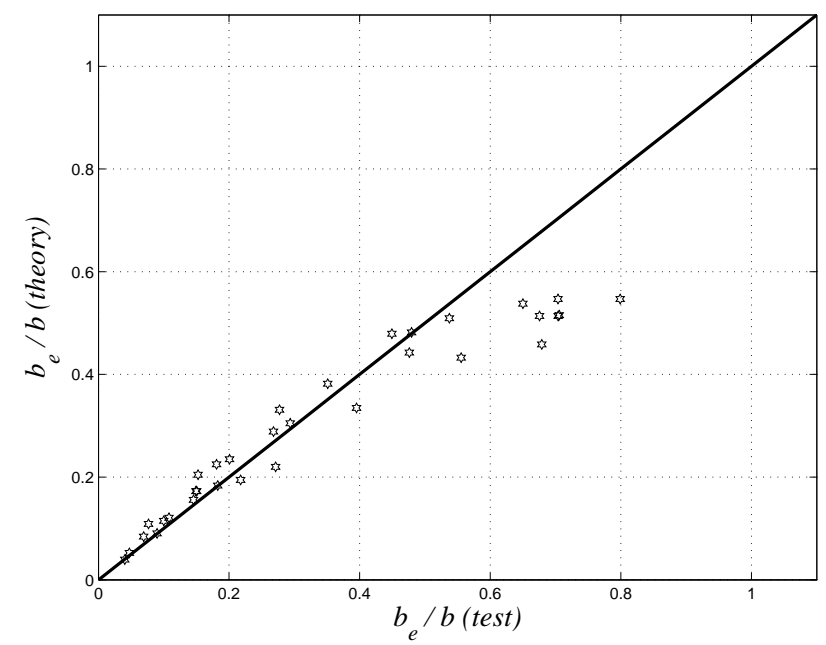

Figure 29. Theory Versus Tests for $u_{i} / t=1.94$ 
Unfortunately, the report by Sechler [13] referred to by Winter has not yet been available to the authors. From Winter's reference it is only possible to deduce the value of $\lambda$, while the thickness of the specimens remains unknown. By applying Eq. 78 and require the mean value $1.0, u_{i} / t=1.94$ is found. The correlation between the tests and the theory with $u_{i} / t=1.94$, cf. Eq. 78 , is shown in Figure 29.

It appears that the effect is small for large values of $\lambda$, and large for low values of $\lambda$, which is precisely the requirement for removing the discrepancy. However, the effect on the result for low values of $\lambda$ seems to be too great. Thus by applying Eq. 40, the theory overestimates the load-carrying capacity for low values of $\lambda$, while the opposite is achieved by applying Eq. 78 . Hence it is not possible to verify Eq. 78 by these tests.

Apart from initial imperfections, there may of course be other explanations for the large deviations found in the Sechler tests. In tests, it is very difficult to correctly establish the ideal support conditions. The supports require the four edges of the initial mid-plane of the plate to remain in the same plane at all times. A solution was suggested by Schuman and Back [12] for the case of single plate specimens. In their tests, the specimens were, as mentioned above, supported by V-grooves, which cause any initial curvature of the edges to increase, which may again cause failure at a lower load than otherwise expected. The effect will be relatively larger for the thicker specimens. Therefore problems related to V-grooves might also explain the deviations in the Sechler tests. However, this is quite hypothetical, since at the moment it is not known if this kind of support was even used in the tests.

\section{OTHER APPLICATIONS OF THE THEORY}

The simple theory developed above may be extended to apply to a large number of practically important cases.

Firstly, an external lateral load may be taken into account by simply adding the work done by the lateral load to the external work, calculated as above. Further, biaxial compressive loads may be treated in the same manner without difficulties. Stiffeners and the compression flange in plate girders may be calculated by formulae given above.

Fixed supports may be treated by adding the contribution from the negative yield line at the fixed supports to the dissipation. However, the deflection at maximum load must also be changed, so that it corresponds to the fixed boundary conditions.

Considering two cases of long rectangular plates with fixed unloaded edges (one fixed unloaded edge and both unloaded edges fixed), the failure mechanisms may be as shown in Figure 30 and 31 , respectively. In both cases, the plate may be subdivided into square plates as shown in the figures. Here the deflection at maximum load is given by Eq. 31 . The load-carrying capacity for a plate with one fixed unloaded edge is then

$$
\frac{b_{e}}{b}=-\lambda^{-2}+\sqrt{\lambda^{-4}+5 \lambda^{-2}}
$$

The load-carrying capacity for a plate with both unloaded edges being fixed equals 


$$
\frac{b_{e}}{b}=-\lambda^{-2}+\sqrt{\lambda^{-4}+6 \lambda^{-2}}
$$

Eq. 81 and 82 are shown in Figure 32 and 33, respectively. The figures also show the results determined be Winter's formulae. Again, the results presented coincide closely with Winter's formulae.

Finally, it is probably also possible to calculate in-plane bending loads. However, other mechanisms have to be used in this case.

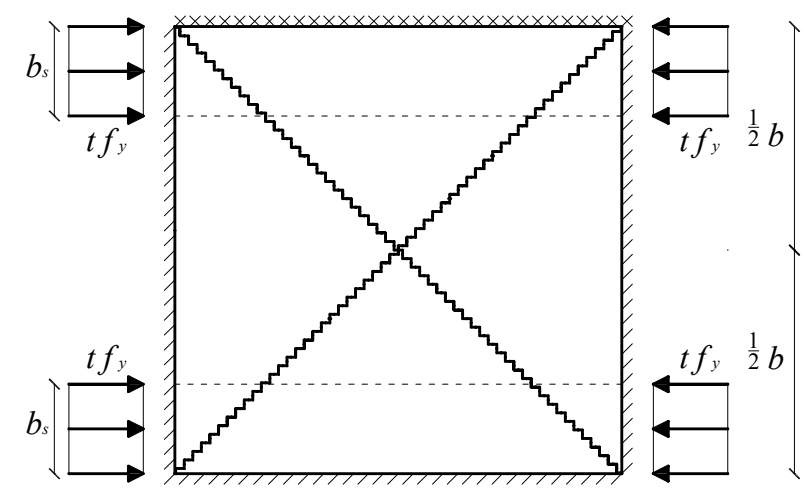

Figure 30. Failure Mechanism for a Plate with One Fixed Unloaded Edge

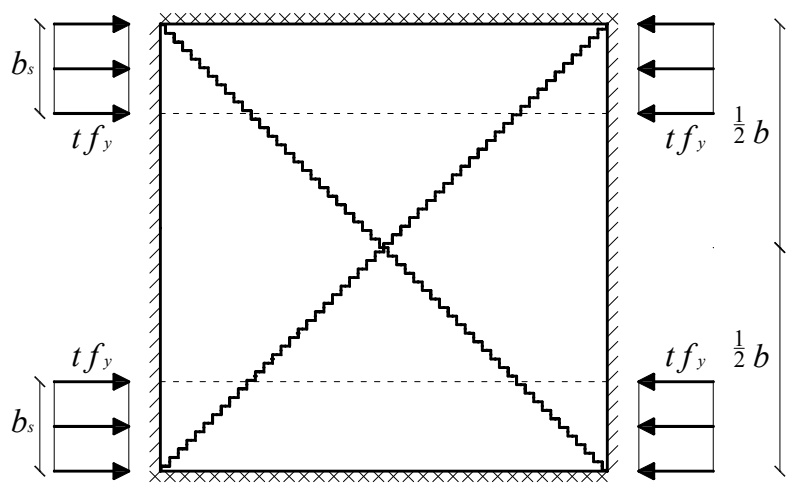

Figure 31. Failure Mechanism for a Plate with Both Unloaded Edges Fixed

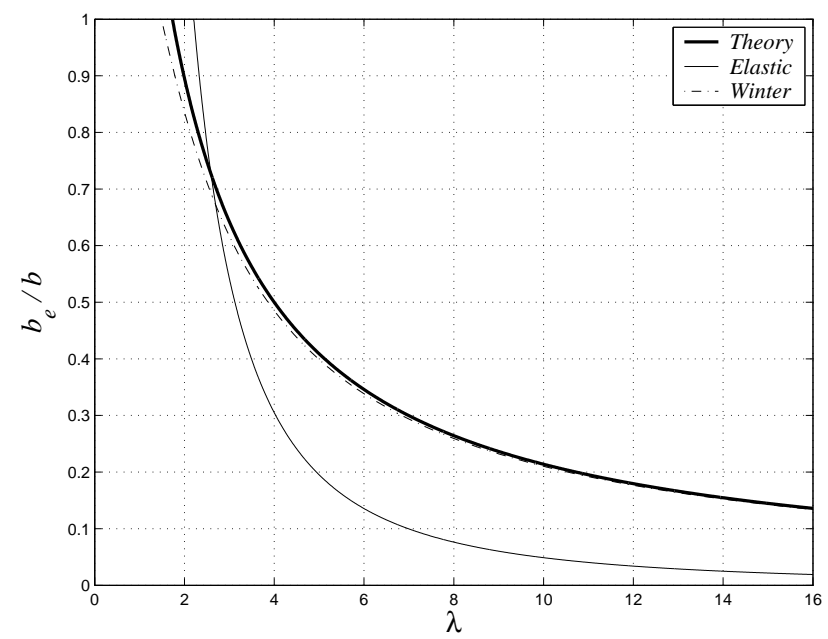

Figure 32. $b_{e} / b$ as a Function of $\lambda$, Fixed Supports Along One Unloaded Edge 


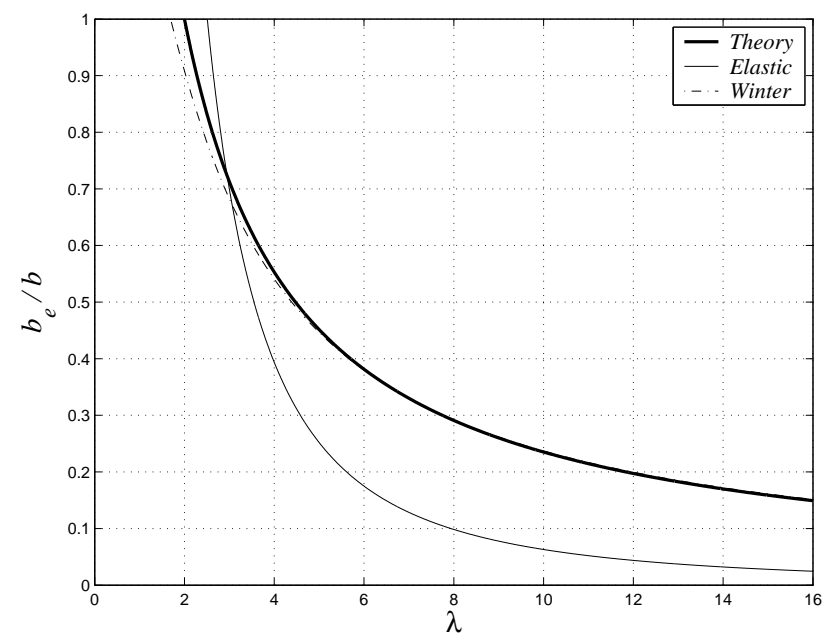

Figure 33. $b_{e} / b$ as a Function of $\lambda$, Fixed Supports Along Both Unloaded Edges

\section{CONCLUSION}

It is shown that extremely simple estimates of the post-buckling strength of plates with in-plane loading may be obtained by using plastic solutions for the deflected shape. This shape must be known or estimated before the calculation can be carried out. It seems that useful estimates of the deflected shape may be found using simple formulae from beam theory and plate theory.

Initially, the calculation procedure is illustrated by simple solutions for columns. Solutions for plates are derived for the two practically important cases: Plates supported along all edges and plates with one free edge. In both cases, the theory is applied on both square and rectangular plates. Furthermore, it is shown how imperfections may be taken into account. Finally, it is shortly explained how the theory may be extended to a large number of other practically important cases. The results have been compared with the well-known formulae of Winter and with tests. The agreement in both cases is very good.

\section{NOTATION}

$\begin{array}{ll}a & \text { plate length } \\ b & \text { plate width } \\ b_{e} & \text { total effective width } \\ b_{s} & \text { width of effective strip } \\ f_{y} & \text { yield stress } \\ k & \text { elastic buckling coefficient } \\ m & \text { bending capacity of a yield line per unit length; fictitious moment } \\ m_{b} & \text { bending moment per unit length in a yield line } \\ m_{p} & \text { plastic yield moments per unit length } \\ n & \text { normal force per unit length } \\ n_{p} & \text { load-carrying capacity per unit length in pure compression or tension } \\ m_{p x}, m_{p y} & \text { plastic yield moment in the } x \text {-direction and the } y \text {-direction, respectively } \\ p & \text { lateral load per unit area } \\ t & \text { thickness } \\ u, u_{A}, u_{B} & \text { deflection; deflection at point } A \text { and } B, \text { respectively } \\ u_{i} & \text { deflection from imperfections }\end{array}$




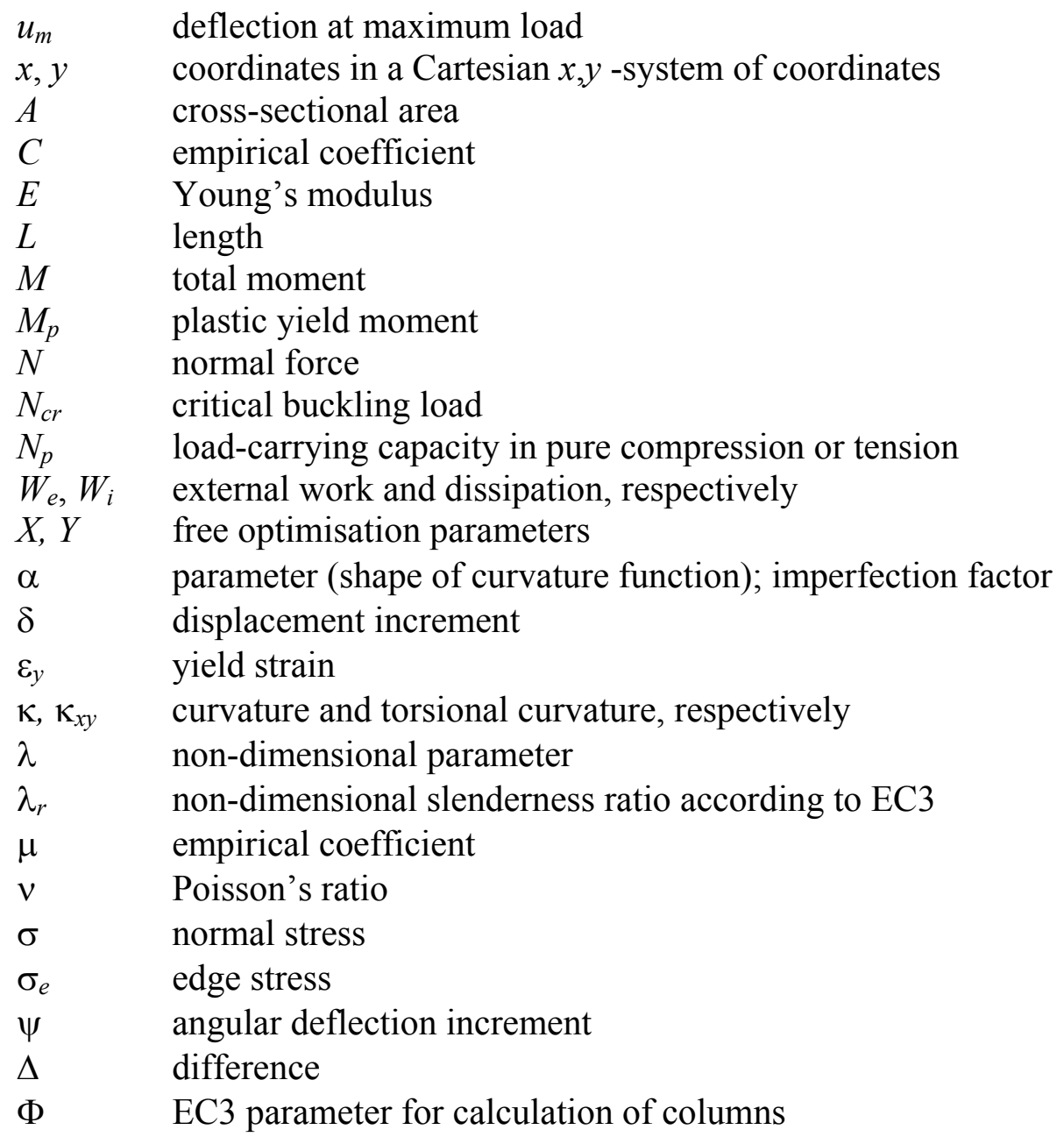

\section{REFERENCES}

[1] Bambach, M.R. and Rasmussen, K.J.R., "Tests on Unstiffened Plate Elements under Combined Compression and Bending”, J. Struct. Eng. ASCE, 2004, Vol. 130, No. 10, pp. 1602-1610.

[2] Bryan, G.H., "On the Stability of a Plane Plate under Thrusts in Its Own Plane, with Applications to the Buckling of the Sides of a Ship", Proc. Lond. Math. Soc. 1891, Vol. 22.

[3] European Committee for Standardisation (EC3 1993), "Eurocode 3: Design of Steel Structures - Part 1-1: General Rules and Rules for Buildings", EN 1993-1-1, Brussels: CEN.

[4] European Committee for Standardisation (EC3 2005), "Eurocode 3: Design of Steel Structures - Part 1-5: Plated Structural Elements", ENV 1993-1-5, Brussels: CEN.

[5] Hiriyur, B.K.J. and Schafer, B.W., Yield-Line Analysis of Cold-Formed Steel Members", Submitted to International Journal of Steel Structures, August 2004.

[6] Johansen, K.W., "Brudlinieteorier", Copenhagen: Gjellerup, 1943. English translation: Johansen, K.W., "Yield Line Theories", London: Cement and Concrete Association, 1962.

[7] Kalyanaraman, V., Winter, G. and Pekoz, T., "Unstiffened Compressed Elements", J. Struct. Div. ASCE, 1977, Vol. 103, No. 9, pp. 1833-1848.

[8] Kármán, T. von, Sechler, E.E. and Donnell, L.H., "The Strength of Thin Plates in Compression", Trans. ASME, 1932, Vol. 54, pp. 53-57.

[9] Moxham, K.E., "Buckling Tests on Individual Welded Steel Plates in Compression", Report CUED/C-Struct/TR.3, Cambridge: University of Cambridge, 1971. 
[10] Murray, N.W., "Introduction to the Theory of Thin-Walled Structures," Oxford: Clarendon Press, Oxford Science Publications, 1984.

[11] Nielsen, M.P., "Limit Analysis and Concrete Plasticity", 2nd ed., Boca Raton, Florida: CRC Press, 1998.

[12] Schuman, L. and Back, G., "Strength of Rectangular Flat Plates under Edge Compression", Technical Report No. 356: National Advisory Committee for Aeronautics, 1930.

[13] Sechler, E.E., "The Ultimate Strength of Thin Flat Sheet in Compression", Publication No. 27, Pasadena: Guggenheim Aeronautics Laboratory, California Inst. of Technology, 1933.

[14] Timoshenko, S.P. and Gere, J.M., "Theory of Elastic Stability", 2nd ed., New York: McGraw-Hill Book Co., Inc., 1961.

[15] Winter, G., "Strength of Thin Steel Compression Flanges", Trans. ASCE, 1947, Paper No. 2305, Vol. 112, pp. 527-554.

[16] Zhao, X.L., "Yield Line Mechanism Analysis of Steel Members and Connections", Structural Analysis and CAD, Prog. Struct. Engng. Mater., 2003, Vol. 5, pp. 252-262. 


\title{
AN ASSESSMENT OF BEAM-TO-COLUMN ENDPLATE AND BASEPLATE JOINTS INCLUDING THE AXIAL-MOMENT INTERACTION
}

\author{
A.A. Del Savio ${ }^{1, *}$, D.A. Nethercot ${ }^{2}$, P.C.G.S. Vellasco ${ }^{3}$, L.R.O. de Lima ${ }^{3}$, \\ S.A.L. Andrade ${ }^{4}$ and L.F. Martha ${ }^{4}$ \\ ${ }^{I}$ Civil Engineering Department, PUC-Rio - Pontifical Catholic University of Rio de Janeiro \& \\ Department of Civil and Environmental Engineering, Imperial College London \\ Rua Marquês de São Vicente, 225, Gávea, Rio de Janeiro, RJ - Brazil, CEP: 22453-900 \\ ${ }^{2}$ Department of Civil and Environmental Engineering, Imperial College London \\ South Kensington Campus, Skempton Building, London SW7 2BU, United Kingdom \\ ${ }^{3}$ Structural Engineering Department, UERJ - State University of Rio de Janeiro \\ Rua São Francisco Xavier, 524, Sala 5018A, Maracanã, Rio, RJ, Brazil, CEP: 20550-900 \\ ${ }^{4}$ Civil Engineering Department, PUC-Rio - Pontifical Catholic University of Rio de Janeiro \\ Rua Marquês de São Vicente, 225, Gávea, Rio de Janeiro, RJ-Brazil, CEP: 22453-900 \\ *(Corresponding author: E-mail: a.delsavio@imperial.ac.uk/delsavio@gmail.com)
}

Received: 26 June 2007; Revised: 30 November 2008; Accepted: 3 December 2008

\begin{abstract}
Knowledge of the moment-rotation characteristic of connections is an essential prerequisite for use of the so called semi-continuous approach to steel and composite frame design. Although the axial force transferred from the beam is frequently low, so that its effect on the moment-rotation characteristic may often be neglect, certain circumstances do exist in which axial compression or tension forces will be sufficiently large that it is no longer reasonable to ignore their influence. Relatively few experimental results have been reported to investigate this effect. A method is presented herein which extends the range of application of available data so as to produce moment-rotation characteristics that implicitly make proper allowance for the presence of significant levels of either tension of compression in the beam. The method depends directly on the availability of a limited amount of data but then uses a corrective process to permit the full range to be covered. The applicably and validity of the proposed methodology is demonstrated through comparisons against several tests on both flush end-plate joints and base plate arrangements.
\end{abstract}

Keywords: Axial versus bending moment interaction; joint behaviour; moment versus rotation curves; rotational stiffness, semi-rigid joints; steel structures

\section{INTRODUCTION}

Under certain circumstances, beam-to-column joints can be subjected to the simultaneous action of bending moments and axial forces. Although, the axial force transferred from the beam is usually low, it may, in some situations attain values that significantly reduce the joint flexural capacity. These conditions may be found in: Vierendeel girder systems (widely used in building construction because they take advantage of the member flexural and compression resistances eliminating the need for extra diagonal members); regular sway frames under significant horizontal loading (seismic or extreme wind); irregular frames (especially with incomplete storeys) under gravity/horizontal loading; and pitched-roof frames.

Moreover, with the recent escalation of terrorist attacks on buildings, the study of progressive collapse of steel framed building has been highlighted, as can be seen in Vlassis et al. [16]. Examples of these exceptional conditions are the cases where structural elements, such as central and/or peripheral columns and/or main beams, are suddenly removed, sharply increasing the joint axial forces. In these situations the structural system, mainly the joints, should be sufficiently robust to prevent the premature failure modes that may lead to progressive structural collapse.

Unfortunately, few experiments considering the interaction bending moment and axial force have been reported in the literature. Additionally, the available experiments are associated with a small 
number of axial force levels and associated bending moment versus rotation curves, $M-\phi$. Thus the, a question still remains for how to incorporate these effects into a structural analysis. There is, therefore, the need of $M-\phi$ curves associated with varying axial force levels, which accurately represent the joint resistance rotational stiffness.

This has led to the development of a relatively simple yet accurate approach to predict any moment versus rotation curve from tests that include the axial versus bending moment interaction. The evaluation and validation of this methodology is executed against a range of available experimental tests for flush endplate joints (Simões da Silva et al., [13]) and base plate joints (Guisse et al. [4]). It is worth highlighting that this methodology is not only restricted to the use of experiments, but can also be applied to results obtained analytically, empirically, mechanically and numerically.

Since this methodology is exclusively based on the use of $M$ - $\phi$ curves, the bending moment versus axial force interactions are intrinsically incorporated, it can be easily implemented into a nonlinear semi-rigid joint finite element formulation because it does not change the element formulation, only requiring a rotational stiffness update procedure.

\subsection{Component Method}

The component method entails the use of relatively simple joint mechanical models, based on a set of rigid links and spring components. The component method - introduced in Eurocode 3 [3] - can be used to determine the joint's resistance and initial stiffness. Its application requires the identification of active components, the evaluation of the force-deformation response of each component (which depends on mechanical and geometrical properties of the joint) and the subsequent assembly of the active components for the evaluation of the joint moment versus rotation response.

Nowadays, using the Eurocode 3 [3] component method, it is possible to evaluate the rotational stiffness and moment capacity of semi-rigid joints when subject to pure bending. However, this component method is not yet able to calculate these properties when, in addition to the applied moment, an axial force is also present. Eurocode 3 [3] suggests that the axial load may be disregarded in the analysis when its value is less than 5\% of the beam's axial plastic resistance, but provides no information for cases involving larger axial forces. Although, the component method does not consider the presence of an axial force, its general principles could be used in these situations. The main reason for this is related to the fact that the Eurocode 3 [3] is based on the use of a series of force versus displacement relationships - which only depend on the component's axial force level - to characterize any individual component behaviour.

\subsection{Background: Experimental and Theoretical Models}

The study of the semi-rigid characteristics of beam-to-column joints and their effects on frame behaviour can be traced back to the 1930s, Li et al. [6]. Since then, a large amount of experimental and theoretical work has been conducted both on the behaviour of the joints and on their effects on complete frame performance. 
Despite the large number of available experiments, they do not cover the full range of interest. As an alternative to tests, other methods have been proposed to predict bending moment versus rotation curves. These procedures range from a purely empirical curve fitting of test data, passing through ingenious behavioural, analogy and semi-empirical techniques, to comprehensive finite element analysis, Nethercot \& Zandonini [10].

Recently, several researchers have paid special attention to joint behaviour under combined bending moment and axial force. Guisse et al. [4] carried out experiments on twelve column bases, six with extended and six with flush endplates. Wald and Svarc [17] tested three flush endplate beam-to-beam joints and two extended endplate beam-to-column joints; however there is no reference to tests made without axial forces, which is necessary as a basis to study the influence of axial force on the joint structural response. Lima et al. [8] and Simões da Silva et al. [13] performed tests on eight flush and seven extended endplate joints. The investigators concluded that the presence of the axial force modifies their structural response and, therefore, should be considered in the joint structural design.

Regarding the theoretical models, Table 1 summarises the mechanical models that have been developed since 1993 to predict the behaviour of beam-to-column joints under bending moment and axial force.

Table 1. Summary of Mechanical Models Used to Predict the Joint Behaviour Subjected to Bending Moments and Axial Forces

\begin{tabular}{ll}
\hline Authors (date) & Type of Joint \\
\hline Wales \& Rossow [18] & Double web cleat connections \\
Madas [9] & Flexible endplate, double web angle and \\
top and seat angle connections
\end{tabular}

Despite the continuous development and improvement of these analytical models there are still limitations in the prediction of the moment-rotation curves, mainly related to the joint initial stiffness for different axial force levels. Usually, the severity of this problem increases when joints are subjected to tensile axial forces and it is commonly related to the ability of these models to deal with moment-axial interaction, consequently changing the compressive centre, before the first component yields.

With the aim of providing an alternative method to the mechanical models for prediction of moment-rotation curves for any axial force level from a limited set of experiments, the next section presents a detailed methodology based on the use of Correction Factors initially proposed by Del Savio et al. [2]. 


\section{PREDICTION OF MOMENT-ROTATION CURVES FOR ANY AXIAL FORCE LEVEL FROM A LIMITED SET OF EXPERIMENTS}

\subsection{General Concepts of the Correction Factor}

The Correction Factor was initially proposed by Del Savio et al. [2] to allow for the bending moment versus axial force interaction, by scaling original moment values present in the moment versus rotation curves (disregarding the axial force effect). This strategy shifts this curve up or down depending on the axial force level. However, as it only modifies the bending moment axis, it is not able to fully describe the bending moment versus rotation associated with different axial force levels. This fact is highlighted when the joint is subject to a tensile axial force, where there is a significant difference, mainly, in terms of initial stiffness.

With the aim of improving this basic idea, the Correction Factor has been divided into two parts: one for the moment axis and another for the rotation axis. Both corrections are in principle independent, and do not depend on the moment versus axial force interaction diagram, as was the case for the initial idea presented by Del Savio et al. [2]. It is now only a function of the moment versus rotation curves for different axial force levels.

\subsection{Extension of the Correction Factors for Both Bending Moment and Rotation Axes}

As previously noted, there are two corrections, one to the moment axis and another to the rotation axis. As a general approach, the Correction Factor for the moment axis is evaluated in terms of the bending moment versus rotation curves considering the axial force effect. Using the design bending moment ratio and considering the axial force effect, the Correction Factor for the moment axis, $C F_{M}$, can be evaluated by:

$$
C F_{M}=\frac{M_{\text {int }}}{M_{\text {max }}} \quad \begin{aligned}
& M_{\text {int }}=f\left(M x \phi\left(N_{i}\right)\right) \\
& M_{\text {max }}=f(M x \phi(0.0))
\end{aligned}
$$

where $N_{i}$ is the axial force present in the $i$ interaction; $M x \phi$ or $M-\phi$ is the bending moment versus rotation curve; $M_{\text {int }}$ is the design bending moment for the $M-\phi\left(N_{i}\right)$ curve considering the axial force $N_{i}$; and $M_{\max }$ is the design bending moment for the $M-\phi(0.0)$ curve without axial forces. $M_{\text {int }}$ and $M_{\max }$ can be determined according to Eurocode 3 [3], through the intersection between two straight lines, one parallel to the initial stiffness and another parallel to the $M-\phi$ curve post-limit stiffness, Figure 1.

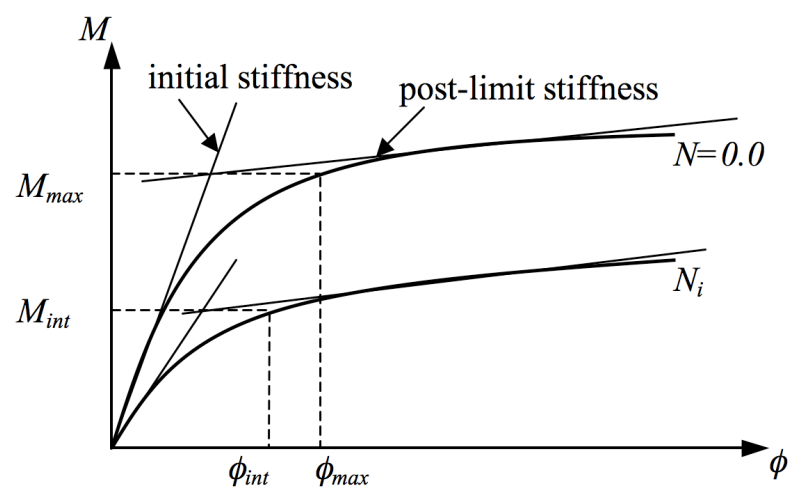

Figure 1. Evaluation of the Design Bending Moments $\left(M_{\text {int }} \& M_{\max }\right)$ and Rotations $\left(\phi_{i n t} \& \phi_{\max }\right)$ 
Similarly, the rotation axis Correction Factor, $C F_{\phi}$ is evaluated using the design rotation ratio, i.e.:

$$
C F_{\phi}=\frac{\phi_{\text {int }}}{\phi_{\max }} \quad \begin{aligned}
& \phi_{\text {int }}=f\left(M x \phi\left(N_{i}\right)\right) \\
& \phi_{\text {max }}=f(M x \phi(0.0))
\end{aligned}
$$

where $\phi_{i n t}$ and $\phi_{\max }$ are the design rotations related respectively to $M_{\text {int }}$ and $M_{\max }$. Both design rotations are found by tracing a horizontal straight line at the design moment level until it reaches the $M-\phi$ curve. At this point a vertical straight line is drawn until it intersects the rotation axis, Figure 1. With the Correction Factors evaluated for both the moment and rotation axes, Eqs. 1 and 2 respectively, they are then incorporated into the joint structural response considering the moment versus axial force interaction by modifying the $M-\phi$ curve for the zero axial force case, i.e.:

$$
\begin{aligned}
& \operatorname{Mx} \phi(0.0) \rightarrow \operatorname{Mx} \phi\left(N_{i}\right) \\
& \operatorname{Mx} \phi\left(N_{i}\right)=\operatorname{Mx} \phi\left(M_{N=0} \times C F_{M}, \phi_{N=0} \times C F_{\phi}\right)
\end{aligned}
$$

Basically, the $M-\phi$ curve point coordinates $M_{N=0}$ and $\phi_{N=0}$ referred to the moment and the rotation axis coordinates, respectively, for the case without axial forces, are multiplied by the Correction Factors $C F_{M}$ and $C F_{\phi}$, respectively.

However, only using a pair of Correction Factors throughout the whole $M-\phi$ curve, for the case without axial forces, does not provide a good approximation to the $M-\phi$ curve considering the axial force, because its response is very sensitive to the adopted initial and post-limit stiffnesses.

This prompted the division of the $M-\phi$ curve into three segments with different pairs of Correction Factors. These divisions were made at two-third, one, and 1.1 times the design bending moment $M_{d}$ as shown in Figure 2.

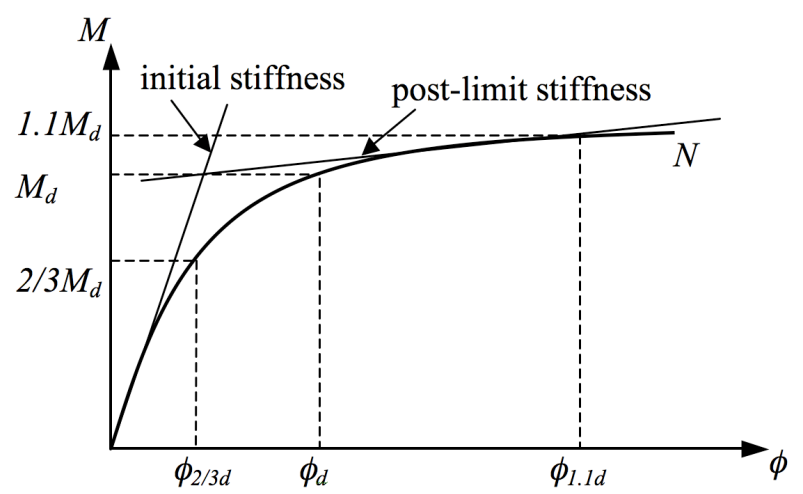

Figure 2. Correction Factor strategy method using a three segment division of the $M-\phi$ curve

With this division, the Correction Factors cannot be applied as presented in Eq. 3. This is justified, in fact, because they would provoke two abrupt variations of stiffness throughout the approximate $M-\phi$ curve at around the point of intersection of the approximate curve with the vertical lines at the points $\phi_{2 / 3 d}$ and $\phi_{d}$, Figure 3. This is due to the use of three different pairs of Correction Factors evaluated according to Eqs. 1 and 2 at two-third, one, and 1.1 times the design bending moment $M_{d}$. 


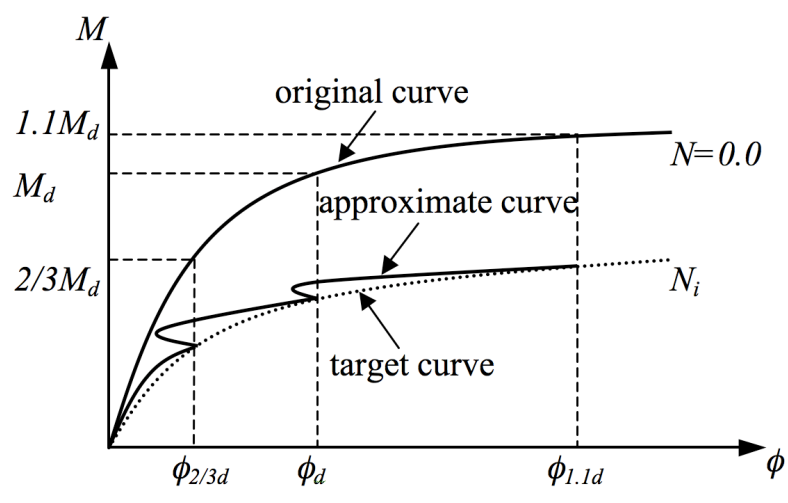

Figure 3. Approximate $M-\phi$ curve using three Correction Factor pairs

\subsection{An Alternative Methodology}

Based on the division of the $M-\phi$ curve into three segments with different pairs of Correction Factors, previously mentioned, in Figure 4, a tri-linear representation for the $M$ - $\phi$ curve was proposed. This method overcomes the problem of abrupt alterations of stiffness presented in Figure 3 as well as guaranteeing an accurate approximation of the $M-\phi$ curve at points: $\left(2 / 3 M_{d}, \phi_{2 / 3 d}\right) ;\left(M_{d}\right.$, $\left.\phi_{d}\right)$ and $\left(1.1 M_{d}, \phi_{1.1 d}\right)$.

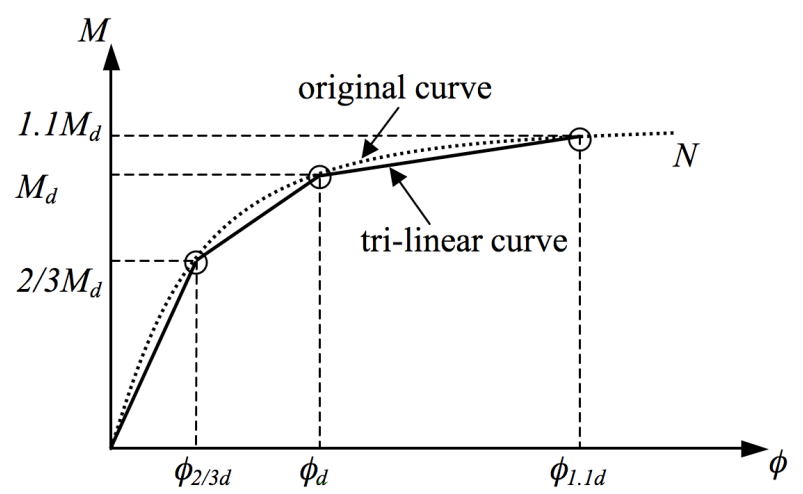

Figure 4. Tri-linear representation of the $M-\phi$ curve methodology

From the tri-linear representation proposed in Figure 4, the bending moments of the target $M-\phi$ curve, associated with a certain axial force level $\left(N_{i}\right)$, can be evaluated by:

$$
\begin{aligned}
& M_{p}=\left(M_{N, p}-M_{0, p}\right) \frac{N_{i}}{N}+M_{0, p} \\
& p=2 / 3 M_{d} ; M_{d} ; 1.1 M_{d} \\
& 0<N_{i} \leq N \rightarrow \text { tensile axial force } \\
& N \leq N_{i}<0 \rightarrow \text { compressive axial force }
\end{aligned}
$$

where the subscript $p$ refers to three analysed points: $2 / 3 M_{d}, M_{d}$, and $1.1 M_{d} ; N$ is the axial force load level associated with the reference $M-\phi$ curve; $N_{i}$ is the axial force load level related to the target $M-\phi$ curve; $M_{p}$ is the bending moment evaluated for the target $M-\phi$ curve at point $p ; M_{N, p}$ is the bending moment on the reference $M-\phi$ curve considering the axial force at point $p$; and $M_{0, p}$ is the bending moment on the reference $M$ - $\phi$ curve without axial forces at point $p$. 
Likewise, the rotations of the evaluated $M-\phi$ curve, for the associated $N_{i}$, can be calculated by:

$$
\begin{aligned}
& \phi_{p}=\left(\phi_{N, p}-\phi_{0, p}\right) \frac{N_{i}}{N}+\phi_{0, p} \\
& p=2 / 3 M_{d} ; M_{d} ; 1.1 M_{d} \\
& 0<N_{i} \leq N \rightarrow \text { tensile axial force } \\
& N \leq N_{i}<0 \rightarrow \text { compressive axial force }
\end{aligned}
$$

where $\phi_{p}$ is the rotation evaluated for the target $M-\phi$ curve at point $p ; \phi_{N, p}$ is the rotation on the reference $M-\phi$ curve considering the axial force at point $p$; and $\phi_{0, p}$ is the rotation on the reference $M-\phi$ curve without axial force effects at point $p$.

The evaluations of the bending moments and rotations proposed in Eqs. 4 and 5, respectively, for prediction of the target $M-\phi$ curve are, in essence, linear interpolations between two reference $M-\phi$ curves - considering and disregarding the axial force - at points: $\left(2 / 3 M_{d}, \phi_{2 / 3 d}\right) ;\left(M_{d}, \phi_{d}\right)$ and $\left(1.1 M_{d}\right.$, $\left.\phi_{1.1 d}\right)$.

\section{APPLICATION OF THE PROPOSED METHODOLOGY}

The main focus of the methodology presented in section 2.3 is to determine $M-\phi$ curves for any axial force level from two reference $M-\phi$ curves. The quality of the obtained approximations depends on the quality of the $M-\phi$ curves used as input to the method. This methodology requires, at least, two $M-\phi$ curves, disregarding and considering either the compressive or tensile axial force effect. However, for a complete behavioural evaluation of the joint three $M-\phi$ curves are necessary: one disregarding the axial force effect; another considering the compressive force effect and finally a third alternative considering the tensile force effect. In this way, it is possible to study the entirely joint structural response given that loading applied to the joint may vary from compression to tension.

In order to explain the application of this method to obtain $M-\phi$ curves for any axial force level, as well as to validate its use, experimental tests carried out by Simões da Silva et al. [13] and Guisse et al. [4] on eight flush endplate joints and twelve column bases have been used.

\subsection{Flush Endplate Joints}

This section evaluates experimental tests carried out by Simões da Silva et al. [13] on eight flush endplate joints. The geometric properties of the flush endplate, the $M-\phi$ curves describing the experimental behaviour of each test, and the bending moment versus axial force interaction diagram are shown in Figures 5 to 7. 


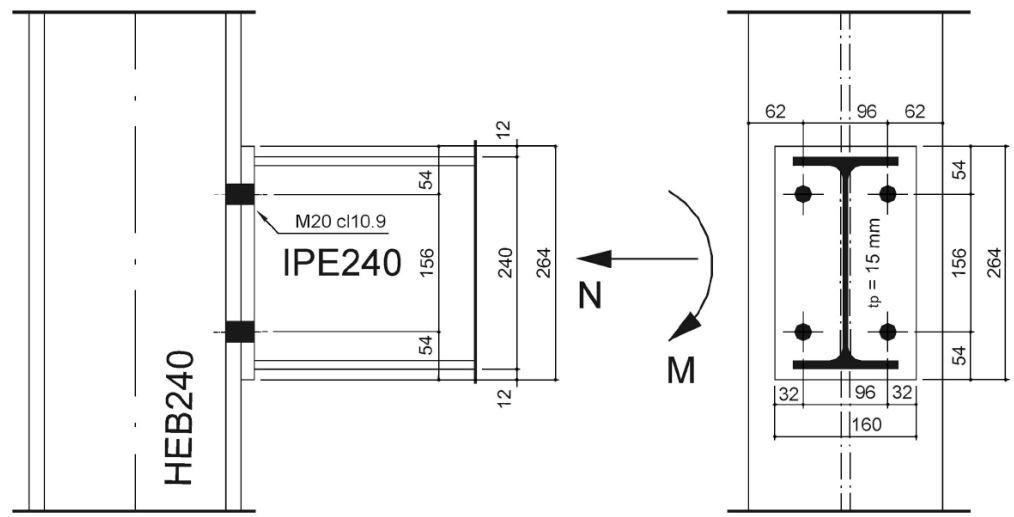

Figure 5. Flush endplate joint layout, Simões da Silva et al. [13]

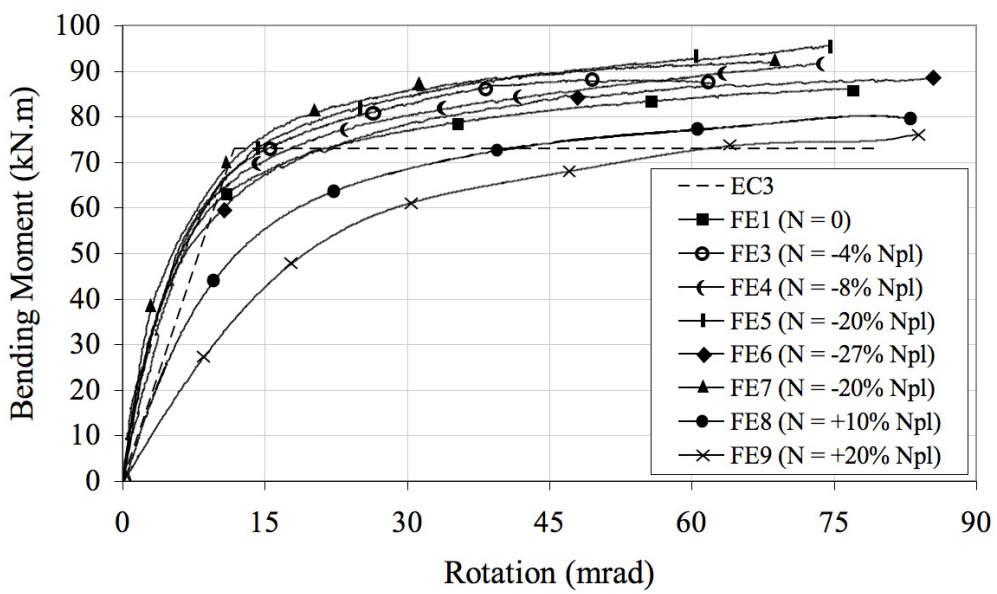

Figure 6. Experimental moment versus rotation curves, Simões da Silva et al. [13]

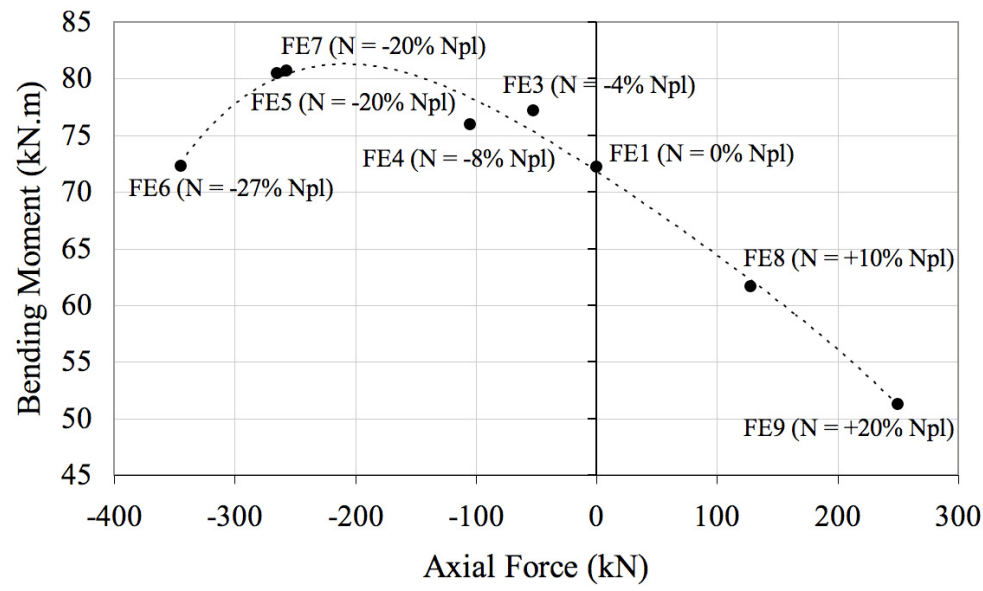

Figure 7. Flush endplate bending moment versus axial force interaction diagram, Simões da Silva et al. [13]

The experimental data, Figure 6, provides the necessary input for the proposed method. The minimum input is composed of two $M-\phi$ curves, disregarding and considering either the tensile or compressive axial force. However, the flush endplate joint, tested by Simões da Silva et al. [2], exhibited a decrease in the moment resistance for the tensile axial forces whilst achieving the highest moment resistance for a compressive axial force equal to $20 \%$ of the beam's axial plastic resistance (see Figure 7, FE7). Three reference $M-\phi$ curves were adopted to demonstrate this joint's 
behaviour relative to the type of axial force: FE1 $(N=0)$; FE7 $(N=-257 \mathrm{kN},-20 \% \mathrm{Npl}$, compressive force), and FE9 $(N=250 \mathrm{kN},+20 \% \mathrm{Npl}$, tensile force), where $\mathrm{Npl}$ is the beam's axial plastic resistance. These three experimental curves and their tri-linear approximations are shown in Figure 8. Additionally, Table 2 presents all the values evaluated for these tri-linear approximations according to Figure 2, where the points for each tri-linear reference $M-\phi$ curve were obtained from the joint design moment, $M_{d}$, which is given by the intersection between two straight lines, one parallel to the initial stiffness and another parallel to the $M-\phi$ curve post-limit stiffness.

Table 2. Values Evaluated for the Reference $M-\phi$ Curves

\begin{tabular}{|c|c|c|c|c|c|c|}
\hline \multirow[b]{2}{*}{ Point } & \multicolumn{2}{|c|}{$\begin{array}{c}F E 1 \\
(N=0.0)\end{array}$} & \multicolumn{2}{|c|}{$\begin{array}{c}F E 7 \\
(N=-257 \mathrm{kN},-20 \% \\
N p l)\end{array}$} & \multicolumn{2}{|c|}{$\begin{array}{c}F E 9 \\
(N=+250 \mathrm{kN},+20 \% \\
N p l)\end{array}$} \\
\hline & $\phi(m r a d)$ & $M(k N m)$ & $\phi(\mathrm{mrad})$ & $M(k N m)$ & $\phi(m r a d)$ & $M(k N m)$ \\
\hline 0 & 0.0 & 0.0 & 0.0 & 0.0 & 0.0 & 0.0 \\
\hline $2 / 3 M_{d}$ & 6.3 & 50.6 & 6.8 & 56.1 & 13.0 & 38.4 \\
\hline$M_{d}$ & 27.6 & 76.0 & 26.8 & 84.1 & 25.8 & 57.7 \\
\hline $1.1 M_{d}$ & 56.1 & 83.5 & 67.3 & 92.2 & 35.0 & 63.5 \\
\hline
\end{tabular}

Tri-linear $M$ - $\phi$ curves, Figure 8 , are used to define paths between each curve at points $2 / 3 M_{d}, M_{d}$ and $1.1 M_{d}$, Figure 9. These paths were used to guide the linear interpolators for bending moments, Eq. 4, and rotations, Eq. 5, throughout the given range of axial force levels to determine the required set of $M-\phi$ curves.

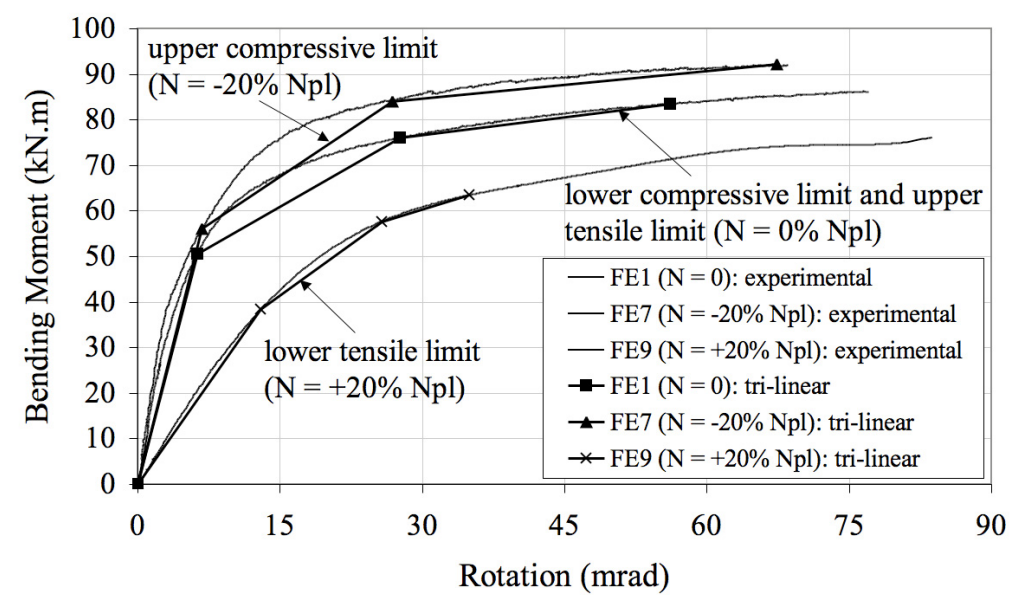

Figure 8. Tri-linear Strategy Used for the Experimental $M-\phi$ Curves

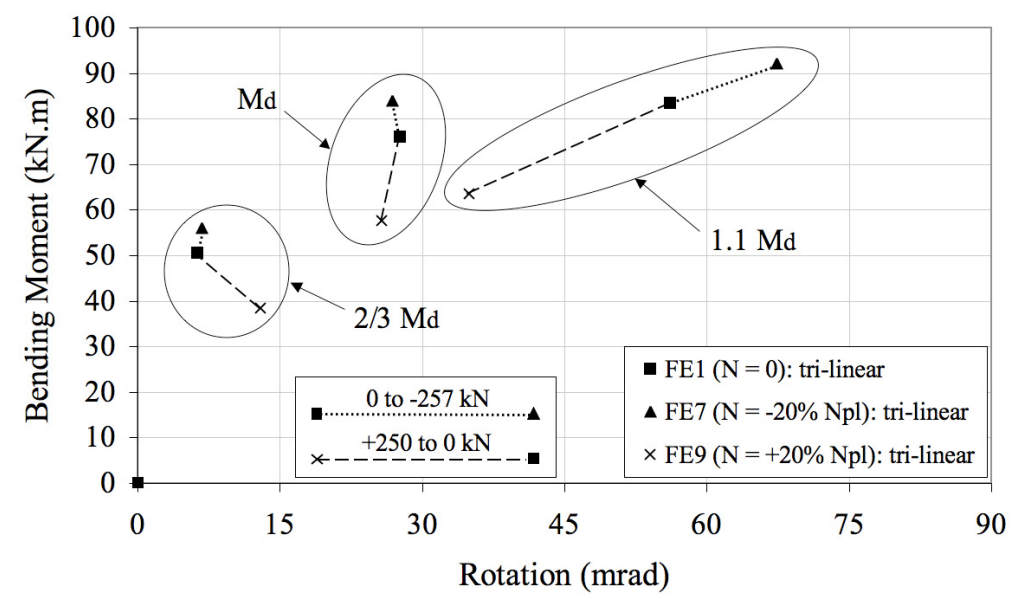

Figure 9. Paths Used to Define the Procedure to

Determine any $M-\phi$ Curve Present within these Limits 
Subsequently, Table 3 depicts the results obtained by using the proposed methodology to predict three experimental $M-\phi$ curves: FE8 for a $10 \%$ tensile force of the beam's axial plastic resistance, FE3 and FE4 for compressive forces of $4 \%$ and $8 \%$, respectively, of the beam's axial plastic resistance. Following this strategy, as an example, Eq. 6 demonstrates how to calculate point $1.1 M_{d}$, Table 3, of the FE8 approximated $M-\phi$ curve. Figures 10 to 12 graphically depict these results. Figure 13 presents the whole set of predicted $M-\phi$ curves utilising this methodology.

Table 3. Values evaluated for three tri-linearly approximated $M-\phi$ curves

\begin{tabular}{|c|c|c|c|c|c|c|}
\hline \multirow[b]{2}{*}{ Point } & \multicolumn{2}{|c|}{$\begin{array}{c}F E 3 \\
\left(N_{i}=-53 k N,-4 \%\right. \\
N p l)\end{array}$} & \multicolumn{2}{|c|}{$\begin{array}{c}F E 4 \\
\left(N_{i}=-105 k N,-8 \% ~ N p l\right)\end{array}$} & \multicolumn{2}{|c|}{$\begin{array}{c}F E 8 \\
\left(N_{i}=+128 k N,+10 \%\right. \\
N p l)\end{array}$} \\
\hline & $\phi(m r a d)$ & $M(k N m)$ & $\phi(m r a d)$ & $M(k N m)$ & $\phi(\mathrm{mrad})$ & $M(k N m)$ \\
\hline 0 & 0.0 & 0.0 & 0.0 & 0.0 & 0.0 & 0.0 \\
\hline $2 / 3 M_{d}$ & 6.4 & 51.8 & 6.5 & 52.9 & 9.7 & 44.4 \\
\hline$M_{d}$ & 27.4 & 77.6 & 27.3 & 79.3 & 26.7 & 66.6 \\
\hline $1.1 M_{d}$ & 58.4 & 85.3 & 60.7 & 87.1 & 45.3 & 73.3 \\
\hline
\end{tabular}

FE8 : $\quad$ Point $=p=1.1 M_{d}$

$M_{p}=\left(M_{N, p}-M_{0, p}\right) \frac{N_{i}}{N}+M_{0, p}=(63.5-83.5) \frac{128.0}{250.0}+83.5=73.3 \mathrm{kNm}$

$\phi_{p}=\left(\phi_{N, p}-\phi_{0, p}\right) \frac{N_{i}}{N}+\phi_{0, p}=(35.0-56.1) \frac{128.0}{250.0}+56.1=45.3 \mathrm{mrad}$

$M_{N, p} ; \phi_{N, p}$ and $N \rightarrow$ Table $2: F E 9$

$M_{0, p}$ and $\phi_{0, p} \quad \rightarrow$ Table $2: F E 1$

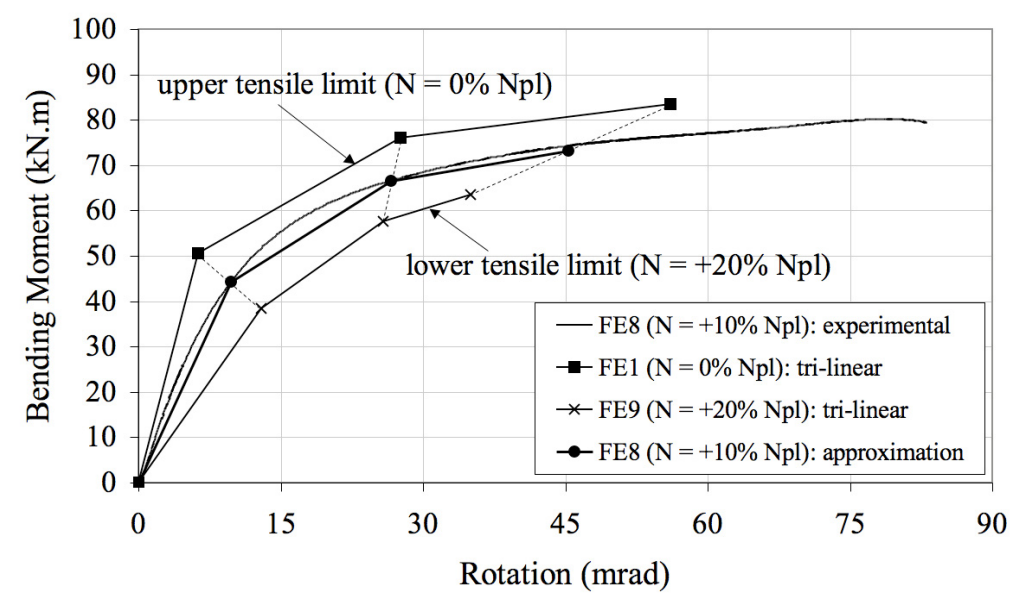

Figure 10. FE8 $M$ - $\phi$ Curve Approximation, Considering a Tensile Force of 10\% of the Beam's Axial Plastic Resistance 


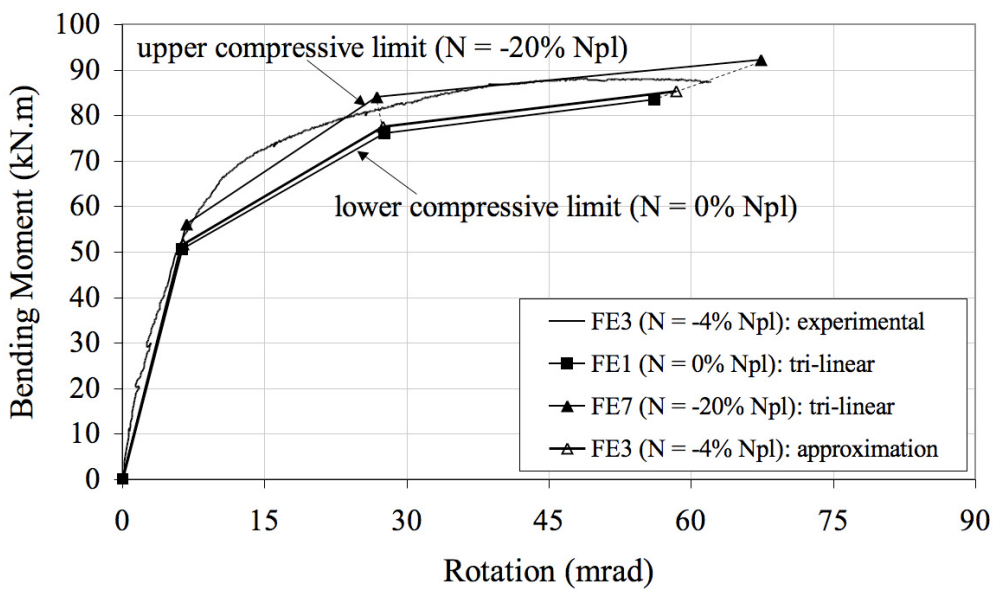

Figure 11. FE3 $M-\phi$ Curve Approximation, Considering a Compressive Force of $4 \%$ of the Beam's Axial Plastic Resistance

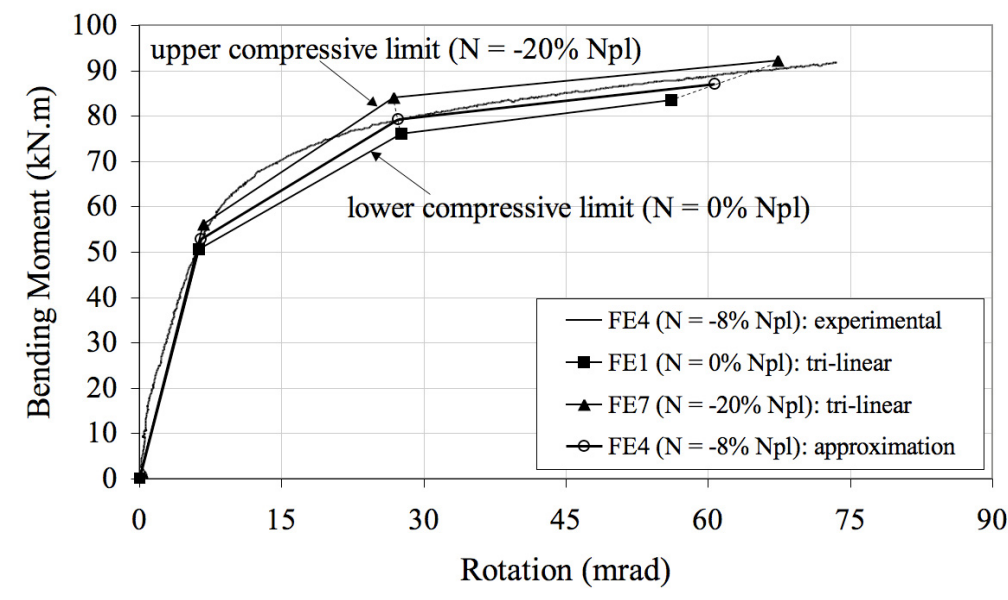

Figure 12. FE4 $M-\phi$ Curve Approximation, Considering a Compressive Force of $8 \%$ of the Beam's Axial Plastic Resistance

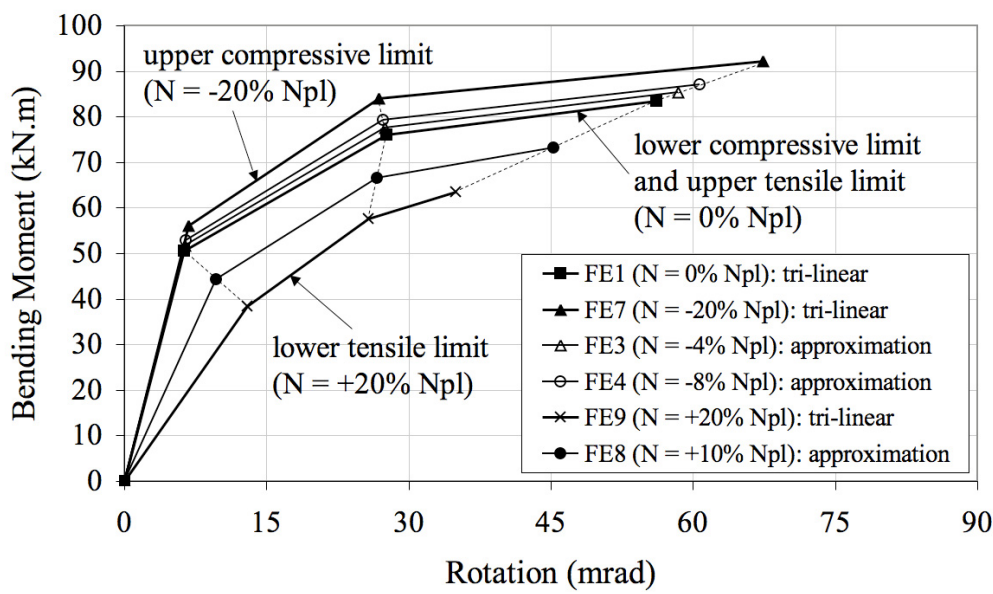

Figure 13. The Whole Set of Predicted $M$ - $\phi$ Curves by Using the Proposed Methodology 


\subsection{Column Bases}

This section presents the evaluation of the experiments performed by Guisse et al. [4] on twelve column base joints. Test configurations with respectively four and two anchor bolts, Figures 14(a) and 14(b), were considered. The steel column profile was a S355 HE160B, whilst the S235 base plates utilised two different thicknesses: $15 \mathrm{~mm}$ and $30 \mathrm{~mm}$. The baseplates are welded to the column with 6 mm fillet welds connected with M20 10-9 anchor bolts.

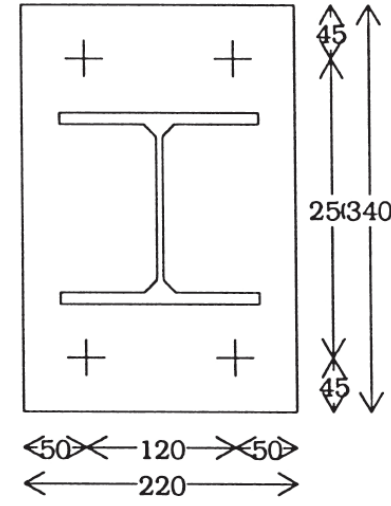

(a) Four anchor bolts

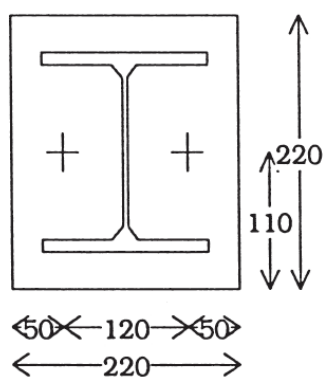

(b) Two anchor bolts

Figure 14. Baseplate Configurations, Guisse et al. [4]

Table 4 presents the set of the tested column bases and Figures 15 to 18 show the experimental $M-\phi$ curves obtained by Guisse et al. [4].

Table 4. Nomenclature of the Tests and their Parameters, Guisse et al. [4]

\begin{tabular}{lccc}
\hline \multicolumn{1}{c}{ Name } & Anchor bolts & Plate thickness $(\mathbf{m m})$ & Normal force $(\boldsymbol{k N})$ \\
\hline$P C 2.15 .100$ & 2 & 15 & 100 \\
$P C 2.15 .600$ & 2 & 15 & 600 \\
$P C 2.15 .1000$ & 2 & 15 & 1000 \\
$P C 2.30 .100$ & 2 & 30 & 100 \\
$P C 2.30 .600$ & 2 & 30 & 600 \\
$P C 2.30 .1000$ & 2 & 30 & 1000 \\
$P C 4.15 .100$ & 4 & 15 & 100 \\
$P C 4.15 .400$ & 4 & 15 & 400 \\
$P C 4.15 .1000$ & 4 & 15 & 1000 \\
$P C 4.30 .100$ & 4 & 30 & 100 \\
$P C 4.30 .400$ & 4 & 30 & 400 \\
$P C 4.30 .1000$ & 4 & 30 & 1000 \\
\hline
\end{tabular}

Since the experiments used only compressive forces, two reference $M-\phi$ curves were adopted for each set of tests related to the axial forces of 100 and $1000 \mathrm{kN}$. The experimental $M-\phi$ curves and their tri-linear approximations are shown in Figures 15 to 18. Additionally, Table 5 presents all the values evaluated for these tri-linear approximations according to Figure 2. 
Table 5. Values Evaluated for the Reference $M-\phi$ Curves

\begin{tabular}{|c|c|c|c|c|c|c|c|c|c|c|c|c|c|c|c|c|}
\hline \multirow{3}{*}{$\frac{5}{2}$} & \multicolumn{8}{|c|}{$P C 2$} & \multicolumn{8}{|c|}{$P C 4$} \\
\hline & \multicolumn{2}{|c|}{15.100} & \multicolumn{2}{|c|}{15.1000} & \multicolumn{2}{|c|}{30.100} & \multicolumn{2}{|c|}{30.1000} & \multicolumn{2}{|c|}{15.100} & \multicolumn{2}{|c|}{15.1000} & \multicolumn{2}{|c|}{30.100} & \multicolumn{2}{|c|}{30.1000} \\
\hline & $\phi$ & $M$ & $\phi$ & $\boldsymbol{M}$ & $\phi$ & $M$ & $\phi$ & $M$ & $\phi$ & $M$ & $\phi$ & $M$ & $\phi$ & $\boldsymbol{M}$ & $\phi$ & $M$ \\
\hline 0 & 0.0 & 0.0 & 0.0 & 0.0 & 0.0 & 0.0 & 0.0 & 0.0 & 0.0 & 0.0 & 0.0 & 0.0 & 0.0 & 0.0 & 0.0 & 0.0 \\
\hline $2 / 3 M_{d}$ & 21.0 & 21.3 & 9.0 & 41.3 & 25.0 & 17.3 & 10.5 & 46.0 & 10.0 & 32.0 & 16.0 & 62.7 & 12.0 & 46.0 & 11.0 & 72.0 \\
\hline$M_{d}$ & 40.0 & 32.0 & 30.0 & 62.0 & 44.0 & 26.0 & 29.0 & 69.0 & 28.0 & 48.0 & 40.0 & 94.0 & 33.0 & 69.0 & 35.0 & $\begin{array}{c}108 . \\
0\end{array}$ \\
\hline $1.1 M$ & 50.0 & 352 & 60.0 & 62.0 & 51.0 & 28.6 & 62.0 & 75.9 & 43.0 & 52.8 & 60.0 & 94.0 & 50.0 & 75.9 & 64.0 & 108. \\
\hline
\end{tabular}

Note: $M$ in $\mathrm{kNm}$ and $\phi$ in $\mathrm{mrad}$.

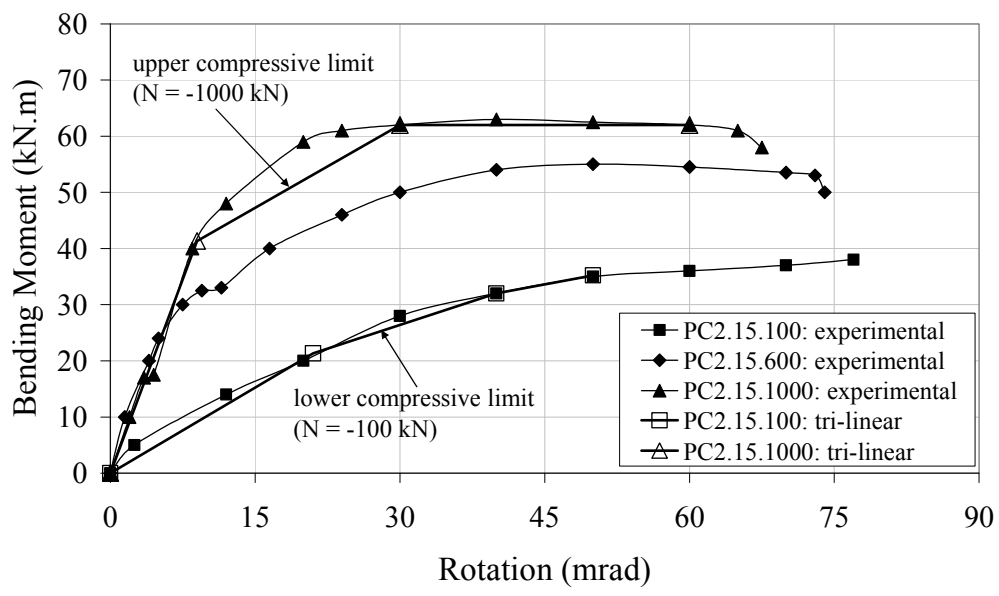

Figure 15. PC2.15 Experimental $M-\phi$ Curves and the Tri-linear Reference $M-\phi$ Curves

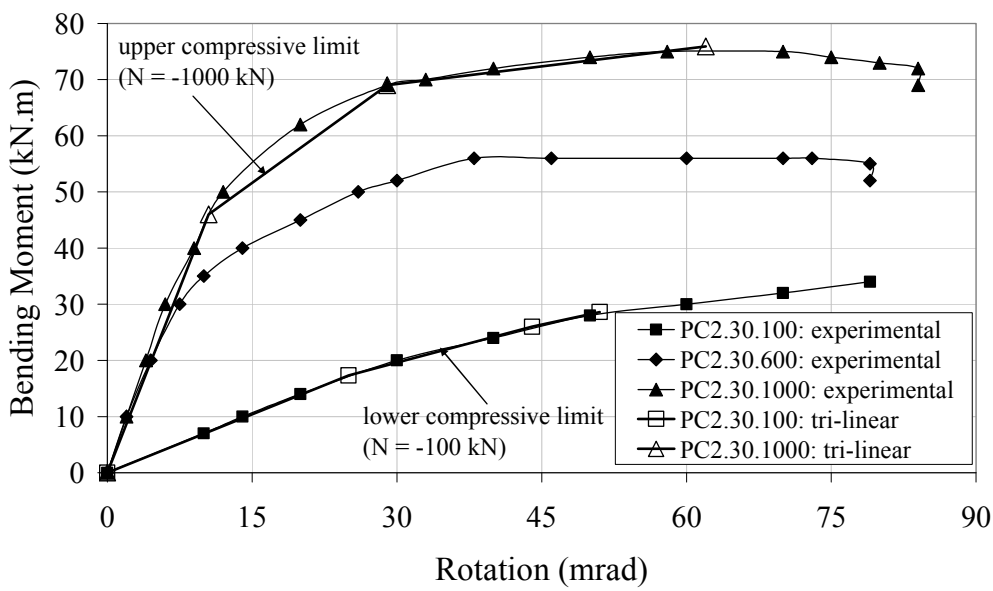

Figure 16. PC2.30 Experimental $M-\phi$ Curves and the Tri-linear Reference $M-\phi$ Curves 


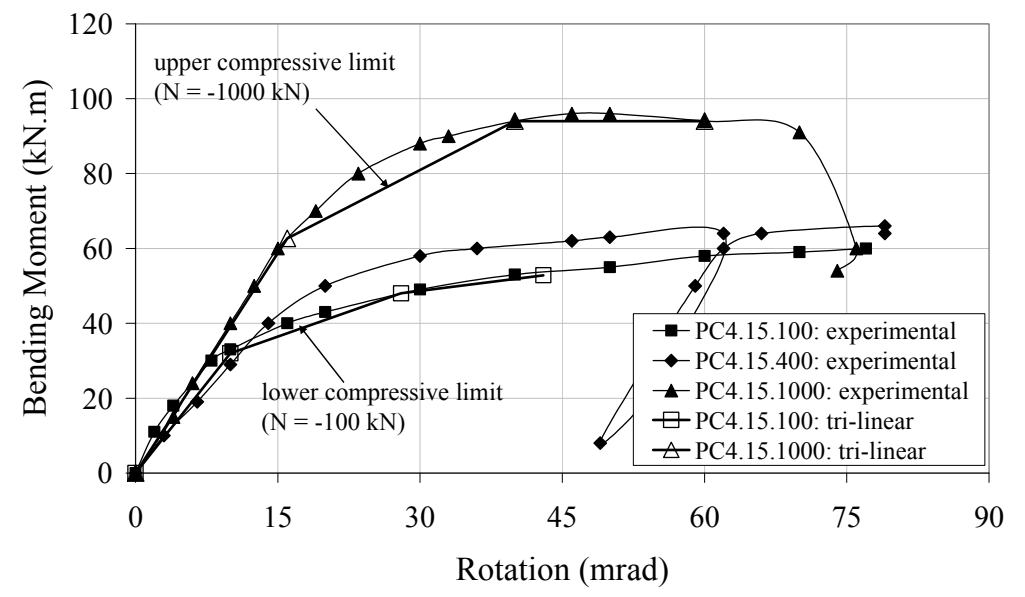

Figure 17. PC4.15 Experimental $M-\phi$ Curves and the Tri-linear Reference $M-\phi$ Curves

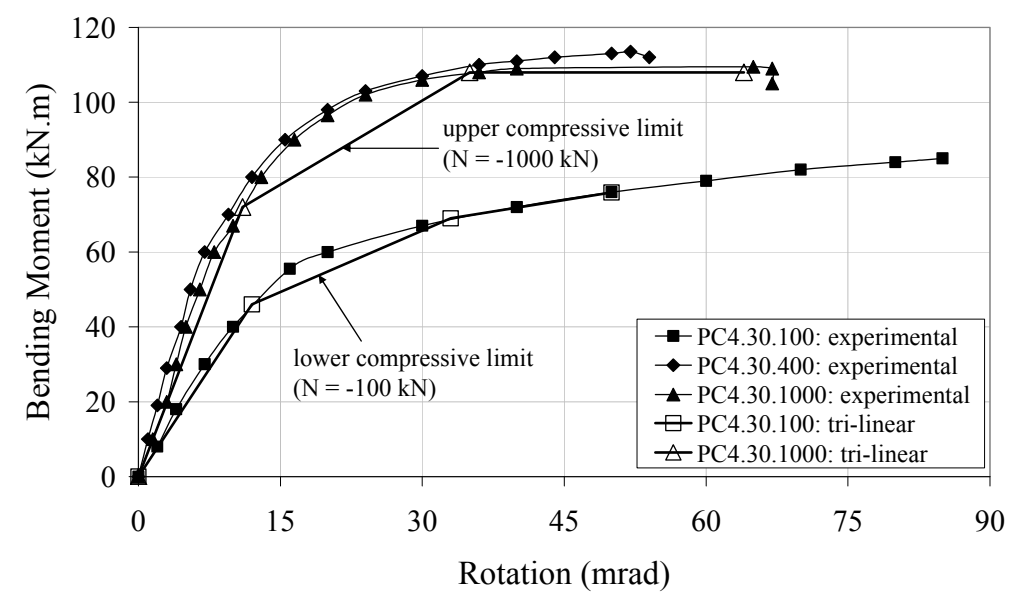

Figure 18. PC4.30 Experimental $M-\phi$ Curves and the Tri-linear Reference $M$ - $\phi$ Curves

Table 6 presents the results obtained by using the proposed method, with the aid of Eqs. 4 and 5, to predict four experimental $M-\phi$ curves: PC2.15.600; PC2.30.600; PC4.15.400 and PC4.30.400. Since there is no reference to experimental $M-\phi$ curve disregarding the axial force effect the experimental $M-\phi$ curves related to axial loads of $100 \mathrm{kN}$ are adopted for the base $M-\phi$ curves. This strategy implies that the axial force load $N$, associated with the reference $M-\phi$ curve, used in Eqs. 4 and 5, was decreased by $100 \mathrm{kN}$. Equation 7 demonstrates how to calculate point $2 / 3 M_{d}$, Table 6 , of the PC2.30.600 approximated $M-\phi$ curve. Finally, Figures 19 to 22 graphically show these results.

Table 6. Values Evaluated for Three Tri-linearly Approximated $M-\phi$ Curves

\begin{tabular}{cccccccccc}
\hline & \multicolumn{4}{c}{$\boldsymbol{P C 2}$} & \multicolumn{4}{c}{$\boldsymbol{P C 4}$} \\
$\Xi$ & $\mathbf{1 5 . 6 0 0}$ & $\mathbf{3 0 . 6 0 0}$ & $\mathbf{1 5 . 4 0 0}$ & \multicolumn{3}{c}{$\mathbf{3 0 . 4 0 0}$} \\
$\mathbf{Q}$ & $\boldsymbol{\phi}$ & $\boldsymbol{M}$ & $\boldsymbol{\phi}$ & $\boldsymbol{M}$ & $\boldsymbol{\phi}$ & $\boldsymbol{M}$ & $\boldsymbol{\phi}$ & $\boldsymbol{M}$ \\
\hline 0 & 0.0 & 0.0 & 0.0 & 0.0 & 0.0 & 0.0 & 0.0 & 0.0 \\
$2 / 3 M$ & 13.0 & 34.7 & 15.3 & 36.4 & 12.7 & 45.6 & 11.6 & 57.6 \\
$d$ & & & & & & & & \\
$M d$ & 33.3 & 52.0 & 34.0 & 54.7 & 33.3 & 68.4 & 33.9 & 86.3 \\
$1.1 M d$ & 56.7 & 53.1 & 58.3 & 60.1 & 50.6 & 71.1 & 56.2 & 90.2 \\
\hline
\end{tabular}

Note: $M$ in $\mathrm{kNm}$ and $\phi$ in mrad. $N_{i}$ is equal to $600 \mathrm{kN}$ for PC2 and $400 \mathrm{kN}$ for PC4. 
PC2.30.600: Point $=p=2 / 3 M_{d}$

$M_{p}=\left(M_{N, p}-M_{0, p}\right) \frac{N_{i}}{N}+M_{0, p}=(46.0-17.3) \frac{600.0}{1000.0-100.0}+17.3=36.4 \mathrm{kNm}$

$\phi_{p}=\left(\phi_{N, p}-\phi_{0, p}\right) \frac{N_{i}}{N}+\phi_{0, p}=(10.5-25.0) \frac{600.0}{1000.0-100.0}+25.0=15.3 \mathrm{mrad}$

$M_{N, p} ; \phi_{N, p}$ and $N \rightarrow$ Table $5: P C 2.30 .1000$

$M_{0, p}$ and $\phi_{0, p} \quad \rightarrow$ Table $5: F C 2.30 .100$

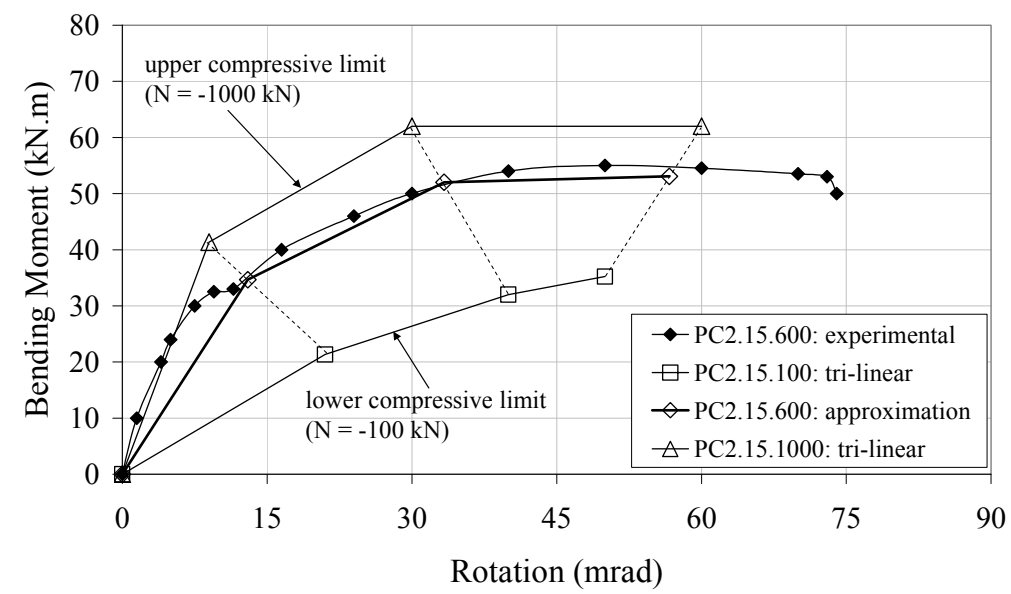

Figure 19. PC2.15.600 $M-\phi$ Curve Approximation

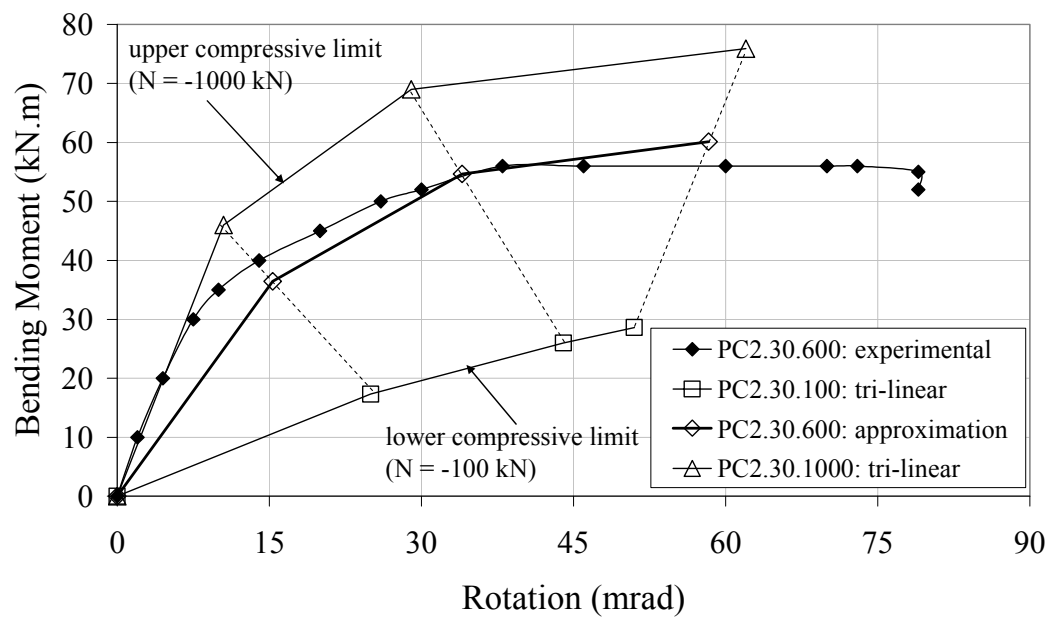

Figure 20. PC2.30.600 $M-\phi$ Curve Approximation 


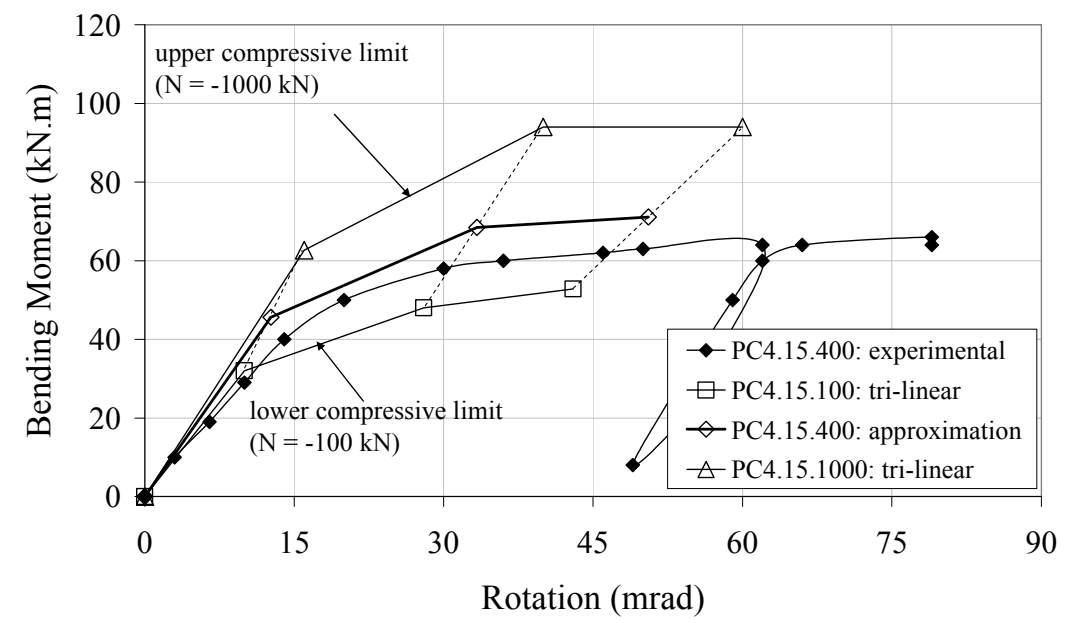

Figure 21. PC4.15.400 $M-\phi$ Curve Approximation

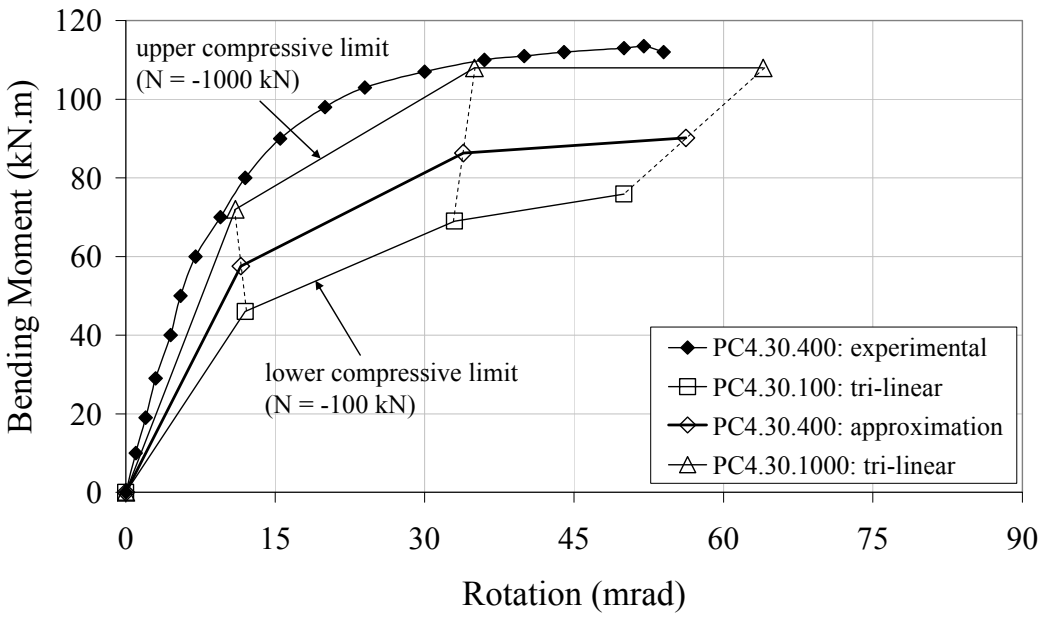

Figure 22. PC4.30.400 $M-\phi$ Curve Approximation

\section{RESULTS AND DISCUSSION}

Three flush endplate joint experimental $M-\phi$ curves, Simões da Silva et al. [13], were evaluated and are depicted in Figures 10-13. They were used to validate the proposed methodology presented in section 2.3 as well as to demonstrate its application.

Figure 10 illustrates an approximation for the FE8 $M-\phi$ curve that considers a tensile force equal to $10 \%$ of the beam's axial plastic resistance. This approximation was obtained from two tri-linear $M-\phi$ curves, disregarding and considering a tensile force of $20 \%$ of the beam's axial plastic resistance. This approximation was very close to the FE8 $M-\phi$ test curve, Table 7.

Figures 11 and 12, present approximations for FE3 and FE4 $M-\phi$ curves that respectively consider compressive forces of $4 \%$ and $8 \%$ of the beam's axial plastic resistance. These approximations were obtained from two tri-linear $M-\phi$ curves, disregarding and considering a compressive force of $20 \%$ of the beam's axial plastic resistance. The approximation for FE4 $M-\phi$ curve, Figure 12, was relatively close to the experimental curve, Table 7 . However, for FE3 $M-\phi$ curve, Figure 11, the obtained response was not as good, underestimating the joint flexural capacity by $11 \%$, Table 7 . This was due to the behaviour of this experimental curve when compared to the others. It is possible to observe in Figure 7 that there is an increase in the flush endplate joint moment capacity from FE1 $M-\phi$ curve $(N=0 \% N p l)$ to FE7 $M-\phi$ curve $(N=-20 \% N p l)$. However, within this range, 
with a $4 \%$ beam's compressive plastic resistance the flexural capacity is larger than the maximum moment obtained with the $8 \%$ test. Following this increasing tendency in the joint flexural capacity registered from FE1 $(N=0 \% \mathrm{Npl})$ to FE7 $(N=-20 \% \mathrm{Npl})$, the maximum moment obtained with FE4 $(N=-8 \% N p l)$ should be larger than FE3 $(N=-4 \% N p l)$. A possible reason for this perturbation in the experimental results might be related to problems with the FE3 experimental test such as measuring errors or assembly eccentricities.

In general, the predictions of the $M-\phi$ curves using the methodology proposed in section 2.3 provided accurate correlations with the test curves from Simões da Silva et al. [13] as can be seen in Table 7.

Table 7. Comparisons between the experimental and the proposed methodology in terms of initial stiffness and design moment capacity for flush endplate joints

\begin{tabular}{ccccccccc}
\hline \multirow{2}{*}{ Tests } & \multicolumn{3}{c}{ Initial Stiffness $(\boldsymbol{k N m} / \mathbf{r a d})$} & \multicolumn{4}{c}{ Design Moment $(\boldsymbol{k N m})$} \\
& Appr & Exp & Appr/Exp & \% & Appr & Exp & Appr/Exp & \% \\
\hline FE3 $(N=-4 \%$ Npl) & 8097 & 10132 & 0.80 & 20 & 74 & 83 & 0.89 & 11 \\
FE4 $(N=-8 \% ~ N p l)$ & 8147 & 10903 & 0.75 & 25 & 75 & 75 & 1.00 & 0 \\
FE8 $(N=+10 \%$ & 4568 & 5403 & 0.85 & 15 & 64 & 68 & 0.94 & 6 \\
\hline
\end{tabular}

Note: Negative percentage means overestimated value in \% whilst positive percentage indicates underestimated value in \%. Joint design moment was determined according to Eurocode 3 [3], through the intersection between two straight lines, one parallel to the initial stiffness and another parallel to the moment-rotation curve post-limit stiffness.

Regarding the tests performed by Guisse et al. [4], four baseplate experimental $M-\phi$ curves were evaluated and are presented in Figures 19 to 22. Figure 19 draws the prediction of PC2.15.600 M- $\phi$ curve for a compressive force of $600 \mathrm{kN}$, by using two reference $M-\phi$ curves: PC2.15.100 and PC2.15.1000. It is possible to note the very close approximation reached at the evaluated points: $2 / 3 M_{d}, M_{d}$ and $1.1 M_{d}$. On the other hand, the initial stiffness was rather erratic being estimated to be $44 \%$ (Table 8) smaller than the experimental one. This fact occurred because the point $2 / 3 M_{d}$, i.e. the first point of the approximated $M-\phi$ curves, is located above the onset point of physical separation of the plate and the concrete in the tensile zone. Therefore, the point $2 / 3 M_{d}$ was just able to capture the initial stiffness final change not considering the initial stiffness before the separation of the steel plate and the concrete base.

Figure 20 presents the PC2.30.600 $M$ - $\phi$ curve approximation for a compressive force of $600 \mathrm{kN}$, by utilising the reference $M-\phi$ curves: PC2.30.100 and PC2.30.1000. A reasonable approximation was obtained for this $M-\phi$ curve, however the initial stiffness was underestimated by $32 \%$ and the flexural capacity was slightly under predicted by $5 \%$, Table 8 .

Figure 21 demonstrates the PC4.15.400 $M-\phi$ curve prediction for a compressive force of $400 \mathrm{kN}$, by employing the base $M-\phi$ curves: PC4.15.100 and PC4.15.1000. A good correlation between the experimental tests and numerical results was obtained. Unlike the others results, the initial stiffness and the design bending moment were over predicted by $26 \%$ and $3 \%$, respectively.

Finally, Figure 22 presents the estimation of the PC4.30.400 $M-\phi$ curve for a compressive force of $400 \mathrm{kN}$, by having as basis PC4.30.100 and PC4.30.1000 M- $\phi$ curves. This case did not produce an accurate prediction of the $M-\phi$ curve, Table 8 . However, this fact may be justified due to the occurrence of the column end section yielding as well as column flange local plate buckling. In others words, the column capacity was reached before achieving the base plate joint flexural capacity. 
Table 8. Comparisons between the experimental and the proposed methodology in terms of initial stiffness and design moment capacity for baseplate joints

\begin{tabular}{ccccccccc}
\hline \multirow{2}{*}{ Tests } & \multicolumn{3}{c}{ Initial Stiffness $(\boldsymbol{k N m} / \mathbf{r a d})$} & \multicolumn{3}{c}{ Design Moment $(\boldsymbol{k N m})$} \\
& Appr & Exp & Appr/Ex & \% & Appr & Exp & Appr/Exp & \% \\
\hline$P C 2.15 .600$ & 2667 & 4800 & 0.56 & 44 & 52 & 54 & 0.96 & 4 \\
$P C 2.30 .600$ & 2377 & 3500 & 0.68 & 32 & 53 & 56 & 0.95 & 5 \\
$P C 4.15 .400$ & 3602 & 2857 & 1.26 & -26 & 65 & 63 & 1.03 & -3 \\
$P C 4.30 .400$ & 4981 & 9091 & 0.55 & 45 & 85 & 111 & 0.77 & 23 \\
\hline
\end{tabular}

Note: Negative percentage means overestimated value in \% whilst positive percentage indicates underestimated value in \%. Joint design moment was determined according to Eurocode 3 [3], through the intersection between two straight lines, one parallel to the initial stiffness and another parallel to the moment-rotation curve post-limit stiffness.

\section{CONCLUSIONS}

The main goal of this investigation was to present a consistent methodology to determine any moment versus rotation curve from experimental tests, including the axial versus bending moment interaction. This methodology can also be applied to results obtained analytically, empirically, mechanically, and numerically. Due to its simplicity and to the fact that its basis is $M-\phi$ curves that already consider the moment versus axial force interaction, it can be easily incorporated into a nonlinear semi-rigid joint finite element formulation. It is also important to observe that the use of the proposed methodology does not change the basic formulation of the non-linear joint finite element, only requiring a rotational stiffness update procedure.

This proposed method is a simple and accurate way of introducing semi-rigid joint experimental test data into structural analysis, through $M-\phi$ curves. Application and validation of the proposed methodology to obtain $M-\phi$ curves, for different axial force levels, were performed against experimental tests executed by Simões da Silva et al. [13] and Guisse et al. [4] on eight flush endplate and on twelve column base joints, respectively.

Finally, it may be suggested that an alternative, though accurate, method to determine $M-\phi$ curves for endplate and baseplate joints, considering the bending moment versus axial force interactions, can be made with a simple linear interpolation between two reference $M-\phi$ curves providing a straightforward procedure to obtain $M-\phi$ curves for any axial force level.

\section{ACKNOWLEGEMENTS}

The authors gratefully acknowledge the financial support provided by the Brazilian National and State Scientific and Technological Developing Agencies: CNPq, CAPES and FAPERJ. This arrangement financed the first author's period as a visiting scholar at Imperial College London.

\section{REFERENCES}

[1] Cerfontaine, F., "Etude de l'interaction Entre Moment de Flexion et Effort Normal Dans Les Assemblages Boulonnés", Doctor en Sciences Appliquées - Université de Liege, Faculté des Sciences Appliquées, 2003. (in French). 
[2] Del Savio, A.A., Andrade, S.A. de, Vellasco, P.C.G.S., Martha, L.F. and Lima, L.R.O. de., "Semi-Rigid Portal Frame Finite Element Modelling Including the Axial Versus Bending Moment Interaction in the Structural Joints", Proceedings of International Colloquia on Stability and Ductility of Steel Structures - SDSS'06, Lisboa, 2006, Vol. 1, pp.1-8.

[3] European Committee for Standardisation, Eurocode 3: Design of Steel Structures - Part 1.8: Design of Joints, Brussels, May 2005.

[4] Guisse, S., Vandegans, D. and Jaspart, J.-P., "Application of the Component Method to Column Bases - Experimentation and Development of a Mechanical Model for Characterization", Report No. MT 195, Liege: Research Centre of the Belgian Metalworking Industry, 1996.

[5] Jaspart J.-P., Braham, M. and Cerfontaine, F., "Strength of Joints Subjected to Combined Action of Bending Moments and Axial Forces", Proceedings of the First European Conference on Steel Structures, Eurosteel, 1999, pp.465-468.

[6] Li, T.Q., Choo, B.S. and Nethercot, D.A., "Connection Element Method for the Analysis of Semi-Rigid Frames", Journal Constructional Steel Research, 1995, Vol. 32, pp.143-171.

[7] Lima, L.R.O. de., "Behaviour of Endplate Beam-to-Column Joints under Bending and Axial Force". Ph.D. Thesis. PUC-Rio, Pontifical Catholic University, Civil Eng. Dept., Rio de Janeiro, Brazil, 2003. (in Portuguese)

[8] Lima, L.R.O. de, Simoes da Silva, L., Vellasco, P.C.G.S. and Andrade, S.A. de., "Experimental Evaluation of Extended End-Plate Beam-to-Column Joints Subjected to Bending and Axial Force", Engineering Structures, 2004, Vol. 26, pp.1333-1347.

[9] Madas, J.P., "Advanced Modelling of Composite Frames Subjected to Earthquake Loading". PhD Thesis, Imperial College Science, Technology and Medicine, University of London, 1993.

[10] Nethercot, D.A. and Zandonini, R., "Methods of Prediction of Joint Behaviour: Beam-to-Column Connections", Structural Connections: Stability and Strength, Elsevier Applied Science, London and New York, Chapter 2, pp.23-62, 1989.

[11] Ramli-Sulong, N.H., "Behaviour of Steel Connections under Fire Conditions". PhD Thesis, Imperial College London, University of London, 2005.

[12] Simões da Silva, L. and Coelho, A.M.G., "An Analytical Evaluation of the Response of Steel Joints under Bending and Axial Force", Computers and Structures, 2001, Vol. 79, pp. 873-881.

[13] Simões da Silva, L., Lima, L.R.O. de, Vellasco, P.C.G.S. and Andrade, S.A. de., "Behaviour of Flush End-Plate Beam-to-Column Joints under Bending and Axial Force", Steel and Composite Structures, 2004, Vol. 4, No. 2, pp. 77-94.

[14] Sokol, Z., Wald, F., Delabre, V., Muzeau, J.-P. and Svarc, M., "Design of Endplate Joints Subject to Moment and Normal Force", Proceedings of Third European Conference on Steel Structures - Eurosteel 2002, Coimbra, Comm Press, 2002, pp. 1219-1228.

[15] Urbonas, K. and Daniunas, A., "Behaviour of Semi-Rigid Steel Beam-to-Beam Joints under Bending and Axial Forces", Journal of Constructional Steel Research, 2006, Vol. 62, pp. 1244-1249.

[16] Vlassis, A.G., Izzuddin, B.A., Elghazouli, A.Y. and Nethercot, D.A., "Design Oriented Approach for Progressive Collapse Assessment of Steel Framed Buildings", Structural Engineering International (Report), SEI Editorial Board, 2006, pp. 129-136.

[17] Wald, F. and Svarc, M., "Experimental with Endplate Joints Subject to Moment and Normal Force. Contribution to Experimental Investigation of Engineering Materials and Structures", CTU Report n: 2-3, Prague, 2001, pp. 1-13.

[18] Wales, M.W. and Rossow, E.C., "Coupled Moment-Axial Force Behaviour in Bolted Joints”, Journal of Structural Engineering, ASCE, 1983, Vol. 109, No. 5, pp. 1250-1266. 


\title{
TOTAL INCREMENTAL ITERATIVE FORCE RECOVERY METHOD AND THE APPLICATION IN PLASTIC HINGE ANALYSIS OF STEEL FRAMES
}

\author{
Fawu Wang ${ }^{1, *}$ and Yaopeng Liu $^{2}$ \\ ${ }^{l}$ Department of Civil Engineering, Nanjing University of Aeronautics and Astronautics, Yudao Street, Nanjing, China \\ ${ }^{2}$ Department of Civil and Structural Engineering, \\ The Hong Kong Polytechnic University, Hunghom, Kowloon, Hong Kong \\ *(Corresponding author: E-mail: fwwang@nuaa.edu.cn)
}

Received: 15 January 2008; Revised: 15 February 2008; Accepted: 20 October 2008

\begin{abstract}
In this paper a total incremental iterative force recovery method suitable to plastic hinge analysis is proposed and it is found that this incremental iteration force recovery procedure has a convergence rate comparable to total secant method. The incremental force recovery manner makes this method be capable of recovering member internal forces and deformations correctly and conveniently in the existence of plastic hinge. The capability of the proposed total incremental iterative force recovery method to cope with plastic hinge is illustrated by two benchmark examples.
\end{abstract}

Keywords: Nonlinear analysis; steel frames; force recovery procedure; plastic hinge

\section{INTRODUCTION}

In the former paper [1] a new total incremental iterative force recovery method is proposed by the authors, which resembles incremental iterative system solution technique and utilizes both the secant and tangent stiffness. It is found through the numerical examples that this incremental iteration force recovery procedure has a convergence rate comparable to total secant iteration method and remains the incremental force recovery manner. This paper provides a detailed description about the application of this new incremental force recovery method in the plastic hinge analysis of framed structures.

Geometric nonlinearity, material plasticity and semi-rigid connections are the most important nonlinear sources of framed structures. When material plasticity is involved, there are mainly two types of methods: (1) plastic zone method; or (2) plastic hinge method. The plastic zone method discretizes each member into many fiber segments longitudinally and transversely and the plastic hinge method assumes plasticity lumped only at specific location along an element the other part of element remains elastic. Although the plastic zone solution may be considered exact, it is not conducive to daily use in engineering design because it is too computationally intensive. On the other hand the refined plastic hinge method can approximate the effects of distributed plasticity along the element length and hinge gradual formation, and remains relatively efficient and economical[2-5].

In practice the incremental and iterative solution strategy is employed to trace the load vs. deflection path of a structure, which can be divided into three stages: the predictor, corrector and error-checking phases. The corrector or force recovery procedure determines the accuracy of the solution while the predictor affects only the convergence speed and direction of iteration [6]. Different solution schemes such as arc-length method can be used to traverse the limit points and capture the post-buckling analysis behavior of the structures [7]. 
The load increment sizes control technique has to be used to inspect the forming of plastic hinge in plastic hinge analysis, which scales back the increment size to the yield surface so that the element forces of specific cross-section comply 'exactly' with those on the yield surface [8]. This technique works for plastic hinge analysis but is tedious and low efficiency as plastic hinge is member based and may occur frequently for complex practical structures. Although a lower limit can be specified to avoid small step length in the case of frequent occurrence of yield hinges, 'exact' scaling to the yield surface is not always possible $[9,10]$. In spite of rapidly increased computed speed and memory capacity of personal computers, the computational efficiency is crucial for the daily use of nonlinear or advanced analysis which requires extensive computational time and effort involving hundreds of load cases and thousands of iterations, not to say when design optimization is used where many design parameters are changed and optimized continuously [11].

Actually in advanced analysis and design the behavior of the whole structure is drawn more attention than the exact time or load step when specific plastic hinge occurs. For the incremental and iterative solution strategy the change in the stiffness of practical complex structures caused by plastic hinge has little difference with that caused by second order effect. The equilibrium path of a structure can be obtained by incremental and iterative solution strategy if the element behavior is correctly modeled no matter in elastic range and plastic range. As the corrector determines the accuracy of the modeling, it is natural and neat to inspect the forming of plastic hinge and recover the correct internal forces and deformations accordingly in force recovery procedure.

In the following of this paper Section 2 summarizes the popular beam-column elements in co-rotational formulation. A simple elastic-plastic hinge model is adopted and section spring is used to model the plastic hinge for consistency in Section 3. The approach of modifying different force recovery procedure for plastic hinge analysis is detailed In Section 4 . The capability of the proposed total incremental iteration force recovery method to cope with plastic hinge is illustrated by two classic numerical examples.

\section{BEAM-COLUMN ELEMENT}

Co-rotational formulation is suitable for the geometrically nonlinear analysis of framed structure as the reference frame of every element can be easily determined by the positions of two nodes of the element. In co-rotational method the displacement $\mathrm{u}$ of element can be decomposed into two parts: the rigid body displacements $\mathrm{u}_{r}$ and the natural deformations $\mathrm{u}_{l}$. The effect of the rigid body displacements $\mathrm{u}_{r}$ is to rotate the initial nodal forces from the last configuration to the present configuration without generation of additional internal forces. The separation of the rigid body displacements from the element deformation makes the formulation of beam-column element simplified as the large deflection and rotation effect can be considered in the deformation extraction operation.

The simplest beam-column element is the cubic element which is robust and successful except for the necessity of using several elements for each member to obtain sufficient accuracy [12]. The stability function element [13] and PEP element [14] are capable of dealing with second-order analysis using one element per member in practical application, which simplifies the analysis and design work as there is no difference between the analysis model and the physical model.

In general the secant stiffness relationships of beam-column element can be expressed as

$$
M_{1 n}=\frac{E I_{n}}{L}\left[\left(c_{1 n}+c_{2 n}\right) \theta_{1 n}+\left(c_{1 n}-c_{2 n}\right) \theta_{2 n}\right]
$$




$$
\begin{aligned}
& M_{2 n}=\frac{E I_{n}}{L}\left[\left(c_{1 n}-c_{2 n}\right) \theta_{1 n}+\left(c_{1 n}+c_{2 n}\right) \theta_{2 n}\right] \\
& P=E A\left[\frac{e}{L}+\sum_{n=x, y} b_{1 n}\left(\theta_{1 n}+\theta_{2 n}\right)^{2}+b_{2 n}\left(\theta_{1 n}-\theta_{2 n}\right)^{2}\right] \\
& M_{x}=\frac{G J+\operatorname{Pr}^{2}}{L} \theta_{x}
\end{aligned}
$$

Where $n=x, y$ are two principal directions of member; $e=$ elongation of the element; $f=\left\{M_{1 \mathrm{y}}, M_{2 \mathrm{y}}\right.$, $\left.M_{1 \mathrm{z}}, M_{2 \mathrm{z}}, P, M_{\mathrm{x}}\right\}^{\mathrm{T}}$ is local internal force vector and $u=\left\{\theta_{1 \mathrm{y}}, \theta_{2 \mathrm{y}}, \theta_{1 \mathrm{z}}, \theta_{2 \mathrm{z}}, e, \theta_{\mathrm{x}}\right\}^{\mathrm{T}}$ is local deformation vector; and $E=$ elasticity modulus; $I_{n}=$ moment of inertia; $A=$ section area; $L=$ length of the element; And $c_{1 \mathrm{n}}, c_{2 \mathrm{n}}$ and $b_{1 \mathrm{n}}, b_{2 \mathrm{n}}$ are axial force relating coefficients and has different forms for different types of element and is not repeated here. It should be noted that the last term of Eq. 3 represents the bowing effect which plays important role in the nonlinear interaction of moments and axial force.

The tangent stiffness matrix can be obtained by taking a variation of the secant stiffness with respect to the displacement degrees of freedom and the axial force by which the second-order effect is considered. And the final expression of the incremental stiffness relationship can be written as

$$
\Delta f=\left[k_{e}\right] \Delta u
$$

Where $\Delta f$ and $\Delta u$ are incremental internal force and deformation vector; $\left[k_{\mathrm{e}}\right]$ is element tangent stiffness matrix. The element tangent stiffness matrix in global coordinates and the global tangent stiffness matrix of the whole structure can be transformed and assembled using the standard procedure of co-rotational formulation and the finite element analysis.

\section{ELASTIC-PLASTIC-HINGE MODEL}

As the main point of this paper is not details of the plastic hinge model, the simple elastic-plastic hinge model is selected to illustrate the application of the proposed force recovery method in plastic hinge analysis. In the elastic-plastic hinge model the cross-section behavior is elastic-perfectly plastic and the plasticity is accounted for by zero-length plastic hinges at two ends of each element.

As the cross-section is assumed to be either ideally elastic or fully plastic, the initial yield surface and fully plastic yield surface are superposed which may be represented by AISC LRFD bilinear interaction equations

$$
\begin{array}{ll}
\frac{P}{P_{y}}+\frac{8}{9} \frac{M}{M_{p}}=1.0 & \text { for } \frac{P}{P_{y}} \geq 0.2 \\
\frac{P}{2 P_{y}}+\frac{M}{M_{p}}=1.0 & \text { for } \frac{P}{P_{y}}<0.2
\end{array}
$$


Where $P_{y}=$ the squash load of the cross section; $M_{p}=$ the plastic moment capacity for member under pure bending action; and $P$ and $M=$ the second-order axial force and bending moment at the cross section being considered.

Although the incremental force-displacement relationships can be deduced for different situations such as plastic hinges at one end and both ends, the section spring concept has the merits of simplicity and consistence. The main idea of the section spring model is the same as semi-rigid spring model that a deformed beam-column element with two springs each attached to the element end node, and in fact the two kinds of springs can be combined as springs in series to simulate semi-rigid connection and plastic hinge together $[15,16]$.

The moment- rotation relationship of the section spring can be written as

$$
\begin{aligned}
& M_{1 n}=k_{1 n}^{s}\left(\theta_{1 n}-\theta_{1 n}^{b}\right)=k_{1 n}^{s} \theta_{1 n}^{s} \\
& M_{2 n}=k_{2 n}^{s}\left(\theta_{2 n}-\theta_{2 n}^{b}\right)=k_{2 n}^{s} \theta_{2 n}^{s}
\end{aligned}
$$

And the spring tangent stiffness can be expressed in the incremental form as

$$
\begin{aligned}
& \Delta M_{1 n}={ }^{t} k_{1 n}^{s}\left(\Delta \theta_{1 n}-\Delta \theta_{1 n}^{b}\right)={ }^{t} k_{1 n}^{s} \Delta \theta_{1 n}^{s} \\
& \Delta M_{2 n}={ }^{t} k_{2 n}^{s}\left(\Delta \theta_{2 n}-\Delta \theta_{2 n}^{b}\right)={ }^{t} k_{2 n}^{s} \Delta \theta_{2 n}^{s}
\end{aligned}
$$

The total and incremental rotational stiffness relationships of beam element can be extracted from the secant stiffness relationship in Eqs. 1 to 4 and the tangent stiffness matrix shown in Eq. 5 respectively

$$
\begin{aligned}
& M_{1 n}=\left(k_{11}^{b}\right)_{n} \theta_{1 n}^{b}+\left(k_{12}^{b}\right)_{n} \theta_{2 n}^{b} \\
& M_{2 n}=\left(k_{21}^{b}\right)_{n} \theta_{1 n}^{b}+\left(k_{22}^{b}\right)_{n} \theta_{2 n}^{b} \\
& \Delta M_{1 n}=\left({ }^{t} k_{11}^{b}\right)_{n} \Delta \theta_{1 n}^{b}+\left({ }^{t} k_{12}^{b}\right)_{n} \Delta \theta_{2 n}^{b} \\
& \Delta M_{2 n}=\left({ }^{t} k_{21}^{b}\right)_{n} \Delta \theta_{1 n}^{b}+\left({ }^{t} k_{22}^{b}\right)_{n} \Delta \theta_{2 n}^{b}
\end{aligned}
$$

Through moment equilibrium condition, the additional internal degree-of-freedoms can be eliminated via the static condensation procedure and finally the total and incremental stiffness relationships can be written as

$$
\begin{aligned}
& {\left[\begin{array}{l}
M_{1 n} \\
M_{2 n}
\end{array}\right]=\frac{1}{\beta_{n}^{\prime}}\left[\begin{array}{cc}
k_{1 n}^{s} \beta_{n}^{\prime}-k_{1 n}^{s} k_{1 n}^{s}\left[\left(k_{22}^{b}\right)_{n}+k_{2 n}^{s}\right] & k_{1 n}^{s} k_{2 n}^{s}\left(k_{21}^{b}\right)_{n} \\
k_{1 n}^{s} k_{2 n}^{s}\left(k_{12}^{b}\right)_{n} & k_{2 n}^{s} \beta_{n}^{\prime}-k_{2 n}^{s} k_{2 n}^{s}\left[\left(k_{11}^{b}\right)_{n}+k_{1 n}^{s}\right.
\end{array}\right]\left[\begin{array}{l}
\theta_{1 n} \\
\theta_{2 n}
\end{array}\right]} \\
& \left.\left[\begin{array}{l}
\Delta M_{1 n} \\
\Delta M_{2 n}
\end{array}\right]=\frac{1}{{ }^{t} \beta^{\prime}}\left[\begin{array}{cc}
k_{1 n}^{s} \beta^{\prime}-k_{1 n}^{s} k_{1 n}^{s}\left[\left(k_{22}^{b}\right)_{n}+k_{2 n}^{s}\right] & k_{1 n}^{s} k_{2 n}^{s}\left(k_{21}^{b}\right)_{n} \\
k_{1 n}^{s} k_{2 n}^{s}\left(k_{12}^{b}\right)_{n} & k_{2 n}^{s} \beta_{n}^{\prime}-k_{2 n}^{s} k_{2 n}^{s}\left[\left(k_{11}^{b}\right)_{n}+k_{1 n}^{s}\right.
\end{array}\right]\right]\left[\begin{array}{l}
\Delta \theta_{1 n} \\
\Delta \theta_{2 n}
\end{array}\right]
\end{aligned}
$$


Where

$$
\begin{aligned}
& \beta_{n}^{\prime}=\left[\left(k_{11}^{b}\right)_{n}+k_{1 n}^{s}\right]\left[\left(k_{22}^{b}\right)_{n}+k_{2 n}^{s}\right]-\left(k_{12}^{b} k_{21}^{b}\right)_{n} \\
& { }^{t} \beta_{n}^{\prime}=\left[\left({ }^{t} k_{11}^{b}\right)_{n}+{ }^{t} k_{1 n}^{s}\right]\left[\left({ }^{t} k_{22}^{b}\right)_{n}+{ }^{t} k_{2 n}^{s}\right]-\left({ }^{t} k_{12}^{b}{ }^{t} k_{21}^{b}\right)_{n}
\end{aligned}
$$

Where left superscript ' $t$ ' represents tangent quantity; $K_{i j}^{b}$ are the relevant stiffness coefficients of stiffness matrix; $K_{\text {in }}^{s}$ are the stiffness of section springs at two ends of element. In computational practice the stiffness of section springs $K^{s}{ }_{i n}$ can be assigned a very large or a very small stiffness according to whether the plastic hinge comes into being. This section spring model can be extended to refined plastic hinge model where the stiffness of the spring is varied from infinity to zero and in this situation the tangent and secant spring stiffness can be different.

\section{FORCE RECOVERY PROCEDURE IN PLASTIC HINGE ANALYSIS}

Before the corrector or force recovery procedure is exercised in the incremental iterative analysis, the element deformation ${ }^{0}[u]$ in last configuration and the present element deformation increments $[\Delta u]$ have been extracted from the system incremental displacements which are computed by the predictor equations. The task of the force recovery procedure is to recover the element internal forces $[f]$ at the current configuration.

\subsection{Total Secant Iterative Method}

The element internal forces can be calculated directly by the secant stiffness in Eqs. 1 to 4 with the present element deformation $[u]$ cumulated from the element deformation ${ }^{0}[u]$ in last configuration and the present deformation increments $[\Delta u]$. The difficulty arises due to the fact that the expression for member axial force as given by Eq. 3 involves bowing functions which in turn are functions of the axial force parameter $q=P L^{2} / E I$. As a result the whole equations are implicit functions of axial force $P$. To solve this problem, an axial force iteration procedure has to be adopted which is based on first-order Taylor series expansion of Eq. 3.

As the secant stiffness equations are explicit and exact, this force recovery method has the capability of good and rapid convergence than the force incremental method in elastic range. If plastic hinge behavior is considered, there is abrupt point in the element force-deformation curve when plastic hinge comes into being. The explicit secant stiffness relationship does exist in simple situation but has different forms for elastic and plastic range. The situation is further complicated as axial force iteration procedure must be adopted to obtain the exact axial force value which is the basis of determining the exact position of yield surface. So this force recovery method is recommended only for the elastic nonlinear analysis of frames.

\subsection{Force Incremental Method}

The element recovery forces can also be cumulated from the force increments which can be calculated by the product of element natural stiffness and natural deformations

$$
\Delta f=k_{e} \Delta u
$$


in which $k_{\mathrm{e}}$ is the element natural stiffness matrix. The internal forces of the element can be updated by adding the incremental element forces to the element internal forces in last configuration

$$
f={ }^{0} f+\Delta f
$$

The accuracy of force increments is only approached in the first order of the displacement increments so the load increment step should be small enough to obtain a sufficiently accurate solution.

The secant iterative method has obstacle to cope with the abrupt point in plastic hinge analysis. In incremental type force recovery methods things get simple and natural: firstly scale back to the right position where plastic hinge occurs, then calculate the force increment causing by deformation increment remained using the modified incremental stiffness. The whole procedure can be illustrated as follows

$$
\begin{aligned}
& { }^{e} f=f-\alpha \Delta f \\
& \Delta f^{\prime}=k_{e}^{\prime} \Delta u \\
& { }^{p} f={ }^{e} f+\alpha \Delta f^{\prime} \\
& \alpha=\frac{M-M_{p}}{\Delta M}
\end{aligned}
$$

Where ${ }^{e} f$ and ${ }^{p} f$ are element internal forces in elastic and plastic range respectively; $k_{e}$ ' is the modified incremental stiffness after plastic hinge formed and $\alpha$ is a scale factor that reverse the force to the right position where plastic hinge occurs. When the moment axial force interaction equations such as Eqs. 6-7 are introduced as yield surface, a one dimensional search procedure should be adopted to find the exact scale factor instead of Eq. 25 being used directly.

Actually the force-deformation relationship is not a straight line but a curve with abrupt point as illustrated by Figure 1. Because of the tangent approximation, the force increment method can only get force-deformation path OA'B', which has the equal internal forces with the real path OAB but the deformation where plastic hinge occurs is different. This is not a problem as the load cycle step should be small enough in force incremental method and the introduced error can be neglected.

\subsection{Total Incremental Iterative Method}

The secant iterative method is more accurate and rapid while the force incremental method has the advantage of simplicity and flexibility of coping with abrupt point on force-deformation curve. Their combined use is a sensible choice in improving the computational accuracy and efficiency, just like the incremental-iterative method used in the solution of system equilibrium equations. Simulating the modified Newton-Raphson method, the total incremental iteration can be written as

$$
\begin{gathered}
{ }^{i}[\Delta f]={ }^{i}[k][\Delta u] \\
{ }^{i}[f]={ }^{i-1}[f]+{ }^{i}[\Delta f] \\
{ }^{i}[u]={ }^{i-1}[u]+{ }^{i}[\Delta u]
\end{gathered}
$$




$$
{ }^{i+1}[\Delta u]={ }^{0}[u]+\sum_{k=1}^{i}{ }^{k}[\Delta u]-{ }^{i}[u]
$$

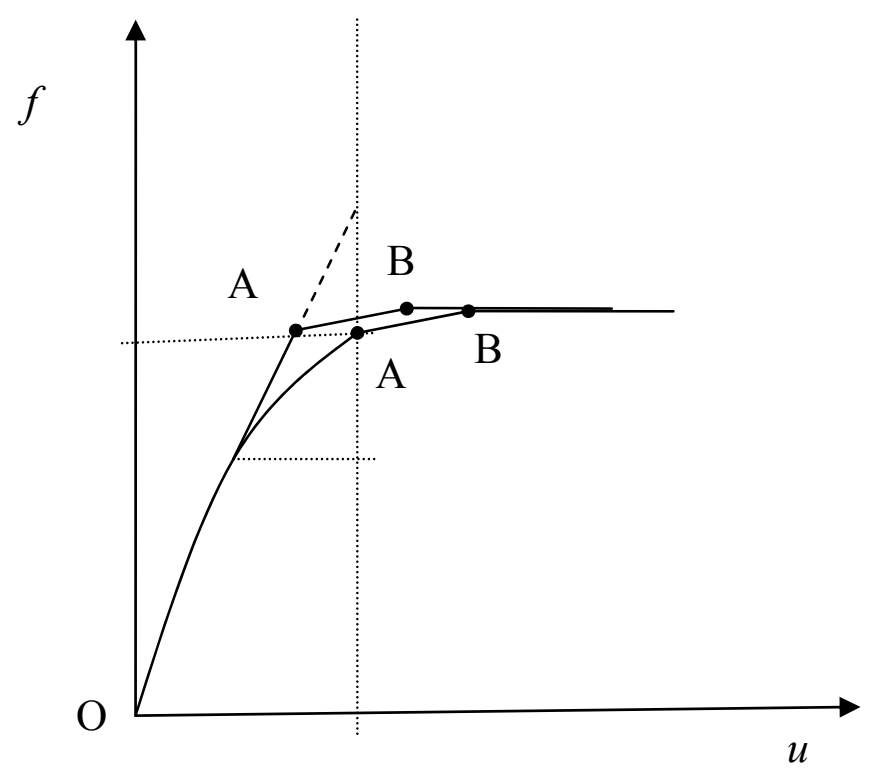

Figure 1. Illustration of Different Force Recovery Method

In this iteration the total deformation ${ }^{\mathrm{i}}[u]$ can be easily calculated by the reverse form of secant stiffness Eqs. 1-4. This iterative process of Eqs. 26-29 continues until the unbalanced deformation ${ }^{\mathrm{i}}[\Delta u]$ is sufficiently small. And it should be noted that the tangent matrix in Eq. 26 is kept unchanged during the iteration, which makes this iteration use minimal computational effort compared with system equation solution.

The same as the force incremental method, a one dimensional search procedure should be adopted to scale member state right back to the yield surface and the exact scale factor can be obtained. Unlike the force incremental method, the exact point A that plastic hinge occurs can be converged by the iteration procedure. If the explicit secant stiffness relationships with plastic hinge exist, another iteration as Eqs. 26-29 can be continued to arrive the specified deformation state. Otherwise Eq. 24 can be utilized in the case of only incremental force-deformation can be obtained conveniently when refined plastic hinge model is used. As the force recovery method can simulate the member exactly in the existence of plastic hinge, especial load increment sizes control technique to scale the whole structure back to plastic hinge point is not needed.

\section{NUMERICAL EXAMPLES}

\subsection{Vogel Portal Frame}

Figure 2 shows a portal frame proposed and solved by Vogel. The frame possesses an out-of-plumbness of 1/400 and a member initial imperfection of 1/1000 along the column length. The section of the beam is HEA-340 and that of columns is HEB-300. This paper uses a single element and elastic plastic hinge model with the proposed force recovery method to model the beam and the column. The computed ultimate load factor is 1.00 which is close to the result of [17]. Load-displacement curves by different methods are showed in Figure 3. The total incremental iterative method and force incremental method give almost same results, but the total incremental 
iterative method can still obtain approximate collapse load even with 10 load cycles. It is viewed from numerical iteration process that the total incremental iterative method has nearly same performance in elastic and plastic range, with 2 or 3 more iterations increasing in presence of plastic hinge.

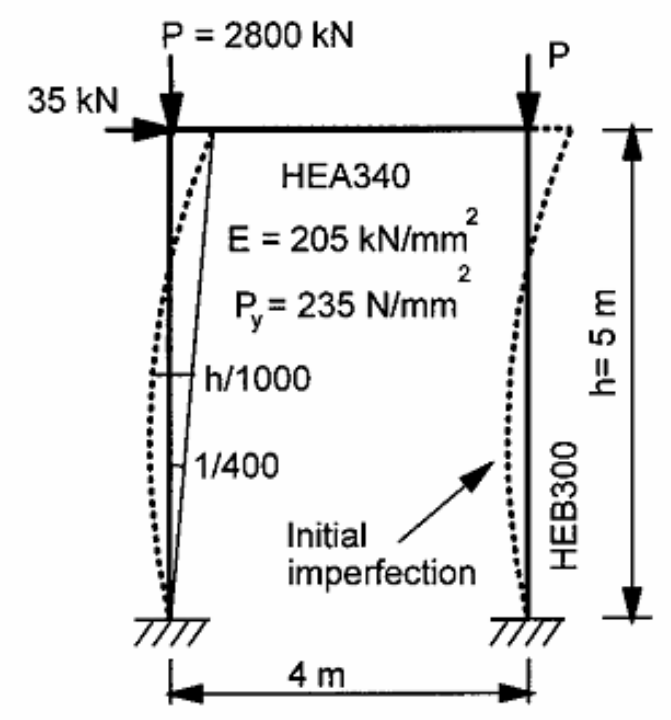

Figure 2. Vogel Portal Frame

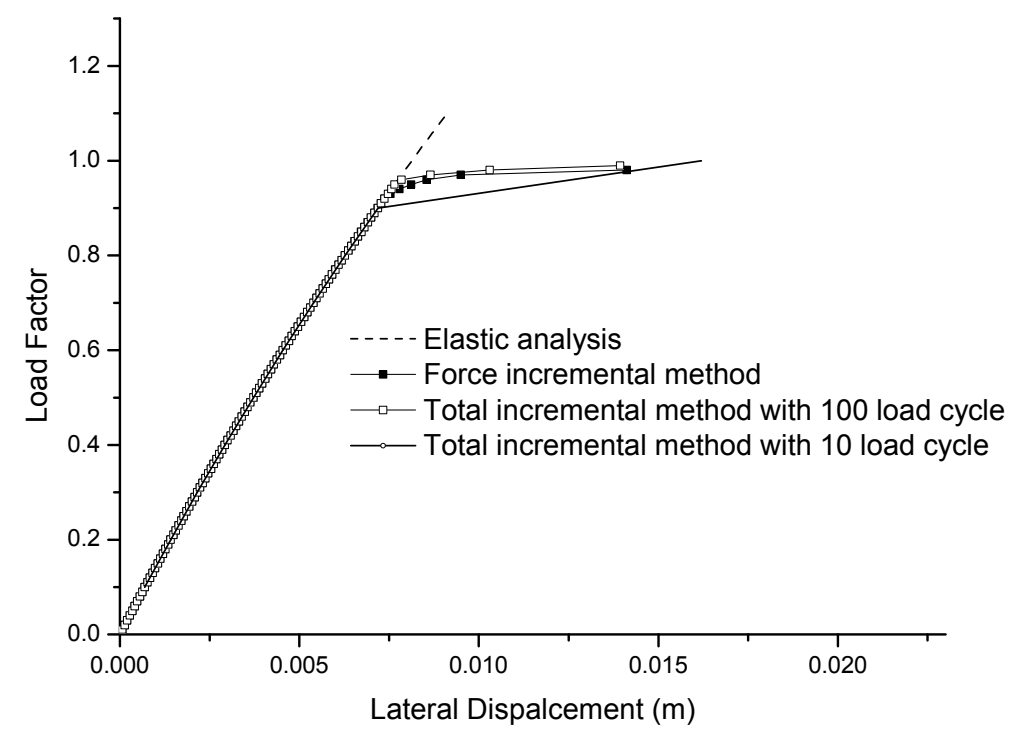

Figure 3. Load-displacement Curve of Vogel Portal Frame

\section{$5.2 \quad$ Vogel Six Story Frame}

Figure 4 shows the well known Vogel six story frame. The frame possesses an out-of-plumbness of $1 / 400$ and a member initial imperfection of $1 / 1000$ along the column length. The section of the beam is IPE-240 to IPE-400 and that of columns is HEB-200 to HEB-260. This paper uses a single element and elastic plastic hinge model with the proposed force recovery method to model the beam and the column. The computed ultimate load factor is 1.1 which is which is close to the result of [17]. From Figure 5 it can be seen that almost same collapse load is obtained by the total incremental iterative method with different load steps. As the exact load path is not always needed in design, the proposed method is effective and rapid, which is demanded and welcomed by engineers. Figure 6 shows the bending moment diagram and plastic hinge position of Vogel six story frame near collapse. 


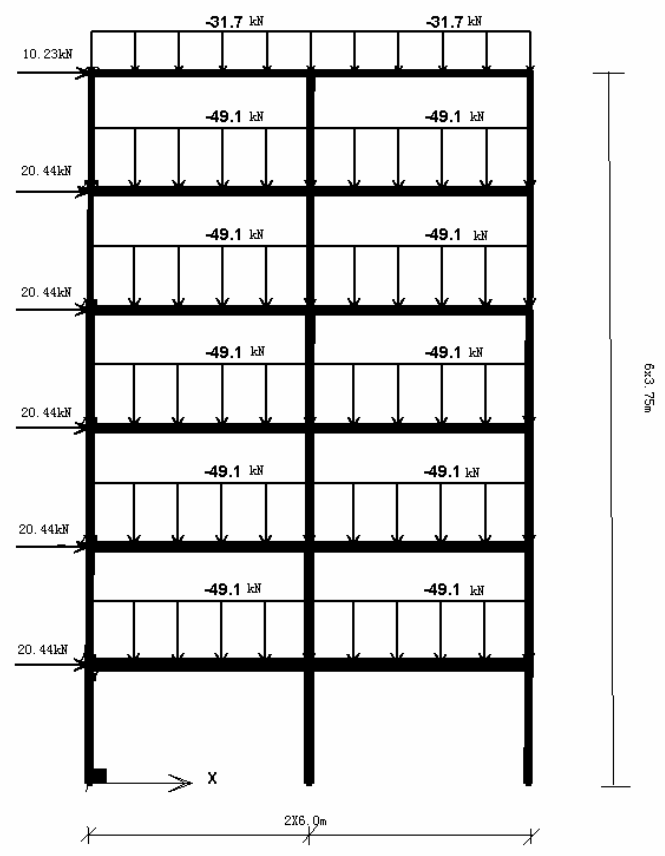

Figure 4. Vogel Six Story frame

\section{CONCLUSION}

This paper provides a detailed description about the application of the total incremental iterative force recovery method in the plastic hinge analysis of framed structures. This method utilizes both the secant and tangent stiffness and remains incremental manner in the process of recovering internal forces, which is convenient to the modeling of plastic hinge. As the force recovery method can simulate the member exactly in the existence of plastic hinge, especial load increment sizes control technique to scale the whole structure back to plastic hinge point is not needed. Numerical examples show this method is useful in the plastic hinge analysis and daily design of steel frames.

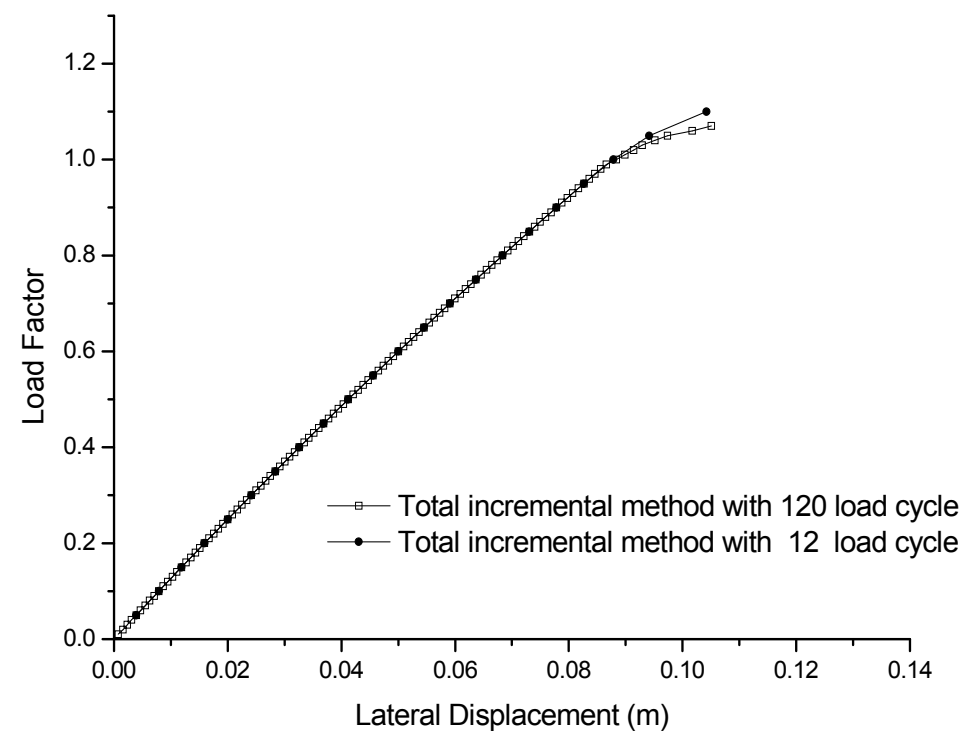

Figure 5. Load-displacement Curve of Vogel Six Story Frame 


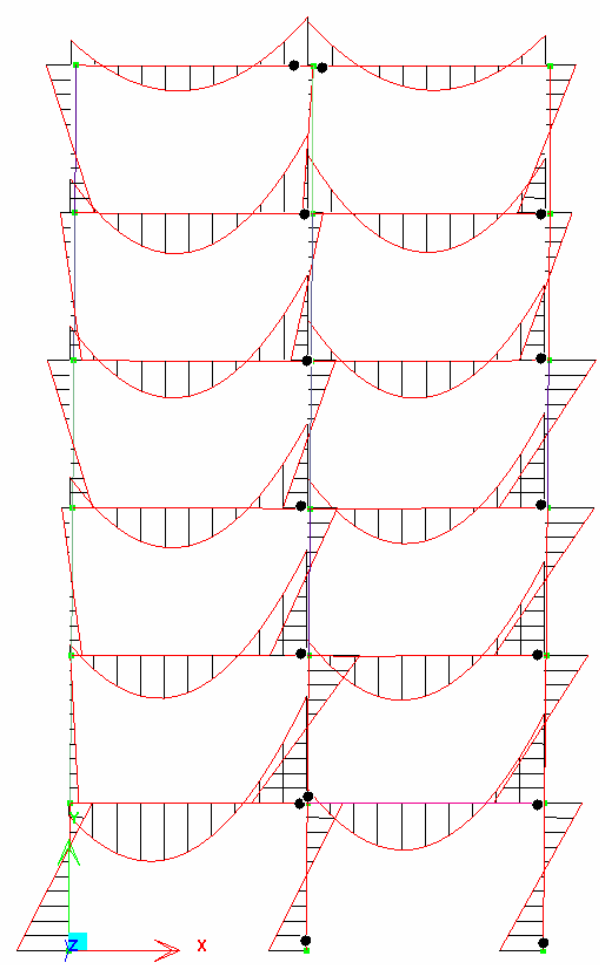

Figure 6. Bending Moment Diagram and Plastic Hinge Position of Vogel Six Story Frame

\section{REFERENCES}

[1] Wang, F.W. and Liu, Y.P., "Total and Incremental Iteration Force Recovery Procedure for the Nonlinear Analysis of Framed Structures", Advanced Steel Construction, 2009, Vol. 5, pp.500-514.

[2] Liew, J.Y.R., White, D.W. and Chen, W.F., "Second-order Refined Plastic-hinge Analysis for Frame Design. Part I", Journal of Structural Engineering, ASCE, 1993, Vol. 119, pp. 3196-3216.

[3] Liew, J.Y.R., White, D.W. and Chen W.F., "Second-order Refined Plastic-hinge Analysis for Frame Design. Part II", Journal of Structural Engineering, ASCE, 1993, Vol. 119, pp. 3217-3237.

[4] Zhou, Z.H. and Chan, S.L., "Elastoplastic and Large Deflection Analysis of Steel Frames by One Element Per Member. I: One Hinge Along Member", Journal of Structural Engineering, ASCE, 2004, Vol. 130, pp. 538-544.

[5] Chan, S.L. and Zhou, Z.H., "Elastoplastic and Large Deflection Analysis of Steel Frames by One Element Per Member. II: Three Hinges Along Member", Journal of Structural Engineering, ASCE, 2004, Vol. 130, pp. 545-553.

[6] Yang, Y.B., Lin, S.P. and Leu, L.J., "Solution Strategy and Rigid Element for Nonlinear Analysis of Elastically Structures Based on Updated Lagrangian formulation", Engineering Structures, 2007, Vol. 29, pp. 1189-1200.

[7] Chan, S.L. and Chui, P.P.T., "Non-linear Static and Cyclic Analysis of Steel Frames with Semi-rigid Connections", Elsevier Science Ltd., 2000.

[8] Chen, H., "Nonlinear Inelastic Analysis of Steel-concrete Composite Frames.” PhD thesis, Dept. of Civil Engineering, National Univ. of Singapore, Singapore, 2000.

[9] Liew, J. Y. R., Chen, H., Shanmugam, N.E. and Chen, W.F., "Improved Nonlinear Plastic Hinge Analysis of Space Frame Structures", Engineering Structures, 2000, Vol. 22, No.. 10, pp. 1324-1338. 
[10] Liew, J.Y.R., and Tang, L.K., "Advanced Plastic Hinge Analysis For the Design of Tubular Space Frames", Engineering Structures, 2000, Vol. 22, No. 7, pp. 769-783.

[11] Wang, F.W. and Chan, S.L., "Optimization and Sensitivity Analysis of Space Frames Allowing for Large Deflection”, Engineering Structures, 2006, Vol. 28, pp. 1395-1406.

[12] Chan, S.L. and Kitipornchai, S., "Geometric Nonlinear Analysis of Asymmetric Thin-walled Beam-columns", Engineering Structures, 1987, Vol.9, pp. 243-254.

[13] Gu, J.X., "Large Displacement Elastic Analysis of Space Frames Allowing for Flexural-torsional Buckling of Beams", Dept. of Civil and Structural Engineering, Hong Kong Polytechnic University, Hong Kong, 2004.

[14] Chan, S.L. and Zhou, Z.H., "Pointwise Equilibrating Polynomial Element for Nonlinear Analysis of Frames", Journal of Structural Engineering, 1994, Vol. 120, No. 6, pp. 1703-1717.

[15] Iu, C.K. and Chan, S.L., "A Simulation-based Large Deflection and Inelastic Analysis of Steel Frames under Fire". Journal of Constructional Steel Research, 2004, Vol. 60, pp. 1495-524.

[16] Iu, C.K., "Inelastic Finite Element Analysis of Composite Beam on the Basis of Plastic Hinge Approach", Engineering Structures, 2008, Vol. 30, pp. 291-302.

[17] Toma, S. and Chen, W.F., "European Calibration Frames for Second-order Inelastic Analysis", Engineering Structures, 1992, Vol. 14, No. 1, pp. 7-14. 


\title{
EXPERIMENTAL STUDY OF EXTERNAL DIAPHRAGM JOINT CONNECTING CHS COLUMN AND H-SHAPED BEAM
}

\author{
W.Q. Li ${ }^{1, *}$, Y.Y. Chen ${ }^{1,2}$, W. Wang ${ }^{1,2}$, Y.J. Xu ${ }^{3}$ and X.D. Lv $^{3}$ \\ ${ }^{1}$ Department of Structural Engineering, Tongji University, Shanghai 200092, China \\ ${ }^{2}$ State Key Laboratory for Disaster Reduction in Civil Engineering, Shanghai 200092, China \\ ${ }^{3}$ China Northwest Building Design Research Institute, Xi'an 710003, China \\ *(Corresponding author: E-mail: lwqxyf631@hotmail.com)
}

Received: 25 February 2008; Revised: 13 October 2008; Accepted: 20 November 2008

\begin{abstract}
This paper focuses on external diaphragm joint connecting CHS column and H-shaped beam. Quasi-static tests on three cruciform specimens and finite element analysis using PATRAN/MARC were carried out. The width of diaphragm plate is taken as the test parameter. Four types of failure mode were observed: local buckling of CHS column, noticeable shear deformation at joint zone, doglegged deformation of CHS column, as well as crack at the heat affected zone of CHS column. The specimens exhibited full hysteresis loops, but different initial stiffness and ultimate capacity. Analytical results match the test well and are used to comprehend the force transfer mechanisms between beam flange and diaphragm plate.
\end{abstract}

Keywords: External Diaphragm Joint, CHS Column, H-shaped Beam, Quasi-static test, finite element analysis

\section{INTRODUCTION}

For steel building frames, circular hollow section tube and H-steel are suitable for column and beam members respectively. Basically, there are three types of joints connecting CHS column and $\mathrm{H}$-shaped beams commonly used in moment-resisting steel frames: through diaphragm joint, inner diaphragm joint and external diaphragm joint. Among them, external diaphragm joint is most convenient for construction. When forming the external diaphragm joint, the diaphragm plates are welded around the periphery of the column to avoid the cut of the tube in joint zone. It should be potential benefit considering a water-cooling system in steel column to be used (Sakumoto [1]) in order to prevent from fire heating in high-rise buildings.

Though studies have been carried out by previous research, there are still some unclear points remained for the behavior of the joint. Rink et al. [2] investigated the static strength of external diaphragm joint by numerical method. They found that increasing the width of diaphragm plate would not increase the ultimate capacity as much as that predicted by the AIJ formula. Kamba and Kanatani [3] explained the AIJ formula in detail and concluded that difference of failure mechanism and limiting width-to-thickness ratio of diaphragm plate resulted in the discrepancy between results of Rink et al. and AIJ formula. On the other hand, in the engineering practices in China, the width of the external diaphragm not less than $70 \%$ of the width of the beam flange is required. It is on the assumption that the force from the beam flange is transferred entirely through the external diaphragm. Wang et al. [4] performed finite element analysis of external diaphragm type joint under vertical load and pointed out the Chinese provision may be conservative or dangerous under different ratios of axially compressive load to axially yielding force of the column section.

In this paper, the authors report experimental research about the beam-column joint in which the width of external diaphragm plate is taken as a parameter and the specimen is under combined force condition including compressive load on column and cyclic shear-moment action on ends of members. Additionally, finite element analysis using PATRAN/MARC was conducted, and the force transfer mechanism between beam flange and diaphragm plate was discussed. 


\section{EXPERIMENTAL PROGRAM}

A beam-column connection between the inflection points of a frame subjected to lateral loads can be idealized as shown in Figure 1 (a). The lateral load can be directly obtained by applying horizontal load to the column top. The same deformation of the connection can be achieved by modeling the connection as shown in Figure 1 (b) and the lateral load is simulated by applying antisymmetric loads at the beam ends. The second way is adopted to develop the testing system for convenience.

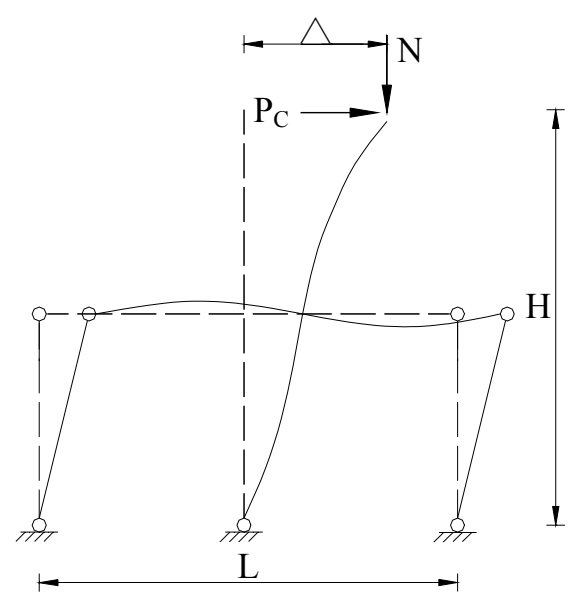

(a) Direct Modeling

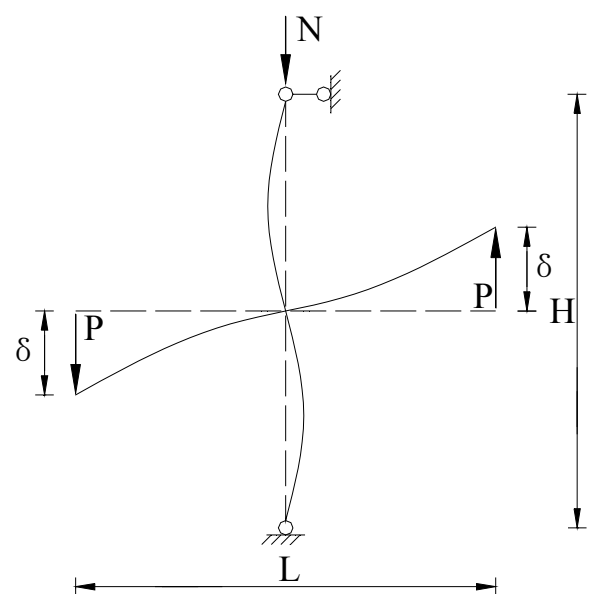

(b) Indirect Modeling

Figure 1. Modeling of Beam-Column Connection

\subsection{Test Specimen}

A total of three specimens were tested in this study which consisted of two H-300 $\times 175 \times 12 \times 8 \mathrm{~mm}$ beams connected to a $350 \times 9 \mathrm{~mm}$ CHS column by means of diaphragm plates. The test variable is the width of diaphragm plate. Specimen details are shown in Figure 2 and geometric parameters for the three specimens are listed in Table 1 , where $d_{c}, t_{c}$ refer to the diameter and wall thickness of the CHS column, $b_{f}, t_{f}$ to the width and thickness of the beam flange, $h_{b}, t_{w}$ to the height of the beam and the thickness of its web, and $h_{s}, t_{s}$ to the width and thickness of the external diaphragm. The radius of the auxiliary circle marked in dashed line in Figure 2 is the sum of the radius of the column and the width of external diaphragm plate. $\mathrm{R}$ is the radius of the circle tangent to the auxiliary circle and the edges of the orthogonal beam flanges and can be evaluated by Eq. 1. The size of the specimen DIA-125 was selected to be one-half scale corresponding to its prototype structure. The height of the column, $\mathrm{H}$, is $3025 \mathrm{~mm}$ and the total beam length, L, between the two loading points at cantilever ends is $3600 \mathrm{~mm}$. The material properties of the specimens were measured by conducting coupon tests and the average yield stress of the column, beam flange and external diaphragm was found to be $464 \mathrm{MPa}, 389 \mathrm{MPa}$ and 420MPa respectively. 


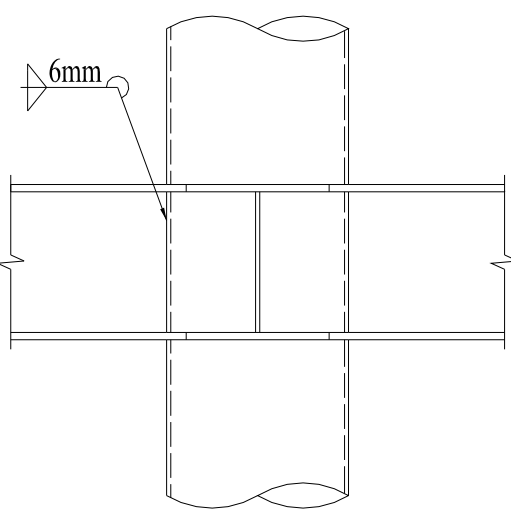

(a) Elevation View

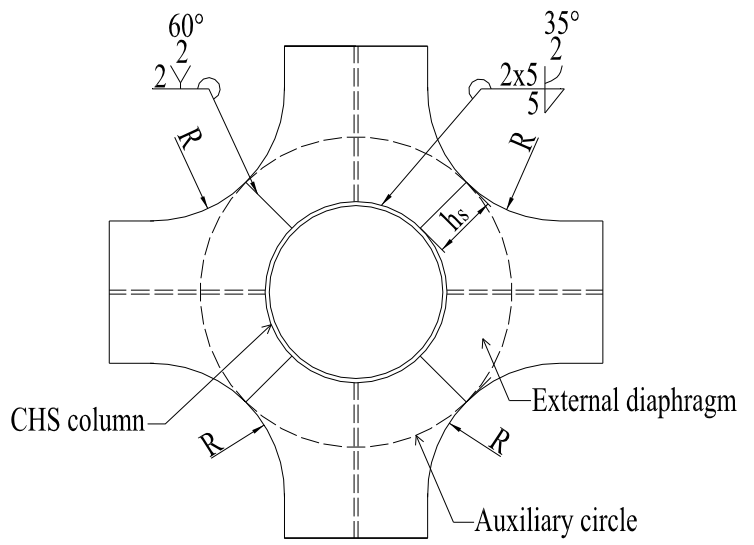

(b) Plan View

Figure 2. Specimen Details

Table 1. Geometric Parameters of Test Specimens

\begin{tabular}{|c|c|c|c|c|c|c|c|c|c|}
\hline Specimen & $\begin{array}{c}\mathrm{d}_{\mathrm{c}} \\
(\mathrm{mm})\end{array}$ & $\begin{array}{c}\mathrm{t}_{\mathrm{c}} \\
(\mathrm{mm})\end{array}$ & $\begin{array}{c}\mathrm{b}_{\mathrm{f}} \\
(\mathrm{mm})\end{array}$ & $\begin{array}{c}\mathrm{t}_{\mathrm{f}} \\
(\mathrm{mm})\end{array}$ & $\begin{array}{c}\mathrm{h}_{\mathrm{b}} \\
(\mathrm{mm})\end{array}$ & $\begin{array}{c}t_{\mathrm{w}} \\
(\mathrm{mm})\end{array}$ & $\begin{array}{c}\mathrm{h}_{\mathrm{s}} \\
(\mathrm{mm})\end{array}$ & $\begin{array}{c}\mathrm{t}_{\mathrm{s}} \\
(\mathrm{mm})\end{array}$ & $\mathrm{h}_{\mathrm{s}} / \mathrm{b}_{\mathrm{f}}$ \\
\hline DIA- $125^{*}$ & \multirow{3}{*}{350} & \multirow{3}{*}{9} & \multirow{3}{*}{175} & \multirow{3}{*}{12} & \multirow{3}{*}{300} & \multirow{3}{*}{8} & 125 & 14 & 0.71 \\
\hline DIA-75 & & & & & & & 75 & 14 & 0.43 \\
\hline DIA-45 & & & & & & & 45 & 14 & 0.26 \\
\hline
\end{tabular}

*The beam flange is locally widened to $275 \mathrm{~mm}$ at the end connected to the external diaphragm.

$$
R=\operatorname{ctg}\left(22.5^{\circ}\right) \cdot\left(\frac{d_{\mathrm{c}}}{2}+h_{\mathrm{s}}-\frac{\sqrt{2}}{2} b_{\mathrm{f}}\right)
$$

\subsection{Test Setup}

The specimens were tested in State Key Laboratory of Disaster Prevention in Civil Engineering at Tongji University. The test setup was designed to implement the load and boundary conditions shown in Figure 1 (b). Figure 3 shows a schematic of the test setup. Spherical bearings were placed at the two ends of the beam as well as the top and the bottom of the column to achieve the expected restraint as perfect pin-boundary conditions. The spherical bearing at the top of the column was attached to the steel reaction frame with one tubular brace, and the other at the bottom of the column was fixed on the foundation, which was attached to the steel reaction frame with a foundation beam. The spherical bearings at the two ends of the beam were connected with the two-way hydraulic jacks by high-strength bolts. A load cell was mounted between the two-way jack and the spherical bearing at the east beam end to monitor the actual applied load. These jacks were fixed on the foundation beam and the steel reaction frame respectively. A $3200 \mathrm{kN}$ one-way hydraulic jack was placed at the top of the column to apply compressive load. Lateral reaction trusses assembled from angle irons were provided to hold back out-of-plane movement of the beams. The test setup had a displacement capacity of $\pm 250 \mathrm{~mm}$ and a load capacity of $\pm 300 \mathrm{kN}$.

The specimens were instrumented to monitor the response during the loading process in order to comprehend the specimen behavior, as shown in Figure 4. Strain gauges were glued at the external diaphragm, the panel zone, the beam and the column near the joint zone to investigate local response. Displacement transducers were placed at the west and east end of the beam, the top and the bottom of the column. Corresponding to those positions, displacement measurements are denoted by $\delta_{1}, \delta_{2}, \delta_{3}$ and $\delta_{4}$. The relative displacement of the west and east beam end, $\delta$, was calculated from the total displacement by subtracting the rigid body motion of the specimen, as 
shown in Eq. 2 and Eq. 3. Readings from the displacement transducers at the panel zone were used to calculate the panel shear deformation $\gamma$. The vertical load, P, applied to the ends of cantilever beams was measured by the load cell. The global rotation of the connection, $\theta$, and the moment of the connection, M, can be computed by Eq. 4 and Eq. 5, respectively.

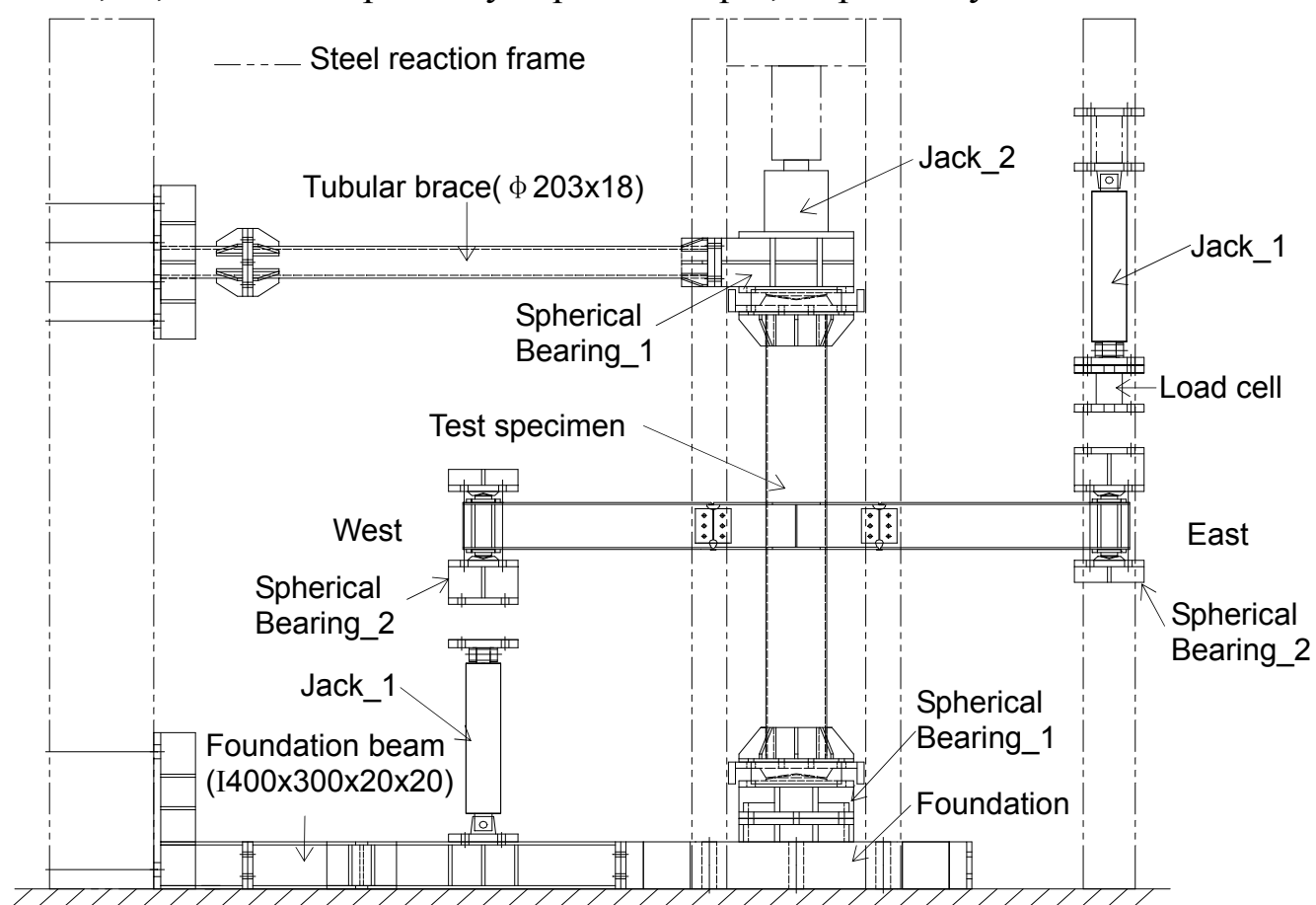

(Lateral reaction trusses are not shown for clarity)

Figure 3. Schematic of Test Setup

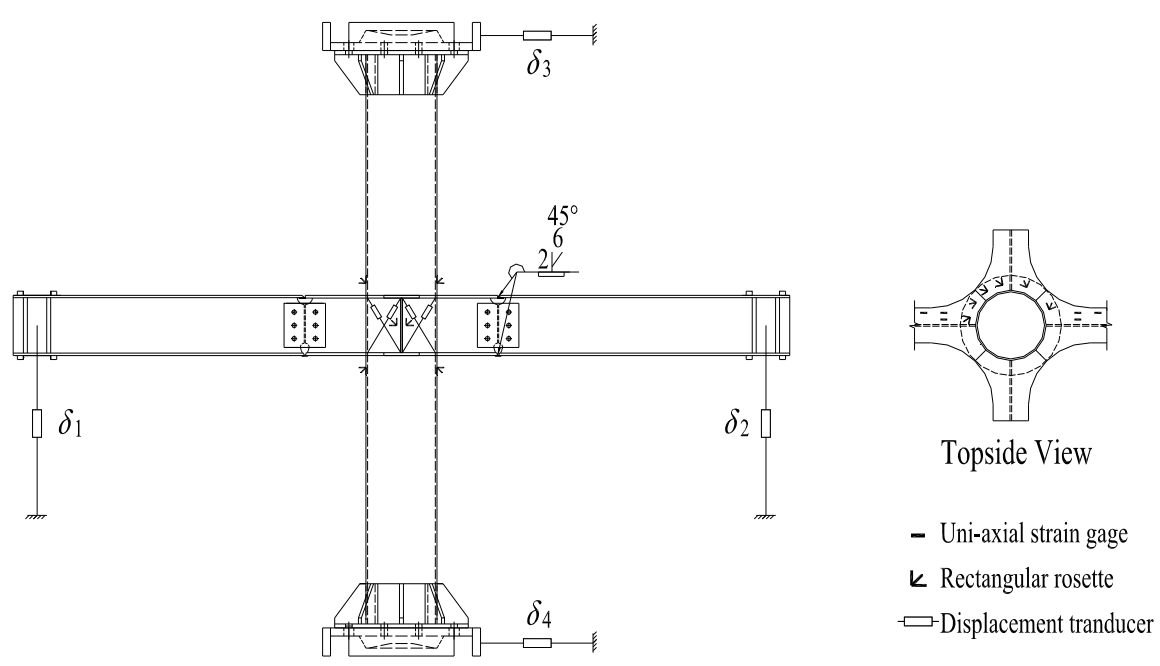

Figure 4. Specimen Instrumentation

$$
\begin{aligned}
& \delta=\delta_{1}+0.5 L \cdot\left(\delta_{3}-\delta_{4}\right) / H \\
& \delta=\delta_{2}-0.5 L \cdot\left(\delta_{3}-\delta_{4}\right) / H \\
& \theta=\delta /\left(0.5 L-0.5 d_{\mathrm{c}}\right) \\
& M=P \cdot\left(0.5 L-0.5 d_{\mathrm{c}}\right)
\end{aligned}
$$




\subsection{Test Procedure}

Each test specimen was subjected to a constant axial load on column and cyclic loads on beams. Axially compressive load was first applied to the top of the column by the compressive hydraulic jack until it reached the given value $0.27 \mathrm{~N}_{\mathrm{y}}$, where $\mathrm{N}_{\mathrm{y}}$ is the axially yielding force of the member section, and held constant during the entire loading process. Antisymmetric cyclic loads were then synchronously applied to cantilever ends of beams by the two-way hydraulic jacks. The specimens were tested under force control at the beginning cycles. After yielding, the test was converted to displacement control, following a loading history composed of stepwise increasing deformation cycles and cycling continued until the ultimate capacity of the specimen was reached or the apparatus limitation was exceeded. In fact, displacement control can not be automatically realized by the two-way jacks and must be mated with manually monitoring the readings from the displacement transducers arranged at beam ends.

\section{EXPERIMENTAL RESULTS}

\subsection{Hysteretic Behavior}

The plots of the connection moment normalized by the complete plastic moment capacity of beam versus the global rotation of the connection are shown in Figure 5 to 7 . The curves indicate good capacity of energy dissipation for the three specimens by the full and stable hysteretic loops. The maximum moment capacity of specimen DIA-125 and DIA-75 are slightly greater than or almost same as the full plastic moment of its beams, while that of specimen DIA-45 is around 10\% lower than the beam full plastic moment.

The initial stiffness and maximum moment capacity of the specimens are shown in Table 2 . The initial stiffness, $\mathrm{Ki}$, is obtained by linear regression analysis of the $\mathrm{M}-\theta$ history from the first elastic cycle. The maximum moment capacity, $\mathrm{M}_{\max }$, is calculated by multiplying the maximum force recorded by the load cell by the distance from the center of the two-way jack to the interface of the column.

The maximum moment capacity of specimen DIA-125 is $8 \%$ higher than that of specimen DIA-75 and $25 \%$ higher than that of specimen DIA-45. The initial stiffness of specimen DIA-125 is $16 \%$ higher than that of specimen DIA-75 and 22\% higher than that of specimen DIA- 45 . The fact indicates that the initial stiffness and maximum moment capacity of the specimen are closely related to the width of diaphragm plate.

\subsection{Failure Mode}

Specimens after tests are shown in Figure 8 to 10. Local buckling occurred at the column near the external diaphragm for all the three specimens when the connection moment reached $293 \mathrm{kN}-\mathrm{m}$, $268 \mathrm{kN}-\mathrm{m}$ and $258 \mathrm{kN}-\mathrm{m}$, respectively. Typical strain development in the corresponding zone is shown in Figure 11. For specimen DIA-125, the panel zone underwent noticeable shear deformation and crack was observed in the heat affected zone of the column near the external diaphragm. For specimen DIA-75, the doglegged deformation of CHS column appeared in the large deformation range. For specimen DIA-45, noticeable shear deformation of the panel zone was also observed. Local buckling or fracture of the diaphragm plate did not appear.

The strain gauge data show that the specimens differed in yielding sequence of different locations. For specimen DIA-125, the diaphragm yielded after the panel zone and the column. For specimen 
DIA-75, the diaphragm yielded following the yielding of panel zone while ahead of the column. For specimen DIA-45, the diaphragm yielded before the panel zone and the column.

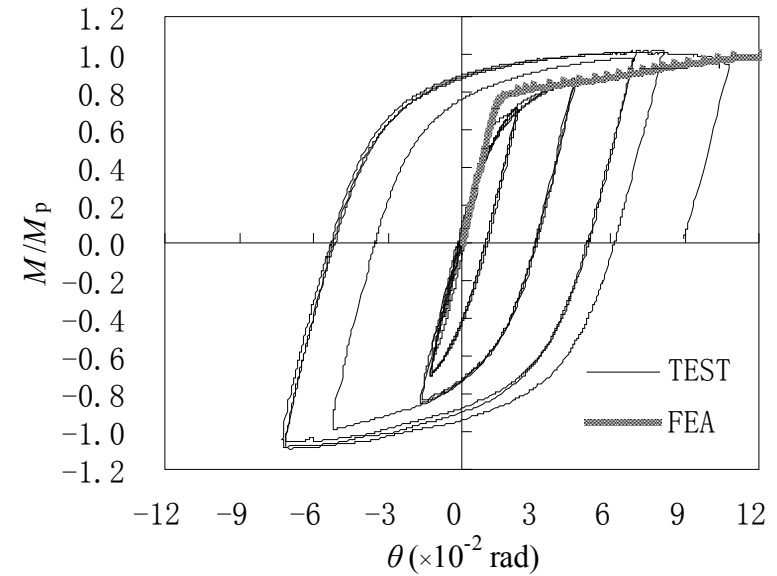

(a) Beam West End

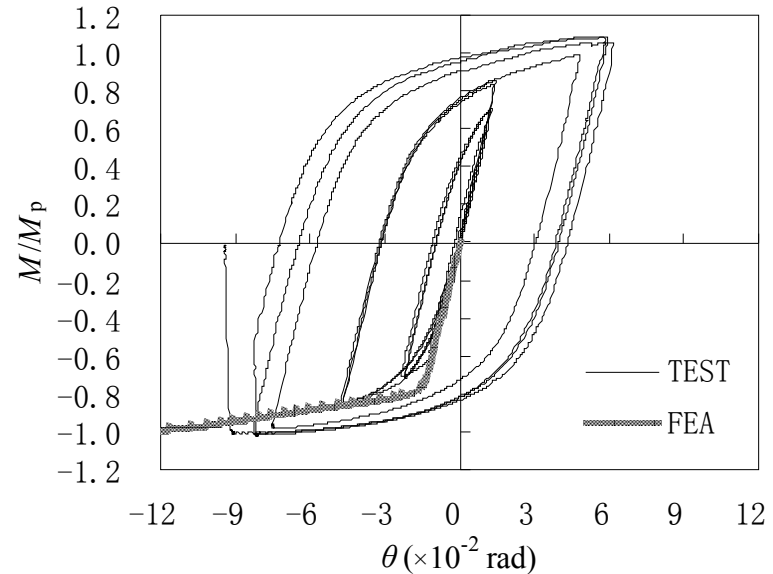

(b) Beam East End

Figure 5. Moment-Rotation Hysteretic Loops for Specimen DIA-125

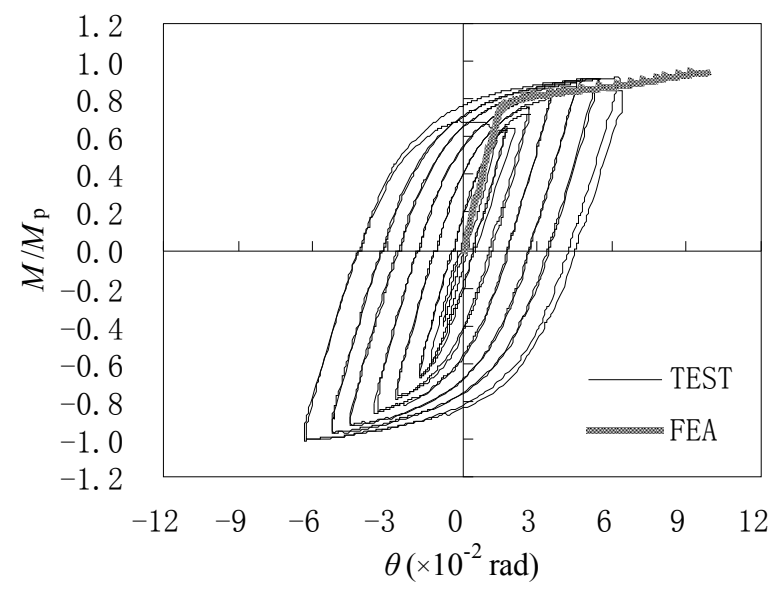

(a) Beam West End

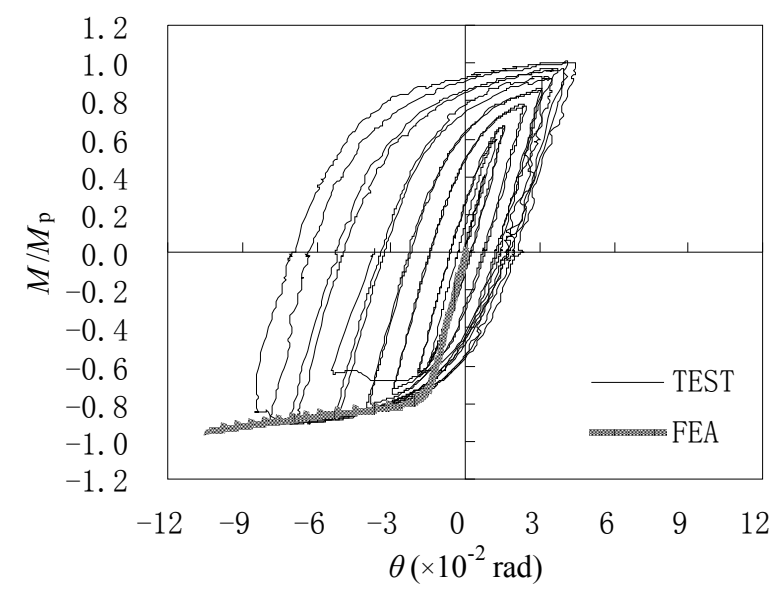

(b) Beam East End

Figure 6. Moment-Rotation Hysteretic Loops for Specimen DIA-75

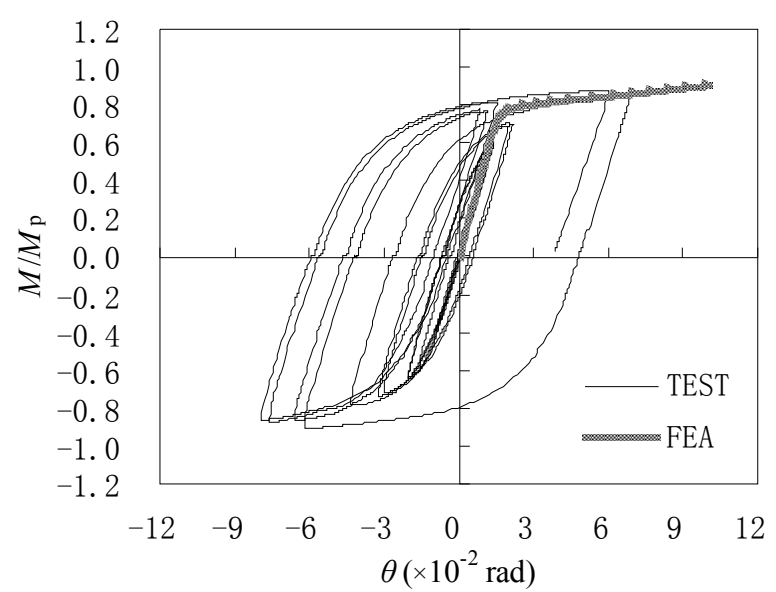

(a) Beam West End

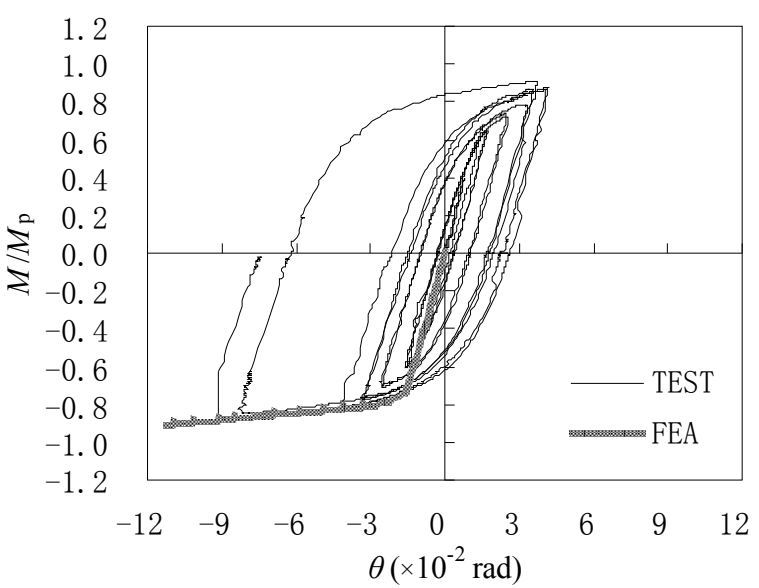

(b) Beam East End

Figure 7. Moment-Rotation Hysteretic Loops for Specimen DIA-45 
Table 2. Test Results

\begin{tabular}{c|c|c}
\hline Specimen & $\begin{array}{c}K_{\mathrm{i}} \\
(\mathrm{kN}-\mathrm{m} / \mathrm{rad})\end{array}$ & $\begin{array}{c}M_{\max } \\
(\mathrm{kN}-\mathrm{m})\end{array}$ \\
\hline DIA-125 & 16968 & 322 \\
\hline DIA-75 & 14574 & 297 \\
\hline DIA-45 & 13887 & 258 \\
\hline
\end{tabular}

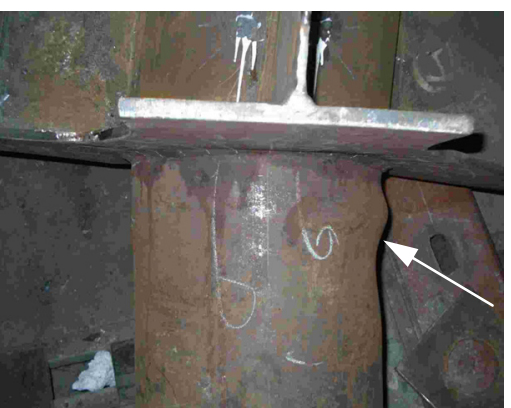

(a) Local Buckling

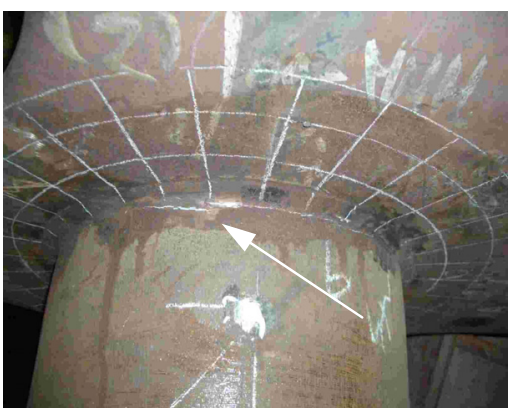

(b) Crack

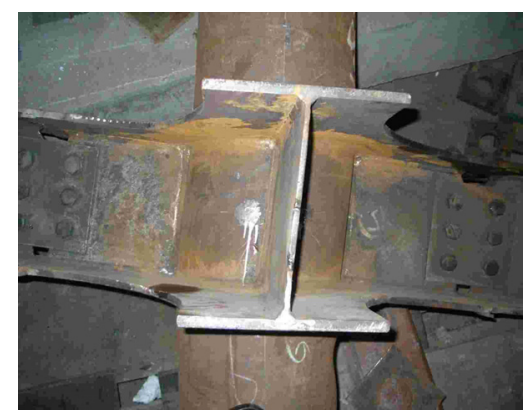

(c) Excessive Shear Deformation

Figure 8. Failure Mode of Specimen DIA-125

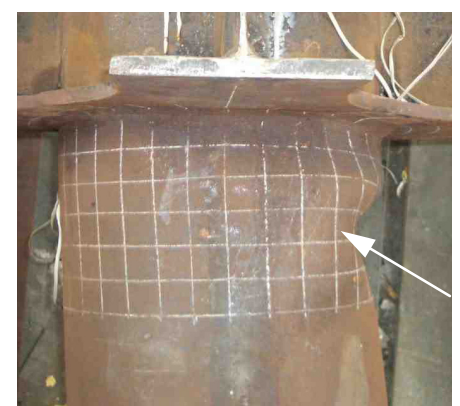

(a) Local Buckling

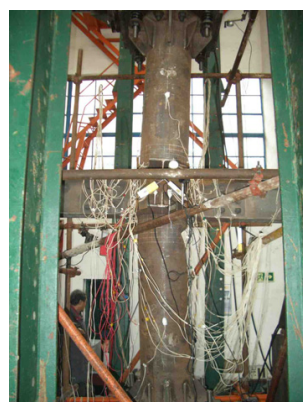

(b) Doglegged Deformation

Figure 9. Failure Mode of Specimen DIA-175

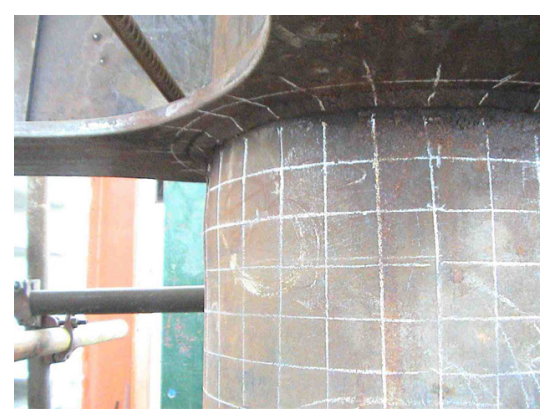

(a) Local Buckling

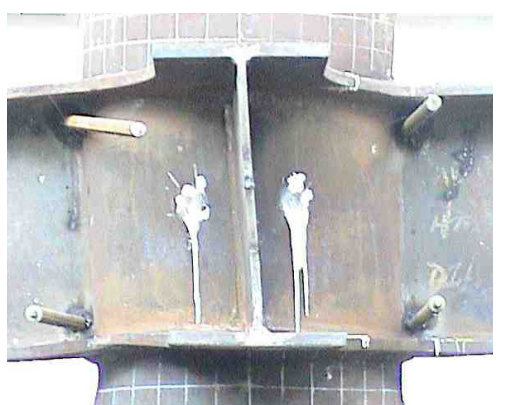

(b) Excessive Shear Deformation

Figure 10. Failure Mode of Specimen DIA-45 


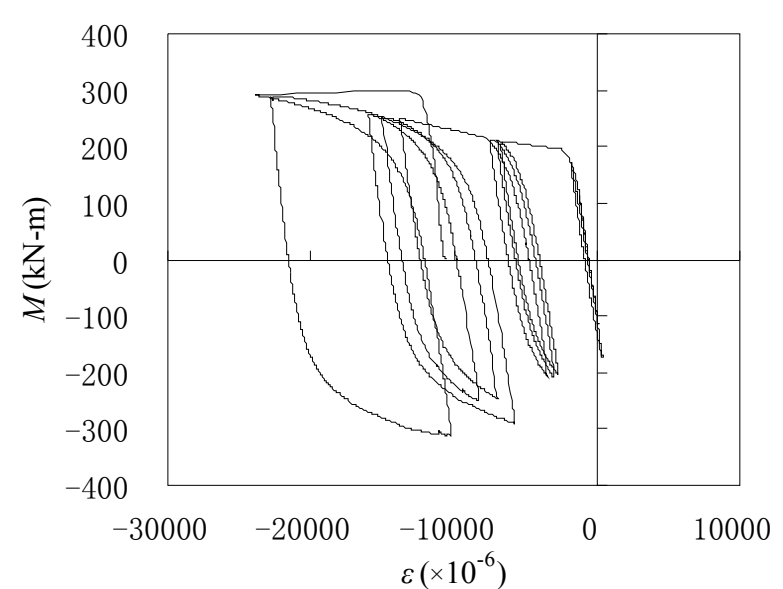

(a) DIA-125

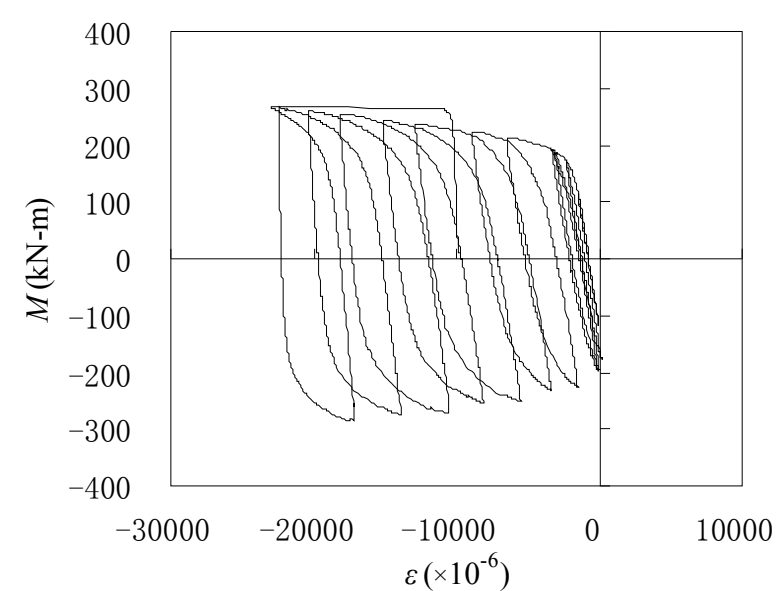

(b) DIA-75

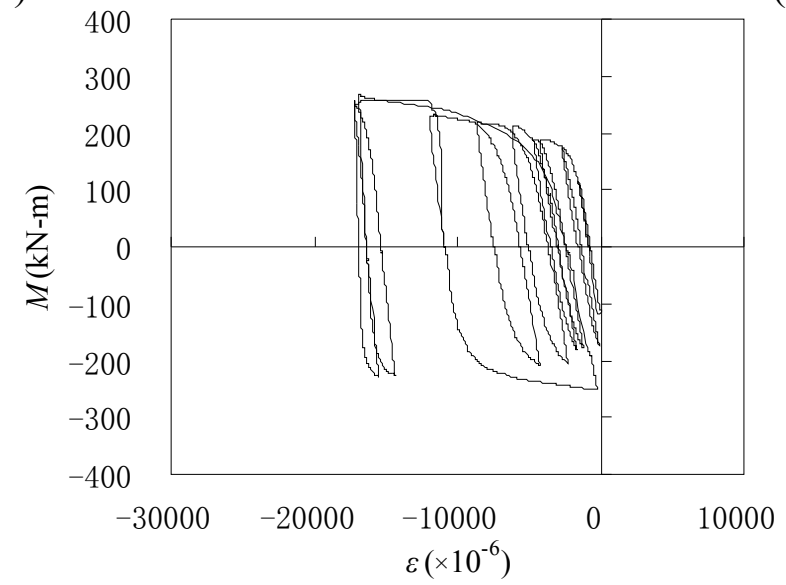

(c) DIA-45

Figure 11. Typical Strain Development in Local Buckling Zone

\subsection{Comparison with AIJ Formula}

The yield shear strength of the joint panel and the connection resistance can be calculated according to Recommendation of Architectural Institute of Japan [5, 6], and the results are listed in Table 3. In the table, $\mathrm{M}_{\mathrm{sy}}, \mathrm{M}_{\mathrm{cy}}, \mathrm{M}_{\mathrm{y}}$ and $\mathrm{M}_{\mathrm{u}}$ are the calculated moment capacity of connection corresponding to the yield shear strength of the panel zone, the yield moment strength of the column, the yield strength of the connection and the ultimate strength of the connection respectively. The yielding sequences of different parts of the three specimens predicted by design formulas are in accordance with those observed in the tests as mentioned above. However, the maximum strength of the specimen obtained from the tests does not correspond well with the ultimate strength of the connection predicted by the AIJ formula.

Table 3. Calculated Strength of Different Parts

\begin{tabular}{c|c|c|c|c}
\hline Specimen & $\begin{array}{c}M_{\text {sy }} \\
(\mathrm{kN}-\mathrm{m})\end{array}$ & $\begin{array}{c}M_{\mathrm{cy}} \\
(\mathrm{kN}-\mathrm{m})\end{array}$ & $\begin{array}{c}M_{\mathrm{y}} \\
(\mathrm{kN}-\mathrm{m})\end{array}$ & $\begin{array}{c}M_{\mathrm{u}} \\
(\mathrm{kN}-\mathrm{m})\end{array}$ \\
\cline { 4 - 5 } DIA-125 & \multirow{2}{*}{200} & \multirow{2}{*}{260} & 294 & 392 \\
\cline { 1 - 3 } \cline { 4 - 5 } DIA-75 & & 233 & 310 \\
\cline { 1 - 3 } & & & 172 & 229 \\
\hline
\end{tabular}

*Geometric parameters of the connections are slightly beyond the valid range suggested by AIJ 


\section{FINITE ELEMENT ANALYSIS}

\subsection{Finite Element Modeling}

Monotonic finite element analysis is performed to comprehend the force transfer mechanism between beam flange and external diaphragm. PATRAN and MARC [7] are used for mesh generation and analysis, respectively. Four-node quadrilateral shell elements with reduced integration (MRAC element type 140) are used to model the specimens. The mesh at the joint panel and external plates is densified. A representative finite element model is shown in Figure 12. Material properties are input according to the data from coupon tests. Classical metal plasticity theory based on Von Mises yield criterion, isotropic hardening and associated flow rule is assumed. Actual load and boundary conditions are specified. Geometric nonlinearities are accounted for through a small-strain, large-displacement formulation.

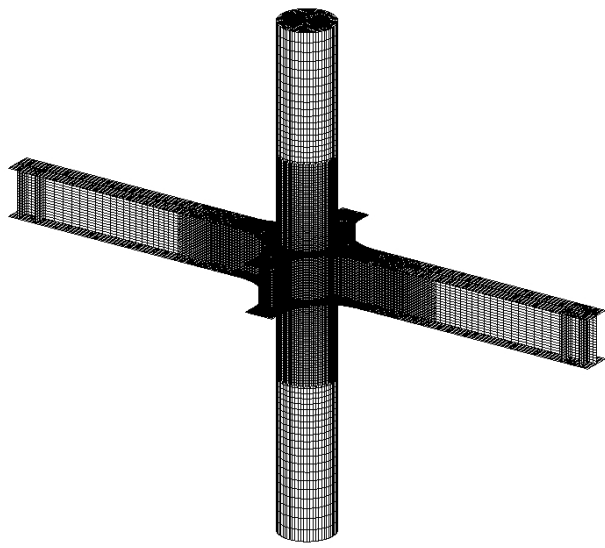

(a) Specimen

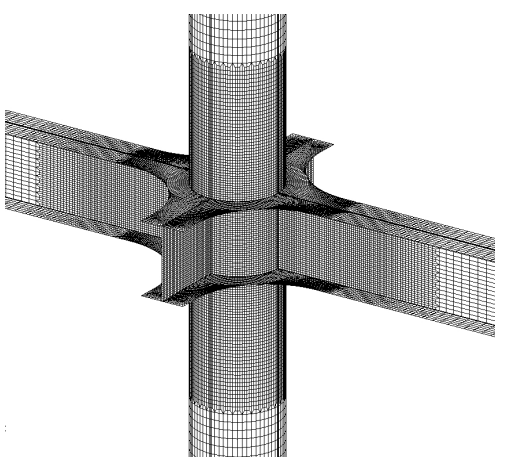

(b) Joint

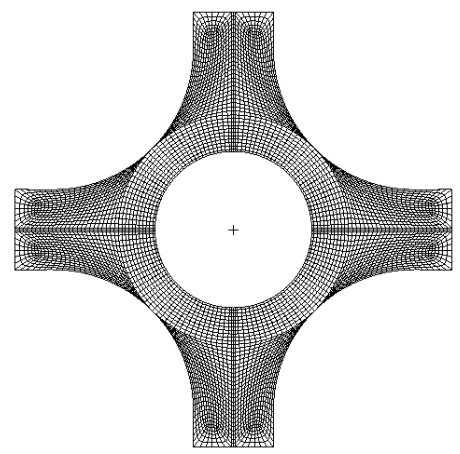

(c) External Diaphragm

Figure 12. Typical Finite Element Mesh

\subsection{Verification}

The numerical moment-rotation curves are shown in Figure 5 to 7 . One of the typical deformed shapes predicted by numerical analysis is exhibited in Figure 13. Although no direct comparison was possible, good correlation between the numerical and the experimental work was obtained.

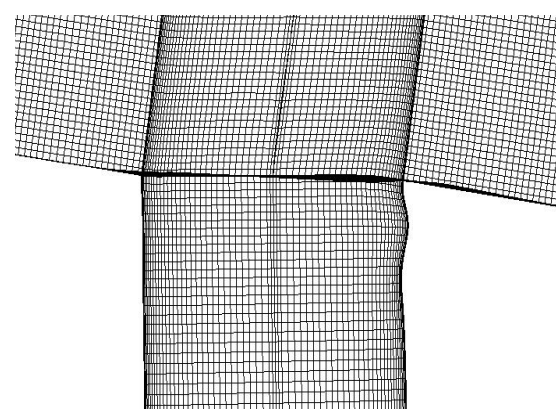

Figure 13. Deformed Shape of FE Model (DIA-125)

\subsection{Discussion of Force Transfer Mechanism}

As mentioned above, in the engineering practices in China, the width of external diaphragm plate is only related to the width of beam flange and stipulated as: 


$$
h_{\mathrm{s}} \geq 0.7 b_{\mathrm{f}}
$$

As compared to AIJ formula, Chinese provision is simple and may be easier to comprehend. It is deduced on the assumption that the force $C_{f}\left(T_{f}\right)$ from the beam flange is transferred entirely through the external diaphragm, as shown in Figure 14.

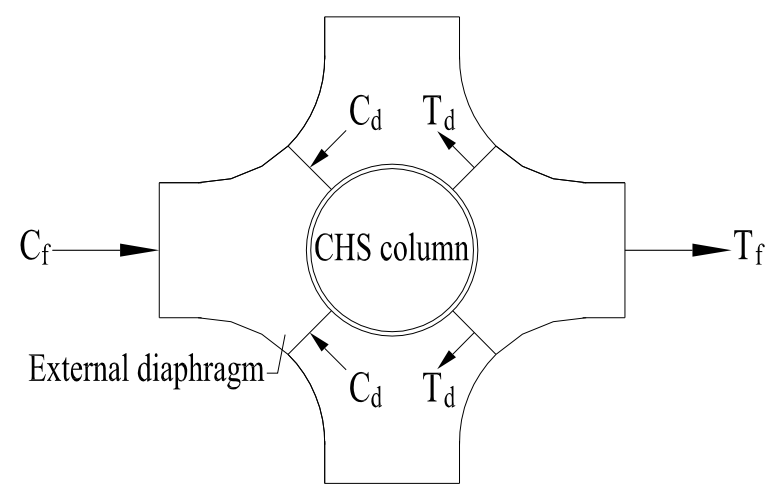

Figure 14. Equilibrium of Horizontal Forces Based on Chinese Provision

Referring to Figure 14, the following equilibrium expressions can be observed.

$T_{\mathrm{f}}=\sqrt{2} T_{\mathrm{d}}$

Where $T_{d}$ is the axial force born by the diaphragm plate. If the thickness of the diaphragm plate is equal to that of beam flange and the diaphragm plate is required to yield after the beam flange, Eq. 6 must be satisfied.

Finite element analyses show that the horizontal forces in the beam flanges are transferred to both the external plate and the column. Table 4 gives the contributions of the diaphragm plate and the column to transferring the beam flange forces at elastic range, where $C_{f}$ and $T_{f}$ refer to the flange compressive and tensile force, $\beta_{\mathrm{d}}$ and $\beta_{\mathrm{c}}$ to the contribution of the diaphragm plate and the column. $\beta_{\mathrm{d}}$ drops with $h_{\mathrm{s}}$ decreasing and $\beta_{\mathrm{d}}$ is smaller under flange compressive force than under tensile force.

Based on the numerical analysis, it can be seen that Chinese provision may be conservative for diaphragm plate. However, as the force transferred to the column may be strong enough to result in the plastic failure of tube wall, Chinese provision can be improved by taken into geometric size of the column.

Table 4. Contribution of different parts to transferring flange force

\begin{tabular}{|c|c|c|c|c|}
\hline \multirow[b]{2}{*}{ Specimen } & \multicolumn{2}{|c|}{$T_{\mathrm{f}}$} & \multicolumn{2}{|c|}{$C_{\mathrm{f}}$} \\
\hline & $\begin{array}{c}\beta_{\mathrm{d}} \\
(\%)\end{array}$ & $\begin{array}{c}\beta_{\mathrm{c}} \\
(\%)\end{array}$ & $\begin{array}{c}\beta_{\mathrm{d}} \\
(\%)\end{array}$ & $\begin{array}{c}\beta_{\mathrm{c}} \\
(\%)\end{array}$ \\
\hline D & 85 & 15 & 78 & 22 \\
\hline DIA-7 & 71 & 29 & 63 & 37 \\
\hline DIA-45 & 58 & 42 & 51 & 49 \\
\hline
\end{tabular}




\section{CONCLUSIONS}

The cyclic behavior of external diaphragm joint connecting CHS column and H-shaped beam is presented. The beam flange force transfer mechanism is discussed through finite element analysis. Based on the experimental and numerical study, the following conclusion can be drawn:

(1) The specimens have excellent capacity of energy dissipation. External diaphragm joint is efficient.

(2) The width of external diaphragm plate influences on the initial stiffness and ultimate moment capacity of the specimens.

(3) Test results do not correspond well with AIJ formula.

(4) Chinese provision about the width of external diaphragm plate maybe conservative. However, it needs to be improved to preclude plastic failure of the tube wall.

\section{ACKNOWLEDGEMENTS}

The presented work was supported by the State Key Laboratory for Disaster Reduction in Civil Engineering of China. Any opinions, findings, conclusions, and recommendations expressed in this paper are those of the writers and do not necessarily reflect the views of the sponsors. Technical helps from China Northwest Building Design Research Institute are greatly appreciated.

\section{REFERENCES}

[1] Sakumoto, Y., "New Materials and Methods for Steel Frame Fire Resistance", Shokoku Publication, Tokyo, Japan, 1994.

[2] Rink, H.D., de Winkel, G.D., Wardenier, J. and Puthli, R.S., "Numerical Investigation into the Static Strength of Stiffened I Beam-to-Column Connections", Proc. of 4th Int. Symp. on Tubular Structures, Delft, Netherlands, 1991, pp. 461-470.

[3] Kamba, T. and Kanatani, H., "Design Formulae for CHS Column-to-Beam Connections with Exterior Diaphragms", Proc. of 5th Int. Symp. on Tubular Structures, Nottingham, U.K., 1993, pp. 249-256.

[4] Wang, L.W., Feng, Y. and Huang, Z.Y., "Investigation About the Joints of the Tube Column and Beam on the Steel Frame Structure", Building Structure, 2005, Vol. 6, No. 35, pp.32-34.

[5] AIJ, "Recommendations for Design of Connections in Steel Structures", Architectural Institute of Japan, Tokyo, Japan, 2001.

[6] AIJ, "Recommendations for the Design and Fabrication of Tubular Truss Structures in Steel”, Architectural Institute of Japan, Tokyo, Japan, 2002.

[7] Chen, H.H. and Qi, P., "Training Tutorial and Examples for MSC. Patran/Marc", Science Press, Beijing, China, 2004. 


\title{
DEVELOPMENT OF AN ENGINEERING METHODOLOGY FOR THERMAL ANALYSIS OF PROTECTED STRUCTURAL MEMBERS IN FIRE
}

\author{
Hong Liang, Stephen Welch* and José L. Torero \\ BRE Centre for Fire Safety Engineering, University of Edinburgh, \\ King's Buildings, Edinburgh, EH9 3JL, United Kingdom \\ *(Corresponding author: E-mail: s.welch@ed.ac.uk)
}

Received: 25 February 2008; Revised: 6 March 2009; Accepted: 11 March 2009

\begin{abstract}
A novel CFD-based methodology for thermal analysis of protected steelwork in fire has been developed to overcome the limitations of existing methodologies. This is a generalised quasi-3D approach with computation of a "steel temperature field" parameter in each computational cell. It accommodates both uncertainties in the input parameters and possible variants to the specification by means of many simultaneous thermal calculations. A framework for the inclusion of temperature/time-dependent thermal properties, including the effects of moisture and intumescence, has been established. The method has been implemented as the GeniSTELA submodel within SOFIE RANS CFD code. The model is validated with respect to the BRE large compartment fire tests. Sensitivity studies reveal the expected strong dependencies on certain properties of thermal protection materials. The computational requirements are addressed to confirm the practicability of the tool in simultaneously running a large number of parametric variants. Ultimately, the steel temperature field prediction provided by GeniSTELA provides far more flexibility in assessing the thermal response of structures to fire than has been available hitherto; hence it could be further used for the structural response analysis, demonstrating the potential practical use of the method to improve the efficiency and safety of the relevant structural fire safety design.
\end{abstract}

Keywords: CFD; thermal analysis; quasi-3D; GeniSTELA; SOFIE

\section{INTRODUCTION}

Increasing interest in assessing the performance of structures in fire is driving the development of an array of modelling methodologies to be used in fire safety engineering design. Whilst traditionally most code-based design has been based on simple calculations, referencing measured fire performance in standard tests, the progressive shift towards performance-based design has opened the door to use of advanced methods based on numerical models. These approaches will not replace standard testing, but they can already be used in a complementary fashion, to extend the application of test data.

Some simplified modelling methods have also been established, such as the protected member equation in Eurocode 3 (EC3) [1], but as with all semi-empirical methods the results will tend to be conservative and there are of necessity a number of simplifying assumptions. CFD-based methodologies can in principle provide a much more detailed description of the thermal environment and the effects of localised heating, which could be used in conjunction with thermal analysis models to examine structural performance.

There are currently various approaches used to link the CFD-based models and the thermal analysis models. For example a range of advanced methodologies have been developed to couple the CFD model with the thermal and structural model, such as those examined in the FIRESTRUC [2] project with code pairs ANSYS-CFX, FDS-ANSYS, VESTA-STELA etc. All these implementations have certain aspects of pros and cons. 
Prior to this work [3], a dedicated fine-mesh thermal modelling tool, known as STELA (Solid ThErmaL Analysis), has been implemented within the RANS CFD code SOFIE [4]. However, this research suggested that detailed thermal analysis of structural members in the context of simulations of full-scale building fires remains problematic. This is partly due to the difference of scale between the mesh which can be afforded for the fire and that required for the thermal analysis of the structure, a particular problem with structured meshes, and also the generally high computational demands for coupled analyses. Moreover, existing approaches, no matter how the thermal model is implemented into the CFD model, either in embedded manner within the CFD code such as STELA, or by more generalised couplings such as with FDS-ANSYS, VESTA-DIANA, are limited to a specific structural arrangement of interest since it is necessary to define all model parameters in advance. Simulations must be repeated if details such as the structural geometry or the thermal properties are changed, a very inefficient procedure.

A more general and flexible methodology has now been proposed, still within the context of a CFD fire simulation, as reported elsewhere $[5,6]$. This is based on computation of a set of "steel temperature field" parameters within the whole of the calculation domain, accommodating, by means of simultaneous calculations, both uncertainties in the input parameters and possible variants to the specification. Hence the need for repeat simulations is bypassed. Furthermore, by predicting the "virtual member" temperatures at each point in space the limitations of existing methods with regards to the position of the structural component are overcome. Considering the potentially great computational costs associated with the large numbers of thermal analysis calculations required (equal to the number of gas-phase cells times the number of variants studied in the simultaneous calculations), approximate methods are employed to reduce the full 3D thermal response problem down to treatments which are essentially $1 \mathrm{D}$ but which include appropriate representations of the heat transfer processes in the other dimensions to reconstruct a quasi-3D solution. The further development, in particular, the development of a modelling representation for the effects of intumescent performance in fire is also described here. This new generalised methodology has been implemented as a submodel within the SOFIE RANS CFD code and is known as GeniSTELA (Generalised Solid ThErmaL Analysis).

\section{METHODOLOGY DEVELOPMENT}

\subsection{Brief Description}

When protected steelwork is exposed to fire, heat is transferred to the structure through a layer of insulation. The transient heating response of the member can in principle be described using conventional methods based on numerical heat transfer. However, full 3D analyses impose great computational demands, due to the large numbers of cells required in order to adequately resolve the steep thermal gradients during the initial heating. Even if the computational resource is available, in simple deterministic models there is no direct mechanism to accommodate uncertainties in the thermal properties and member specification. To overcome these problems, with an appropriate balance between accuracy and tractability, a novel quasi-3D analysis methodology has been developed [5]. This is achieved by constructing a generalised 1D model and further considering the $2 \mathrm{D}$ or $3 \mathrm{D}$ effects within the heat transfer processes by appropriate approximations and corrections. The computations are performed in each gas-phase CFD cell in the computational domain. 


\subsection{Generalised 1D Model}

The generalised 1D model is constructed through analysing the heat transfer to and within an element in an idealised protected steel member assumed to be exposed to heat on two faces, as shown in Figure 1 below:

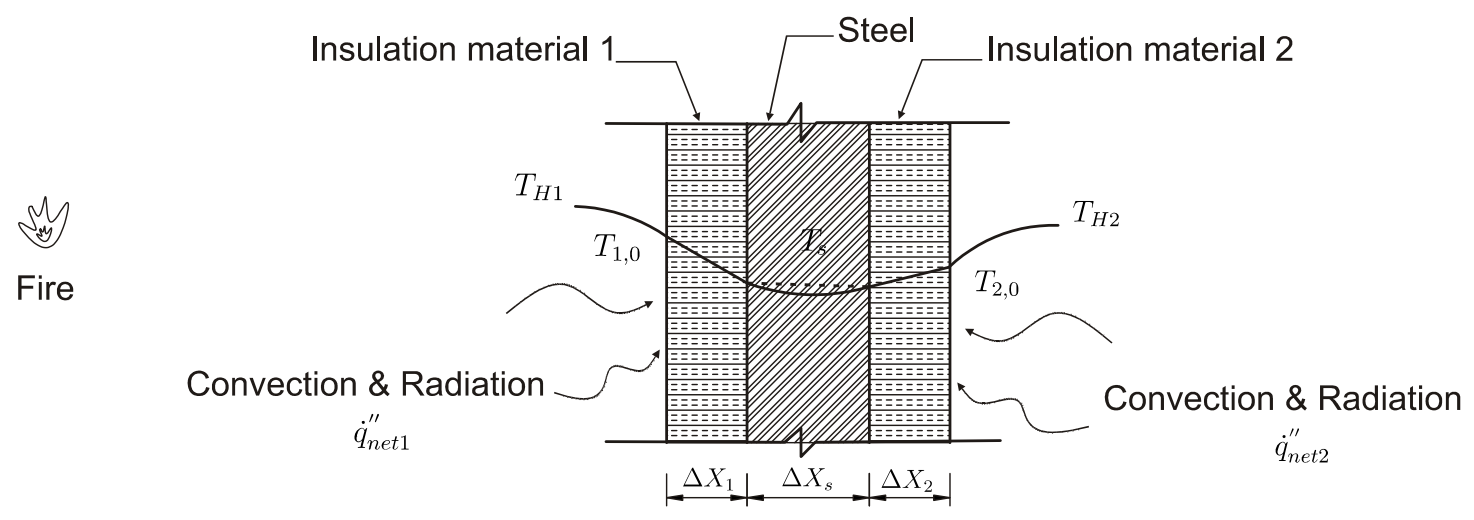

Figure 1. Schematic of Heat Transfer to Protected Steel Member

This element is supposed to be representative of a slice of a protected steel structure, e.g. a finite section of a flange or a web; two faces are used to allow for situations where the exposure conditions on each side might vary, encompassing also the case of hollow sections with very different exposures on the inside of the structure, though in that case the insulation thickness on the inside is reduced to zero.

The generalised 1D model provides a modelling framework which exploits a simple thermal penetration model for the protection coupled to an essentially lumped parameter representation of the steel heating. The governing equations for this model are derived by considering the net energy balance together with surface heat transfer boundary conditions [7] as given below:

Energy balance equation:

$$
\frac{\partial E_{\text {system }}}{\partial t}=\dot{q}_{\text {net }}^{\prime \prime}
$$

i.e.

$$
\begin{aligned}
& \rho_{S} \cdot c_{p s} \cdot \frac{\partial T_{S}}{\partial t} \cdot \Delta x_{S}+w_{p 1} \cdot \rho_{1} \cdot c_{p 1} \cdot \frac{\partial T_{1}}{\partial t} \cdot \Delta x_{1}+w_{p 2} \cdot \rho_{2} \cdot c_{p 2} \cdot \frac{\partial T_{2}}{\partial t} \cdot \Delta x_{2} \\
& =h_{c 1} \times\left(T_{H 1}^{(n)}-T_{1,0}^{(n)}\right)+\dot{q}_{r 1}^{\prime \prime}-\varepsilon_{m 1} \cdot \sigma \cdot T_{1,0}^{(n)_{4}}+h_{c 2} \times\left(T_{H 2}^{(n)}-T_{2,0}^{(n)}\right)+\dot{q}_{r 2}^{\prime \prime}-\varepsilon_{m 2} \cdot \sigma \cdot T_{2,0}^{(n)_{4}}
\end{aligned}
$$

The terms shown in the expanded equation here represent, respectively, the transient heating of the steel and protection layer on each side, and the convection, radiation and re-radiation for each surface of the protected member. A semi-empirical treatment is adopted for transient heating, allowing for spatially- and temporally-varying temperature gradients within the solid. The boundary conditions are supplied from the heat transfer solution for the surfaces, using the following equations:

$$
\dot{q}_{n e t 1}^{\prime \prime}=\frac{k_{1}}{w_{p 1} \Delta x_{1}} \cdot\left(T_{1,0}^{(n)}-T_{S}\right)
$$


i.e. $\quad h_{c 1} \times\left(T_{H 1}^{(n)}-T_{1,0}^{(n)}\right)+\dot{q}_{r 1}^{\prime \prime}-\varepsilon_{m 1} \cdot \sigma \cdot T_{1,0}^{(n)} 4=\frac{k_{1}}{w_{p 1} \Delta x_{1}} \cdot\left(T_{1,0}^{(n)}-T_{S}\right)$

$$
\dot{q}_{n e t 2}^{\prime \prime}=\frac{k_{2}}{w_{p 2} \Delta x_{2}} \cdot\left(T_{2,0}^{(n)}-T_{S}\right)
$$

i.e. $\quad h_{c 2} \times\left(T{ }_{H 2}^{(n)}-T_{2,0}^{(n)}\right)+\dot{q}_{r 2}^{\prime \prime}-\varepsilon_{m 2} \cdot \sigma \cdot T_{2,0}^{(n)} 4=\frac{k_{2}}{w_{p 2} \Delta x_{2}} \cdot\left(T_{2,0}^{(n)}-T_{S}\right)$

where:

$\sigma \quad$ is Stefan-Boltzmann constant $\left(5.67 \times 10^{-8} \mathrm{~W} / \mathrm{m}^{2} / \mathrm{K}^{4}\right)$;

$\dot{q}_{r 1}^{\prime \prime}, \dot{q}_{r 2}^{\prime \prime} \quad$ are incident heat fluxes on each side;

$T_{1,0}^{(n)}, T_{2,0}^{(n)} \quad$ are surface temperatures at gas/solid interfaces;

$T_{s}, T_{1}, T_{2} \quad$ are steel and average protection layer temperatures, respectively;

$h_{c 1}, h_{c 2} \quad$ are convection coefficients;

$\varepsilon_{m 1}, \varepsilon_{m 2} \quad$ are emissivities of protection layers;

$\rho_{s}, \rho_{1,} \rho_{2} \quad$ are densities of steel and protection layers, respectively;

$\Delta x_{s}, \Delta x_{1}, \quad$ are thicknesses of steel and protection layers, respectively;

$\Delta x_{2}$

$w_{p 1}, w_{p 2} \quad$ are weight factors of protection layers, defined in terms of the thermal

penetration depth of the protection, given in the form of Equation 4:

$w_{p}=\min \left\{\frac{A_{\text {actual }}}{A_{\text {model }}}=\frac{\delta}{\Delta x_{p}}, 1\right\}$

where

$$
\delta=2 \cdot\left(\frac{k_{p} \cdot t}{c_{p} \cdot \rho}\right)^{1 / 2}
$$

$c_{s}, c_{p 1}, c_{p 2} \quad$ are specific heat of steel and protection layers, respectively;

$k_{1}, k_{2} \quad$ are thermal conductivity parameters of protection layers.

The temperature/time dependent characteristics, including moisture and intumescence effects, are incorporated in certain parameters for generalisation of the methodology. The moisture effects were treated by varying the relevant thermal properties of the material, in particular, the specific heat and thermal conductivity, by means of an additional temperature-dependent source term in the energy equation representing latent heat effects. The treatment for moisture effects was described in detail in the previous paper [5] whilst intumescence is described in section 3 below.

It is well-known that the above situation is a strongly coupled problem, with the net heat fluxes at the gas-solid interface very much dependent on the surface temperature, but both also related to the transient thermal response of the structure itself. Numerical instabilities might become evident if inadequate solution procedures are used; these are overcome using a Newton-Raphson method to update the surface temperature from the heat transfer boundary condition governing equations and thereafter, with the updated surface temperature as a boundary condition, solving the overall energy balance equation (Eq. 1) with the Runge-Kutta method to obtain the steel temperature. 


\subsection{Quasi-3D Model}

Use of a fundamentally 1D treatment is essential, considering the costs of doing a full 3D analysis in every computational cell and including a sufficient number of parametric variations. However, adoption of a simple 1D model for thermal analysis could clearly lead to some modelling inaccuracies. These could in principle be in either direction, resulting in either conservative (over-design) or non-conservative (unsafe) results. The former aspect is not a major concern since the method is in any case far more flexible than other simple models, and by using generalised treatments conservatism is already greatly reduced. The latter aspect is a more obvious problem, and in order to overcome it, methods for treating important $2 \mathrm{D}$ and $3 \mathrm{D}$ effects are needed. A number of corrections factors have been implemented in the model, encompassing the factors indicated in Figure 2, i.e. the junction effect, end effect, heat sink effect and axial temperature gradient effect.

- Junction effect

This effect accounts for the effects of the connection between two different parts of a member under differential heating, i.e. where there is a temperature differential due to the fact that the exposure of one part of the structural component, e.g. a flange or a web, is dominant over that of a connected part.

- $\quad$ End effect

Along the length of the flange, the steel temperature changes, especially for the cells in the two ends of the flange, which might be a worst case position for temperature by virtue of the fact that they are exposed to heat arriving from two different directions, i.e. their heated surface is larger.

- Heat sink effect

It considers the thermal effects when a structural member is in contact with a ceiling slab.

- $\quad$ Axial temperature gradient

This effect accounts for the case when the structure passes from a cold layer into a hot layer, the temperature changes greatly with height, or where there are significant horizontal temperature gradients.

It is important to note that these effects are only critical where they negatively impact the performance of the member, i.e. increase the solid temperatures, and in the majority of cases the opposite is true, i.e. the default procedure is a good representation of the "worst" case. Thus, while it is vital to show that these possible corrections have been appropriately considered, their effect on the final results has been found to be fairly limited.

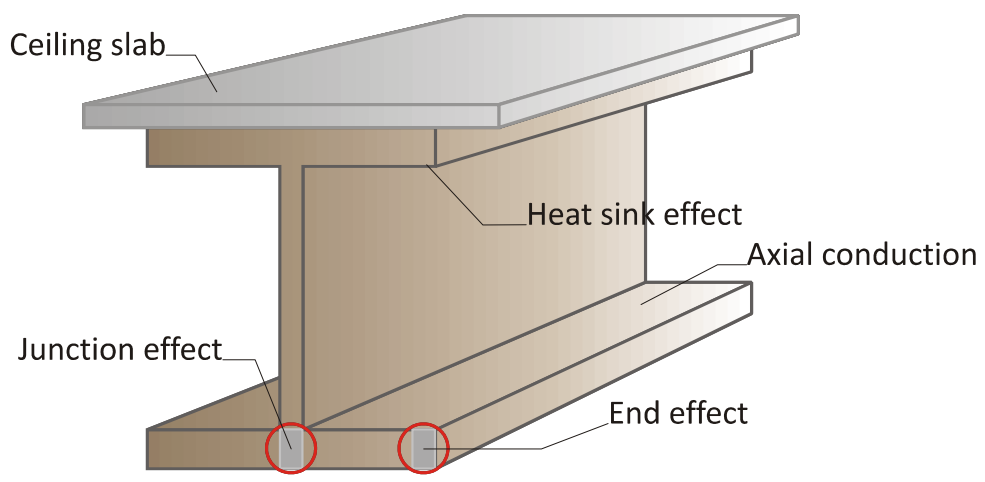

Figure 2. Cross-section of the Beam with Locations of Possible Correction Effects 


\section{MATERIAL PROPERTIES}

The aforementioned model might be considered as a reasonable representation of the fundamental aspects of the heat transfer phenomena. However, in practice, several factors are found to have a great impact on the transient response, in particular the thermal properties of the protection materials, which affect the surface temperature and thus the steel temperature. It is known that these properties are often strongly temperature/time dependant and the use of constant values may result in significant errors in some cases. The methodology developed here aims at generalising the thermal analysis to accommodate all important phenomena; conventional approaches to treatment of moisture effects have already been implemented, referencing modified specific heats and thermal conductivities [5]. This is now extended further to include the effects of intumescence, clearly of great practical relevance to the case of protected steelwork. In order to do so, geometrical and density variations must also be explicitly treated.

Intumescent materials are an increasingly popular form of fire protection, due to a number of advantages arising from the fact that they can be applied as thin, aesthetically pleasing, coatings either before or after construction $[8,9,10]$. When in contact with high temperatures, they will swell and form a layer of carbonaceous char which has much greater thickness than the initial state. The char subsequently acts as a thermal barrier to effectively protect the substrate against increase in temperature. Nevertheless, during the process of intumescence, the material properties are severely changed along with mass transport and endo- and exothermic reactions. These properties include thermal conductivity, specific heat, density and thickness of the intumescent layer.

Several research studies have been carried out to determine the effective intumescent thermal properties by experimental tests, in conjunction with some form of numerical analysis. These include bench-scale cone calorimeter tests and small-scale furnace tests on coated plates [11, 12], and furnace tests on cellular beams [13]. The first authors conducted studies on typical water-based and solvent-containing intumescent systems [10] and later on a high-performance material, i.e., epoxy resin containing boric acid and phosphate-based flame retardant [12]. The results from the former showed a significant slow down of temperature increase between $200-300^{\circ} \mathrm{C}$, due to intumescence, i.e. the formation of an insulating char and other co-acting energy absorbing processes; temperature influences during the latter tests also resolved a damping effect at $150^{\circ} \mathrm{C}$ due to the endothermic reaction of boric acid, which also releases water. Layer thickness effects were non-linear. Considering first the geometrical expansion, a simple conceptual model would suggest that thermal equivalence to a finite thickness problem can be achieved by simply scaling the thermal conductivity by the layer thickness, $d(=1 \mathrm{~m})$, giving an effective thermal conductivity, $k / d$. Density is scaled in the same way, and specific heat by the inverse of $d$, but these parameters always appear as factors of each other so these scalings vanish in the term $\rho c_{p}$.

The description of the temperature-dependent intumescent thickness, $d$, can be determined from an expression for the expansion ratio. We postulate that this will fit the general form:

\begin{tabular}{ll}
\hline$R=1$ & $T<T_{\text {lower }}$ \\
\hline$R=1+\frac{1}{2}\left(R_{f}-1\right)\left(\frac{T-T_{\text {lower }}}{T_{\text {mid }}-T_{\text {lower }}}\right)^{n}$ & $T_{\text {lower }} \leq T<T_{\text {mid }}$ \\
\hline$R=R_{f}-\frac{1}{2}\left(R_{f}-1\right)\left(\frac{T_{\text {upper }}-T}{T_{\text {upper }}-T_{\text {mid }}}\right)^{n}$ & $T_{\text {mid }} \leq T<T_{\text {upper }}$ \\
\hline$R=R_{f}$ & $T \geq T_{\text {upper }}$ \\
\hline
\end{tabular}




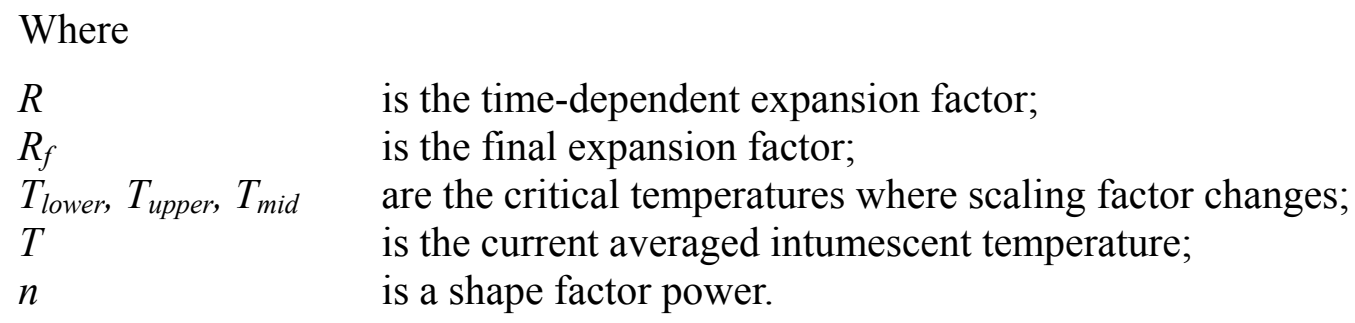

Here, besides the relevant temperatures limits, the critical controlling parameters are the shape factor $n$ and the overall expansion ratio $R_{f}$. An approximate calibration has been performed by comparison with test data, including the results of Bartholmai [12] giving a value of $n=2$. Taking an approximate temperature range from the DTG results of the latter study, and assuming $R_{f}=10$, gives the following curve:

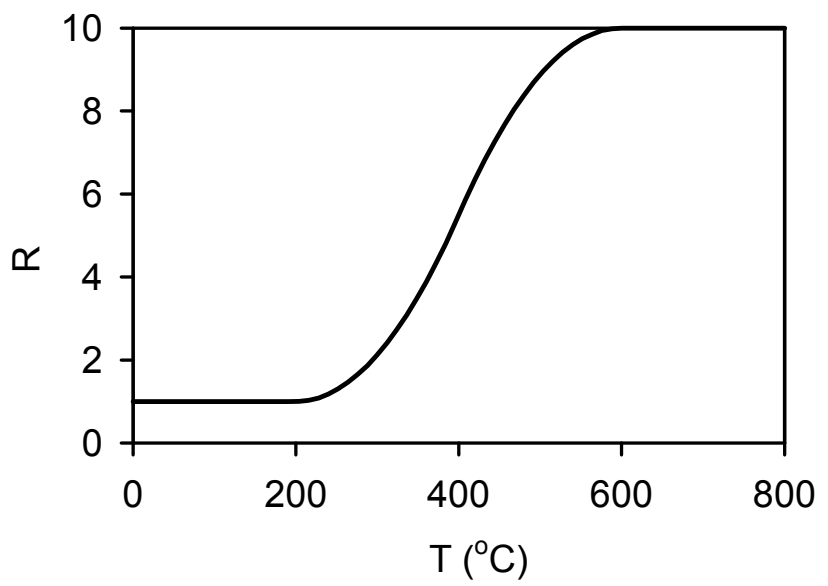

Figure 3. Scaling Factor R Change with Temperature

In fact, a variety of overall 1D expansion ratios have been reported in the literature, 10 [14] , 15-30[8], 50-220 [11].

The key parameter for the thermal model is the conductivity, or its scaled value, i.e. $k / d$. The conductivity itself is affected by fundamental changes in the material as it intumesces $[11,12,15]$. Unfortunately, the effect is non-linear and very dependent on initial thickness, and most pronounced at the smaller thicknesses typical of real applications; hence, there would appear to be no substitute for its direct experimental determination. A series of cone calorimeter tests hence have been carried out at the University of Edinburgh to investigate derivation of the temperature/time-dependent thermal properties of intumescent materials, as also reported previously [6]. Their intumescent expansion ratio was approximated by measuring peak char thickness after the test and their effective thermal conductivity deduced from calibration of a one-dimensional thermal model implemented in a spreadsheet. In the meanwhile, the various literature results would suggest an initial increase followed by a fall during intumescence and finally a sharp rise during material degradation. The final submodel for intumescent properties is constructed by combined consideration of the experimental findings with the literature results.

\section{MODEL VERIFICATION AND VALIDATION}

The above conceptual model has been implemented as a submodel called GeniSTELA within the SOFIE RANS CFD code [4]. Representative empirical values are adopted for some terms such as the initial conditions, the dry thermal properties, moisture content, etc., and their influence has been studied by exercising the model with different sets of input parameter values. The performance of 
the model was assessed by performing sensitivity studies, looking at the effects of a range of numerical and physical parameters. Comparisons were also made with the results from the EC3 protected member equation [1].

The case used for verification studies is the protected steel indicative, UC254x254/73, in the full-scale tests on a $12 \times 12 \mathrm{~m}$ compartment undertaken at BRE Cardington [16]; this member was protected with about $25 \mathrm{~mm}$ of Fendolite MII sprayed fibre (baseline $\rho=680 \mathrm{~kg} / \mathrm{m}^{3}, k=0.19 \mathrm{~W} / \mathrm{m} / \mathrm{K}$ ). Figure 5 shows the test compartment while Figure 6 shows the SOFIE temperature predictions.

In the test a variety of thermal parameter measurements were made, encompassing conditions in the gas phase (temperatures, velocities and heat fluxes) and in the solid phase (steel temperatures in protected beams, columns and indicatives, with and without protection); this study also serves for the validation of the model, comparing the model predictions with the measured steel temperatures in the protected indicative.

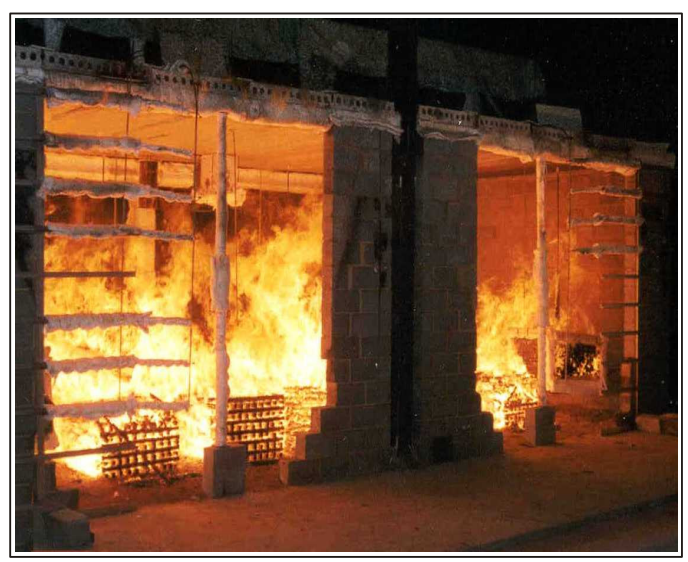

Figure 5. BRE Large Compartment Fire Test Figure 6. BRE Large Compartment Fire Modelling

\section{RESULTS}

\subsection{Simulation Results}

Gas and steel temperatures were computed using SOFIE and the coupled GeniSTELA code. In qualitative terms the results showed the expected differences in steel and gas temperature fields, with relatively higher steel temperatures within the depth of the compartment compared to the openings. This is consistent with the fact that the thermal exposures are more severe deeper into the fire [16], and the model predictions from GeniSTELA are heavily influenced by the radiative terms, $\dot{q}_{r}^{\prime \prime}$, derived directly from the CFD calculation.

Figure 7 shows the temperature predictions for the protected indicative within the compartment. There is a large temperature gradient across the protection. 


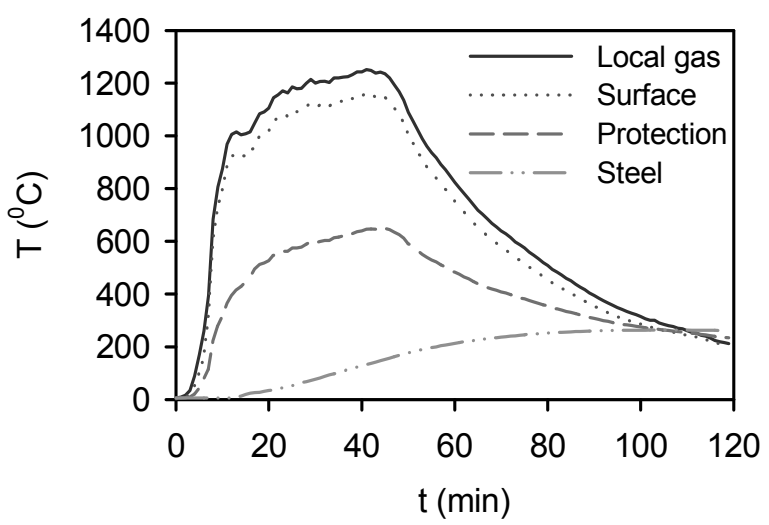

Figure 7. Temperatures at Protected Indicative, Test 8

Figure 8 shows a comparison of the predictions of steel temperature with the test together with EC3 prediction. The latter exceeds the measure temperature reflecting some conservatism in this semi-empirical method, while the prediction from GeniSTELA indicates a sufficient match with the test.

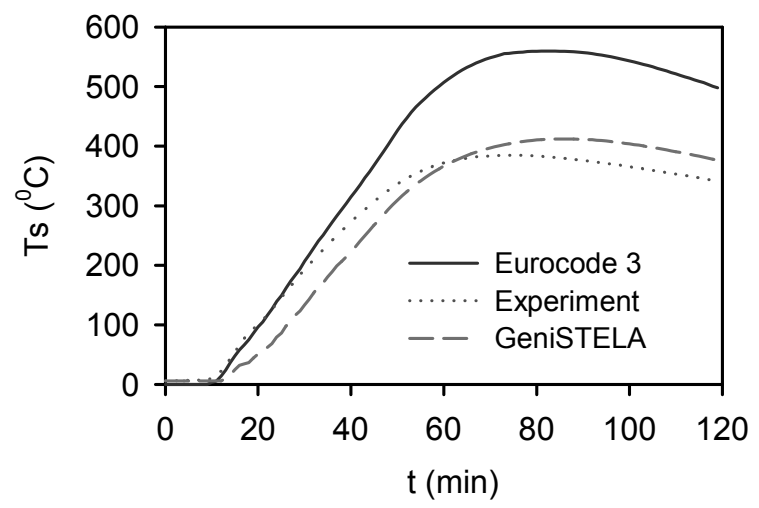

Figure 8. Comparison of Steel Temperatures

\subsection{Sensitivity Study Results}

Some results from the sensitivity study are shown in Figures $9 \sim 11$ for the effects of changing the steel flange thickness (spanning UC 254x254/73,107,167), the protection thickness (12.5 to 50mm) and the protection thermal conductivity $(0.1$ to 0.4$)$. The results for changing the protection thermal conductivity mirror the latter, and show the expected strong influence of protection properties.

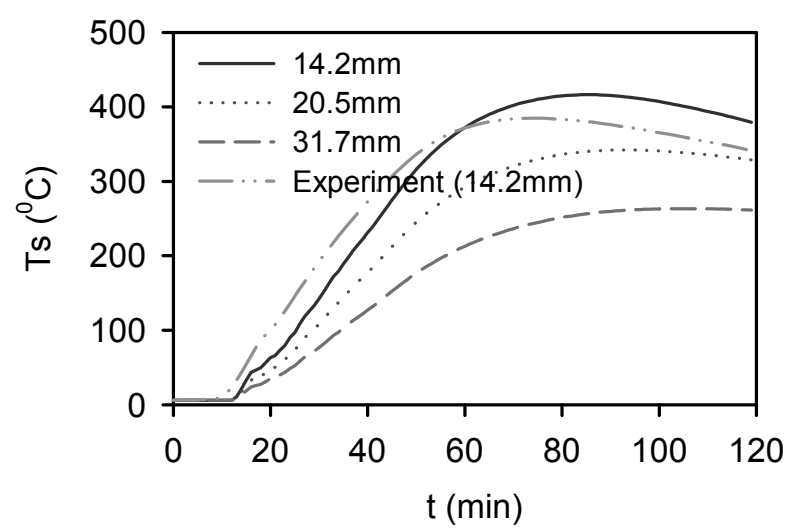

Figure 9. Effect of Flange Thickness on Steel Temperature 


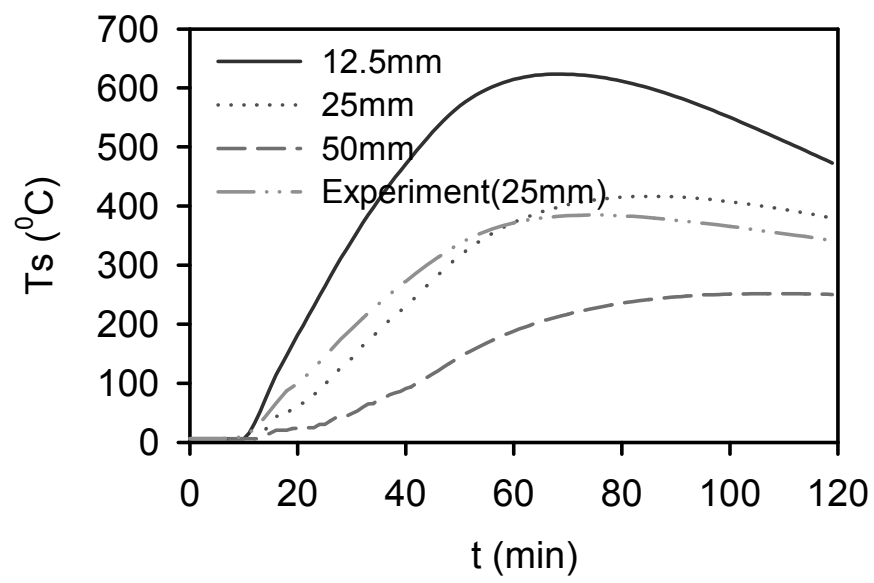

Figure 10. Effect of Protection Thicknesses on Steel Temperature

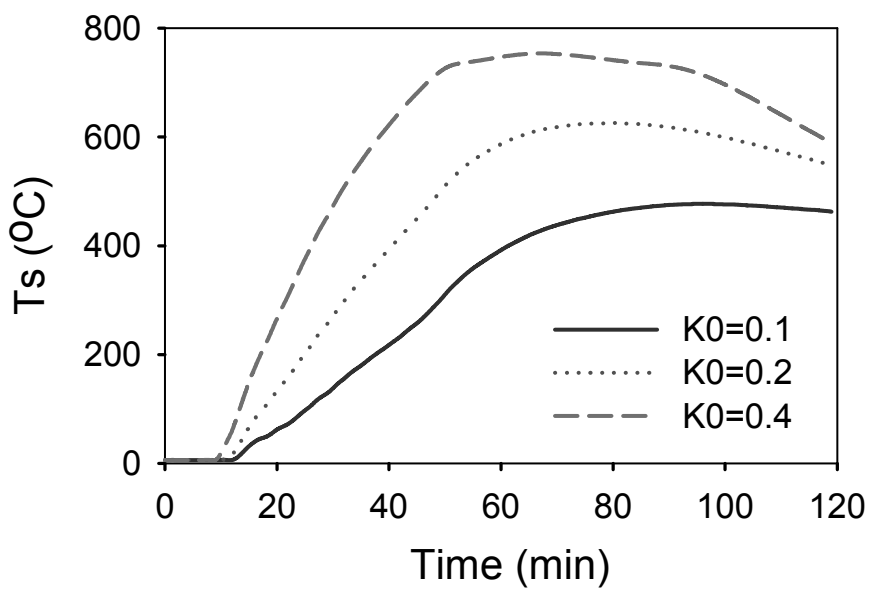

Figure 11. Effect of Protection Thermal Conductivity on Steel Temperature

Some other effects such as the moisture effect, the emissivity effect and the intumescent protection effect have also been examined during the case study. It is found that the moisture content could be an important factor within certain temperature range before the moisture has vanished. The emissivity effect has been studied by comparing steel temperature predictions from a range of emissivity values representing high emissivity (above 0.5 ) and low emissivity (below or equal to 0.5 ) either for the fire or for the member. It is found that the fire emissivity doesn't affect the final steel temperature prediction but that the member surface emissivity is a key factor that may influence the temperature rise in the steel. But overall, the emissivity has a low-order effect on performance especially for the start of the heating regime when convective heat transfer is dominant. This point is consistent with the findings by Staggs and Phylaktou [17]. The intumescent protection has strong effects on the final results regarding to the type of intumescent protection and protection thickness. Those interesting findings during the sensitivity study facilitate the further implementation of GeniSTELA results into a newly developed code called GeniSTRUC (Generalised STRUCtural Analysis) [18] for a generalised analysis of structural mechanical responses. Here, the GeniSTELA calculated steel temperature at each cell location of the structural member would be implemented via GeniSTRUC to the related location for structural analysis. The initial GeniSTRUC results indicate that normally the failure happens as expected in the middle cell of the beam but not in the end cell. This point demonstrates the practical usage of GeniSTELA in the structural fire design field. 


\section{COMPUTATIONAL REQUIREMENTS STUDY}

In order to examine the practical use of this methodology, the overall computational requirements have been assessed in terms of the CPU time usage. Also, the potential for reducing the frequency of the calls to the GeniSTELA steel temperature solver has been explored by changing this from the default of once every 10 iterations. This default was determined to match the usual frequency of calls to the radiation solver, since one of the key drivers of the thermal response is the radiation field and in most cases there is no benefit in recomputing steel temperatures if this has not been updated. The change in GeniSTELA call frequency is realised by introducing a timestep factor variable (tfactor) in the model in order to increase the intervals between calls.

The results showed that GeniSTELA uses around $1 \%$ of the CPU time for the flow solver, including radiation, when called at the default interval of 10 iterations. Simulations were then undertaken with a tfactor value of 10 for a localised fire scenario, with a constant fire size, having realistic steel temperature increases in 10 minutes. Figure 12 shows the results for the respective steel temperature predictions, confirming that even when called only once per every 100 main solver iterations, i.e. with just six calls of the GeniSTELA solver altogether, there is a very small effect on the final steel temperature result, especially at latter times. Figure 13 shows the evolution of the percentage temperature difference. It is obvious that the discrepancy is only important in the early stage, with the maximum difference being only of order $8 \%$, mostly within $3 \%$. Using an intermediate value of tfactor $=2$ gave results much closer to the default case, with a maximum discrepancy of only $1.5 \%$.

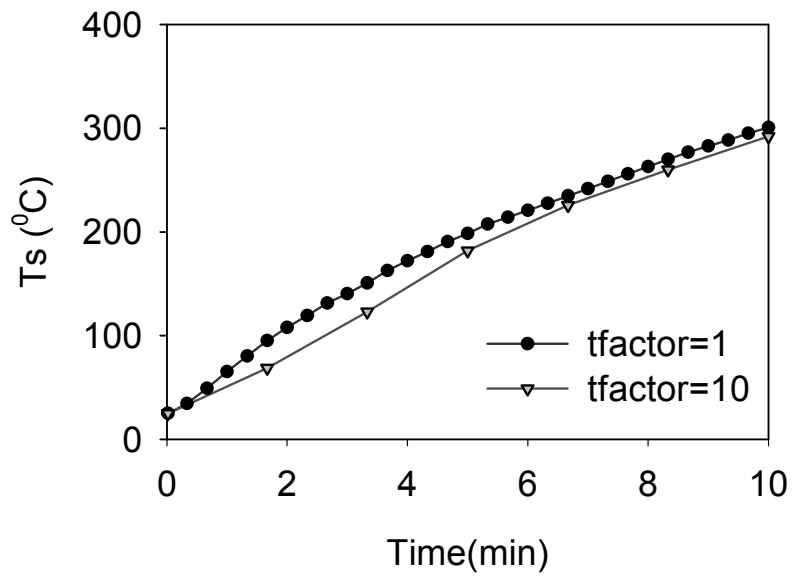

Figure 12. Predicted Steel Temperatures with tfactor $=1$ and 10

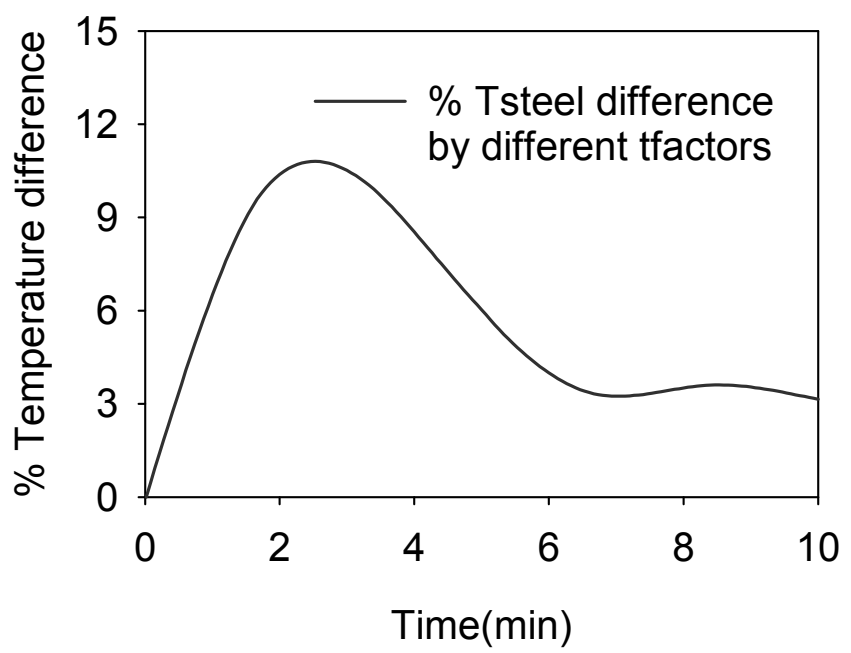

Figure 13. Difference in Steel Temperature Against Time Using tfactor $=1$ and 10 
The above findings are of course only of relevance for this particular steady fire, and for more general cases, where the heat release rate may be changing rapidly, higher frequencies may be required. In practice, the frequency of the GeniSTELA call could be adjusted by automatic selection linked to heating rates, in order to achieve the best efficiency. Nevertheless, this initial study suggests that a full set of parametric calculations (10-100 cases) could be afforded without any significant compromise of accuracy, before the GeniSTELA analysis becomes the dominant part of the computation.

\section{CONCLUSIONS}

A generalised methodology for thermal analysis of protected steel structures in fire is described. A framework for the inclusion of treatments for intumescence effects has been established, with provision for simple calibration in the case of each specific formulation of interest. The GeniSTELA implementation of the method is based on simultaneous computations spanning the range of cases of interest, providing a novel generalised methodology. The results confirm the sufficiency of the algorithms adopted and comparisons with measurements in a post-flashover compartment fire test are satisfactory. Computational demands are found to be acceptable. Strong dependencies on the thermal properties of the protection materials are observed in the sensitivity studies. These results serve to illustrate the importance of using generalised methodologies in tackling thermal response problems and suggest a new approach to structural fire safety engineering design.

\section{ACKNOWLEDGEMENTS}

The first author acknowledges the support of her industrial sponsor, the BRE Trust, and the School of Engineering. Thanks also to the members of BRE Centre for Fire Safety Engineering, and technical assistants, for their support during the research. Useful comments on intumescent properties were received from Yong Wang and Matthias Bartholmai, and Daniel Joyeux and Mario Fontana via IAFSS email list.

The work reported in this paper has formed part of FireGrid, www.firegrid.org. This project was/is co-funded by the Technology Strategy Board's Collaborative Research and Development programme, following an open competition.

\section{NOMENCLATURE}

$c_{p s}, c_{p 1}, c_{p 2} \quad$ specific heat capacity of steel and protection layers 1,2 , respectively $(\mathrm{J} / \mathrm{kg} / \mathrm{K})$

$d \quad$ intumescent layer thickness (m)

$E_{\text {system }} \quad$ total energy in the system $(\mathrm{J})$

$h_{c 1}, h_{c 2} \quad$ convection coefficient on sides 1,2 , respectively $\left(\mathrm{W} / \mathrm{m}^{2} / \mathrm{K}\right)$

$k_{1}, k_{2} \quad$ thermal conductivity of protection layers 1,2 , respectively $(\mathrm{W} / \mathrm{m} / \mathrm{K})$

$\dot{q}_{n e t 1}^{\prime \prime}, \dot{q}_{n e t 2}^{\prime \prime} \quad$ net heat flux on sides 1,2 , respectively $\left(\mathrm{W} / \mathrm{m}^{2}\right)$

$\dot{q}_{r 1}^{\prime \prime}, \dot{q}_{r 2}^{\prime \prime} \quad$ incident heat flux on sides 1,2 , respectively $\left(\mathrm{W} / \mathrm{m}^{2}\right)$

$t \quad$ time (s)

$T_{H 1}, T_{H 2} \quad$ gas temperature on sides 1,2 , respectively $(\mathrm{K})$

$T_{1,0}^{(n)}, T_{2,0}^{(n)} \quad$ surface temperature at gas/solid interface on sides 1,2 at $n^{\text {th }}$ time-step, respectively $(\mathrm{K})$ 


$\begin{array}{ll}T_{s}, T_{1}, T_{2} & \text { average temperatures of steel and protection layers on sides } 1,2 \text {, respective } \\ & \text { (K) } \\ w_{p 1}, w_{p 2} & \text { "weight factors" of protection layers on sides } 1,2 \text {, respectively }(-) \\ \Delta x_{s}, \Delta x_{1}, \Delta x_{2} & \text { thickness of steel and protection layers on sides } 1,2 \text {, respectively }(\mathrm{m}) \\ \delta_{1}, \delta_{2} & \text { thermal penetration depth on sides } 1,2 \text {, respectively (m) } \\ \varepsilon_{m 1}, \varepsilon_{m 2} & \text { emissivity of protection layers on sides } 1,2 \text {, respectively (-) } \\ \rho_{s}, \rho_{1}, \rho_{2} & \text { density of steel and protection layers on sides } 1,2 \text {, respectively }\left(\mathrm{kg} / \mathrm{m}^{3}\right) \\ \sigma & \text { Stefan-Boltzmann constant }\left(5.67 \times 10^{-8} \mathrm{~W} / \mathrm{m}^{2} / \mathrm{K}^{4}\right)\end{array}$

\section{REFERENCES}

[1] British Standards Institution, "Eurocode 3: Design of Steel Structures - Part 1-2: General Rules - Structure Fire Design", 2002.

[2] Welch, S., Miles, S. Kumar, S., Lemaire, T. and Chan, A., "FIRESTRUC - Integrating Advanced Three-dimensional Modelling Methodologies for Predicting Thermo-mechanical Behaviour of Steel and Composite Structures Subjected to Natural Fires", Proceedings of $9^{\text {th }}$ International IAFSS Symposium, 2008, Karlsruhe, Germany.

[3] Kumar, S., Welch, S., Miles, S.D., Cajot, L.-G., Haller, M., Ojanguren, M., Barco, J., Hostikka, S., Max, U. and Röhrle, A., "Natural Fire Safety Concept - The Development and Validation of a CFD-based Engineering Methodology for Evaluating Thermal Action on Steel and Composite Structures", European Commission Report EUR 21444 EN, 2005, Vol. 150 pp. ISBN 92-894-9594-4.

[4] Lewis, M.J., Moss, J.B. and Rubini, P.A., "CFD Modelling of Combustion and Heat Transfer in Compartment Fires", Proceedings of $5^{\text {th }}$ International Symposium on Fire Safety Science, 1997, pp. 463-474.

[5] Liang, H. and Welch, S., "A Novel Engineering Tool for Thermal Analysis of Structural Members in Natural Fires", Proceedings of $4^{\text {th }}$ International Workshop on Structures in Fire, 2006, Aveiro, Portugal.

Also available: $h$ ttp://hdl.handle.net/1842/1985

[6] Liang, H., Welch, S. Stratford, T. and Kinsella, E.V., "Development and Validation of a Generalised Engineering Methodology for Thermal Analysis of Structural Members in Fire", Proceedings of $5^{\text {th }}$ International Seminar Fire \& Explosion Hazards, 2007, Edinburgh, UK.

Also available: http://hdl.handle.net/1842/1990

[7] Carslaw, H.S. and Jaeger, J.C., "Conduction of Heat in Solids", Oxford University, 1959.

[8] Goode, M.G. (ed.), "Fire Protection of Structural Steel in High-rise Buildings", NIST GCR 04-872, 2004.

[9] Jimenez, M., Duquesne, S. and Bourbigot, S., "Characterisation of the Performance of An Intumescent Fire Protective Coating", Surface and Coatings Technology, 2006, Vol. 201, issue 3-4, pp. 979-987.

[10] Bailey, C.G., "Advances in Fire Engineering Design of Steel Structures", Proceedings of ICE, Structures and Buildings, 2006, Vol. 159, issue SBI, pp. 21-35.

[11] Bartholmai, M., Schriever, R. and Schartel, B., "Influence of External Heat Flux and Coating Thickness on the Thermal Properties of Two Different Intumescent Coatings using Cone Calorimeter and Numerical Analysis", Fire and Materials, 2003, Vol. ?????, pp. 151-162.

[12] Bartholmai, M. and Schartel, B., "Assessing the Performance of Intumescent Coatings using Bench-scaled Cone Calorimeter and Finite Difference Simulations", Fire and Materials, 2007, Vol. 31, pp. 187-205. 
[13] Bailey, C.G., "Indicative Fire Tests to Investigate the Behaviour of Cellular Beams Protected with Intumescent Coatings", Fire Safety Journal, 2006, Vol. 36, No. 8, pp. 689-700.

[14] Desanghere, S. and Joyeux, D., "Development of Design Rules for the Fire Behaviour of External Steel Structures", ECSC Project No. 7210-PR-380, Final Report, 2005.

[15] Tan, K.H., Wang, Z.H. and Au, S.K., "Heat Transfer Analysis for Steelwork Insulated by Intumescent Paint Exposed to Standard Fire Conditions", Proceedings of $3^{\text {rd }}$ International Workshop on Structures in Fire, 2004, Ottawa, Canada.

[16] Welch, S., Jowsey, A., Deeny, S., Morgan, R. and Torero, J.L., "BRE Large Compartment Fire Tests - Characterising Post-flashover Fires for Model Validation", Fire Safety Journal, 2007, Vol. 42, pp. 548-567.

[17] Staggs, J. and Phylaktou, H., "The Effects of Emissivity on the Performance of Steel in Furnace Tests", Fire Safety Journal, 2008, Vol. 43, pp. 1-10.

[18] Liang, H., Welch, S., Faure, L., and Gillie, M., "Proceedings of International Conference on Applications of Structural Fire Engineering", 2009, Prague, Czech Republic. 


\title{
A SPECIFIC PROCEDURE FOR SEISMIC DESIGN OF COLD-FORMED STEEL HOUSING
}

\author{
R. Landolfo ${ }^{1, *}$, L. Fiorino $^{2}$ and O. Iuorio ${ }^{3}$ \\ ${ }^{I}$ Full Professor, Department of Constructions and Mathematical Methods in Architecture, \\ Faculty of Architecture, University of Naples Federico II, Naples, Italy \\ ${ }^{2}$ Research Fellow, Department of Structural Engineering, University of Naples "Federico II", Naples, Italy \\ ${ }^{3}$ PhD, Department of Design, Rehabilitation and Control of Architectural Structures, \\ University of Chieti/Pescara "G. D'Annunzio", Pescara, Italy \\ *(Corresponding author: E-mail: landolfo@unina.it)
}

Received: 4 June 2008; Revised: 19 September 2008; Accepted: 21 October 2008

\begin{abstract}
In the last years, the seismic performance of cold formed steel (CFS) systems has been object of study by a large number of research teams. The main parameters influencing the system behaviour under horizontal loads have been defined and the possible mechanisms of collapse have been identified. Therefore, at the moment, the development of upgraded specifications and design tools that could be readily used by designer is a must. For these reasons, this paper aims to propose a seismic design procedure that allows the shear wall components to be defined in few steps. The procedure is based on the recognized assumption that the best performance of a CFS shear wall is achieved when the sheathing-to-stud fasteners failure is reached. Moreover, it accounts the results of previous studies that defined the relation between behaviour of shear wall and fastener spacing. Hence, by defining three nomographs, the proposed procedure allows the sheathing-to-stud fasteners and all the other shear wall components to be defined. The latter are determined in accordance with capacity design criteria. Finally, the applicability of the presented procedure is verified through a case study.
\end{abstract}

Keywords: Design nomographs, cold-formed steel, housing, linear dynamic analysis, nonlinear static analysis, seismic design, sheathing panels

\section{INTRODUCTION}

The growing demand of high performance housing is increasing the interest for cold-formed steel (CFS) structures. These structures are realized with a repetitive number of light gauge cold-rolled steel profiles, produced off-site in controlled environment and, then, assembled on-site with mechanical connections. These characteristics provide them lightness, rapid on-site erection, dimensional stability, high structural performance and considerable potential for recycling and reuse of all the materials. In particular, the spreading use of these constructions in countries as Japan shows the wide applicability of these systems also in seismic zones (Figure1). The global horizontal response of CFS structures under seismic actions basically depends on the shear response of CFS walls that are usually sheathed with metal sheets or panels (sandwich panels, wood-based or gypsum-based panels). Moreover, when the sheathing has adequate strength and stiffness and it is effectively connected with the frame, then the interaction between profiles, sheathing panels and sheathing-to-frame connections can be advantageously taken into account in the structural analysis. In this case, the definition of shear wall elements (studs, sheathing panels, sheathing-to-frame connections, and frame-to-foundation anchors), that constitute the main lateral resisting system in case of seismic actions, is a key point for the design. Currently, a relatively large database of experimental investigations carried out on different CFS wall assemblies subjected to in plane shear loading has been obtained by Gad et al. [1], Tian et al. [2], Branston et al. [3], Fulop and Dubina [4], Landolfo et al. [5], Langea and Naujoks [6], Serrette et al. [7]. As result, the main parameters influencing the shear behaviour of walls have been identified. As a consequence, some general guidelines for the design of sheathed CFS walls have been recognized, as the use of oversized chord studs and shear and hold-down anchors. Therefore, if CFS walls are designed 
following these basic guidelines, then a good lateral response, characterized by sheathing-to-frame connections collapse can be obtained. Nowadays, moving from these cardinal points, different design methodologies are available (UBC [8] and IBC [9] design tables), but the development of tools that could readily be used by designers should be improved. In an attempt to give a contribution on this topic, a specific seismic design procedure is presented hereafter. Finally, the applicability of the proposed designed procedure is investigated through a case study.
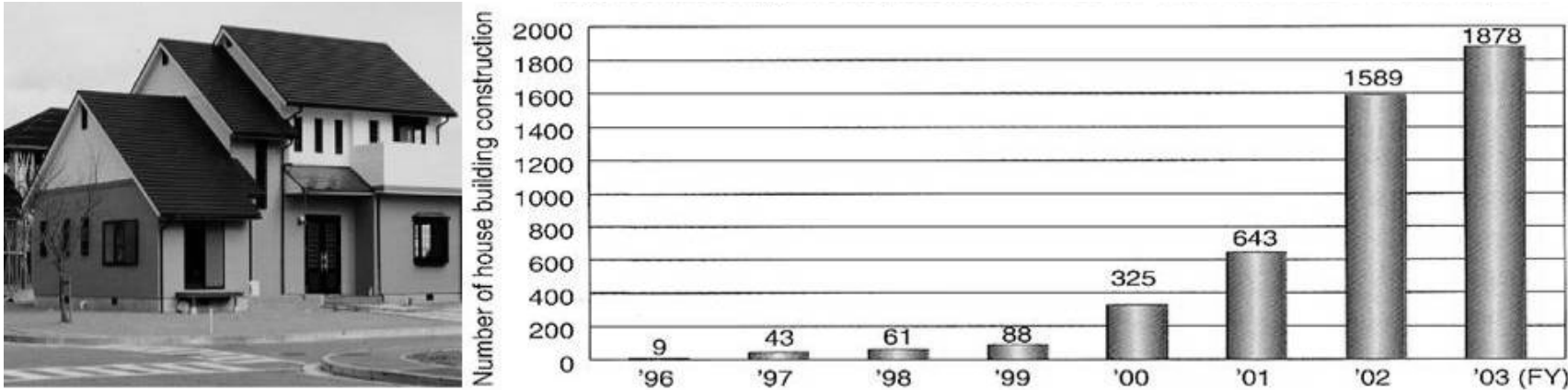

Figure 1. CFS Housing in Japan: Growing Construction in the Last Ten Years

\section{THE PROPOSED DESIGN PROCEDURE}

The proposed seismic design procedure refers to CFS walls sheathed with wood-based or gypsum-based panels, in which the wall components (Figure 2) are designed in such a way to promote the sheathing-to-frame fastener failure. The design procedure can be summarized in three consecutive phases (Figure 3):

1) definition of the wall geometry (wall height $h$, stud spacing $c$ ) and some parameters of the wall components: sheathing (type, thickness, orientation), frame (steel grade, stud size, with the exception of the thickness $t$, track size) sheathing-to-frame connections (type, interior spacing $p$, edge distance $e$ ), hold-down anchors (type) and shear anchors (type).

2) evaluation of the sheathing-to-frame fastener exterior spacing $s$;

3) evaluation of other design parameters (stud thickness $t$, hold-down anchor diameter $\phi$, shear anchor spacing $a$ ).

In this design procedure, the selection of the "assigned" design parameters (phase 1), usually derives from architectural and technological considerations and design for vertical loads. Therefore, no additional information are provided hereafter. Moreover, only the assessment of the sheathing-to-frame fasteners exterior spacing $(s)$ directly derives from seismic analysis results (phase 2), while the definition of stud thickness $(t)$, hold-down anchor diameter $(\phi)$, and shear anchor spacing $(a)$ is carried out on the basis of "capacity design" criteria (phase 3).

More details about the hypothesis and limit of applicability of this proposed procedure can be found in [10] 


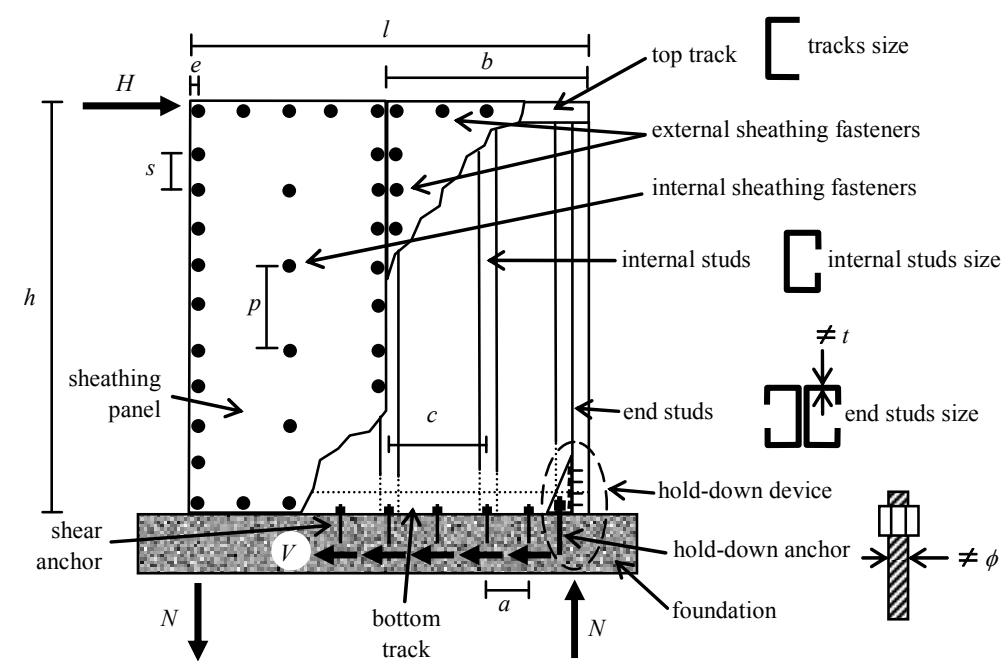

$N$ : axial force acting on the end stud / hold-down anchor, $V$ : shear force acting on a shear anchor

Figure 2. Geometry and Component of Typical Sheathed CFS Shear Wall

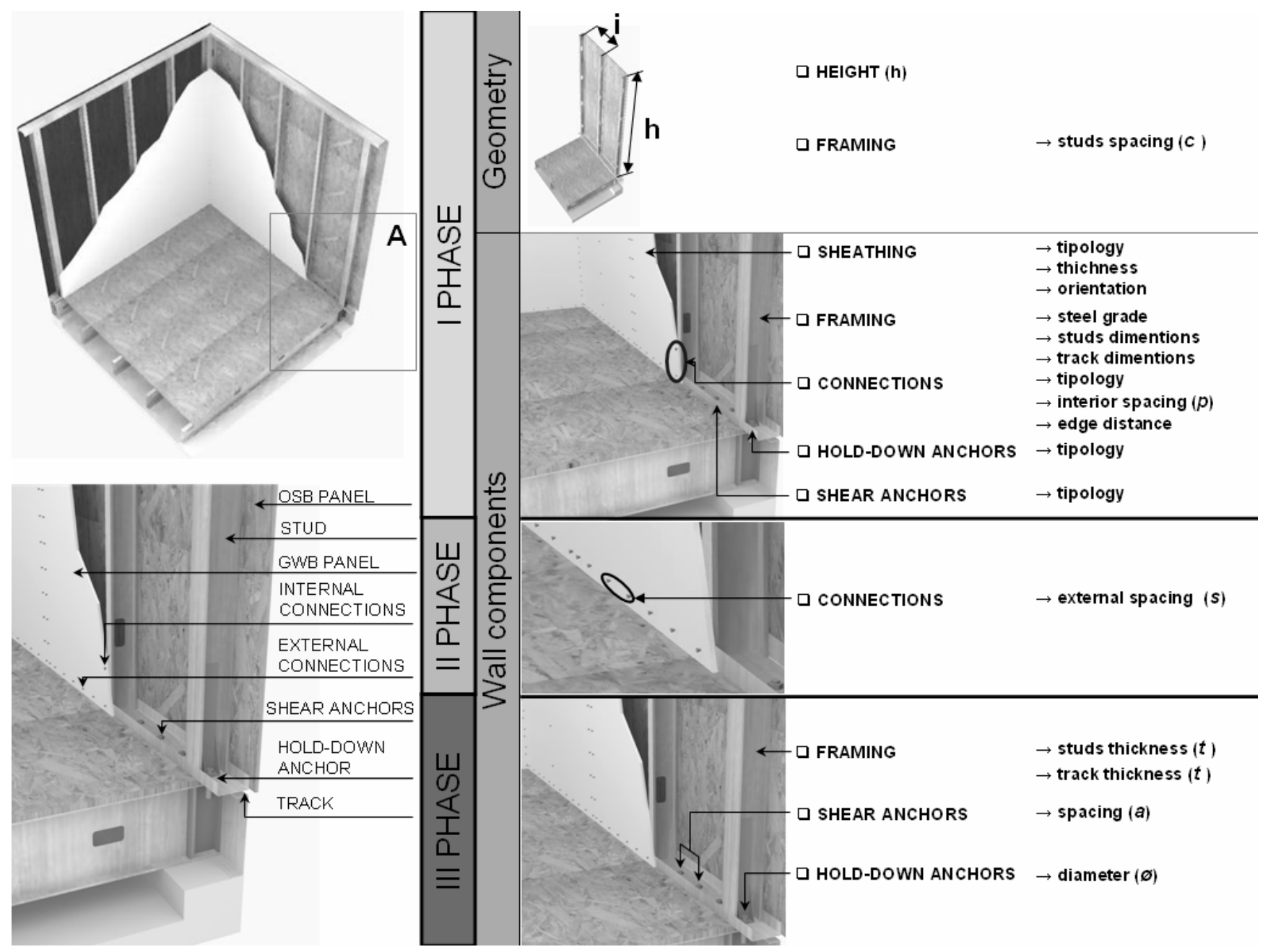

Figure 3. Proposed Design Procedure Schema 


\section{PHASE 2: EXTERIOR SPACING EVALUATION}

\subsection{Linear Dynamic Seismic Procedure}

When the linear dynamic (LD) procedure is selected for the seismic analysis, a force-based design approach is usually used, in which the inelastic behaviour and the structural overstrength are taken into account by the seismic force modification factors. In this case, the comparison between seismic capacity and demand, in terms of forces, shall satisfy the following equation:

$$
H_{C} \geq H_{D}
$$

where $H_{C}$ and $H_{D}$ are the seismic strength capacity and seismic action demand, respectively.

In particular, the seismic action $\left(H_{D}\right)$, which represents the horizontal in-plane force acting on the wall, can be evaluated through the following well known relationships:

$$
H_{D}=S_{a} \cdot \frac{w}{g} S_{a}=f_{S_{a} T}(T, \xi) T=2 \pi \sqrt{\frac{w / g}{K}}
$$

where: $S_{a}$ is the spectral acceleration; $f_{S a T}$ is the spectral acceleration function; $T$ is the structural period; $\xi$ is the viscous damping ratio; $w$ is the seismic weight; $g$ is the gravity acceleration; and $K$ is the wall shear stiffness, which can be expressed as a function of the sheathing fasteners exterior spacing $(s)$ :

$$
K=f_{K}(s)
$$

Also the seismic resistance $\left(H_{C}\right)$, which represents the wall shear strength, can be obtained as a function of $s$ :

$$
H_{C}=f_{H}(s)
$$

Therefore, for each value of exterior spacing $(s)$, if the functions $f_{S a T}, f_{K}$, and $f_{R}$ are known, then for fixed values of seismic weight $(w)$ and viscous damping ratio $(\xi)$, both seismic action $\left(H_{D}\right)$ and resistance $\left(H_{C}\right)$ can be calculated and compared by using Eq. (2) through (4) until Eq. (1) is satisfied. This design procedure can be represented graphically by means of the "LD" nomograph, as shown in Figure 4.

\subsection{Nonlinear Static Seismic Procedure}

When the seismic analysis is performed by means of the nonlinear static (NS) procedure, the inelastic behaviour and the structural overstrength are directly considered and the comparison between seismic capacity and demand can be achieved in terms of displacements (displacement-based approach):

$d_{C} \geq d_{D}$

where $d_{C}$ and $d_{D}$ are the seismic displacement capacity and seismic displacement demand, respectively. 


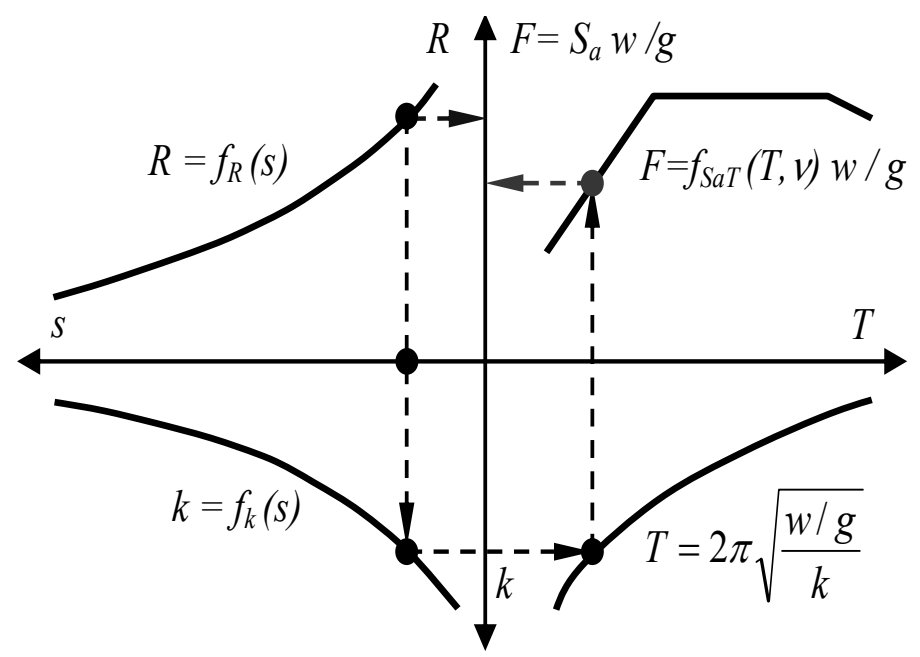

Figure 4. "LD” Nomographs

This comparison can readily be performed by means of the well known acceleration-displacement spectrum, in which the demand and capacity spectra are represented together. In particular, the demand spectrum can be obtained as follows:

$$
S_{a}=f_{\text {Sad }}(d, \xi)
$$

where $f_{\text {Sad }}$ is the spectral acceleration function, and $d$ is the generic displacement.

The capacity spectrum, instead, can be represented by an elastic-plastic curve, which is drawn by defining the yield $(Y)$ and ultimate $(U)$ limit points:

$$
Y\left(d_{y} ; \frac{H_{C}}{w} g\right), \quad U\left(d_{u} ; \frac{H_{C}}{w} g\right)
$$

where the yield displacement $\left(d_{y}\right)$ and ultimate displacement $\left(d_{u}\right)$ can be obtained as function of the exterior spacing $(s)$

$$
d_{y}=f_{y}(s)
$$

Therefore, for each value of exterior spacing $(s)$, if the functions $f_{S a d}, f_{H}, f_{y}$, and $f_{u}$ are known, then for fixed values of seismic weight $(w)$ and viscous damping ratio $(\xi)$, both seismic demand $\left(d_{D}\right)$ and capacity $\left(d_{C}\right)$ can be calculated and compared by using Eq. (6) through (8) until Eq. (5) be satisfied. This design procedure can be represented graphically by means of the "NS" nomograph shown in Figure 5. 


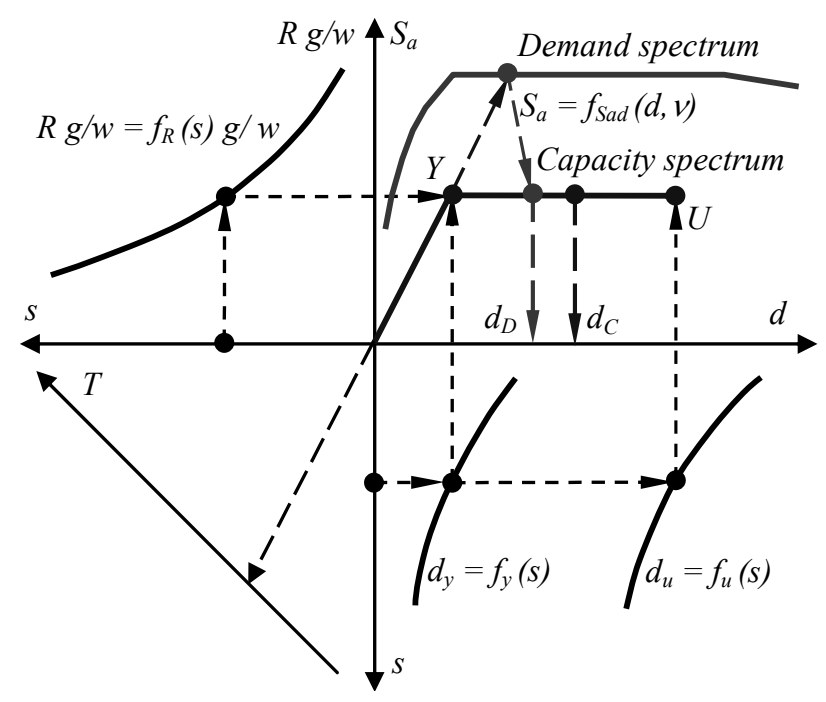

Figure 5. "NS" Nomograph

\section{PHASE 3: EVALUATION OF THE OTHER DESIGN PARAMETERS}

When the sheathing-to-frame fastener exterior spacing $(s)$ has been defined, then the evaluation of stud thickness $(t)$, hold-down anchor diameter $(\phi)$, and shear anchor spacing $(a)$ need to complete the wall design. The design procedure to define these last parameters can be represented graphically by means of an "OC" nomograph (Figure 6), in which the wall shear strength per wall unit length $\left(\bar{H}_{C}\right)$ corresponding to the resistance of sheathing fasteners $\left(\bar{H}_{C, f}\right)$, studs $\left(\bar{H}_{C, s}\right)$, hold-down anchors $\left(\bar{H}_{C, h a}\right)$ and shear anchors $\left(\bar{H}_{C, s a}\right)$ are represented together as function of the exterior spacing $(s)$. In particular, $\bar{H}_{C, f}$ can be obtained by knowing the function given in Eq. (4), while $\bar{H}_{C, s}, \bar{H}_{C, h a}$, and $\bar{H}_{C, s a}$ are calculated starting from the stud compression axial strength $\left(N_{s}\right)$, tension axial strength of hold-down anchor $\left(N_{h a}\right)$, and shear strength of the shear anchor $\left(V_{s a}\right)$ :

$$
\bar{H}_{C, s}=\frac{H_{C, s}}{l}=\frac{N_{s}}{h}, \quad \bar{H}_{C, h a}=\frac{H_{C, h a}}{l}=\frac{N_{h a}}{h}, \quad \bar{H}_{C, s a}=V_{s a}\left(\frac{1}{a}+\frac{1}{l}\right)
$$

where $l$ and $h$ are the wall length and height, respectively.

The unit wall strength corresponding to the resistance of sheathing fasteners $\left(\bar{H}_{C, f}\right)$ decreases when the exterior spacing $(s)$ increases, while $\bar{H}_{C, s}, \bar{H}_{C, h a}$, and $\bar{H}_{C, s a}$ are constant values. Hence, for a fixed value of exterior spacing $(s)$, the design parameters $(t, a$, and $\phi)$ can be calculated by using the "OC nomograph", in such way that the "capacity design" criteria are satisfied:

$$
\frac{\bar{H}_{C, s, k}}{\gamma_{o v, s}} \geq \bar{H}_{C, f, d} \quad \frac{\bar{H}_{C, h a, k}}{\gamma_{o v, h a}} \geq \bar{H}_{C, f, d} \quad \frac{\bar{H}_{C, s a, k}}{\gamma_{o v, s a}} \geq \bar{H}_{C, f, d}
$$

where: $\bar{H}_{C, s, k}, \bar{H}_{C, h a, k}$, and $\bar{H}_{C, s a, k}$ are the wall shear characteristic strengths per wall unit length corresponding to the resistances of studs, hold-down anchors and shear anchors, respectively. 
$\bar{H}_{C, f, d}$ is the wall shear design strength per wall unit length corresponding to the resistance of sheathing fasteners. Finally, $\gamma_{o v, s}, \gamma_{o v, h a}, \gamma_{o v, s a}$ are the overstrength factors of studs, hold-down anchors, and shear anchors, respectively.

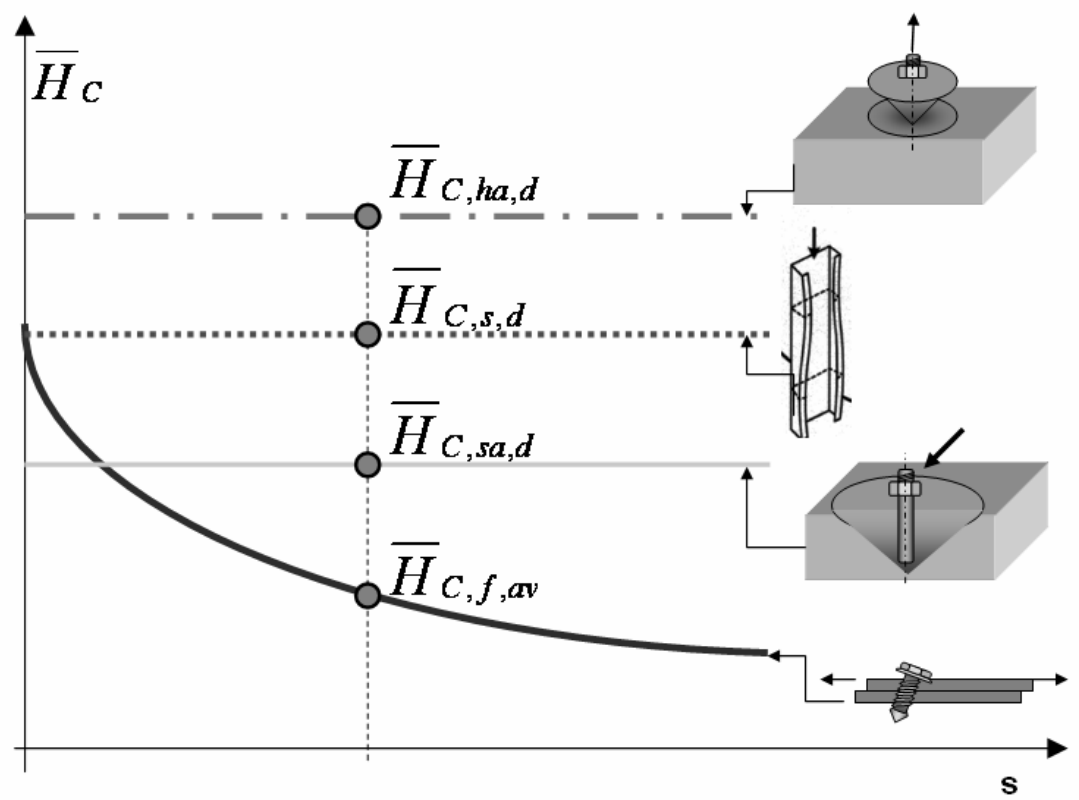

Figure 6. "OC" Nomograph

\section{DEFINITION OF STIFFNESS, STRENGTH, YIELDING AND ULTIMATE DISPLACEMENTS}

For the evaluation of the seismic response of this type of structures, according to the proposed design procedure, the relationships defining the wall shear response $\left(f_{k}, f_{H}, f_{y}\right.$, and $\left.f_{u}\right)$ as function of exterior spacing $(s)$ are required. In particular, in the case of LD seismic analysis, only wall stiffness $\left(f_{K}\right)$ and strength $\left(f_{H}\right)$ must be defined, while for the application of NS procedure, the definition of the elastic-plastic curve through the functions $f_{H}, f_{y}$, and $f_{u}$ usually requires the knowledge of the whole force $(H)$ - displacement $(d)$ response curve of the wall.

Different approaches can be used to perform an analysis devoted to obtain the functions $f_{K}, f_{H}, f_{y}$, and $f_{u}$; namely experimental (Serrette et al. [11]), numerical (Dolan [12], Dolan and Foschi [13], White and Dolan [14]) and analytical methodologies (Easley et al. [15], McCutchenon [16], Kaellsner and Lam [17], Hieta and Kesti [18]). In particular, analytical approaches used to analyze sheathed CFS shear walls generally allow only stiffness, strength and deflection $\left(f_{K}, f_{H}, f_{y}\right)$ to be predicted, without providing a reliable evaluation of the whole force - displacement response curve. To overcome this limitation, a specific analytical-numerical method has been developed by the authors allowing the prediction of the whole curve in terms of $f_{K}, f_{H}, f_{y}$ and $f_{u}$.

According to this methodology, the lateral displacement of a sheathed CFS shear wall can be obtained by adding four different deformation contributions (Figure 7):

$d=d_{f}+d_{s}+d_{b}+d_{a}$

where: $d_{f}$ represents the sheathing fasteners deformation, $d_{s}$ the sheathing panels shear deformation, $d_{b}$ the bending deformation, and $d_{a}$ the overturning deformation. 
If the local behaviour of sheathing fasteners governs the global lateral response of the wall (this is the usual condition), in the Eq. (11) the sheathing panels shear deformation, bending deformation and overturning deformation produce lateral displacements smaller than that due to sheathing fasteners deformation $\left(d_{s}<<d_{f}, d_{b}<<d_{f}, d_{a}<<d_{f}\right)$. In addition, $d_{s}, d_{b}$ and $d_{a}$ can be assumed as linear functions of the horizontal external force $(H)$, while the nonlinear lateral response of the wall is essentially the result of the inelastic behaviour of the sheathing fasteners $\left(d_{f}\right.$ is a nonlinear function of $H$ ). The model assumes that, at the beginning, the different deformation contributions can be independently evaluated and then they can be added.
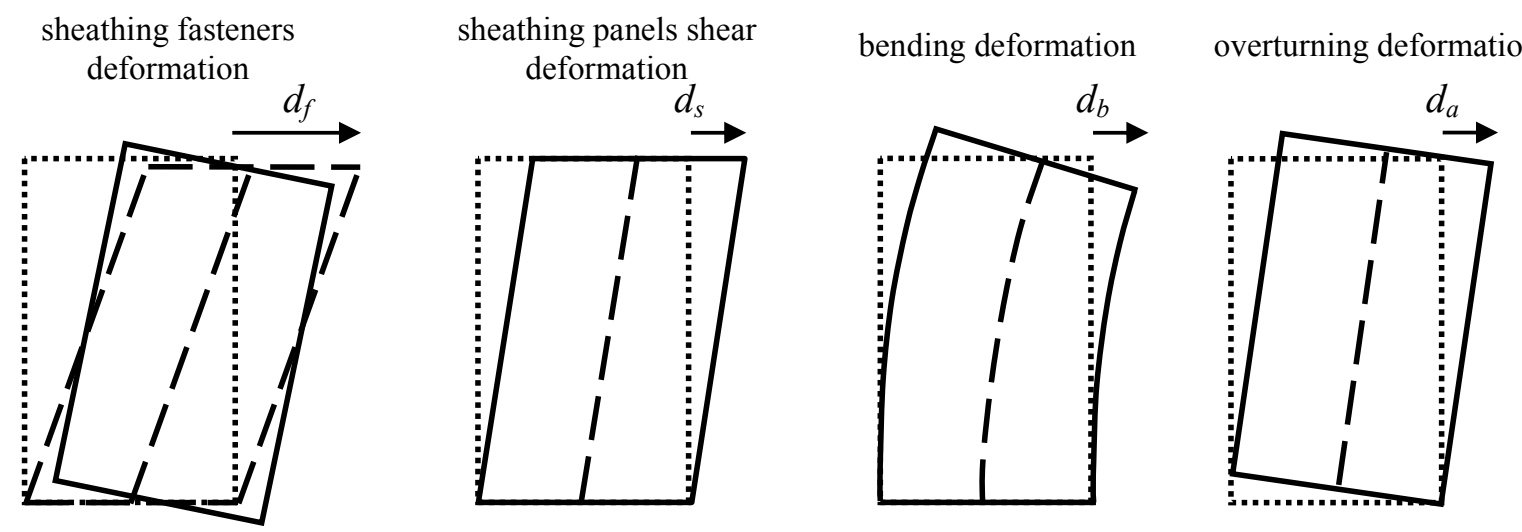

Figure 7. Deformation Component of a Sheathed CFS Shear Wall

For evaluating the lateral displacement $\left(d_{f}\right)$ due to the sheathing fasteners (frame-to-sheathing panel deformation), the following hypotheses are made: (a) the local failure of sheathing-to-frame connections governs the global collapse mode; (b) steel members are rigid and hinged to each other; (c) the frame deforms into a parallelogram and the relative frame-to-panel displacements are determined taking into account a rigid body rotation of panels; (d) the edges of the panel are free to rotate without interference from any other elements; (e) the wall is fully anchored; (f) relative displacements between the sheathing and frame are small compared with the panel size; (g) only shear deformation of sheathings is considered; (h) load-displacement curves of the sheathing-to-frame connections are schematized by using the relationship proposed by Richard and Abbott [19]. The assumed deformation of a sheathed cold-formed shear wall is shown in Figure 8.

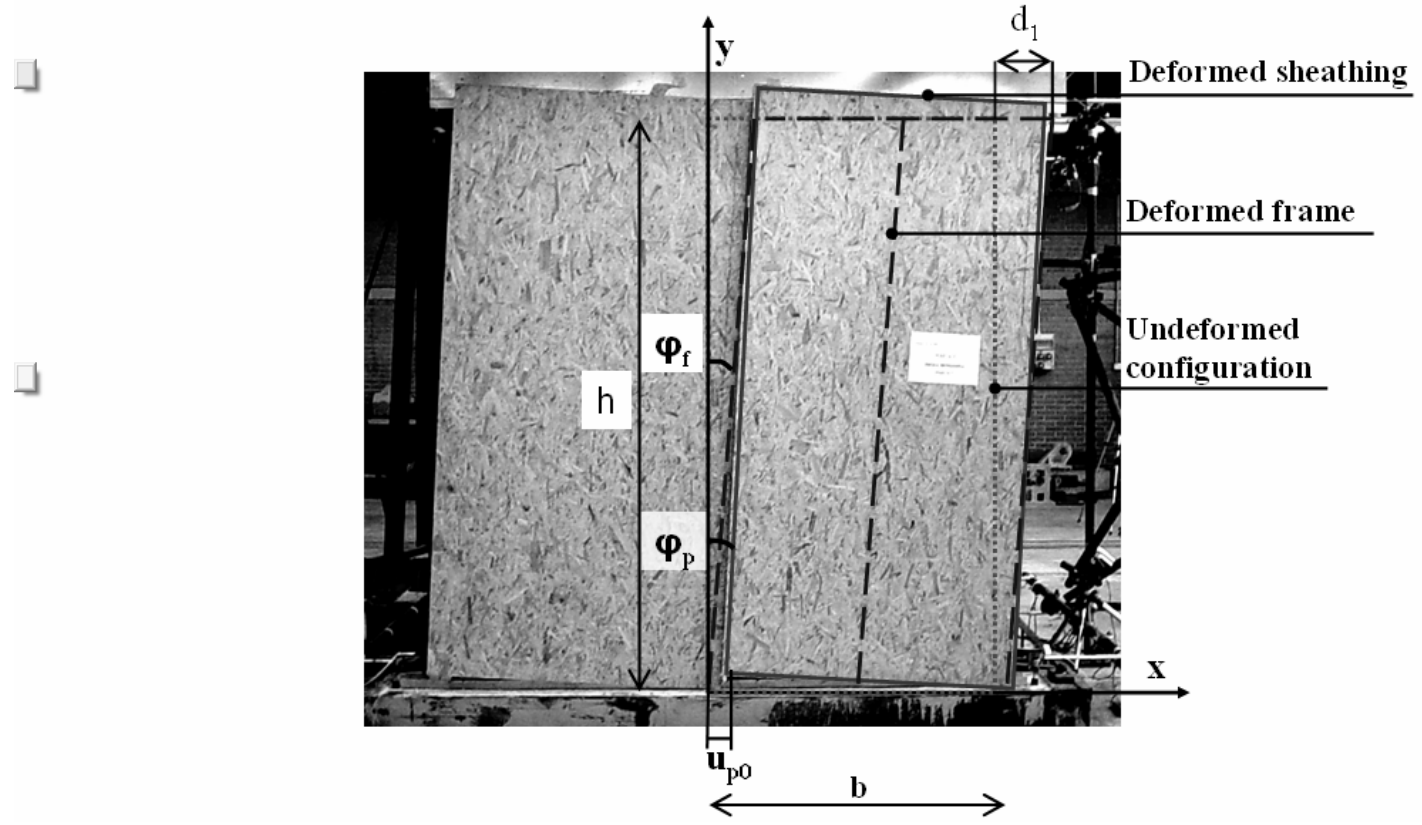

Figure 8. Full Scale Specimen and Assumed Deformation of a Shear Wall Specimen 
From equilibrium considerations, involving moment and horizontal force equilibrium for the panel and horizontal force equilibrium for the top track, and assuming that the load-deflection curve of sheathing-to-frame connections is linear, the following relationships can be obtained:

$\varphi_{f}=\frac{2 h\left[K_{x} \cdot I_{x}-\left(S_{x}\right)^{2}-\frac{b \cdot K_{x} \cdot S_{y}}{2}+K_{x} \cdot I_{y}\right] H}{\left(S_{x, m} \cdot S_{x}-I_{x, m} \cdot K_{x}+S_{e} \cdot S_{x}-I_{e} \cdot K_{x}\right)\left(2 I_{x}-b \cdot S_{y}\right)}$

$\varphi_{f}=\frac{-2 h\left[K_{x} \cdot I_{x}-\left(S_{x}\right)^{2}\right] H}{\left[\left(I_{x, m}+I_{e}\right) K_{x}-\left(S_{x, m}+S_{e}\right) S_{x}\right]\left(2 I_{x}-b \cdot S_{y}\right)}$

$u_{p 0}=\frac{h \cdot S_{x} \cdot H}{\left[I_{x, m}+I_{e}\right] K_{x}-\left[S_{x, m}+S_{e}\right] S_{x}}$

where:

$$
\begin{array}{lcc}
K_{x}=\sum_{i=1}^{n} k_{x, i} & S_{x}=\sum_{i=1}^{n} k_{x, i} \cdot y_{i} & I_{x}=\sum_{i=1}^{n} k_{x, i}\left(y_{i}\right)^{2} \\
S_{y}=\sum_{i=1}^{n} k_{y, i} \cdot x_{i} & I_{y}=\sum_{i=1}^{n} k_{y, i}\left(x_{i}\right)^{2} & S_{x, m}=\sum_{i=1}^{m} k_{x, i} \cdot y_{i} \\
K_{e}=k_{x e} \cdot n_{e} & S_{e}=k_{x e} \cdot n_{e} \cdot h & I_{e}=k_{x e} \cdot n_{e} \cdot h^{2}
\end{array}
$$

in which $\varphi_{f}$ and $\varphi_{p}$ are the rotations (defined positive as anticlockwise rotations) of the frame and sheathing panel, respectively; $u_{p 0}$ is the translation of the sheathing panel along the X direction; $k_{x, i}$ and $k_{y i}$ represent the stiffness of sheathing-to-frame connections for displacements along the $\mathrm{X}$ and $\mathrm{Y}$ directions, respectively; $k_{x e}$ is the stiffness of sheathing fasteners connecting the sheathing panel to the top track for displacement along the $\mathrm{X}$ direction; $x_{i}$ and $y_{i}$ are the connection coordinates along the $\mathrm{X}$ and $\mathrm{Y}$ directions; $i$ is the generic sheathing-to-frame connection, $n$ is the total number of sheathing-to-frame connections; $m$ is the number of fasteners connecting the sheathing panel to studs; $n_{e}$ is the number of fasteners connecting the sheathing panel to the top track; $H$ is the horizontal external force per unit length. When for sheathing fasteners a linear load-displacement response is assumed ( $k_{x, i}, k_{y, i}$, and $k_{x e}$ are constant values), Eq. 12 gives a closed-form solution and the top wall displacement $\left(d_{f}\right)$ can be evaluated as follows:

$$
d_{f}=\varphi_{f} h
$$

where $\varphi_{f}$ is calculated through Eq. 12. When a nonlinear load-displacement curve is adopted for sheathing fasteners, Eq. 12 through 15 can be written in differential format and can be used in a numerical step-by-step procedure which allows the load $(H)$ vs. deflection $\left(d_{f}\right)$ response curve of the wall to be obtained. More details about the numerical procedure are given in Fiorino et al. [20]. The lateral displacement due to the shear deformation of the sheathing panels $\left(d_{s}\right)$ is obtained by adopting the equation for shear deformation of a thin, edge-loaded, plate:

$$
d_{s}=\frac{H \cdot h}{G \cdot t_{p} \cdot b}
$$


where: $G$ is the shear modulus of elasticity of the panel material; $b$ is the width of the sheathing panel; $t_{p}$ is the panel thickness.

The bending displacement $\left(d_{b}\right)$ may be evaluated by considering the wall as a cantilever having a cross-section made of the only end studs:

$d_{b}=\frac{2 H \cdot h^{3}}{3 E \cdot A \cdot l^{2}}$

where: $E$ is the Young's modulus of steel; $A$ is the gross cross-sectional area of an end stud.

The lateral displacement due to overturning $\left(d_{a}\right)$ is calculated from the following equation:

$d_{a}=\frac{H \cdot h^{2}}{l^{2} \cdot K_{a}}$

where $K_{a}$ is the axial stiffness of the hold-down device, which can be obtained from hold-down manufacturers. Typical values of stiffness $K_{a}$ have been obtained by considering the Simpson metal-to-metal connectors (Simpson Strong-Tie Company [21]) ranging from 27 to $31 \mathrm{kN} / \mathrm{mm}$ for S/HD hold-down connectors.

\section{APPLICATION OF NOMOGRAPHS: A CASE STUDY}

The house presented hereafter shows the architectural and technological possibility of a stick built construction and, mainly, it has been designed in order to study the applicability of the presented nomographs. The architecture has been designed without having in mind any specific location or particular inhabitant requirements. It aims to be a prototype of a contemporary housing in which flexibility, environmental comfort and high structural performance are the keywords.

The dwelling develops in two floors and is organized in wide and regular spaces where the walls are distributed along the perimeter and in the center of house, in order to assure the maximum flexibility of the internal spaces (Figure 9).

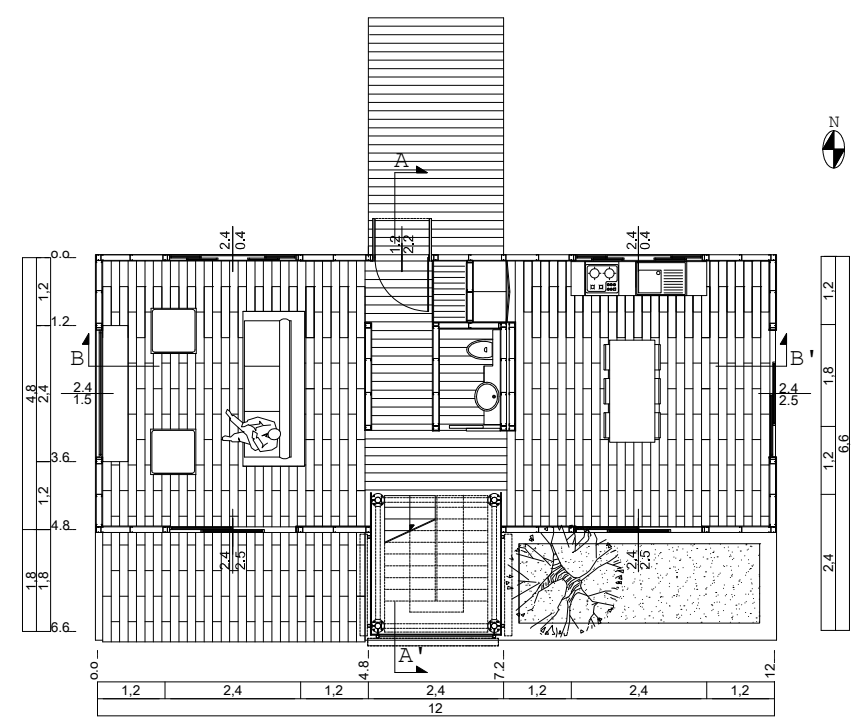

Figure 9. Case Study: Ground Floor 
The opening distribution follows environment requirements, introducing thin openings on the Nord front and wide and adequately shielded openings on the South and South-West elevations (Figure $10)$.
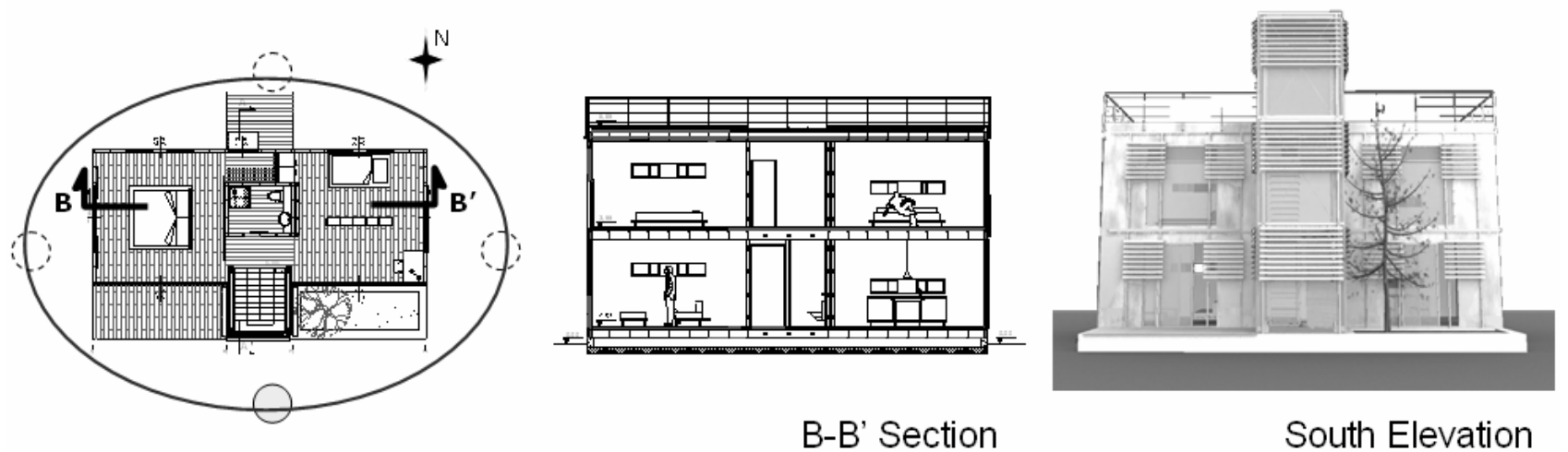

Figure 10. Case Study: Opening Distribution

The construction arises on a concrete slab, on which the steel frame is anchored by clip angles and post installed anchors. The basic framing system is characterized by discontinued walls, interrupted at each floor level by floor framing. The floor framing is composed by joists, horizontal bearing members, spaced at $600 \mathrm{~mm}$ and aligned with vertical load bearing framing members. Joists are connected at each end by a floor track: an horizontal member used as band or rim joists for flooring systems. Web stiffeners are installed at each joist bearing location to strengthen the member against web crippling and to assure the continuity of vertical bearing members (Figure 11).

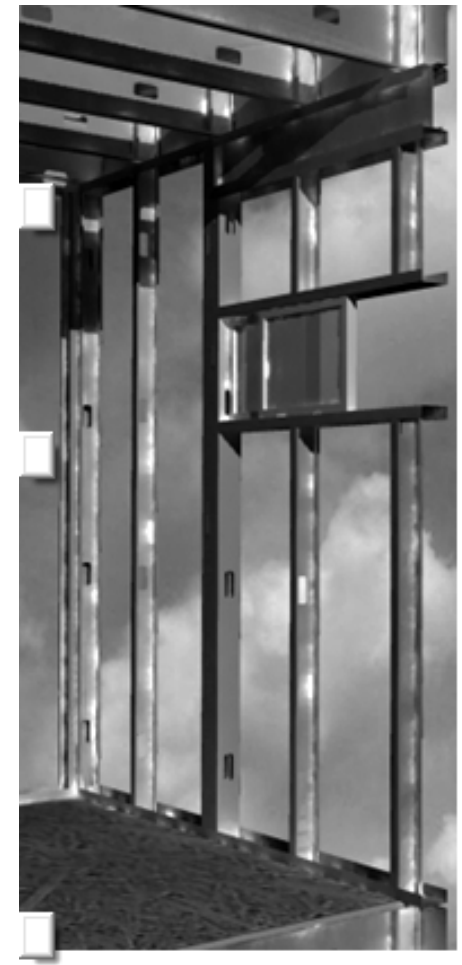

b) Wall framing

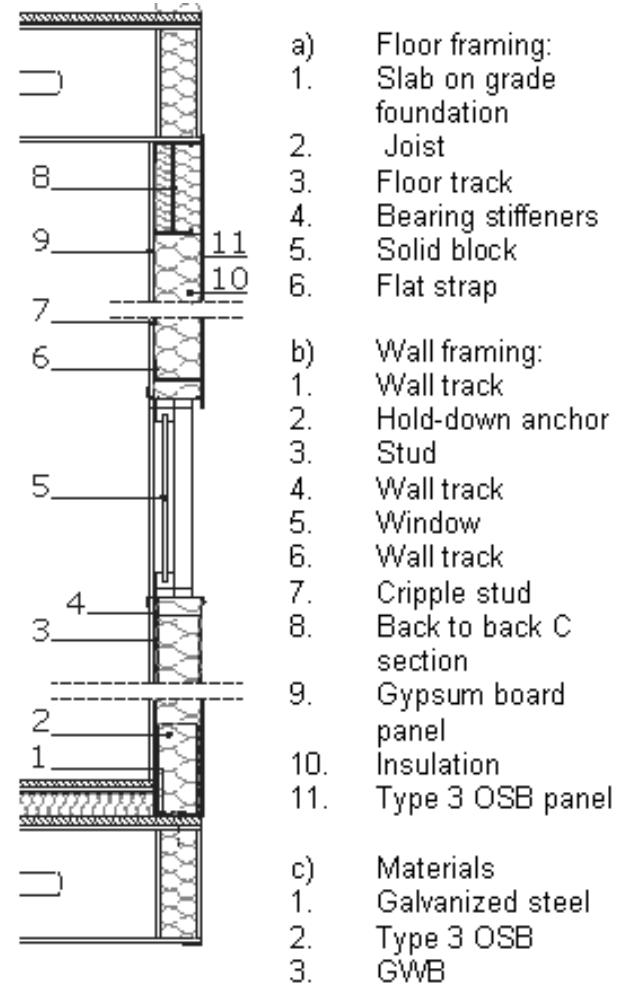

GWB

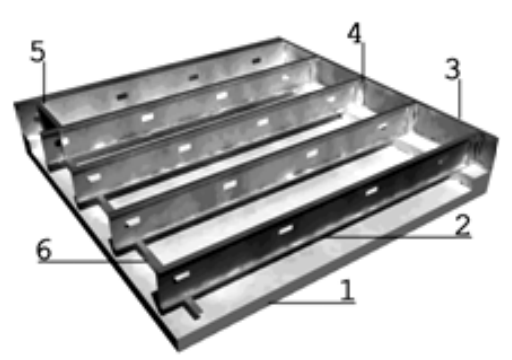

a) Floor framing

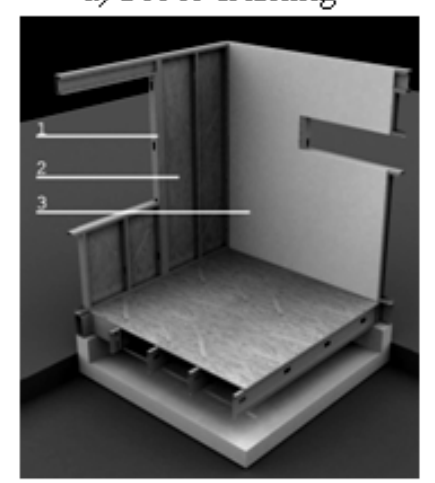

c) Materials

Figure 11. Case Study: Components and Materials

Hence, the walls have been designed following the proposed procedure.

As first step, according to the proposed design process (phase 1), the following "assigned" design parameters have been adopted: 
- $\quad 2700 \mathrm{~mm}$ high $(h)$ and $1200 \mathrm{~mm}$ long $(l)$ wall with stud spaced $(c)$ at $600 \mathrm{~mm}$;

- $\quad 9.0 \mathrm{~mm}$ thick Type 3 OSB external panels and $12.5 \mathrm{~mm}$ thick standard GWB internal panels, both having vertical orientation;

- $\quad 4.2 \times 25 \mathrm{~mm}($ diameter $\times$ length) flat head self-drilling screw sheathing fasteners having interior spacing $(p)$ of $300 \mathrm{~mm}$ and edge distance $(e)$ of $10 \mathrm{~mm}$;

- studs having $100 \times 50 \times 10 \mathrm{~mm}$ (outside-to-outside web depth $\times$ outside-to-outside flange size $\times$ outside-to-outside lip size) lipped channel section, and track having $100 \times 40 \times 1.00 \mathrm{~mm}$ (outside-to-outside web depth $\times$ outside-to-outside flange size $\times$ thickness) unlipped channel section, both fabricated from $\mathrm{S} 350 \mathrm{GD}+\mathrm{Z}$ hot dipped galvanized zinc coated steel grade;

- $\quad$ S/HD10B metal-to-metal connectors by Simpson Strong-Tie Company (Simpson Strong-Tie Company [21]) and HIT-RE 500 with HIS-N 8.8 adhesive-bonded anchors by HILTI (Hilti [22]) hold-down devices;

- $\quad$ HST M8 mechanical shear anchors by HILTI (Hilti [22]).

The evaluation of the sheathing fasteners exterior spacing (phase 2) performed with nomographs for LD and NS seismic analysis are shown in Figures 12 and 13, respectively. In particular, in the "LD" nomograph both strength $\left(\bar{H}_{C}\right)$ and action $\left(\bar{H}_{D}\right)$ are expressed for wall unit length and have been normalised respect to the seismic weight $(w)$. In the "NS" nomograph the "strength" $\left(\bar{H}_{C} \cdot g / w\right)$ and "action" $\left(S_{a}\right)$ parameters have been normalised respect to the gravity acceleration $\left(\bar{H}_{C} / w, S_{a} / g=\bar{H}_{D} / w\right)$.

The functions $f_{K}, f_{H}, f_{y}$, and $f_{u}$ used in these nomographs have been obtained by applying the model proposed in Section 3 starting from force - displacement (slip) sheathing fastener response curve derived on the basis of experimental tests carried out by the authors on typical screw connections for cold-formed steel housing (Fiorino et al. [23]).

The elastic acceleration demand spectra provided by Eurocode 8 (CEN [24]) and adopted also by the new Italian Seismic Code (OPCM [25]) have been assumed as spectral acceleration functions $\left(f_{\text {SaT }}, f_{\text {Sad }}\right)$. In particular, the Italian Seismic Code defines soil types A, B, C, D, and E according to the Eurocode 8 classification, but only three spectra are adopted, by grouping the soil types B, C and $\mathrm{E}$ under one spectrum type ("BCE"). Values of the peak design ground acceleration $\left(a_{g}\right)$ on type A soil (for earthquakes having 10\% chance of being exceeded over 50 years - average return period 474 years) equal to $0.35 \mathrm{~g}, 0.25 \mathrm{~g}$, and $0.15 \mathrm{~g}$ (for seismic zones having low, medium and high intensity, respectively) have been assumed. In addition, the spectra adopted for the LD procedure have been obtained by assuming a strength reduction factor $q=4.0$ (Boudreault et al. [26]). A viscous damping ratio $\xi=5 \%$ has been adopted in all cases, in accordance with literature data (Dubina et al. [27], The Committee on Light-gauge Steel Structures [28]).

In Figure 12, the LD seismic analysis is performed for the presented case study, which is characterized by a seismic weight equal to $w=20 \mathrm{kN} / \mathrm{m}$. Moreover, a peak design ground acceleration equal to $a_{g}=0.35 g$ and soil type "B" have been considered. By assuming as attempt an exterior spacing $s=150 \mathrm{~mm}$, for a seismic weight $w=20 \mathrm{kN} / \mathrm{m}$, the point A on the top left graph identifies the normalised strength $\left(\bar{H}_{C} / w=0.40\right)$, while the point $\mathrm{B}$ on the bottom left graph defines the stiffness $(\bar{K}=0.95 \mathrm{kN} / \mathrm{mm} \cdot \mathrm{m})$. The point $\mathrm{C}$ (defining a structural period $T=0.292 \mathrm{~s}$ ) is obtained on the bottom right graph by the intersection of the horizontal line drawn from point $\mathrm{B}$ and the curve relevant to $w=20 \mathrm{kN} / \mathrm{m}$. Finally, on the top right graph the intersection of the vertical line drawn from point $\mathrm{C}$ and the curve relevant to $a_{g}=0.35 g$ and "B" soil type defines the normalised actions $\left(\bar{H}_{D} / w=0.27\right.$, point D). Since acting seismic force is less than seismic resistance (point $\mathrm{D}$ is lower than point $\mathrm{A})$, then the assumed exterior spacing $(s=150 \mathrm{~mm})$ is adequate $\left(\bar{H}_{D} / \bar{H}_{C}=0.68\right)$. 
Hence, a NS seismic analysis has been performed (Figure 13). In this case, starting from $s=150 \mathrm{~mm}$ and $w=20 \mathrm{kN} / \mathrm{m}$ the point $\mathrm{A}$ on the top left graph defines the normalised strength $\left(\bar{H}_{C} / w=0.40\right)$, while the points $\mathrm{B}$ and $\mathrm{C}$ on the bottom right graph identify the yield displacement $\left(d_{y}=9 \mathrm{~mm}\right)$ and ultimate displacement $\left(d_{u}=d_{C}=52 \mathrm{~mm}\right)$. Therefore, the capacity spectrum (broken line O-Y-U) is obtained on the top right graph by the intersection of the horizontal line drawn from point $A$ and vertical lines drawn from points B and C. Finally, by considering on the top right graph the relevant demand spectrum ( $a_{g}=0.35 g$ and "B" soil type), the well known capacity-demand-diagram method (ATC-40 [29]) can be used to find the displacement demand $\left(d_{D}=34 \mathrm{~mm}\right.$, point D). The NS procedure confirms that the assumed exterior spacing is adequate $\left(d_{D} / d_{C}=0.65\right)$.

The last step of the proposed design process (phase 3) is performed through the "OC" nomograph (Figure 14). This nomograph shows the design resistance of sheathing fasteners $\left(\bar{H}_{C, f, d}\right)$ together with the characteristics resistances of studs, hold-down anchors and shear anchors reduced by considering the relevant overstrength factors $\left(\bar{H}_{C, s, k} / \gamma_{o v, s}, \bar{H}_{C, h a, k} / \gamma_{o v, h a}, \bar{H}_{C, s a, k} / \gamma_{o v, s a}\right)$. In particular, by assuming: $\gamma_{o v, s}=2.0, \gamma_{o v, h a}=4.0, \gamma_{o v, s a}=3.0$, and $\bar{H}_{C, s, k} / \bar{H}_{C, s, d}=1.0$, $\bar{H}_{C, h a, k} / \bar{H}_{C, h a, d}=2.0, \bar{H}_{C, s a, k} / \bar{H}_{C, s a, d}=1.5$, and $\bar{H}_{C, f, a v} / \bar{H}_{C, f, d}=2.0$, different values of $\bar{H}_{C, s, k} / \gamma_{o v, s}, \bar{H}_{C, h a, k} / \gamma_{o v, h a}, \bar{H}_{C, s a, k} / \gamma_{o v, s a}$ have been calculated by varying the wall component design parameters $t, a$, and $\phi$, respectively. In this way the "capacity design" criteria (see Eq. 10) can be applied for the selection of more convenient values of such parameters. In the examined case study this means to draw a vertical line corresponding to the selected exterior spacing $s=$ $150 \mathrm{~mm}$ on the "OC" nomograph and then to identify the point corresponding to the resistance of sheathing fasteners (point A, $\bar{H}_{C, f, d}=12 \mathrm{kN} / \mathrm{m}$ ). The definition of $t, a$, and $\phi$ is then performed by identifying, for each component, the relevant line which is immediately higher than the point $\mathrm{A}$ ( $t=1.0 \mathrm{~mm}$, point $\mathrm{L} ; a=300 \mathrm{~mm}$, point $\mathrm{G}, \phi=10 \mathrm{~mm}$, point $\mathrm{H})$.

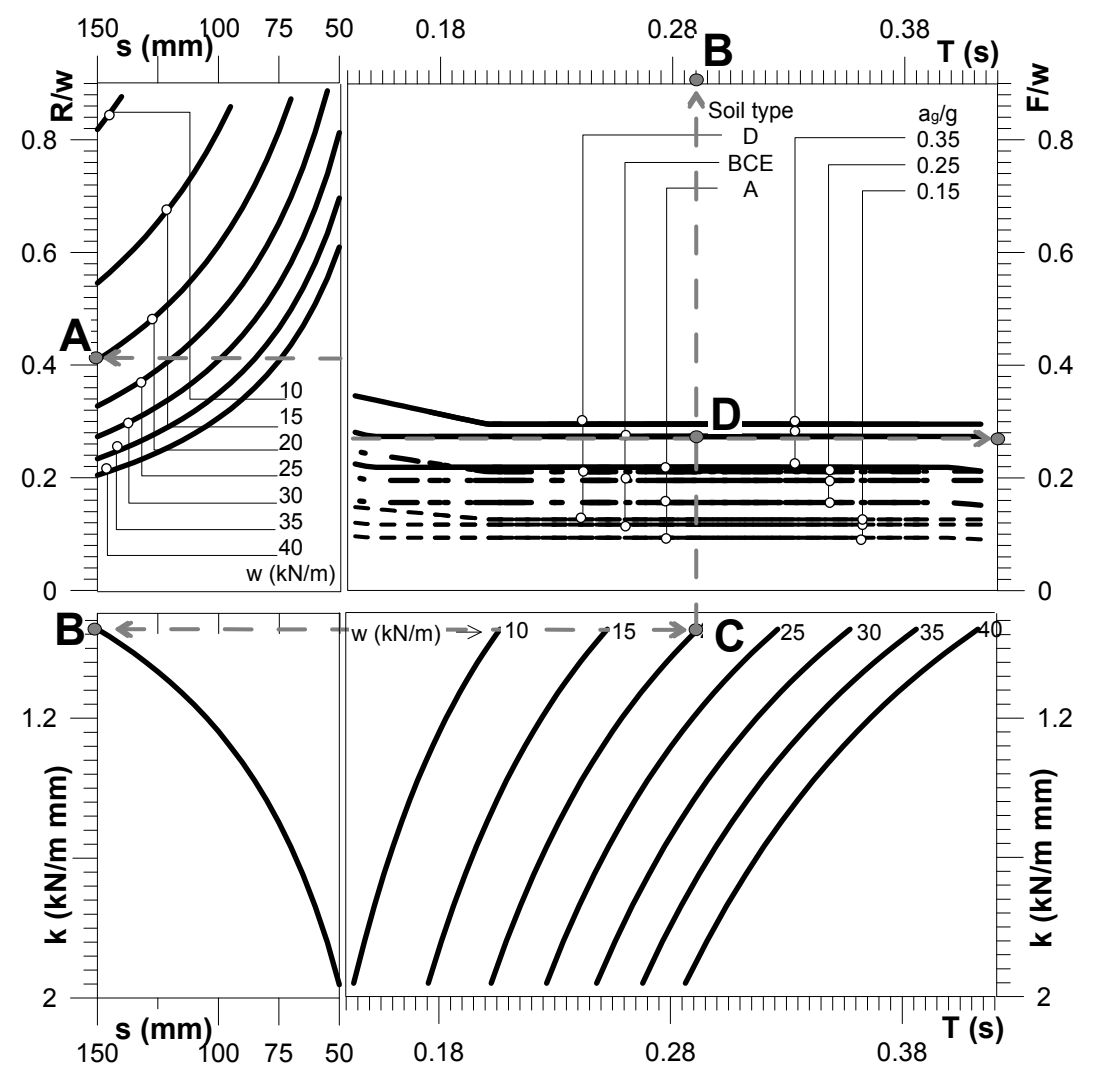

Figure 12. Application of "LD” Nomograph 


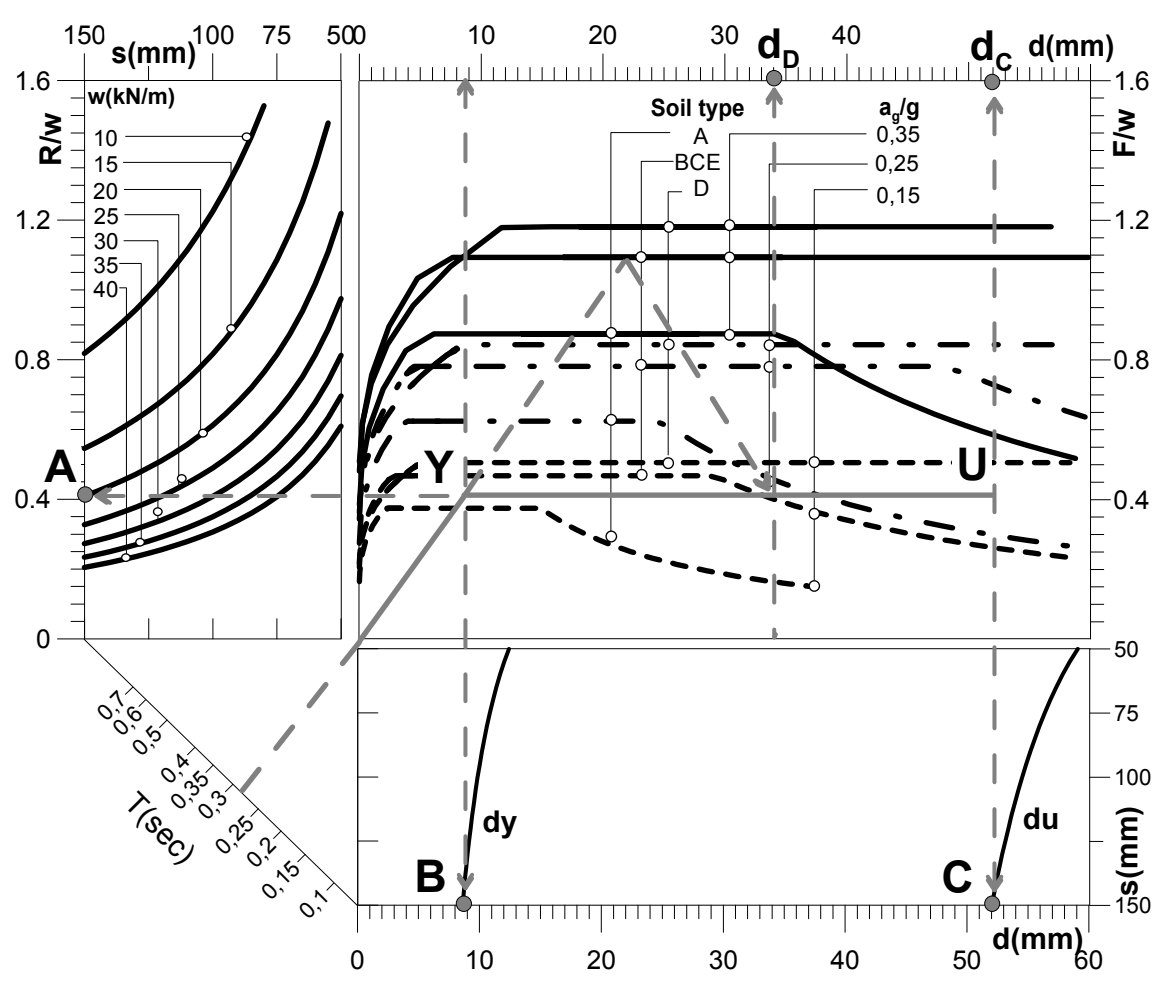

Figure 13. Application of "NS" Nomograph

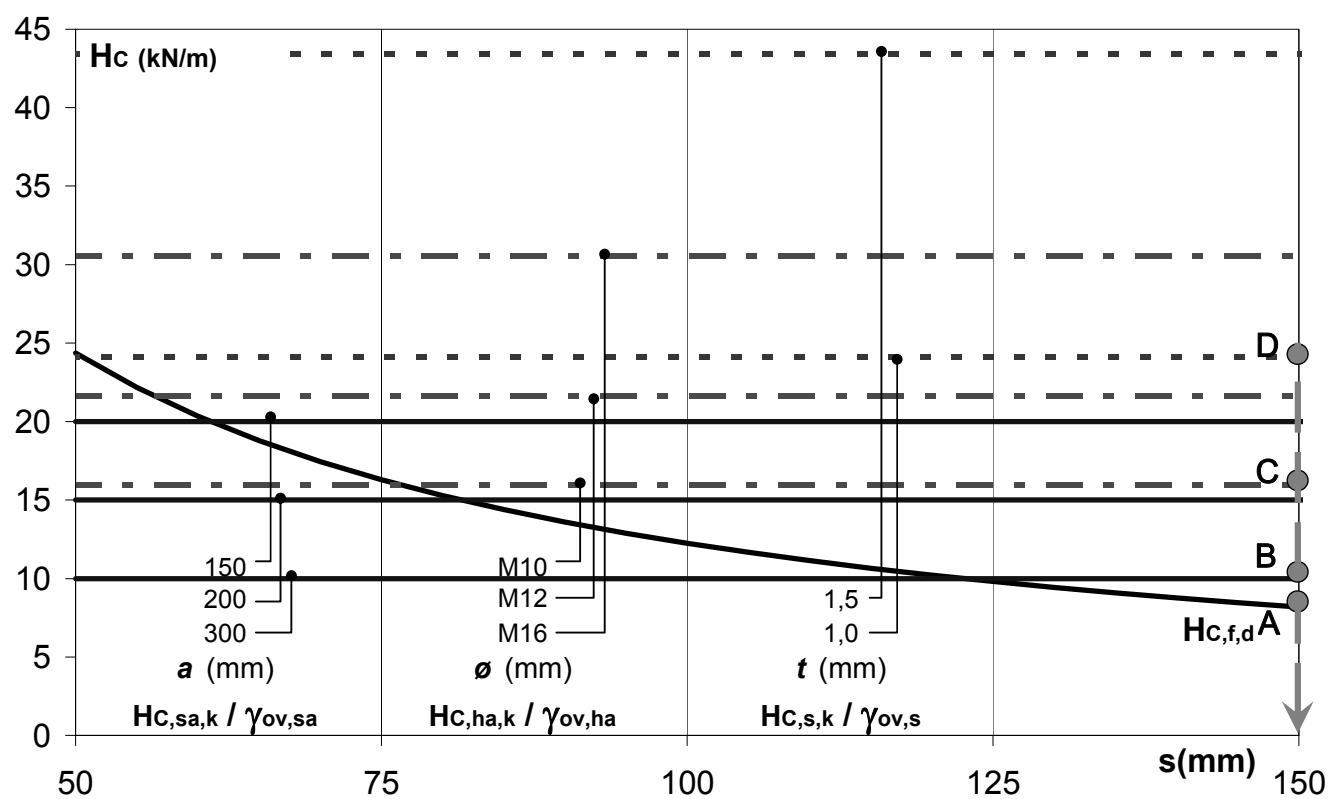

Figure 14: Application of "OC" Nomograph

\section{CONCLUSIONS}

This paper presents a design procedure for cold formed steel housing in seismic area. It is based on the recognized assumption that the shear walls are the main resistant building components and that the best behaviour of a CFS housing can be reached when the collapse under seismic loads is due to the sheathing-to-stud screw failure. Hence, the proposed design procedure, after the definition of the shear wall geometry and technological components, allows the screw sheathing-to-stud fasteners spacing to be defined through a linear dynamic (LD nomograph) analysis or a nonlinear static (NS nomograph) analysis. Finally, the complete definition of all the other wall structural 
components (studs, shear and hold down anchors) is performed in accordance with "capacity design criteria" (OC nomograph). Moreover, the analytical model used to define the wall strength curves is presented. Finally the procedure applicability is investigated through the design of a two-story CFS dwelling.

\section{REFERENCES}

[1] Gad, E. F., Chandler, A. M., Duffield, C. F., and Stark, G., "Lateral Behaviour of Plasterboard-Clad Residential Steel Frames.” J. Struct. Eng., 1999, Vol. 125, pp. 32-39.

[2] Tian, Y.S., Wang, J. and Lu, T.J. "Racking Strength and Stiffness of Cold-formed Steel Wall Frames", Journal of Constructional Steel Research, 2004, Vol. 60, pp. 1069-1093.

[3] Branston, A.E., Chen, C.Y., Boudreault, F.A. and Rogers, C.A., "Testing of Light-gauge Steel-frame - Wood Structural Panel Shear Walls," Can. J. Civ. Eng., 2006, Vol. 33, pp. 561-572.

[4] Fülöp, L.A. and Dubina, D., "Design Criteria for Seam and Sheeting-to-Framing Connections of Cold-Formed Steel Shear Panels", Journal of Structural Engineering, ASCE, 2006, Vol.132, No.4, pp. 582-590.

[5] Landolfo, R., Fiorino, L. and Della Corte, G., "Seismic Behaviour of Sheathed Cold-formed Structures: Physical Tests," Journal of Structural Engineering, ASCE, 2006, Vol. 132, No.4, pp 570-581.

[6] Langea, J. and Naujoksb, B. "Behaviour of Cold-formed Steel Shear Walls Under Horizontal and Vertical Loads", Thin-Walled Structures, 2006, Vol. 44, pp. 1214-1222.

[7] Serrette, R, Nguyen, H. and Hall, G., "Shear Wall Values for Light Weight Steel Framing", Report No. LGSRG-3-96, Light Gauge Steel Research Group, Department of Civil Engineering, Santa Clara University, Santa Clara, 1996.

[8] Uniform Building Code, "International Conference of Building Officials", Vol. 2, Whittier, CA, USA, 1997.

[9] International Building Code, "International Code Council", Inc. Falls Church, VA, USA, 2000.

[10] Fiorino, L., Iuorio, O., Landolfo, R., "Sheathed cold-formed steel housing: a seismic design procedure", Thin Walled Structures, 2009, Vol. 47, pp. 919 - 930.

[11] Serrette, R., Hall, G. and Nguyen, H., "Dynamic Performance of Light Gauge Steel Framed Shear Walls", Proceedings of 13th International Specialty Conference on Cold-formed Steel Structures, St. Louis, 1996, pp. 487-498.

[12] Dolan, J.D., "The Dynamic Response of Timber Shear Walls", PhD thesis, University of British Columbia, Vancouver, 1989.

[13] Dolan, J.D. and Foschi, R.O., "Structural Analysis Model for Static Loads on Timber Shear Walls", Journal of Structural Engineering, 1991, Vol. 117, No. 3, pp. 851-861.

[14] White, M.W. and Dolan, J.D., "Nonlinear Shear-Wall Analysis", Journal of Structural Engineering, 1995, Vol. 121, No. 11, pp. 1629-1635.

[15] Easley, J.T., Foomani, M. and Dodds, R.H., "Formulas for Wood Shear Walls", Journal of Structural Division, 1982, Vol. 105, pp. 2460-2478.

[16] McCutchenon, W.J., "Racking Deformation in Wood Shear Walls", Journal of Structural Engineering, ASCE, 1985, Vol. 111, pp. 257-269.

[17] Kaellsner, B. and Lam, F., "Diaphragms and Shear Walls". In STEP Lectures: Holzbauwerke nach Eurocode 5-Grundlagen, Entwicklungen, Ergaenzungen, Fachverlag Holz, Duesseldorf, Germany: 15/1-15/19, 1995.

[18] Hieta, J. and Kesti, J., "Design Recommendations for Shear Walls Braced with Sheathings", Teräsrakenteiden tutkimus- ja kehityspäivät 13.-14.6.2002, Mikkeeli, Finnish Constructional Steelwork association, 2002. 
[19] Richard, R.M. and Abbott, B.J., "Versatile Elastic-plastic Stress-strain Formula", Journal of Mechanical Engineering, 1975, Vol. 101, No. 4, pp. 511-515.

[20] Fiorino, L., Della Corte, G. and Landolfo, R., "Lateral Response of Sheathed Cold-Formed Shear Walls: An Analytical Approach", Proceedings of 18th International Specialty Conference on Cold-Formed Steel Structures, Orlando, 2006.

[21] Simpson Strong-Tie Company, http://www.strongtie.com, 2007.

[22] Hilti North America product technical guide, 2005.

[23] Fiorino, L., Della Corte, G. and Landolfo, R., "Experimental Tests on Typical Screw Connections for Cold-formed Steel Housing”, Engineering Structures, Elsevier, 2007, Vol. 29, No. 8, pp.1761-1773.

[24] EN 1998-1 - Eurocode 8 "Design of Structures for Earthquake Resistance - Part 1: General Rules, Seismic Actions and Rules for Buildings", European Committee for Standardization (CEN), Bruxelles, 2005.

[25] OPCM 3431/2005. "Primi Elementi in Materia di criteri Generali per la Classificazione Sismica del Territorio Nazionale e di Normative Tecniche per le Costruzioni in Zona Sismica", Ordinanza della Presidenza del Consiglio dei Ministri No.3431/2005, Rome, 2005.

[26] Boudreault, F.A., Blais, C. and Rogers, C.A., "Seismic Force Modification Factors for Light-gauge Steel-frame - Wood Structural Panel Shear Walls", Canadian Journal of Civil Engineering, 2007, Vol. 34, pp. 56-65.

[27] Dubina, D., Fülöp, L.A., Aldea, A., Demetriu, S. and Nagy, Zs., "Seismic Performance of Cold-formed Steel Framed Houses", Proceedings of 5th International Conf. on Behaviour of Steel Structures in Seismic Areas (STESSA), Yo1kohama, 2006, pp 429-435.

[28] The Committee on Light-gauge Steel Structures, The Japan Iron and Steel Federation, Steel-Framed House Association. "Steel-framed Houses - High Structural Performances and Habitability, Steel Construction Today \& Tomorrow", 2004, Vol. 10, pp. 1-9.

[29] ATC 40 "Seismic Evaluation and Retrofit of Concrete Buildings", Applied Technology Council (ATC), Redwood City, 1996. 


\title{
INFLUENCE OF PARTIAL LOADING ON THE BEHAVIOUR OF PALLET RACK STRUCTURES
}

\author{
F. Al Qarud ${ }^{1}$, A. Shatnawi ${ }^{1}$, M.S. Abdel-Jaber ${ }^{1}$ and R.G. Beale, ${ }^{2, *}$ \\ ${ }^{1}$ Department of Civil Engineering, University of Jordan, Amman, Jordan \\ ${ }^{2}$ Department of Mechanical Engineering, Wheatley Campus \\ Oxford Brookes University, Oxford, OX33 1HX, UK \\ *(Corresponding author: E-mail: rgbeale@brooks.ac.uk)
}

Received: 11 June 2008; Revised: 19 September 2008; Accepted: 22 September 2008

\begin{abstract}
This paper presents the results of an investigation into the effects of partial loading on the behaviour of steel pallet racks. A six level, six bay, 3-dimensional pallet rack frame model was built using ABAQUS and a geometrically non-linear analysis conducted. Twelve different load combinations were analysed under different boundary conditions and different side imperfections. Most combinations failed by sway buckling. Depending upon the side imperfection and the base condition critical combinations involved having completely unloaded bays or lifts adjacent to two or more fully loaded bays or lifts with reductions in capacity over a fully loaded rack of up to $40 \%$.
\end{abstract}

Keywords: Pallet rack structures, load combinations, pattern loading, semi-rigid connections, non-linear analysis, storage structures

\section{INTRODUCTION}

\subsection{Steel Storage Pallet Rack Systems}

Steel storage pallet racks are widely used throughout the world for storing industrial goods. Moreover these structures provide high storage density. The goods to be stored are generally in cartons or boxes stored on pallets. A typical example is shown in Figure 1.

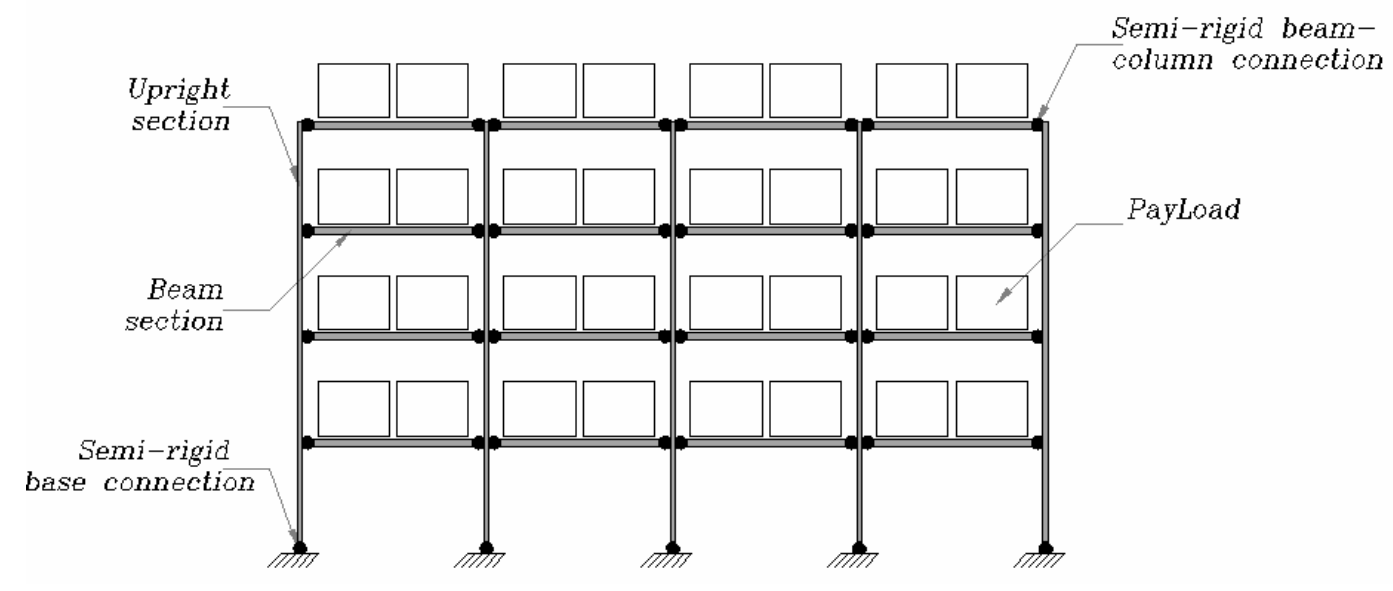

Figure 1. Pallet Rack Structure

Pallet racks are commonly made from cold roll formed steel. Such structures usually have a large number of bays and beam levels. The main components for racking system are vertical supports or uprights, beams, connections and bracing. Uprights are generally thin-walled, perforated open sections with low torsional stiffness. The beams are often thin-walled closed sections with high torsional stiffness. Bracing systems are generally placed in the cross aisle direction. The connections between beams and uprights are semi-rigid and are usually made by the use of tabs and lugs welded onto the ends of beams making a boltless connection into the perforations of the 
uprights [1]. Bolted connections are used to connect the upright with the ground. The base-plate connections can be considered either as semi-rigid or pinned although research by Beale and Godley [2] has shown that under certain combinations of side loads that pinned connections can carry higher loads. Godley [3] has also pointed out that under many load combinations that the difference in performance between semi-rigid and rigid bases can often be considered negligible and that in many cases bases can be considered as either pinned or fixed.

\subsection{Pattern Loading}

A lot of research has been reported on the subject of analyses and design of pallet rack structures, for example [4-9]. However few of these publications have reported the results of pattern loading as is often required by design codes such as Federation Europeene de la Manutention (FEM) code [10] and the Rack Manufacturers Institute (RMI) code [11]. According to the FEM code [10] rack structures may be analysed by considering both down and cross-aisle directions separately as two 2-dimensional frame structures. In addition some of the loads used in the analysis of the rack structure are dead load, imposed loads from stored materials and imperfections in the down and cross-aisle aisle directions. For the design of the upright it is required that both fully loaded and pattern loaded frames shall be shall be considered; these patterns loads are often fully loaded except that a load from a beam near the middle of the structure at the lowest level is removed [12].

Beale and Godley [12-13] developed a program to analyse and design regular pallet racks according to the FEM code. The program determines the buckling load of an equivalent free sway structure and using stability functions, calculates the axial and shear forces and the bending moments within the structure including the non-linear $(F-\Delta)$ effects. The results of the program showed that pattern load effects in combination with imperfections often dominate the failure loads of the analysed racks.

Morz et al [14] and Olsson and Sandberg [15] presented a numerical study with the aim of investigating the influence of removing components from pallet racks, such as horizontal beams or cross-aisle bracing members. Typical cases considered were removing all five pairs of beams at the lowest beam level or having two pairs of beam missing. The results showed that for all these cases of loading the total load-carrying capacity of frame was reduced and the pallet rack failed in a global down-aisle sway mode.

The research reported in this paper investigates the effects on the total load carrying capacity of the frame of different types of pattern loading in combination with different geometrical imperfections. In addition, this study compares frame response under different base conditions - semi-rigid, pinned and fixed.

\section{ANALYSES}

\subsection{Pallet Model}

Steel pallet rack structures are 3-dimensional frame systems. The performance of these structures with fixed-bases under 36-load combinations is compared with similar frames with pinned and semi-rigid bases. In the analyses conducted the vertical and side loads were increased proportionally to determine the maximum loads before failure and the maximum reactions and deflections recorded. The model pallet rack considered in this paper contained 6-levels and 6-bays, the height to first story was $400 \mathrm{~mm}$ as it is common for racking frames to have a low first story, the height of each subsequent story was $1400 \mathrm{~mm}$ and the beam lengths were $2400 \mathrm{~mm}$. Front 
and rear frames were connected by zig-zag bracing, with an initial horizontal member $100 \mathrm{~mm}$ above the ground and with each ' $\mathrm{zig}$ ' of height $850 \mathrm{~mm}$. A horizontal cross-aisle member was at top of the highest 'zig'. The model is shown in Figure 2.

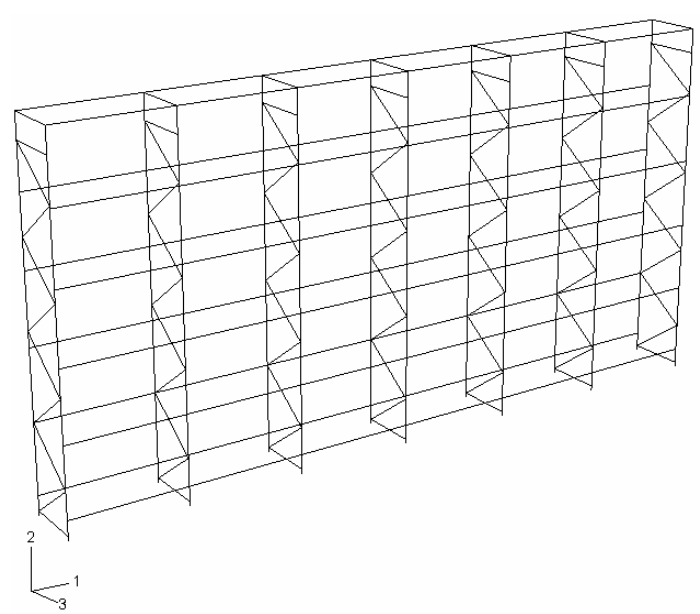

Figure 2. Isometric View of Model

\subsection{Cases of Loading}

36-combinations of loads were analysed and the maximum load that the structure carried determined, the choice of these types of loading was due to the eccentricities caused by imperfections. The cases of loading were considered in this paper are:

- Appling a UDL to all horizontal members (general case).

- Removing the loading on alternate bays for all lifts (case nos.1 and 2)

- Applying checker-board loading by removing loads on alternate bays and columns (case nos. 3 and 4).

- Loading two bays in three (case nos. 5 and 6).

- Removing the loading on alternate levels (case nos. 7 and 8)

- Removing the loading on one bay in the bottom two levels (case nos. 9-12)

- Appling an initial out-of-plumb due to frame imperfections, in down aisle and cross aisle directions.

Figure 3 shows all cases of loading used in the analysis:

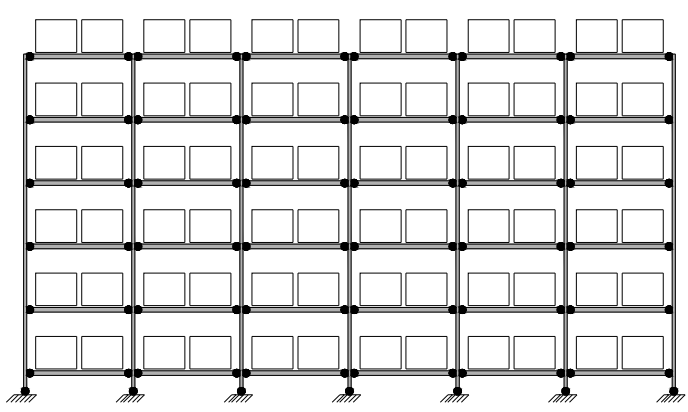

(a) General Case 


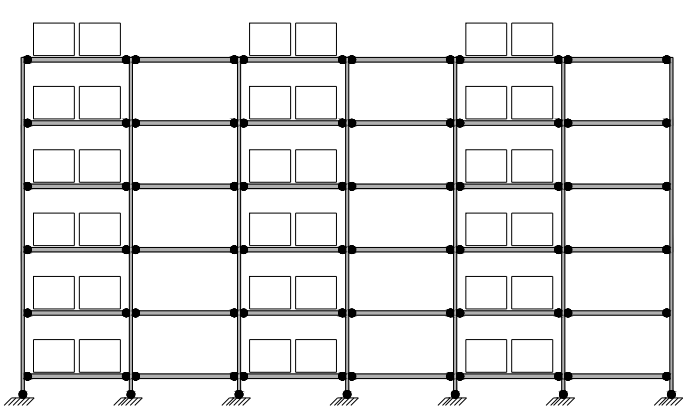

(b) Case 1

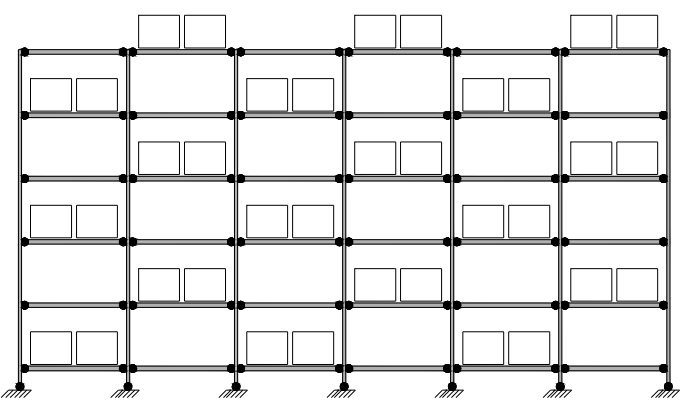

(d) Case 3

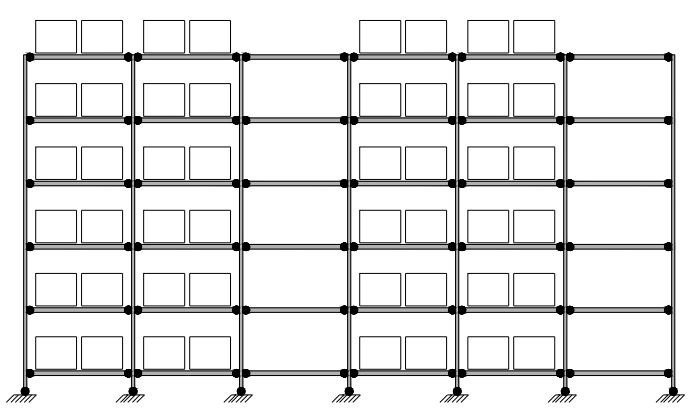

(f) Case 5

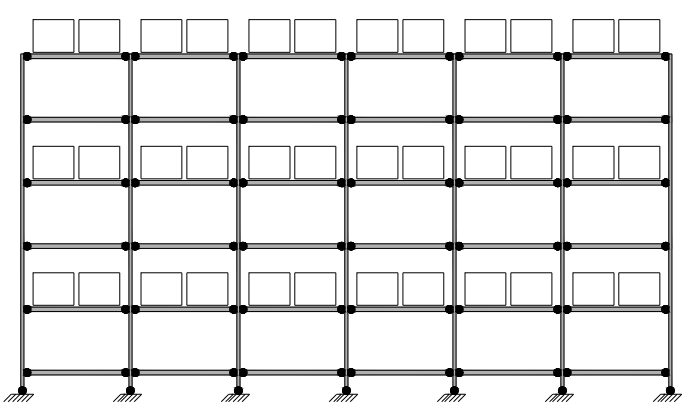

(h) Case 7

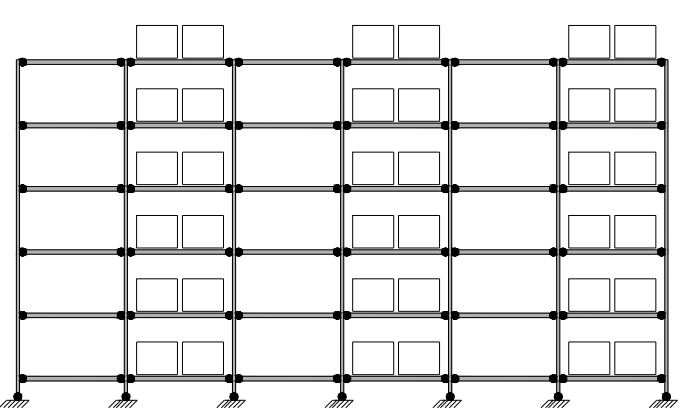

(c) Case 2

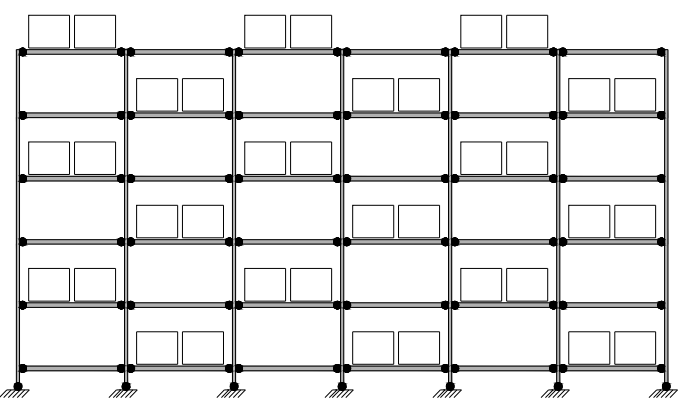

(e) Case 4

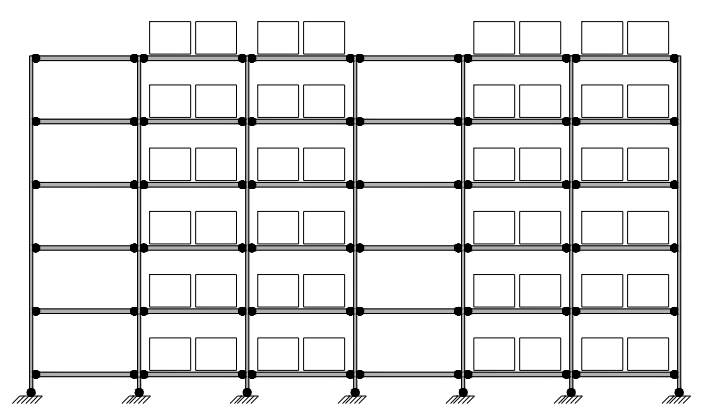

(g) Case 6

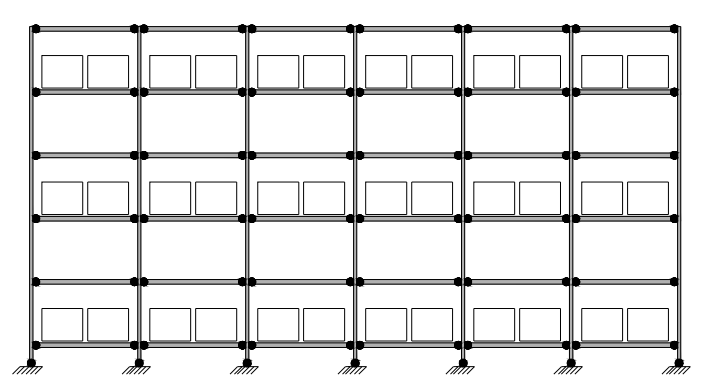

(i) Case 8 


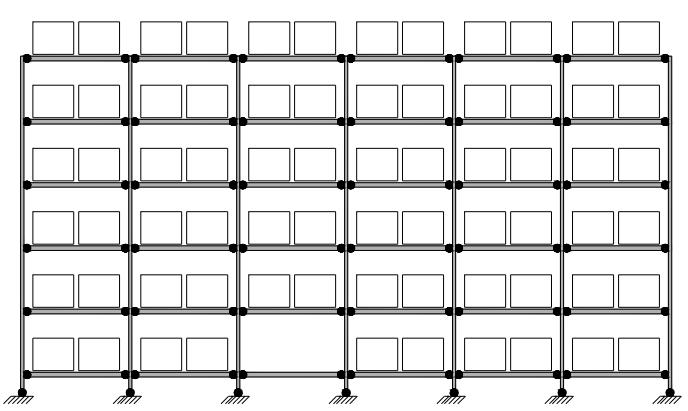

(j) Case 9

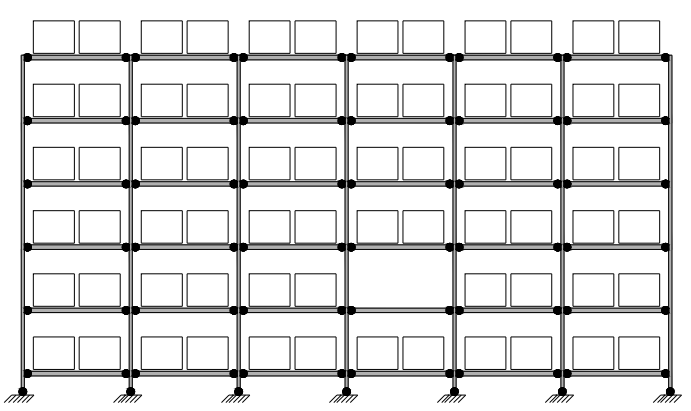

(1) Case 11

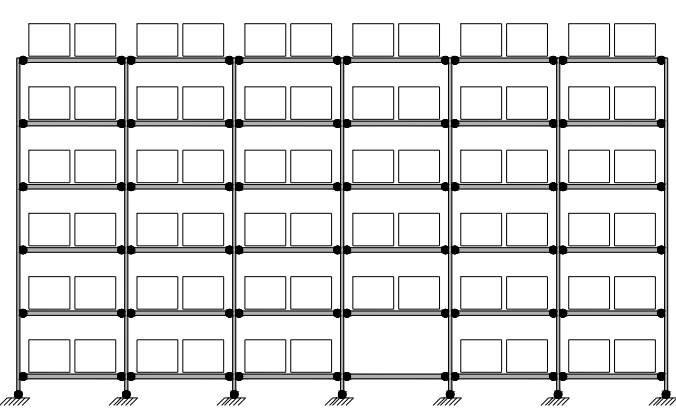

(k) Case 10

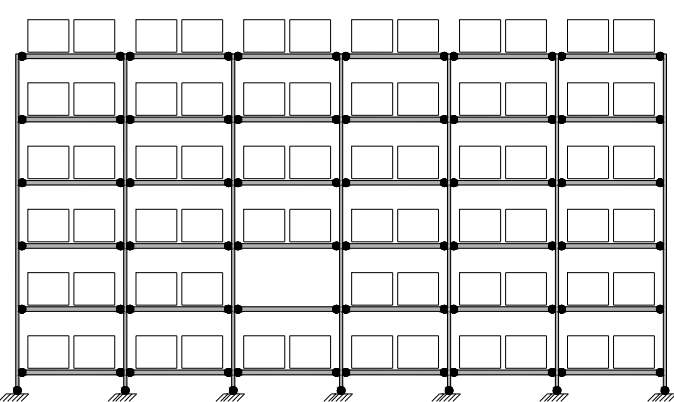

(m) Case 12

Figure 3. Cases of Loading

From the figures it can be seen that without any imperfection that the pairs of load cases are usually mirror images and would give the same results. However, when the geometrical imperfections in the down and cross-aisle directions were added the symmetry was lost and the cases therefore show the effects of the asymmetry.

\subsection{Frame Imperfection}

According to the FEM code [10] the effects of frame imperfections can be considered in the analysis of pallet rack structures by means of an initial out-of-plumb (i.e. initial sway imperfection) or by a closed system of equivalent horizontal forces. In this study the sway imperfections $\phi$ were replaced by a closed system of equivalent horizontal forces. These equivalent horizontal forces were applied at each level and were proportional to the factored vertical loads applied to the structure at the corresponding level as shown in Figure 4.

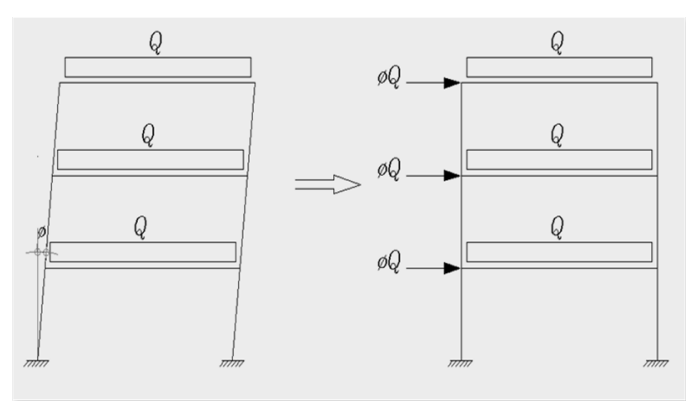

Figure 4. Equivalent Horizontal Forces 
A uniformly distributed load of $1 \mathrm{~N} / \mathrm{m}$ was applied to each beam, and frame imperfections were considered by assigning lateral point loads at each level with a magnitude of $1 \%$ of the applied vertical load.

\subsection{Beam, Upright, Base-plate and Bracing Element and Cross Sections}

The numerical study on the frame was carried out by using a non-linear Finite Element package ABAQUS/CAE [16].

ABAQUS offers a wide range of beam elements and cross sections including "Euler-Brenoulli"-type beams and "Timoshenko" type beams with solid, thin walled closed and thin-walled open sections. The general beam section with Timoshenko-type beam (B31) was selected as the suitable section and element to analyse the beam and upright members.

These elements have two translational degrees and one rotational degree of freedom $\left(\Delta_{x}, \Delta_{y}\right.$ and $\left.\theta_{z}\right)$ at each end and also include the effects of shear flexibility. Each beam member was divided to 24 elements, the bottom upright member was divided into 8 elements and other upright members were divided to 28 elements (the length of each beam element was $100 \mathrm{~mm}$ and the length of each upright element was $50 \mathrm{~mm}$. Typical section values representative of real rack structures were used in the analyses Table 1 show the values used in the analysis.

Table 1. Section Properties

\begin{tabular}{|l|l|l|}
\hline Member & Beams & Uprights \\
\hline Area $\left(\mathrm{mm}^{2}\right)$ & 45.0 & 57.5 \\
\hline Second Moment of Area, down-aisle $\left(\mathrm{mm}^{4}\right)$ & $0.78 * 10^{6}$ & $0.60 * 10^{6}$ \\
\hline $\begin{array}{l}\text { Second Moment of Area, cross-aisle } \\
\left(\mathrm{mm}^{4}\right)\end{array}$ & $0.32 * 10^{6}$ & $0.20 * 10^{6}$ \\
\hline Torsion Constant $\left(\mathrm{mm}^{4}\right)$ & $1.10 * 10^{6}$ & $0.80 * 10^{6}$ \\
\hline Young's Elastic Modulus, N/mm & $210 * 10^{9}$ & $210^{2} 10^{9}$ \\
\hline
\end{tabular}

The members forming the horizontal and diagonal bracing were analysed using truss elements type T3D2 (2-node linear displacement) with cross sectional area $90 \mathrm{~mm}^{2}$, these elements are slender structural members that can transmit only axial force and do not transmit moments. Finally, the base-plate was analysed using shell element type S4R (4-node doubly curved thin shell). The area of this base-plate was taken be $(150 * 150 \mathrm{~mm})$ with a total thickness of $3 \mathrm{~mm}$ and each base-plate was divided into 64-elements. In addition these elements have three translational and three rotational degrees of freedom at each node.

\subsection{Convergence Study}

This convergence study was carried out with the aim of determining the required number of elements that were needed for the finite element model for the beam and shell elements. Moreover for each element type different numbers of element were used and the effect of on the displacements of the models upper node investigated. 


\subsubsection{Beam element}

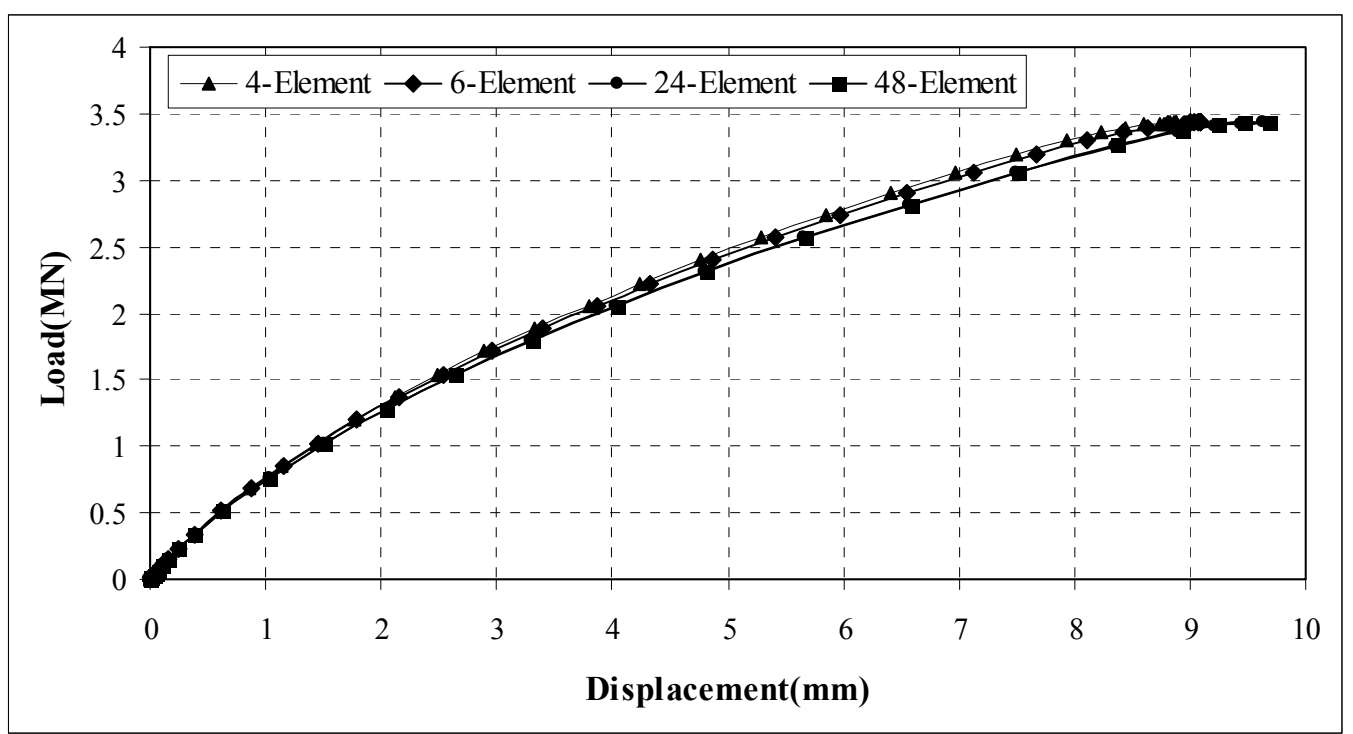

Figure 5. Load-Displacement Curves for Different Number of Elements

Figure 5 shows a plot of the different number of elements $(4,6,24$ and 28-elements) used for the beams. The load-deflection curves are plotted for each number of elements for the general case of a fixed-base with no imperfection.

As the reader can see this figure showed good agreement between the use of 24-elements and 48 -elements for the same frame with a maximum percentage error of $0.01 \%$. Hence the number of elements used in the analysis for all cases was 24 .

\subsubsection{Shell element}

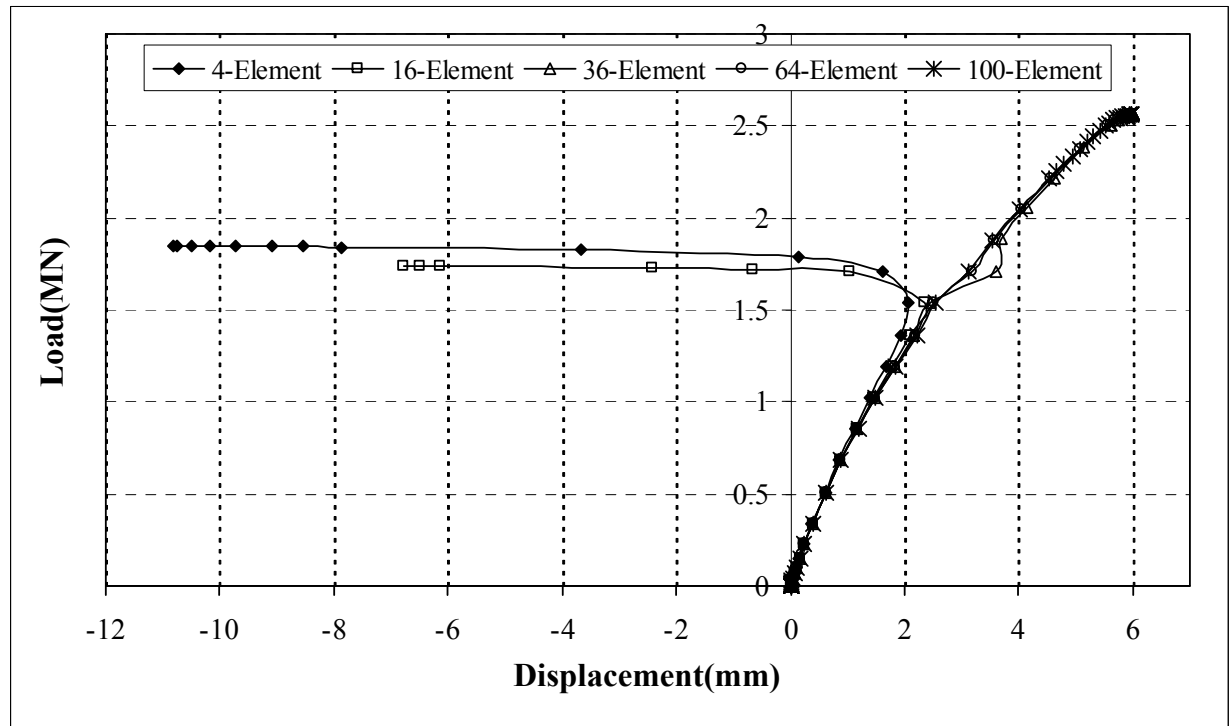

Figure 6. Load-Displacement Curves for Different Numbers of Elements

Figure 6 shows the different number of elements (4, 16, 36, 64 and 100-elements) that were used for shells and the load-deflection curves are plotted for each discretisation for the general case semi-rigid base-plate with no imperfection. 
This figure shows that when 4 and 16-shell elements were used the frame swayed in the negative down-aisle direction and when 36, 64 and 100-shell elements were used the frame swayed in the opposite direction. As no imperfections in either load or geometry occurred in the test model the direction of sway was arbitrary. In addition the use of 36, 64 and 100-shell elements gave a good agreement between them with a maximum percentage error of $0.007 \%$. So this means that finer meshes help to obtain accurate results. It was found that discretising the base-plate to 64-elements gave sufficient, accurate results.

\subsection{Semi-rigid Connections}

Semi-rigid connections were used to connect uprights and beams, and uprights and base-plates. Modelling the semi-rigid connection between the uprights and beams and uprights and base-plates required the use of the spring joint element that represents the required stiffnesses, the ABAQUS SPRING2 element was used. The joint element used to model the beam-upright connection had three translational and three rotational degrees of freedom at each end. The translational stiffnesses of the connection were taken to be infinite. The rotation stiffness about an axis along a cross-aisle direction $\left(z\right.$-axis) was taken to be $0.15 * 10^{6} \mathrm{kNmm} / \mathrm{rad}$. The rotation stiffness about a vertical axis $(y$-axis) was assumed to be zero, and about an axis lying along the beam $(x$-axis $)$ was taken to be infinite.

The joint element used to model the upright-base plate connection such upright-beam connection had three translational and three rotational degrees of freedom at each end. The translational stiffnesses of the connection were taken to be infinite. The rotation stiffness about axis along a cross-aisle direction $(z-$ axis $)$ was taken to be $0.15 * 10^{6} \mathrm{kNmm} / \mathrm{rad}$. The rotation stiffness about a vertical axis $(y$-axis) was taken to be infinite, and about an axis lying along the beam $\left(x\right.$-axis) was taken to be $0.30 * 10^{6} \mathrm{kNmm} / \mathrm{rad}$.

\section{ANALYSIS AND DISCUSSION}

For each load case a static analysis was conducted followed by non-linear geometric analysis to obtain the maximum load.

\subsection{Without Initial Imperfection Cases}

Figures 7(a), 7(b) and 7(c) show the effects of different base conditions on the displacements of the model's upper left node under different combinations. From Figures 7(a) and 7(c) frames with fixed and pinned bases reached their maximum capacity when they were partially loaded under load case 8. The frame with a semi-rigid base-plate reached its maximum capacity when it was fully loaded (general case) and with no imperfection. The maximum reduction from the fully loaded case was $47 \%$. The effects of small imperfections on the maximum capacity can be clearly seen. The full details of maximum loads in each load case compared against the fully loaded case are given in tables 2-4. The percentage increases/reductions in capacity are compared against the fully loaded case for each imperfection condition. 


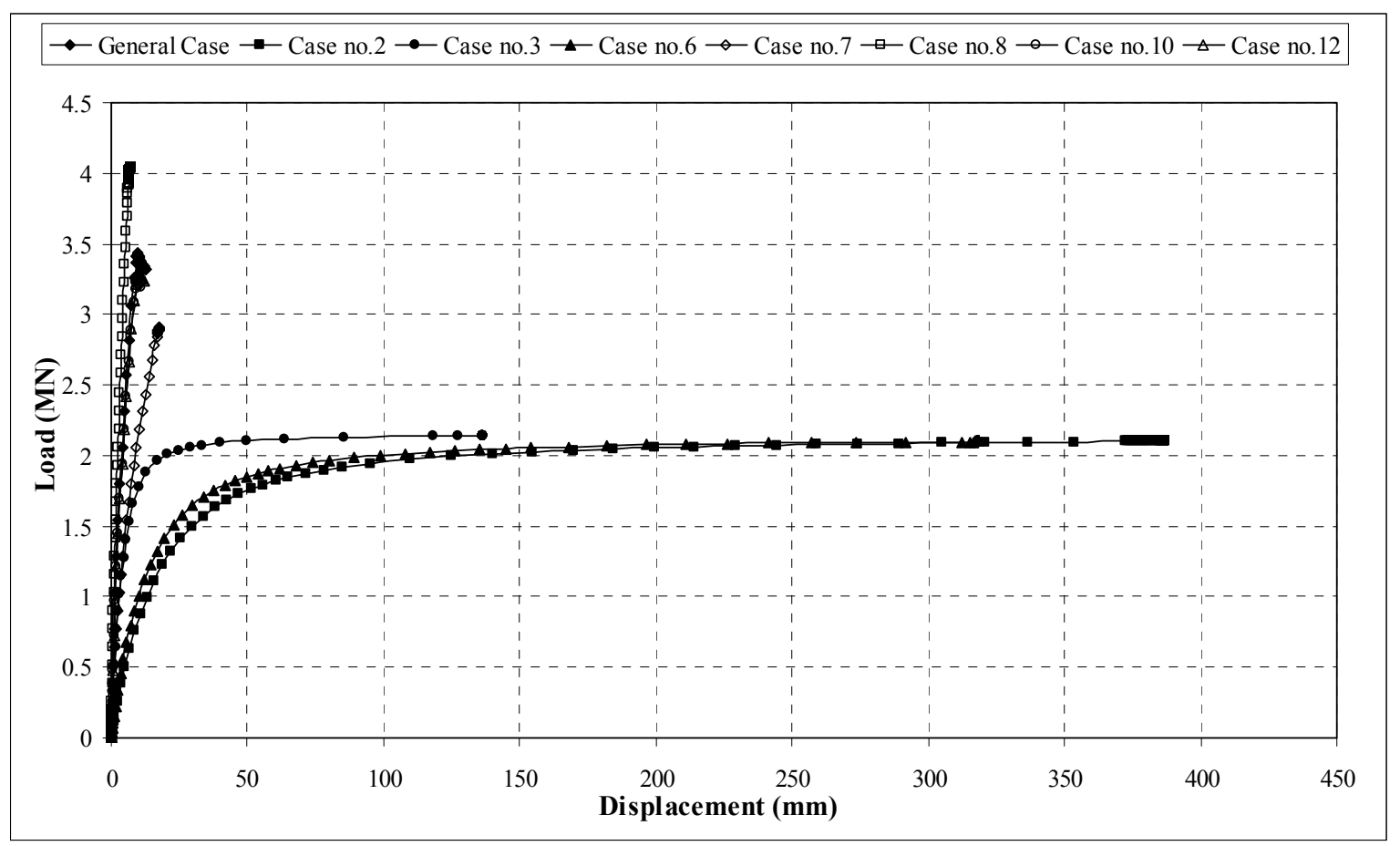

(a) Rigid Base

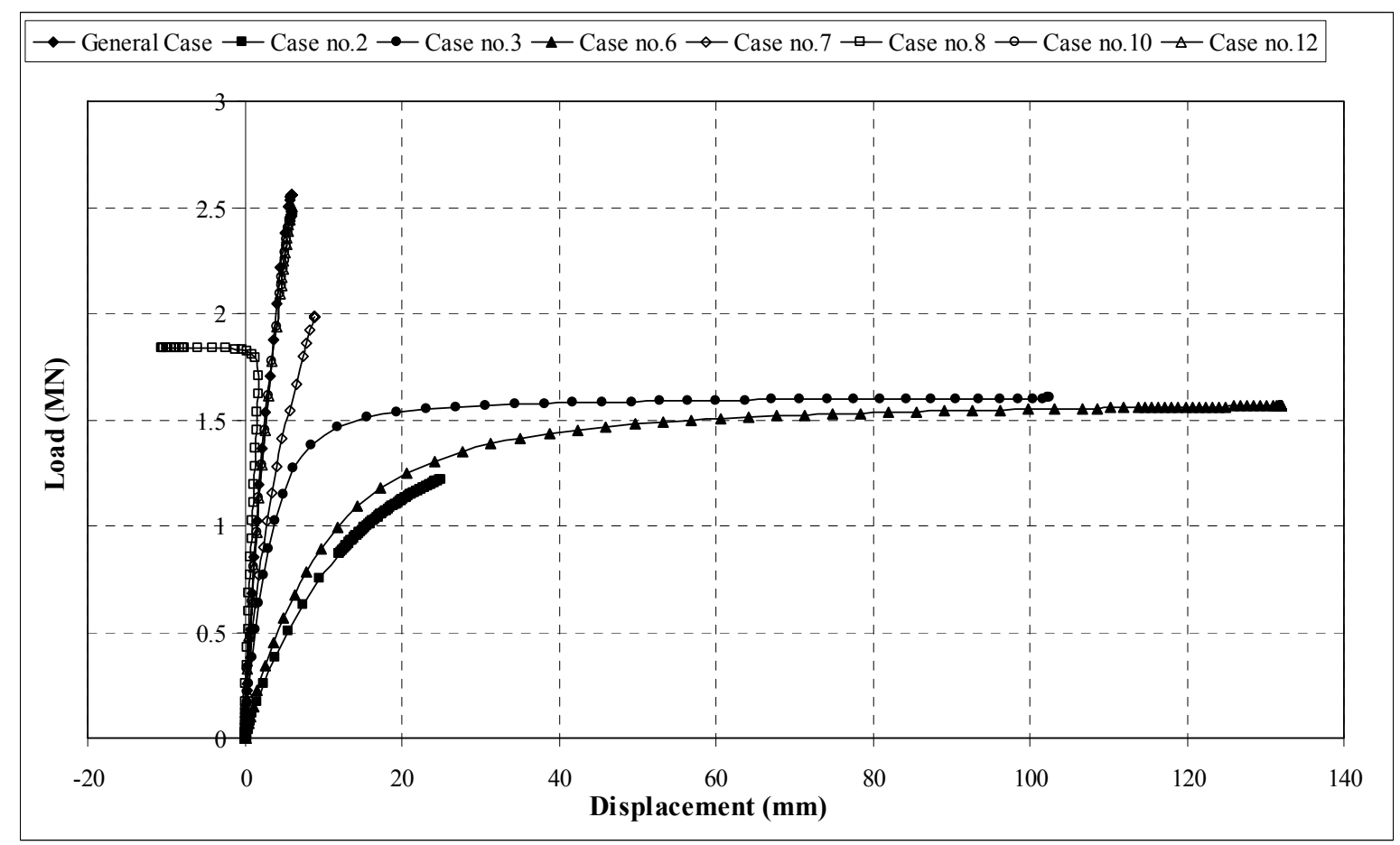

(b) Semi-rigid Base 


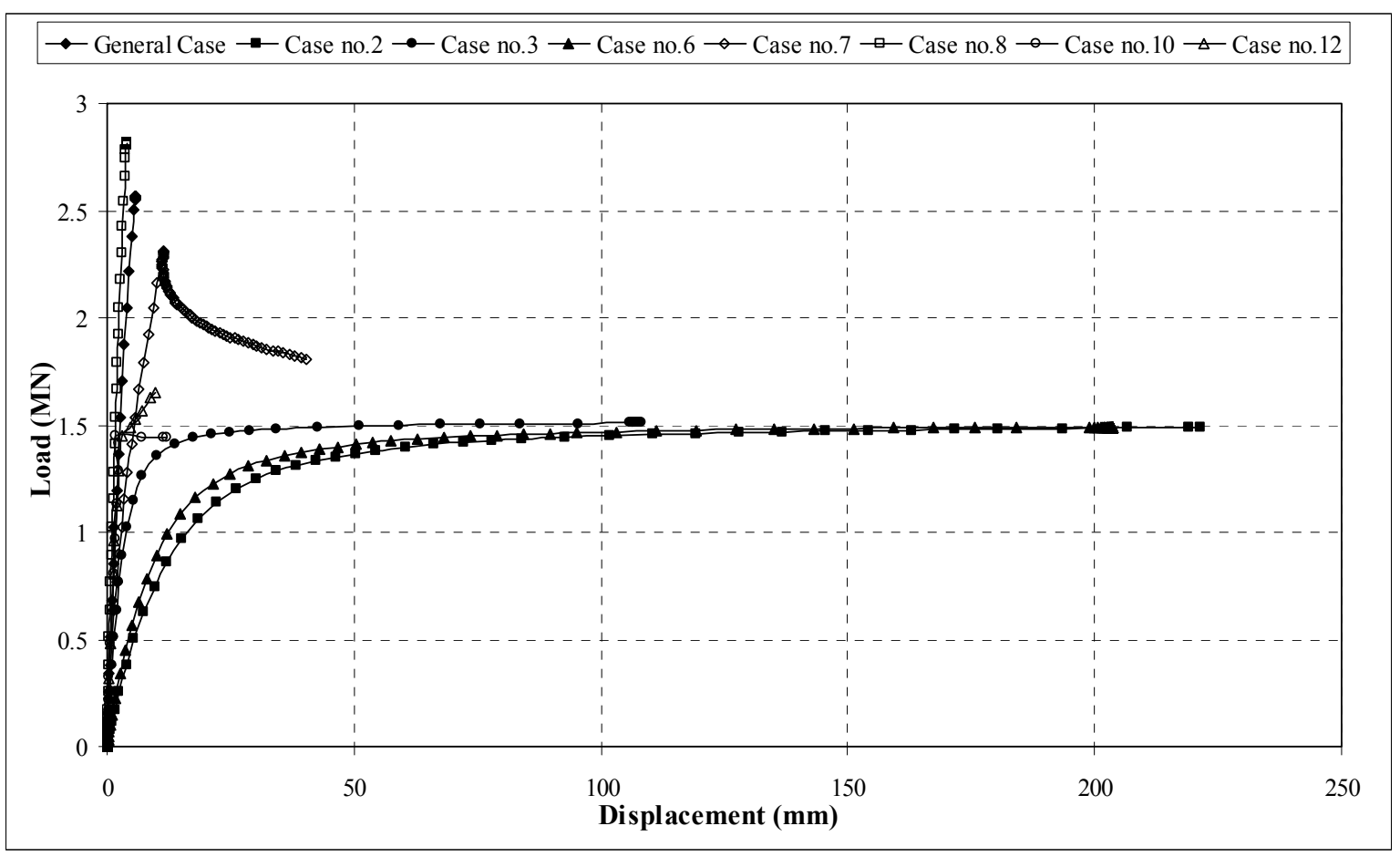

(c) Pinned Base

Figure 7. Load-Displacement Curves for All Cases

Table 2. Influence of Cases of Loading on the Rigid-base Frame

\begin{tabular}{|c|c|c|c|c|c|c|}
\hline \multirow{2}{*}{ Load case } & \multicolumn{6}{|c|}{ Maximum load-capacity of the frame $(\mathrm{kN})$} \\
\hline & \multicolumn{2}{|c|}{ without out of plumb } & \multicolumn{2}{|c|}{ With $1 \% x$-direction } & \multicolumn{2}{|c|}{ With $1 \%(x+z)$-directior } \\
\hline General case & 3432.1 & & 2105.9 & & 2082.4 & \\
\hline Case No.1 & 2099.1 & $-38.8 \%$ & 2123.9 & $0.9 \%$ & 2109.8 & $1.3 \%$ \\
\hline Case No.2 & 2099.2 & $-38.83 \%$ & 2064.0 & $-2.0 \%$ & 2051.0 & $-1.5 \%$ \\
\hline Case No.3 & 2136.9 & $-37.7 \%$ & 2091.0 & $-0.7 \%$ & 2074.6 & $-0.4 \%$ \\
\hline Case No.4 & 2137.2 & $-37.7 \%$ & 2099.2 & $-0.3 \%$ & 2083.0 & $0.0 \%$ \\
\hline Case No.5 & 2097.7 & $-38.9 \%$ & 2103.9 & $-0.1 \%$ & 2089.0 & $0.3 \%$ \\
\hline Case No.6 & 2098.1 & $-38.9 \%$ & 2060.8 & $-2.1 \%$ & 2042.8 & $-1.9 \%$ \\
\hline Case No.7 & 2904.6 & $-15.4 \%$ & 1830.0 & $-13.1 \%$ & 1822.8 & $-12.5 \%$ \\
\hline Case No.8 & 4047.3 & $17.9 \%$ & 2415.9 & $14.7 \%$ & 2401.7 & $15.3 \%$ \\
\hline Case No.9 & 3244.2 & $-5.5 \%$ & 1982.6 & $-5.9 \%$ & 1967.0 & $-5.5 \%$ \\
\hline Case No.10 & 3244.3 & $-5.5 \%$ & 1982.6 & $-5.9 \%$ & 1967.0 & $-5.5 \%$ \\
\hline Case No.11 & 3290.5 & $-4.1 \%$ & 2000.9 & $-5.0 \%$ & 1985.3 & $-4.7 \%$ \\
\hline Case No.12 & 3290.9 & $-4.1 \%$ & 2000.7 & $-5.0 \%$ & 1985.1 & $-4.7 \%$ \\
\hline
\end{tabular}


Table 3. Influence of Cases of Loading on the Semi-rigid-base Frame

\begin{tabular}{||l|ll|ll|ll||}
\hline \multirow{2}{*}{ Load case } & \multicolumn{5}{|l|}{ Maximum load-capacity of the frame (N) } \\
\cline { 2 - 8 } & \multicolumn{2}{|l|}{ without out of plumb } & \multicolumn{2}{l||}{ With 1\% -direction } & \multicolumn{2}{l||}{ With 1\% $(x+z)$-direction } \\
\hline \hline General case & 2560.2 & & 1545.7 & & 1524.1 & \\
\hline Case No.1 & 1570.5 & $-38.7 \%$ & 1475.9 & $-4.5 \%$ & 1480.4 & $-2.9 \%$ \\
\hline Case No.2 & 1568.8 & $-38.73 \%$ & 1467.5 & $-5.1 \%$ & 1477.6 & $-3.0 \%$ \\
\hline Case No.3 & 1601.1 & $-37.5 \%$ & 1490.9 & $-3.5 \%$ & 1463.3 & $-4.0 \%$ \\
\hline Case No.4 & 1600.8 & $-37.5 \%$ & 1499.6 & $-3.0 \%$ & 1475.4 & $-3.2 \%$ \\
\hline Case No.5 & 1578.8 & $-38.3 \%$ & 1510.8 & $-2.3 \%$ & 1477.3 & $-3.1 \%$ \\
\hline Case No.6 & 1564.4 & $-38.9 \%$ & 1502.4 & $-2.8 \%$ & 1472.7 & $-3.4 \%$ \\
\hline Case No.7 & 1988.3 & $-22.3 \%$ & 1348.9 & $-12.7 \%$ & 1306.9 & $-14.3 \%$ \\
\hline Case No.8 & 1840.1 & $-28.1 \%$ & 1701.0 & $10.0 \%$ & 1650.7 & $8.3 \%$ \\
\hline Case No.9 & 2459.6 & $-3.9 \%$ & 1462.9 & $-5.4 \%$ & 1441.2 & $-5.4 \%$ \\
\hline Case No.10 & 2458.6 & $-4.0 \%$ & 1461.0 & $-5.5 \%$ & 1438.0 & $-5.7 \%$ \\
\hline Case No.11 & 2504.5 & $-2.2 \%$ & 1488.2 & $-3.7 \%$ & 1466.8 & $-3.8 \%$ \\
\hline Case No.12 & 2504.3 & $-2.2 \%$ & 1488.1 & $-3.7 \%$ & 1466.8 & $-3.8 \%$ \\
\hline \hline
\end{tabular}

Table 4. Influence of Cases of Loading on the Pinned-base Frame

\begin{tabular}{|c|c|c|c|c|c|c|}
\hline \multirow{2}{*}{ Load case } & \multicolumn{6}{|c|}{ Maximum load-capacity of the frame $(\mathrm{kN})$} \\
\hline & \multicolumn{2}{|c|}{ without out of plumb } & \multicolumn{2}{|c|}{ With $1 \% x$-direction } & \multicolumn{2}{|c|}{ With $1 \%(x+z)$-directior } \\
\hline General case & 2563.0 & & 1470.4 & & 1454.1 & \\
\hline Case No.1 & 1487.9 & $-41.9 \%$ & 1482.3 & $0.8 \%$ & 1466.6 & $0.9 \%$ \\
\hline Case No.2 & 1488.1 & $-41.94 \%$ & 1455.1 & $-1.0 \%$ & 1438.2 & $-1.1 \%$ \\
\hline Case No.3 & 1509.3 & $-41.1 \%$ & 1467.6 & $-0.2 \%$ & 1451.3 & $-0.2 \%$ \\
\hline Case No.4 & 1550.6 & $-39.5 \%$ & 1473.1 & $0.2 \%$ & 1457.1 & $0.2 \%$ \\
\hline Case No.5 & 1534.0 & $-40.1 \%$ & 1476.6 & $0.4 \%$ & 1459.9 & $0.4 \%$ \\
\hline Case No.6 & 1491.3 & $-41.8 \%$ & 1455.4 & $-1.0 \%$ & 1438.5 & $-1.1 \%$ \\
\hline Case No.7 & 2311.3 & $-9.8 \%$ & 1289.8 & $-12.3 \%$ & 1285.0 & $-11.6 \%$ \\
\hline Case No.8 & 2813.1 & $9.8 \%$ & 1685.4 & $14.6 \%$ & 1668.6 & $14.8 \%$ \\
\hline Case No.9 & 1450.4 & $-43.4 \%$ & 1395.0 & $-5.1 \%$ & 1379.5 & $-5.1 \%$ \\
\hline Case No.10 & 1450.8 & $-43.4 \%$ & 1395.0 & $-5.1 \%$ & 1379.5 & $-5.1 \%$ \\
\hline Case No.11 & 1448.9 & $-43.5 \%$ & 1419.7 & $-3.4 \%$ & 1403.5 & $-3.5 \%$ \\
\hline Case No.12 & 1465.0 & $-42.8 \%$ & 1419.4 & $-3.5 \%$ & 1403.4 & $-3.5 \%$ \\
\hline
\end{tabular}


Figure 8 shows the total reaction force in the columns calculated by the summation of reaction force in all columns; these values show good agreement with the total load capacity for each cases of loading. In addition Figure 6 shows the collapse mode for the critical case (case 2).

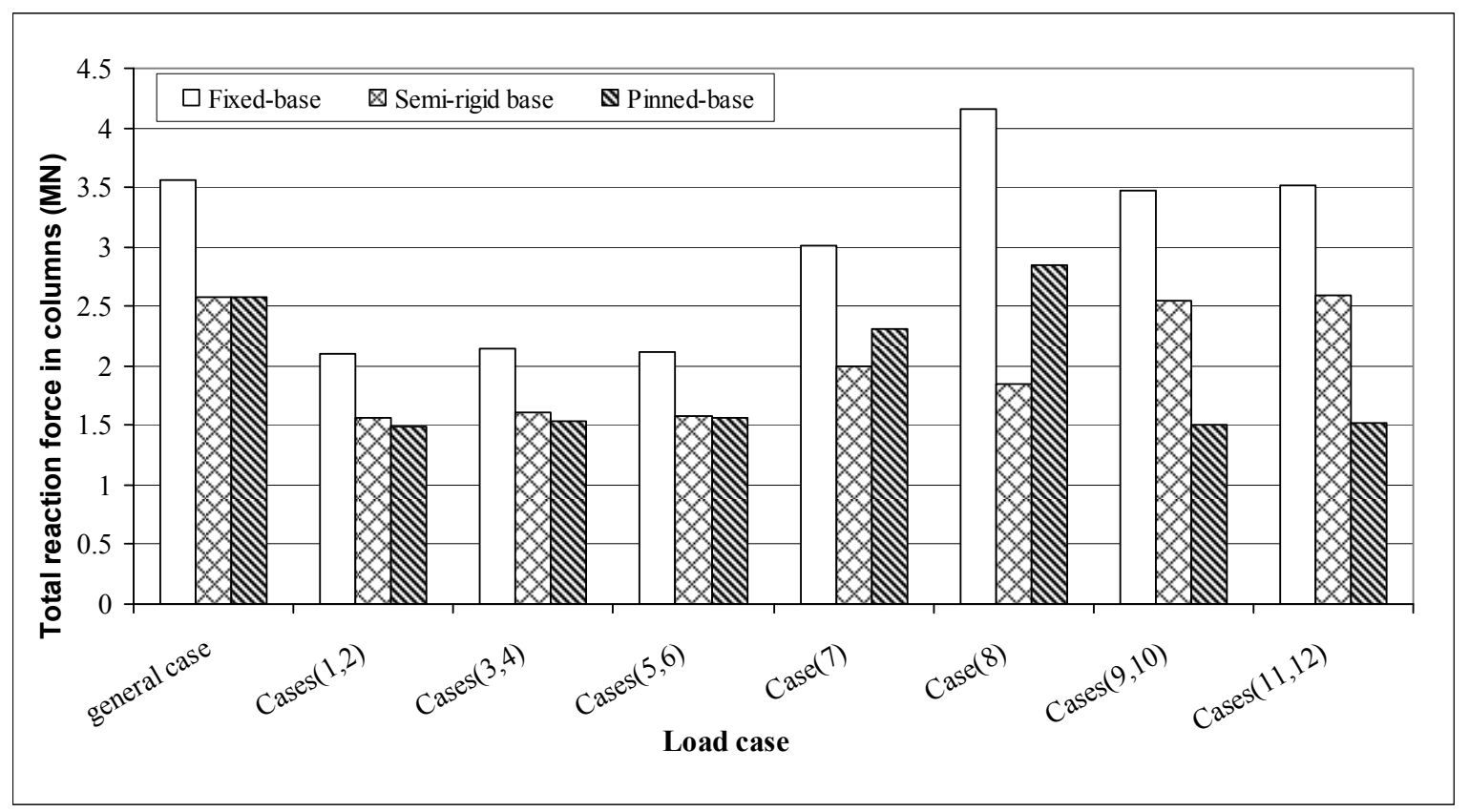

Figure 8. Total Reaction Force in Columns for All Cases with No Imperfection

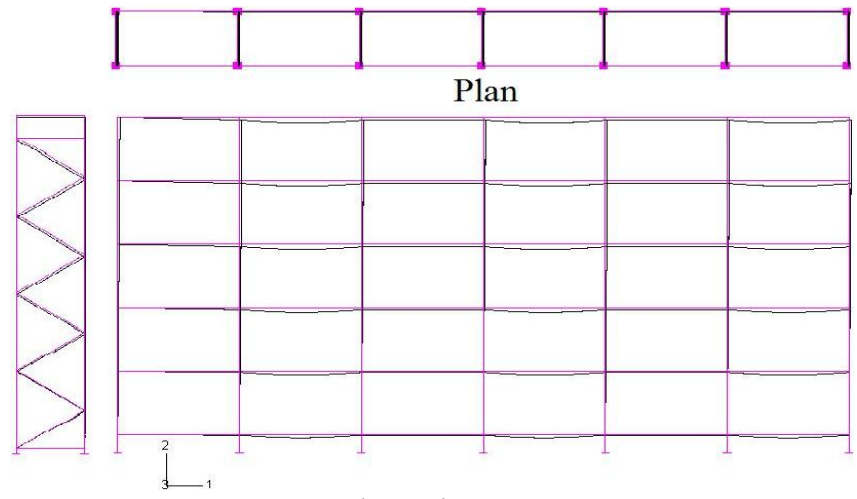

Front elevation

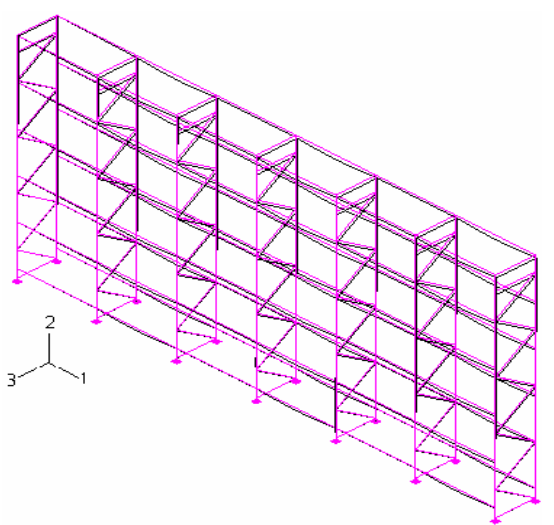

Isometric view

Figure 9. Collapse Mode for Case 2 (Semi-rigid Base-plate Frame)

\subsection{Imperfection in Down and Cross-aisle Directions}

Figures 10(a), 10(b) and 10(c) show the reductions in capacities of the frames when subjected to geometrical imperfections for the same node as used in Figure 7. These figures show 7-different combinations of loading in addition to the general fully loaded case for (rigid, semi-rigid and pinned bases respectively).

As can be seen in figures 10(a), 10(b) and 10(c) and Tables 2-4, the frames reached their maximum capacity when they were partially loaded, normally case 8 . The minimum capacity, below that of the loaded frame was usually either case 11 or case 12 which has one element in the lowest bay unloaded in conformance with the common loadings used in design (see Beale and Godley [10, 11]. 


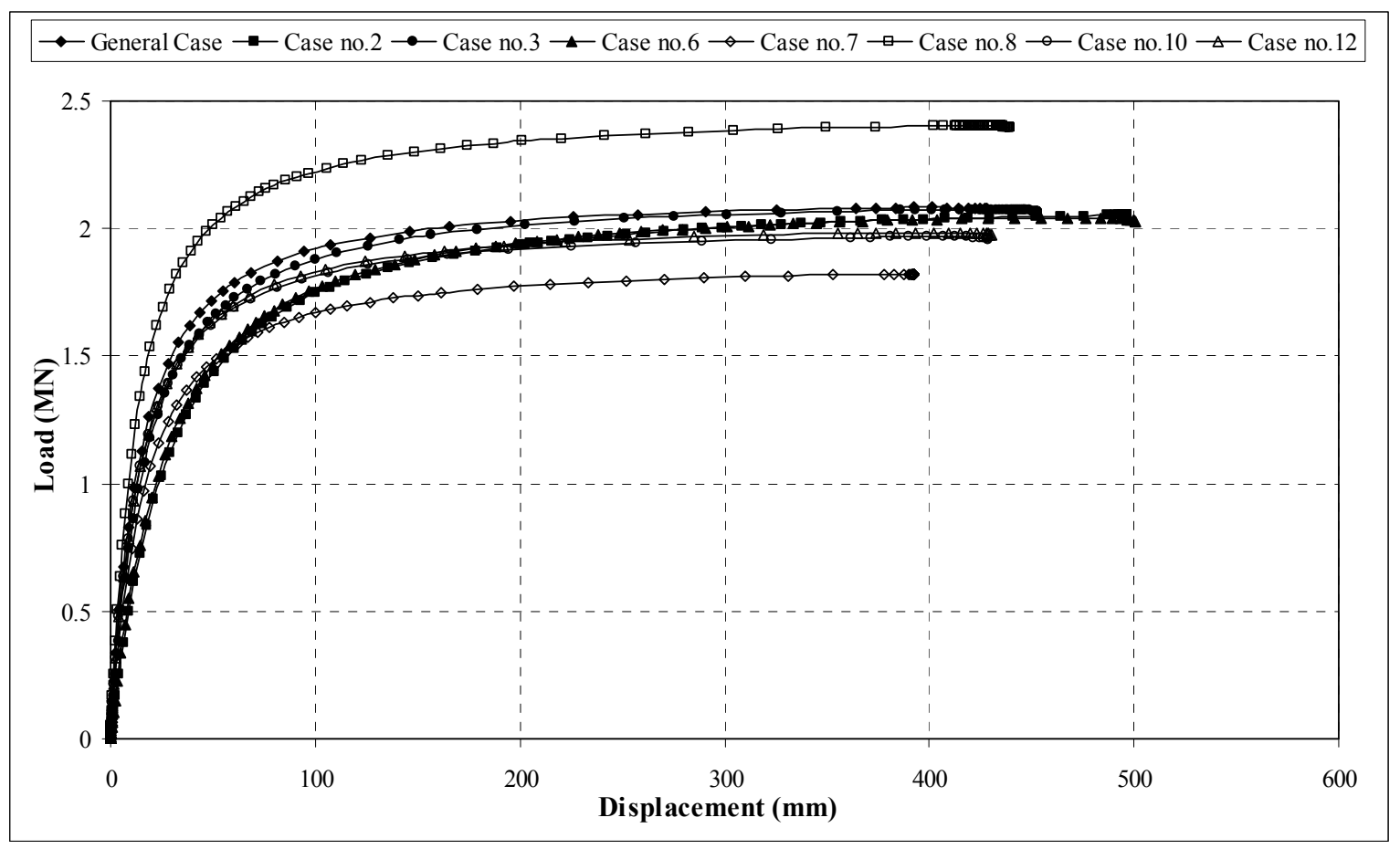

(a) Rigid Base

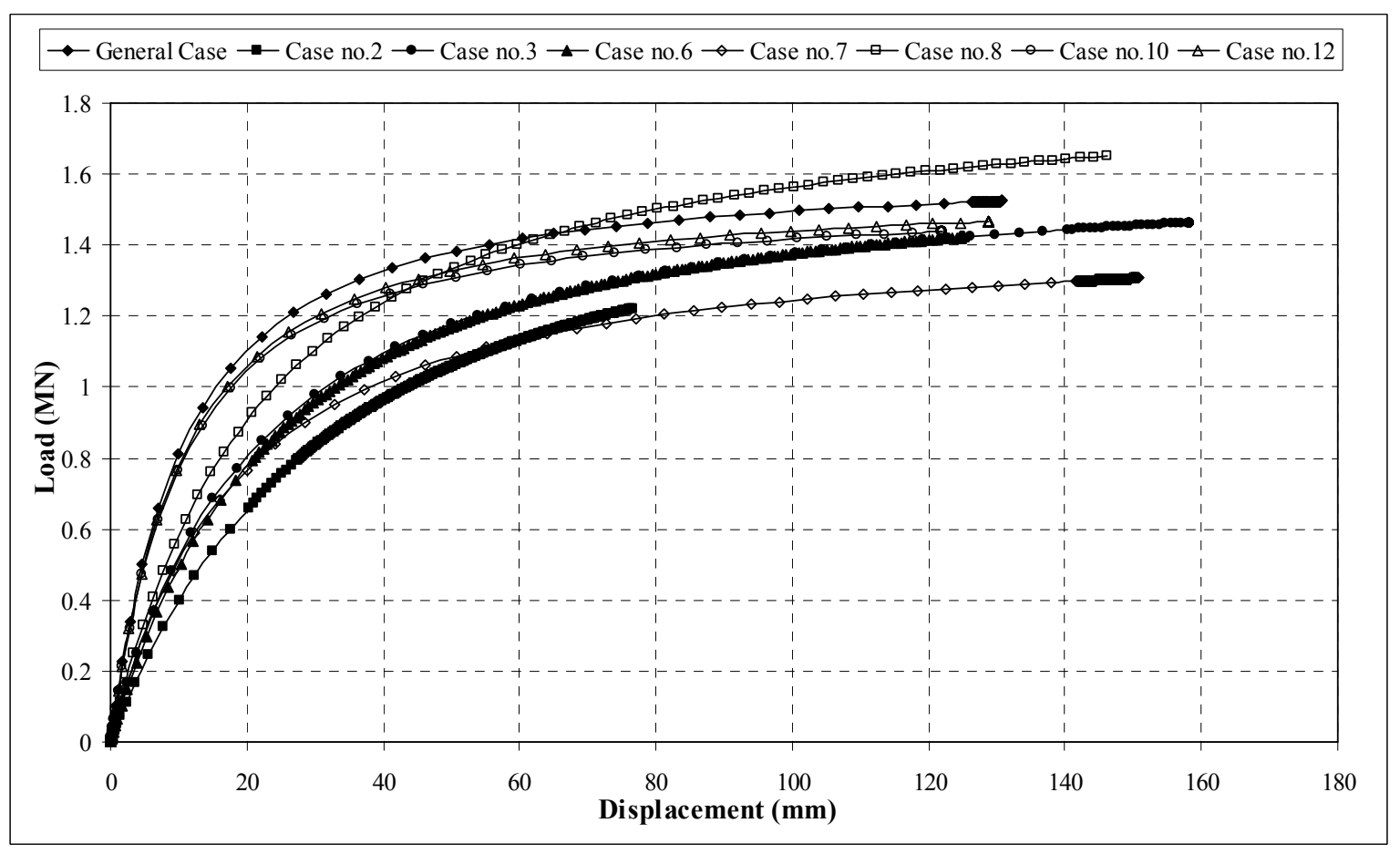

(b) Semi-rigid Base-plate 


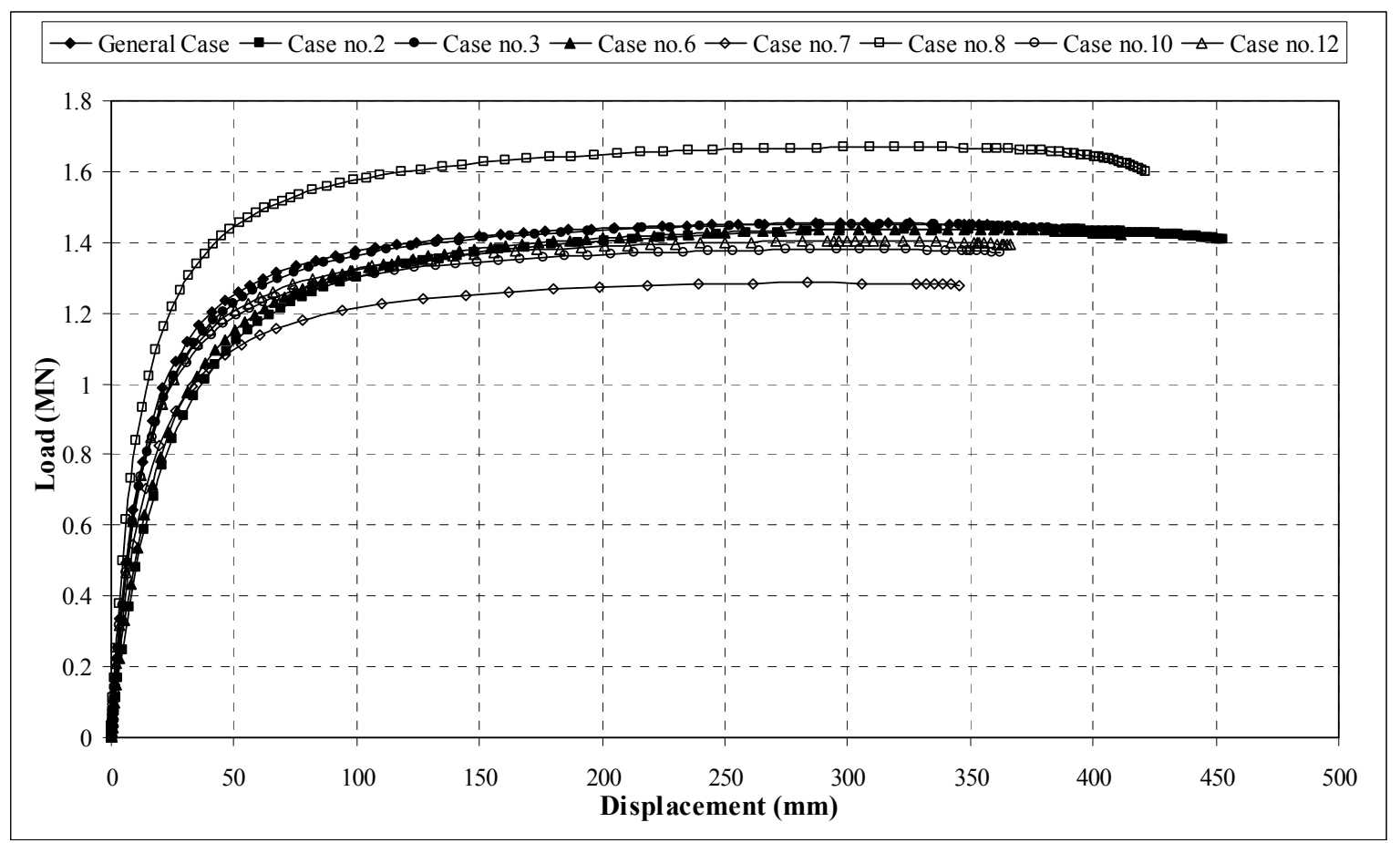

(c) Pinned Base

Figure 10. Load-Displacement Curves for All Cases with Side Imperfection

Figures 11 shows the total reaction force in the columns calculated by the summation of reaction force in all columns; these values have shown good agreement with total load capacity for each cases of loading. In addition Figure 8 shows the collapse mode for the case 7 .

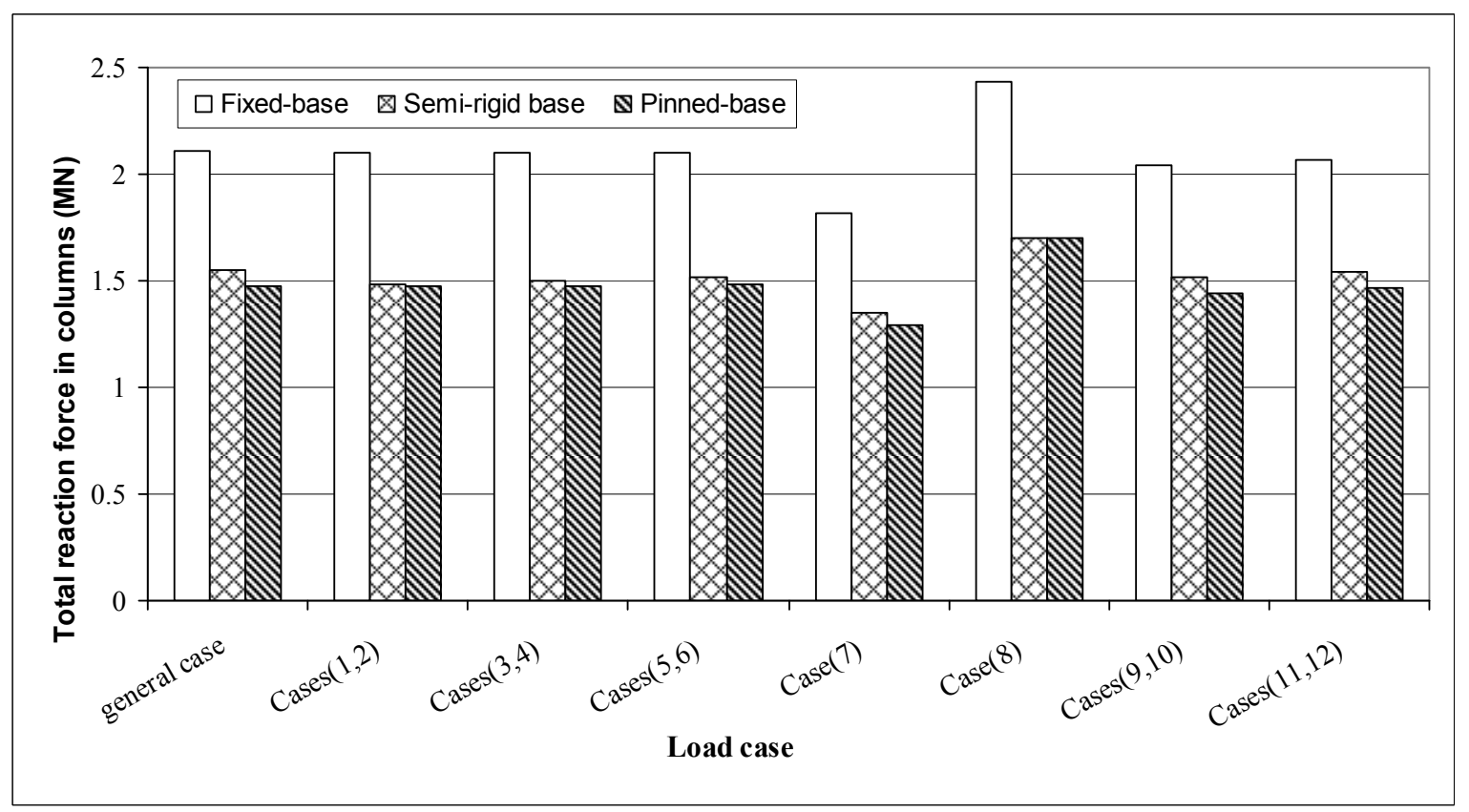

Figure 11. Total Reaction in Columns for All Cases with $1 \%(x+z)$-direction 


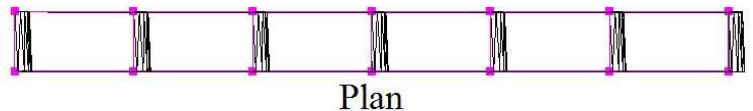

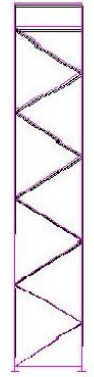

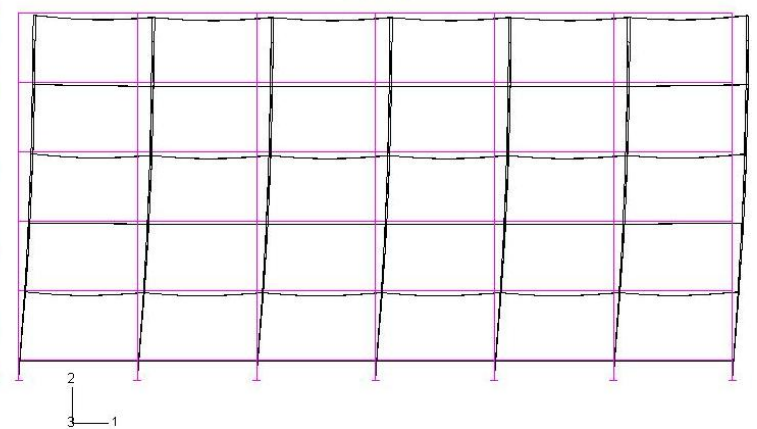

Front elevation

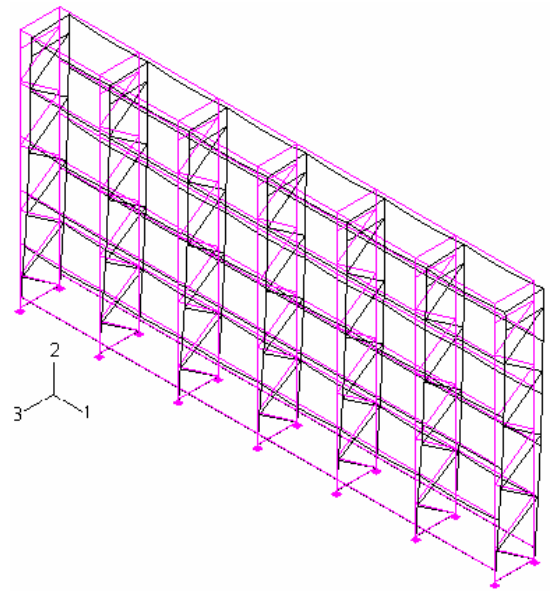

Isometric view

Figure 12. Collapse Mode for Case 7 (Semi-rigid Base-plate Frame)

In all cases analysed the effects of imperfections in reducing the maximum load carrying capacity of frames are clearly seen. Whether the imperfections are caused by geometric imperfection or load asymmetry the frames capacity is reduced by up to $50 \%$ of the fully loaded perfect frame. In addition, the load deflection plots show that the failure in the perfect frame is by structural instability with a steep descending curve.

\section{CONCLUSIONS}

In the present study, the non-linear static responses of pallet racks with fixed-bases, semi-rigid bases or pinned-bases with and without initial imperfections have been investigated under different cases of loading. The study found that the frame's behaviour is affected by partial loads and by lateral loads representing initial imperfections. When a comparison was carried out between all cases with no imperfection it was found that the most critical type loading occurred when the loads were removed on one bay for all lifts but when imperfections in down and cross-aisle directions are present the most critical loading occurs when the loads are removed from one level. In all cases of loading the pallet rack structure failed in a global sway mode except the fully loaded case without imperfections.

\section{REFERENCES}

[1] Markazi, F.D., Godley, M.H.R. and Beale, R.G., "Experimental Analysis of Semi-Rigid Boltless connections", Thin Walled Structures, 1997, Vol. 27, No. 1, pp. 53-87.

[2] Beale, R.G. and Godley, M.H.R. "Problems Arising with Pallet Rack Semi-rigid Base-plates", Proceedings of the First International Conference on Steel and Composite Structures, Pusan, pp. 699-706.

[3] Godley, M.H.R. "The Behaviour of Storage Racking Baseplates", Proceedings of the Sixth International Conference on Steel and Aluminium Structures (ICSAS'07), 2007, Oxford, pp.433-440.

[4] Baldassino, N. and Bernuzzi, C., "Analysis and Behaviour of Steel Storage Pallet Racks", Thin-Walled Structures, 2000, Vol. 37, pp. 277-304.

[5] Abdel-Jaber, M.S., "The Influence of Semi-Rigid Connections on the Behaviour of Beam and Column Structural System”, PhD Thesis: Oxford Brookes University, UK, 2002. 
[6] Sarawit, A.T. and Pekoz, T., "Design of Industrial Storage Racks", Proceedings sixteenth International Specialty Conference on Cold-Formed Steel Structures", Orlando, 2002, pp.369-384.

[7] Sarawit, A.T., "Cold-Formed Steel Frame and Beam-Column Design”, PhD Thesis: Cornell University, US, 2003.

[8] Freitas, A.M.S., Freitas, M.S.R. and Souza, F.T., "Analysis of Steel Storage Rack Columns", Journal of Constructional Steel Research, 2005, Vol. 61, No. 8, pp. 1135-1146.

[9] Beale, R.G., "The Influence of Semi-Rigid Connections on the Non-Linear Behaviour of Pallet Rack and Scaffold Structures". PhD Thesis: Oxford Brookes University, UK, 2004.

[10] Federation Europeéne de la Manutention, Section X, "Recommendations for the Design of Steel Pallet Racking and Shelving", 2000.

[11] The Rack Manufacturers' Institute, "Specification for the Design, Testing and Utilization of Industrial Steel Storage Racks", 1997.

[12] Beale, R.G. and Godley, M.H.R , "The Design of the Pallet Program", Proceedings of the sixteenth Specialty Conference on Cold-Formed Steel Structures, Orlando, 2002, pp.353-368.

[13] Beale, R.G. and Godley, M.H.R., "Developments in the Pallet Program", Advances in Structures, Sydney, 2003, pp. 237-243.

[14] Morz, K., Olsson, A. and Sandberg, G., "Parameter Studies on Pallet Racks", SP Swedish National Testing and Research Institute, SP Report 1998-2, 1998.

[15] Olsson, A. and Sandberg, G., "Reduction of Load Carrying Capacity in Pallet Racks due to Damaged Upright Beams", Tenth Nordic Seminar on Computational Mechanics, Tallinn, 1997, pp. 278-281.

[16] ABAQUS ANALYSIS User's Manual, Version 6.4, Hibbitt, Karlsson \& Sorensen, Inc., 2003. 


\title{
EFFECTS OF STRAIN REGIMES ON THE BEHAVIOUR OF HEADED STUD SHEAR CONNECTORS FOR COMPOSITE STEEL-CONCRETE BEAMS
}

\author{
O. Mirza ${ }^{1}$ and B. $\mathrm{Uy}^{2, *}$ \\ ${ }^{1}$ PhD Candidate, School of Engineering, University of Western Sydney \\ ${ }^{2}$ Professor and Head of School of Engineering, University of Western Sydney \\ Locked Bag 1797 Penrith South DC, NSW 1797, Australia \\ *(Corresponding author: E-mail: b.uy@uws.edu.au)
}

Received: 5 August 2008; Revised: 14 October 2008; Accepted: 20 November 2008

\begin{abstract}
In composite steel-concrete beam construction, one of the most common methods to evaluate shear connector strength and behaviour is through a push test. Push tests have been used as early as the 1960's to predict the strength and behaviour of shear studs in solid slabs. The performance of steel-concrete composite structures is greatly dependent on the load-slip characteristics of shear connectors. Significant research work has been performed on composite beams with regard to their stiffness and ductility of the shear connectors for both solid and profiled slabs. This paper describes the strength and ductility of shear connectors in composite beams with both solid and profiled steel sheeting slabs when different strain regimes are imposed on the concrete element. An accurate non-linear finite element model using ABAQUS is developed herein to study the behaviour of shear connectors for both solid and profiled steel sheeting slabs. The reason for employing different strain regimes in composite steel-concrete beams is to properly simulate the behaviour of shear connectors in composite beams where trapezoidal slabs are used. The pertinent results obtained from the finite element analysis were verified against independent experimental results and existing design standards. Based on the finite element analysis and the experimental results, it is evident that the strength and the load-slip behaviour of composite steel-concrete beams are greatly influenced by the strain regimes existent in the concrete element.
\end{abstract}

Keywords: Composite steel-concrete beams; strain regimes; finite element method; shear connectors

\section{INTRODUCTION}

The push test is meant to determine the basic strength of headed stud shear connectors in composite steel-concrete beams. It is always considered reliable as a lower bound method for the prediction of strength and stiffness in real composite steel-concrete beams. The stress state in and around the headed shear studs are highly complex. Metal decking used to form composite slabs has also used standard push tests to assess strength and ductility of headed stud shear connectors. Once again this has proven a reliable method for sheeting with dove tailed and re-entrant profiles. The introduction of trapezoidal profiles of late has caused some concern about the methodology in which to assess the headed stud strength and ductility. The purpose of this paper is to attempt to address some of the issues associated with the shear stud behaviour consisting of trapezoidal profiles.

The strength and ductility of headed stud shear connectors of steel and concrete composite beams is normally determined from a standard push test as outlined by Eurocode 4. Figure 1 illustrates push test for solid and profiled slabs. The objective of the analysis was to carry out numerical push testing of similar sized specimens to the conventional Eurocode 4, British Standards Institution [1] push test. Back-breaking failures have been shown to be suppressed in numerical analyses and thus it is not considered necessary to model four ribs explicitly. When a push test is carried out, the shear connectors are subjected to pure shear, however in comparison, when a composite steel-concrete beam is subjected to bending, the shear connectors will be subjected to both bending and shear. 


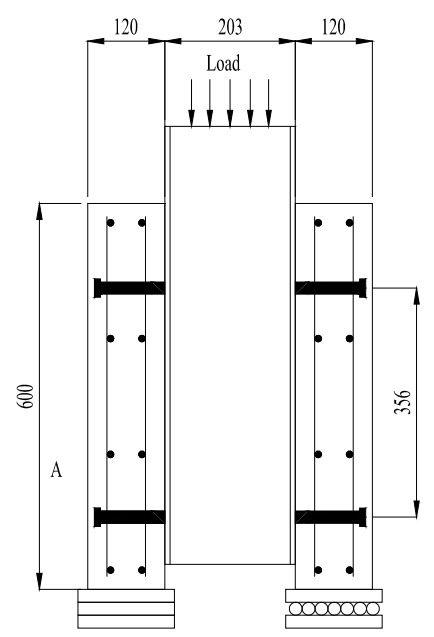

Solid Slab

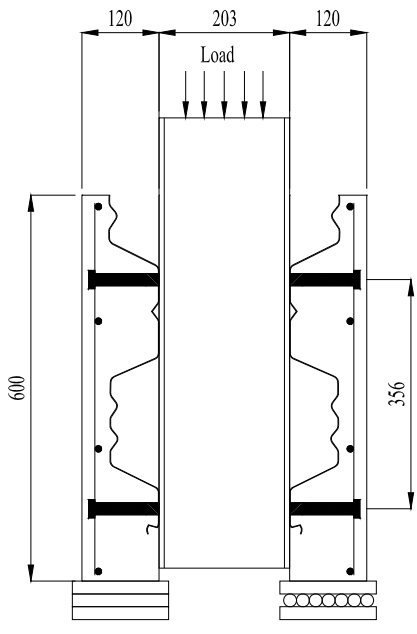

Profiled Slab

*All dimensions in mm

Figure 1. Push Tests for Both Solid and Profiled Slab

Even though headed stud shear connectors can resist a complex combination of shear, bending and axial force, the magnitudes of these forces change during a push test. Therefore the behaviour of headed stud shear connectors also depends greatly on the geometry of the push test specimens and the strength of all the materials. In this study, the authors have used pure shear as a way of identifying the applied load to the specimens in order to address the real stress state of headed stud shear connectors that suppress secondary failure modes and thereby simulate the real situation in beams illustrated by Hicks [2].

For a simplified explanation, consider the push test with a horizontal axis of symmetry, shown in Figure 2. Profiled slab in Figure 2 has been modified to illustrate the realistic stresses and thus the effects of torque on the ribs are modelled and Figure 13 also highlights the complex stress states created by this modelling approach. A horizontal line of the cross-section at the interface of the concrete slab and structural steel beam will be referred to as the neutral axis of the composite steel-concrete beam. Next consider a typical element of the composite beam between two planes perpendicular to the beam's neutral axis. From an elevation, the element is denoted as aabb. When the element is subjected to loading in the web of the structural steel beam, shown in Figure $2 \mathrm{a}$, it will be pushed as illustrated in Figure 2b. However section $a a b b$ still remains undeformed. Therefore, the structure is in pure shear where critical loading conditions are applied to the shear connectors in composite steel-concrete beams. In this case, the strain will remain constant along the section, as shown in Figure 2c.

When a composite steel-concrete beam is subjected to bending moments, consider the beam with a vertical axis of symmetry given in Figure 3. The horizontal line of the cross-section is similar to the push test explained above. When the element is subjected to loading on the concrete slab shown in Figure $3 \mathrm{a}$, the structure will bend as illustrated in Figure $3 \mathrm{~b}$, and then the section $c c d d$ deforms to become c'c'd'd'. Thus, the cross-section will be subjected to bending. For this case, the strain gradually changes throughout the depth of the section as illustrated in Figure 3c.

This paper considers the behaviour of strain regimes for the concrete element. As discussed above, when the structure is subjected to pure shear, the longitudinal strain is constant throughout the section, however when it is subjected to pure bending, the strain gradually changes throughout the depth of the section. A linear variation in strain is schematically shown in Figure 4, and the value of strain is represented using a parameter defined as $\alpha$. When the structure is subjected to pure shear, 
the strain distribution is represented by Figure $4 \mathrm{a}$ where $\alpha=1$. For the case when the structure is subjected to pure bending, the strain distribution is represented by Figure $4 \mathrm{e}$ where $\alpha=-1$. Figs. 4b, $4 \mathrm{c}$ and $4 \mathrm{e}$ illustrate the different variation of strain which represents the different locations of the neutral axis.

In order to modify the strain regimes in a concrete element from pure shear to pure bending, the theory of elasticity of Hooke's law according to Popov and Balan [4] was used. Authors considered the cross-section of the composite steel-concrete beam is deformed by a parallel system of small forces, $F_{i}$ in the axial direction as shown in Figure 2. A combination of the two concepts above will enable the axial force acting in every cross-section to be determined.

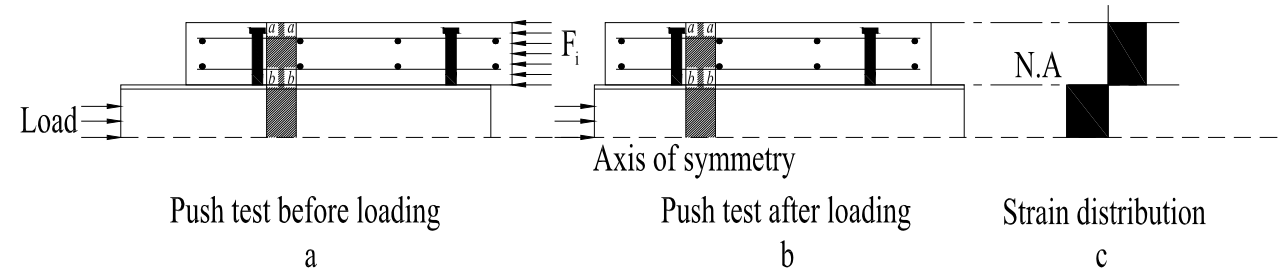

b

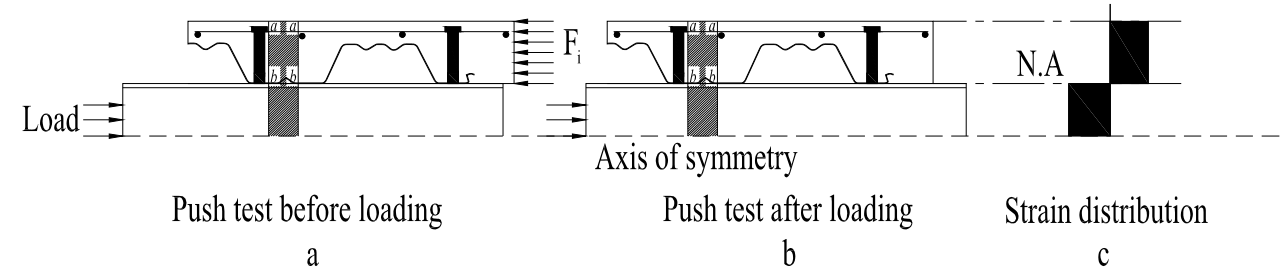

Figure 2. Behaviour of Composite Steel-Concrete Beams in Pure Shear

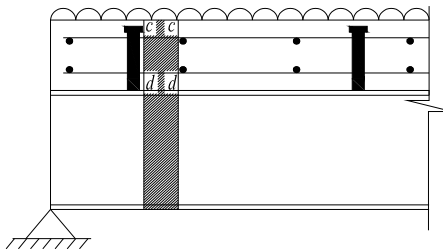

Beam before loading

a

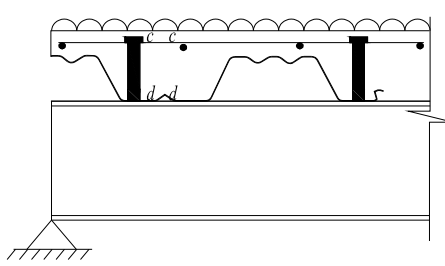

Beam before loading

a

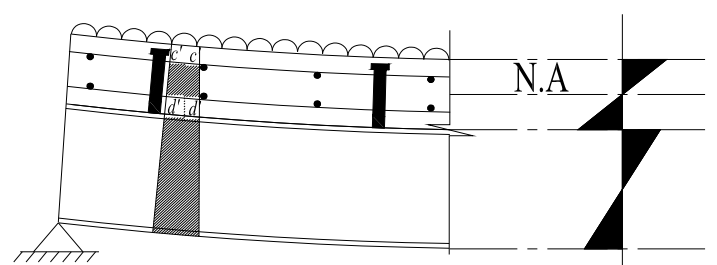

Beam after loading

b

Strain distribution

c

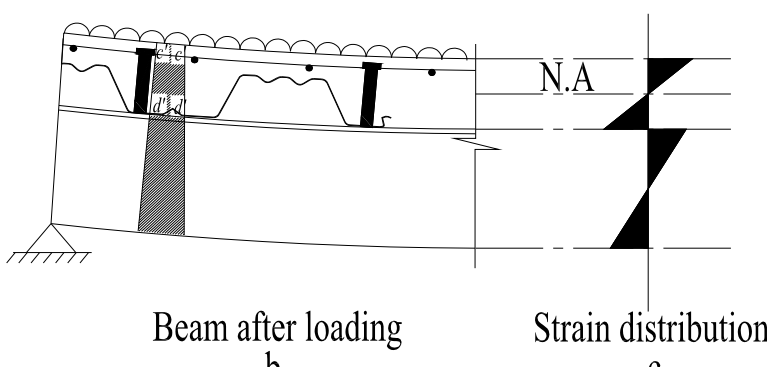

b

C

Figure 3. Behaviour of Composite Steel-Concrete Beams in Pure Bending 


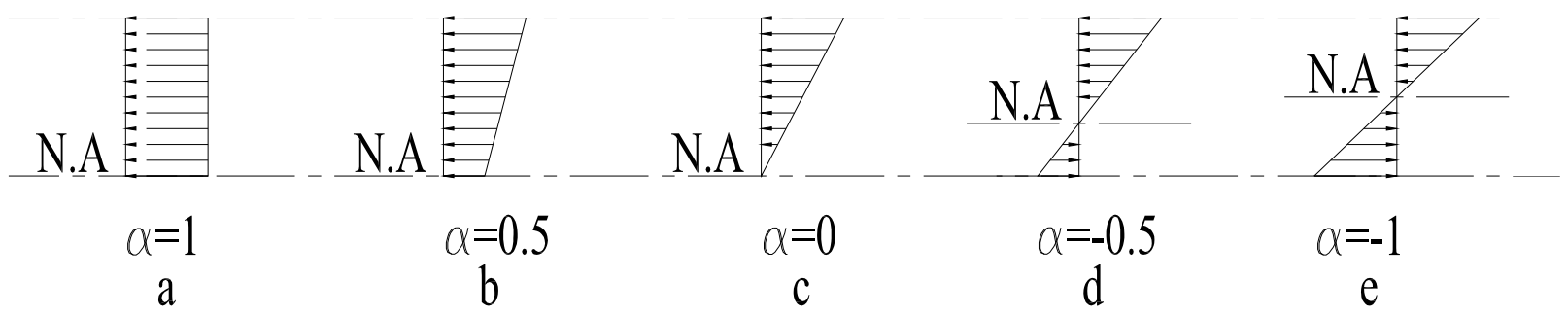

Figure 4. The Value of $\alpha$ and Location of Neutral Axis for the Concrete Element

\section{MECHANICAL BEHAVIOUR OF THE CONSTITUENT MATERIALS}

\section{$2.1 \quad$ General}

In general, constitutive laws are used to define the stress-strain characteristics of the material. The accuracy of the analysis is dependent on the constitutive laws used to define the mechanical behaviour. In materials such as concrete, structural steel and reinforcing steel, profiled steel sheeting and shear connectors, the constitutive laws are represented by the stress-strain relationships of the materials.

\subsection{Concrete Properties}

Plain concrete was recommended by Carreira and Chu [5], where the stress in compression is assumed to be linear with respect to strain up to a stress of $0.4 f^{\prime}{ }_{c}$. Beyond this point, the stress is represented as a function of strain according to Eq. 1.

$\sigma_{c}=\frac{f^{\prime}{ }_{c} \gamma\left(\varepsilon_{c} / \varepsilon_{c}^{\prime}\right)}{\gamma-1+\left(\varepsilon_{c} / \varepsilon_{c}^{\prime}\right)^{\gamma}}$

where

$\gamma=\left|\frac{f^{\prime}{ }_{c}}{32.4}\right|^{3}+1.55$ and $\quad \varepsilon^{\prime}{ }_{c}=0.002$

For concrete in tension, the tensile stress is assumed to increase linearly relative to strain until the concrete cracks. After the concrete cracks, the tensile stress decreases linearly to zero. The value of strain at zero stress is usually taken to be 10 times the strain at failure, which is illustrated in Figure 5.

\subsection{Structural Steel, Reinforcing Steel, Shear Connectors and Profiled Steel Sheeting Properties}

The stress-strain characteristics of reinforcing steel, shear connectors and profiled steel sheeting are essentially similar to structural steel. Their behaviour is initially elastic after which yielding and strain hardening develop. A piecewise linear approach was found to be sufficiently accurate to represent the stress-strain relationships. Moreover, these curves are utilised in the model when the stress-strain data is not available. 


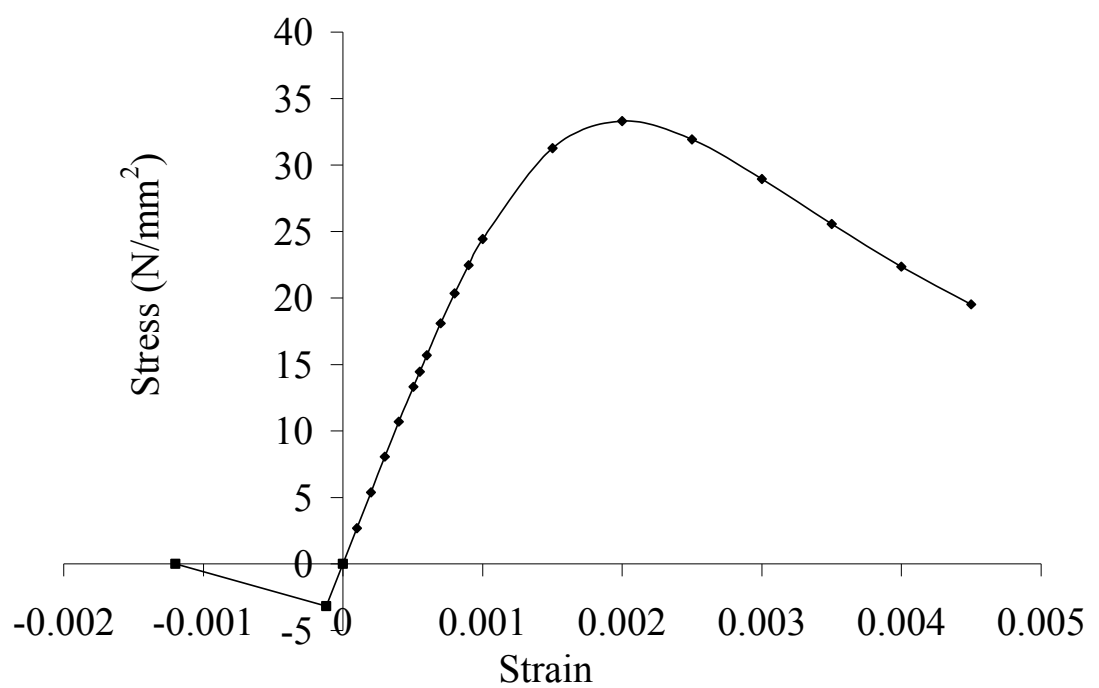

Figure 5. Stress-Strain Relationship for Concrete, Carreira and Chu [5]

According to Loh, et al. [6], the stress-strain relationship for structural steel can be represented as a simple elastic-plastic model with strain hardening. The mechanical behaviour for both compression and tension is assumed to be similar. Figure 6 represents the stress-strain relationship for steel and Table 1 indicates the different values of stress and strain for each material.

Table 1. Stress-Strain Value for Structural Steel Beam, Shear Connectors, Profiled Steel Sheeting and Steel Reinforcing

\begin{tabular}{|c|c|c|c|}
\hline Element & $\sigma_{u s}$ & $\varepsilon_{p s}$ & $\varepsilon_{u s}$ \\
\hline Steel beam & $1.28 \sigma_{y s}$ & $10 \varepsilon_{y s}$ & $30 \varepsilon_{y s}$ \\
\hline Steel Reinforcing & $1.28 \sigma_{y s}$ & $9 \varepsilon_{y s}$ & $40 \varepsilon_{y s}$ \\
\hline Profiled Sheeting & - & $20 \varepsilon_{y s}$ & - \\
\hline Shear Connectors & - & $25 \varepsilon_{y s}$ & - \\
\hline
\end{tabular}

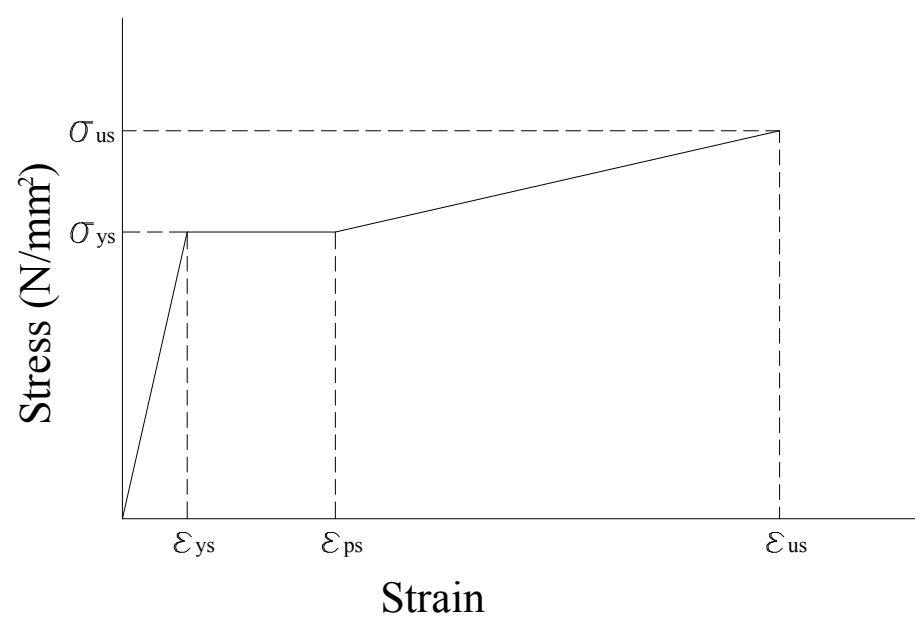

Figure 6. Stress-Strain Relationship for Structural Steel, Loh, et al. [6] 


\section{PUSH TEST FINITE ELEMENT ANALYSIS}

\subsection{Experimental Investigation of the Push Tests.}

Initially, there are two different experimental investigations which were compared with the finite element analysis developed herein. The push tests performed by Lam and El-Lobody [7] considered shear connection of both solid and profiled slabs. The authors modified Lam's solid slab to consider a profiled slab in order to evaluate the differences between the solid and profiled slabs. With the modified profiled slab, the authors only took into account the presence of the shear stud in the middle position of the shear connectors in the profiled sheeting. Mirza and Uy [8] stated that the strength of the shear connectors can be increased by $11 \%$ when they are placed on the strong side when compared with the weak position. Conversely when the shear connectors were positioned on the weak side, the strength of the shear connectors displayed a reduction of $13 \%$. The reader should bare in mind that the results shown are only applicable to the range of parameters studied herein. Qualification needs to be made against the range of parameters such as the thickness of the steel sheeting, shape of trough sheeting, arrangement and number of headed stud shear connectors in the trough as stated by Johnson and Yuan [9] and Johnson and Yuan [10] Further numerical analysis of these parameters could prove useful in the future.

The authors also considered the experiments conducted by Hicks [2]for both the push and beam tests as a comparison with the finite element analysis for the profiled slabs. For these analyses, the authors used the shear studs that were placed in the favourable position as it was adopted in the experimental program.

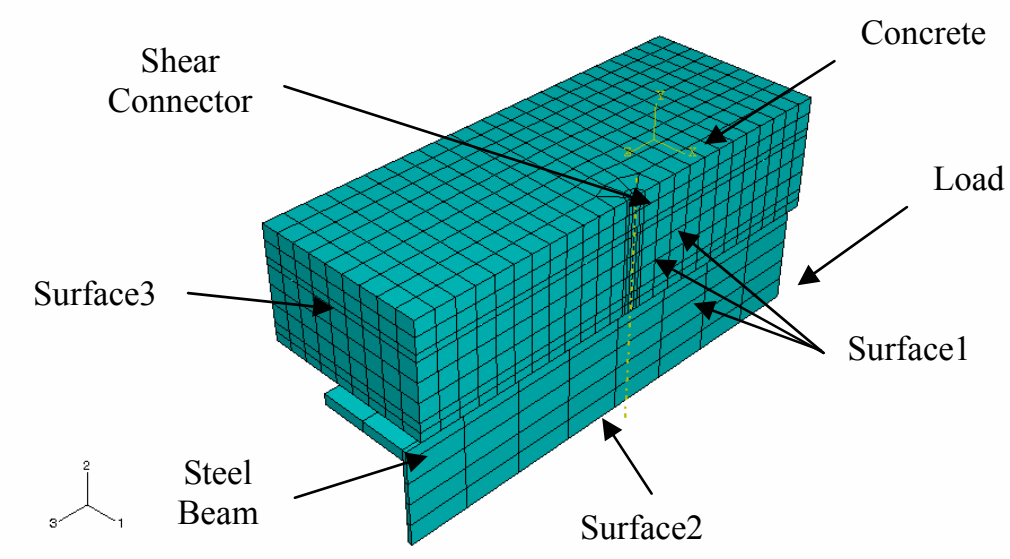

Figure 7. Finite Element Mesh and Boundary Condition of Solid Slab Model, Lam and El-Lobody [7] 


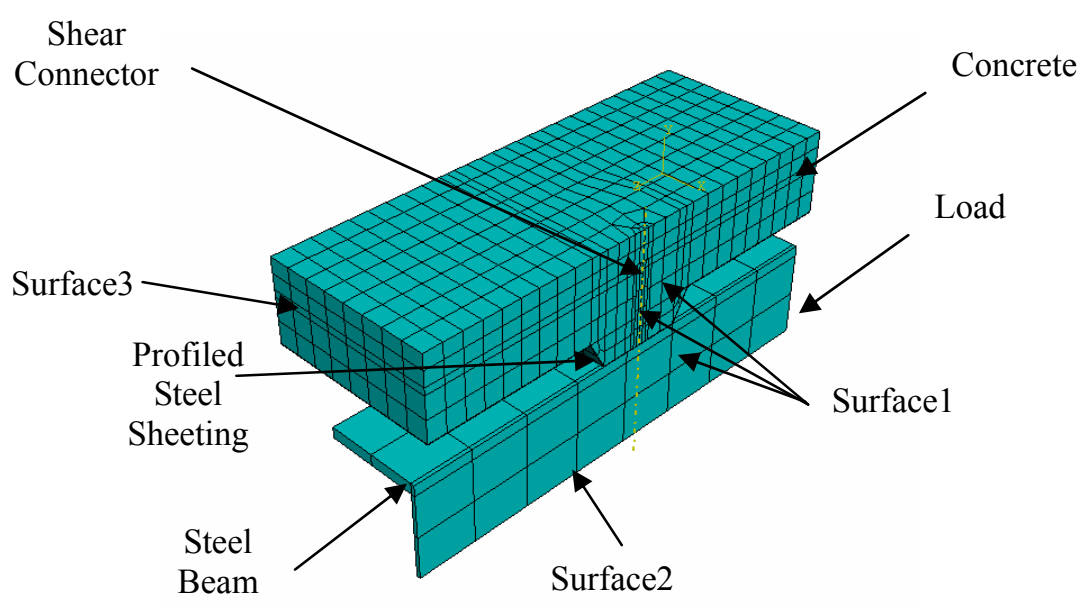

Figure 8. Finite Element Mesh and Boundary Condition of Profiled Steel Sheeting Slab Model, Lam and El-Lobody [7]

\subsection{Parametric Studies for the Push Tests.}

The authors also undertook parametric studies by considering different types of profiled steel sheeting and their effects on the shear connector behaviour when different strain regimes were considered. Three different types of profiled steel sheeting were considered, and they include G1, G2 and G3 profiled steel decks. The dimensions and profiled steel sheeting details are illustrated in Table 2.

\subsection{Finite Element Type and Mesh}

The finite element program ABAQUS, Karlsson and Sonrensen [11], Karlsson and Sonrensen [12] and Karlsson and Sonrensen [13] were used to quantify the behaviour of the shear connection in composite beams. Three dimensional solid elements were used to model the push off test specimens in order to achieve an accurate result from the finite element analysis. For both the concrete slab and the structural steel beam, a three-dimensional eight node element (C3D8R) was used to improve the rate of convergence. A three dimensional thirty-node quadratic brick element (C3D20R) for the shear connectors was chosen due to their ability to capture stress concentrations more effectively and better for modelling geometric features. Furthermore a four-node doubly curved thin shell element (S4R) for the profiled steel sheeting because it is the most appropriate type of element to model thin walled steel structures and lastly a two-node linear three dimensional truss element (T3D2) for the steel reinforcing was used because the axial direction is released using two node linear displacement equation provided in ABAQUS. The main reason was that the authors were not too concerned with the slip between the reinforcing bar and concrete.

For the experiments of Lam and El-Lobody [7], the finite element model was used to represent half of a stud of the push test, due to symmetrical conditions for both the solid and modified profiled slabs which are illustrated in Figs 7 and 8 respectively. Whilst Hicks [2] experiments, the finite element model used to represent half of the push test model is shown in Figure 9. The reason the authors used different models for Lam and El-Lobody [7] and Hicks [2]was to reduce the simulation cost. 
Table 2. Dimensions and Profiled Steel Sheeting Details of Parametric Study

\begin{tabular}{|c|c|c|c|c|c|c|c|c|c|}
\hline \multirow[t]{3}{*}{ Group } & \multirow[t]{3}{*}{ Specimen } & \multicolumn{7}{|c|}{ Dimensions } & \multirow{3}{*}{$\begin{array}{c}\text { Strain } \\
\text { Profiles } \\
\\
\alpha \\
\end{array}$} \\
\hline & & \multicolumn{3}{|c|}{ Profiled Sheeting } & \multicolumn{2}{|c|}{ Stud } & \multicolumn{2}{|c|}{ Slab } & \\
\hline & & $b_{0}(\mathrm{~mm})$ & $h_{p}(\mathrm{~mm})$ & $t(\mathrm{~mm})$ & $d(\mathrm{~mm})$ & $h(\mathrm{~mm})$ & $B(\mathrm{~mm})$ & $D(\mathrm{~mm})$ & \\
\hline \multirow[t]{5}{*}{ G1 } & G1-1 & 136 & 60.9 & 0.9 & 19 & 100 & 806 & 405 & 1 \\
\hline & G1-2 & 136 & 60.9 & 0.9 & 19 & 100 & 806 & 405 & 0.5 \\
\hline & G1-3 & 136 & 60.9 & 0.9 & 19 & 100 & 806 & 405 & 0 \\
\hline & G1-4 & 136 & 60.9 & 0.9 & 19 & 100 & 806 & 405 & -0.5 \\
\hline & G1-5 & 136 & 60.9 & 0.9 & 19 & 100 & 806 & 405 & -1 \\
\hline \multirow[t]{5}{*}{ G2 } & $\mathrm{G} 2-1$ & 136 & 55 & 0.9 & 19 & 100 & 806 & 405 & 1 \\
\hline & $\mathrm{G} 2-2$ & 136 & 55 & 0.9 & 19 & 100 & 806 & 405 & 0.5 \\
\hline & $\mathrm{G} 2-3$ & 136 & 55 & 0.9 & 19 & 100 & 806 & 405 & 0 \\
\hline & $\mathrm{G} 2-4$ & 136 & 55 & 0.9 & 19 & 100 & 806 & 405 & -0.5 \\
\hline & $\mathrm{G} 2-5$ & 136 & 55 & 0.9 & 19 & 100 & 806 & 405 & -1 \\
\hline \multirow[t]{5}{*}{ G3 } & G3-1 & 144 & 60.9 & 0.9 & 19 & 100 & 806 & 405 & 1 \\
\hline & G3-2 & 144 & 60.9 & 0.9 & 19 & 100 & 806 & 405 & 0.5 \\
\hline & G3-3 & 144 & 60.9 & 0.9 & 19 & 100 & 806 & 405 & 0 \\
\hline & G3-4 & 144 & 60.9 & 0.9 & 19 & 100 & 806 & 405 & -0.5 \\
\hline & G3-5 & 144 & 60.9 & 0.9 & 19 & 100 & 806 & 405 & -1 \\
\hline
\end{tabular}

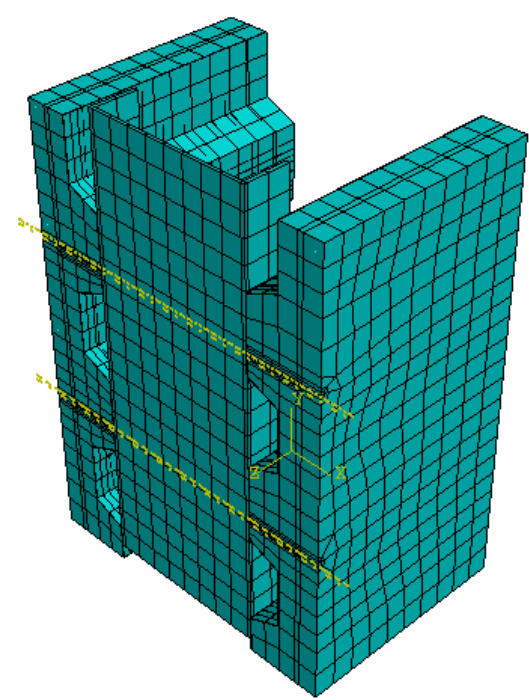

Figure 9. Finite Element Mesh and Boundary Condition of Profiled Steel Sheeting Slab Model, Hicks [2] 


\subsection{Boundary Conditions and Load Application}

In Figs. 7 and 8, the nodes that lie on the other symmetrical surface (Surface 1) for concrete, shear connectors, structural steel beam, steel reinforcing and profiled steel sheeting are restricted from moving in the $x$-direction. All the nodes in the middle of the structural steel beam web, which are designated as Surface 2, are restricted to move in the $y$-direction. All the nodes of the concrete and the profiled steel sheeting, which are designated Surface 3, are restricted to move in the $z$-direction. Whilst in Figure 9, Surface 3 which consists of a concrete slab, structural steel beam, steel reinforcing and profiled steel sheeting are restricted to move in the $z$-direction.

For the application of load, a static concentrated load was applied to the centre of the web for Lam's model [5] and a uniformly distributed load was applied to the web surface of the structural steel for the Hicks' model [7]. The modified RIKS method was employed to the load in order for the load to be obtained through a series of iterations for each increment for a non-linear structure. This method was used to predict unstable and nonlinear collapse of the model. In order to achieve accurate results in ABAQUS, the RIKS method has the ability to use the arc length along the static equilibrium in load-displacement space. The initial increments will be adjusted if the finite element model fails to converge. Finally the value of deformation after each increment is computed automatically. The final result will either be the maximum value of the load or the maximum displacement value.

\section{RESULTS AND DISCUSSION}

\subsection{Lam and El-Lobody's Experiments}

For the purpose of this paper, finite element push tests were modelled to determine the load-slip behaviour of the shear connectors. The first series of analyses considered was compared with the experimental investigations undertaken by Lam and El-Lobody [7] for solid slabs. The authors then modified the solid slab to simulate a profiled slab to consider the differences in the results.

It can be observed in Figure 10 that the stiffness is similar for both the experiments and the finite element analyses within the elastic region. The experimental study showed that the maximum shear connector capacity was $118 \mathrm{kN}$ whilst the finite element result obtained was $119 \mathrm{kN}$. This shows that the finite element model accurately analysed the experiments with a discrepancy of $0.7 \%$. When the model is subjected to bending where $\alpha=-1$, after the elastic region, the finite element analysis had a higher shear connector capacity when compared with the model subjected to pure shear where $\alpha=1$.

From the finite element model, the authors have also shown that when the composite steel-concrete beam is subjected to pure shear, the structure is subjected to the most detrimental loading case. This can be verified from Figure 10 where the model is subjected to bending, the shear connector capacity increased by $2.2 \%$ to $121 \mathrm{kN}$. Even though the loading behaviour of the push test changed, $2.2 \%$ of increment in the shear connector capacity is not significant. In order to look at the different behaviour of the shear connectors when exposed to different loading conditions with varying neutral axes, Figure 10 established that the different strain regimes in solid slabs is not critical where increments in $0 \%, 0.7 \%$ and $1.3 \%$ for $\alpha=0.5, \alpha=0$ and $\alpha=-0.5$ respectively. Therefore, it is illustrated that the push test to determine the shear connector capacity for composite steel-concrete solid slabs is reliable and accurate. 
From Figure 10, it is observed that the failure mode changes when the value of $\alpha$ changes. When $\alpha=1$, the failure mode is governed by shear stud failure. When $\alpha=0.5,0,-0.5$ and -1 , the failure mode is governed by concrete failure where the concrete starts to crack surrounding the shear connector due to the addition of load when the value of $\alpha$ changes. The failure modes can also be observed graphically in Figure 11.

Figure 12 illustrates that the stiffness is similar for the finite element analyses within the elastic region. The finite element analysis showed that the maximum shear connector capacity was $68 \mathrm{kN}$ when $\alpha=1$ for the push test under pure shear, however when the model is subjected to the bending condition where $\alpha=-1$, the maximum shear connector capacity was $92 \mathrm{kN}$. This shows a significant increment of $26.6 \%$. The result is expected when the push test changes from pure shear to pure bending due to the loading condition for the worst case scenario when compared with pure bending.

Figure 12 illustrates that the different strain regimes in the profiled slab are crucial where an increment in $6.8 \%, 12.5 \%$ and $18.5 \%$ for $\alpha=0.5,0$ and -0.5 respectively. Therefore, it is illustrated that the push test to determine the shear connector capacity for composite steel-concrete profiled slabs is unreliable as it does not allow for the inclusion of the beneficial effects of bending.

From Figure 12, it can be seen that the failure mode changes when the value of $\alpha$ changes. When $\alpha=1$ and 0.5 , the failure mode is governed by concrete failure where the concrete cracks through the middle of the trough. This failure mode observed is similar to experimental studies undertaken by El-Lobody and Young [14]. When $\alpha=0,-0.5$ and -1 , the failure mode is dominated by stud failure where the shear connectors started to shear off the concrete, causing the concrete to crack surrounding the shear connector. The failure mode also can be observed in Figure 13.

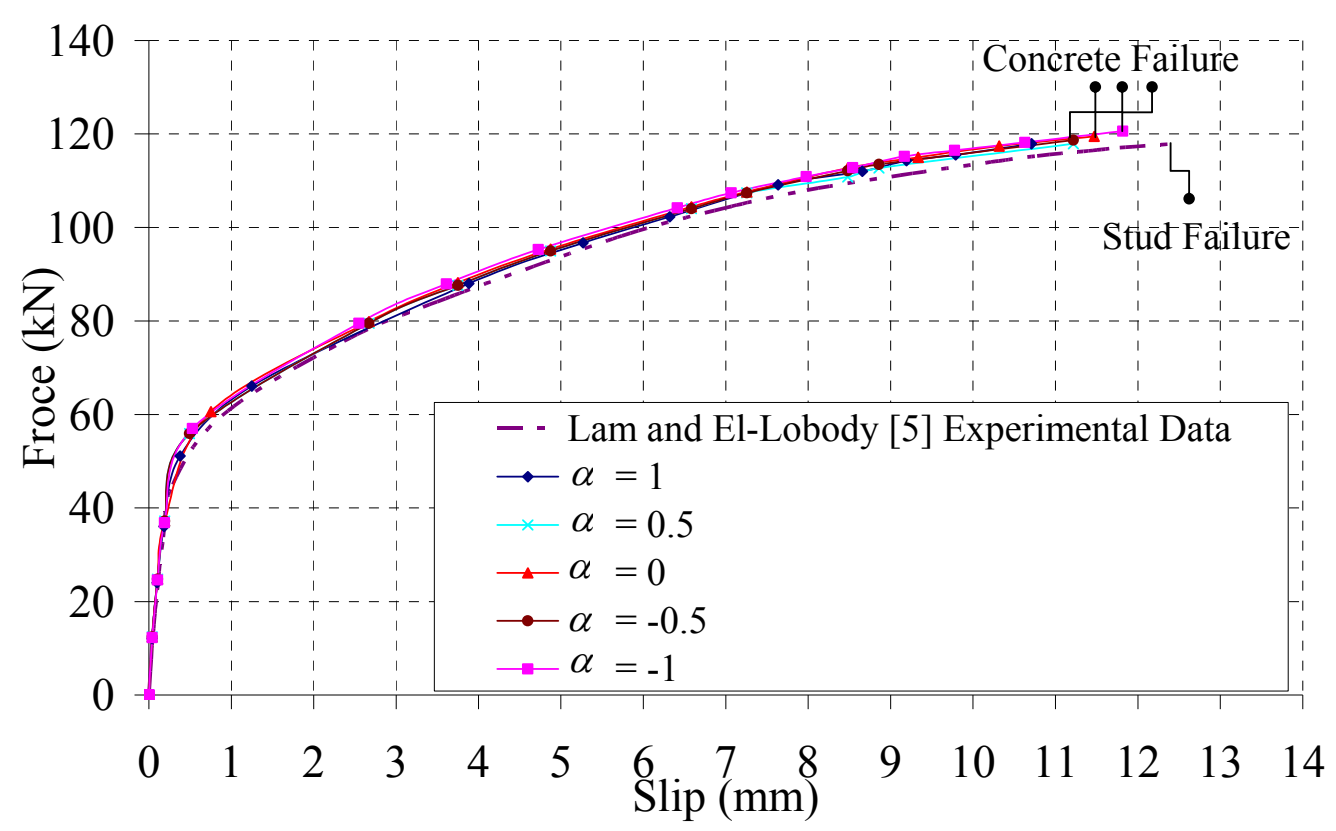

Figure 10. Lam and El-Lobody [5] Experiment Compared with Different Strain Regimes 


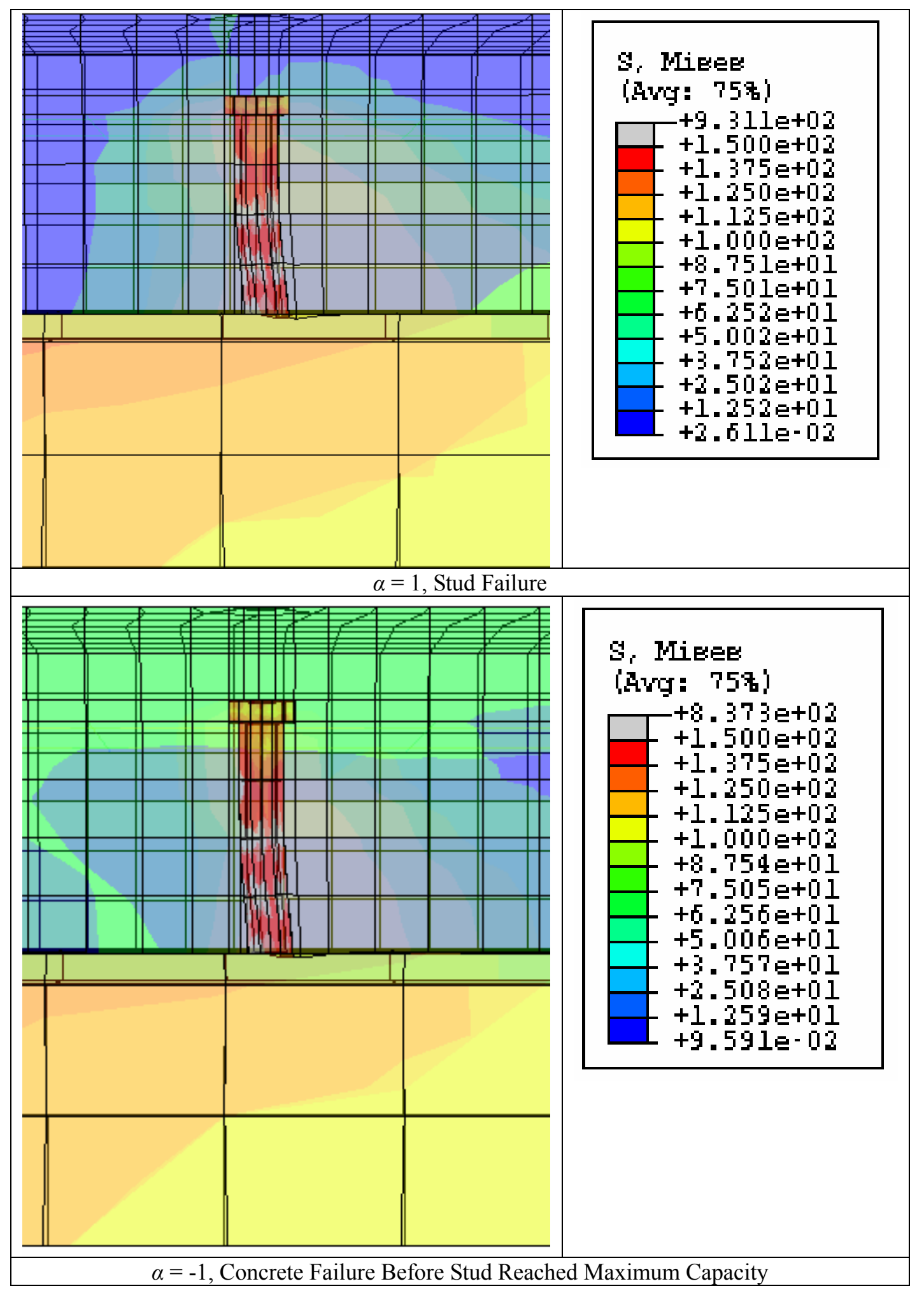

Figure 11. Stress Contours and Failure Modes for Solid Slabs 


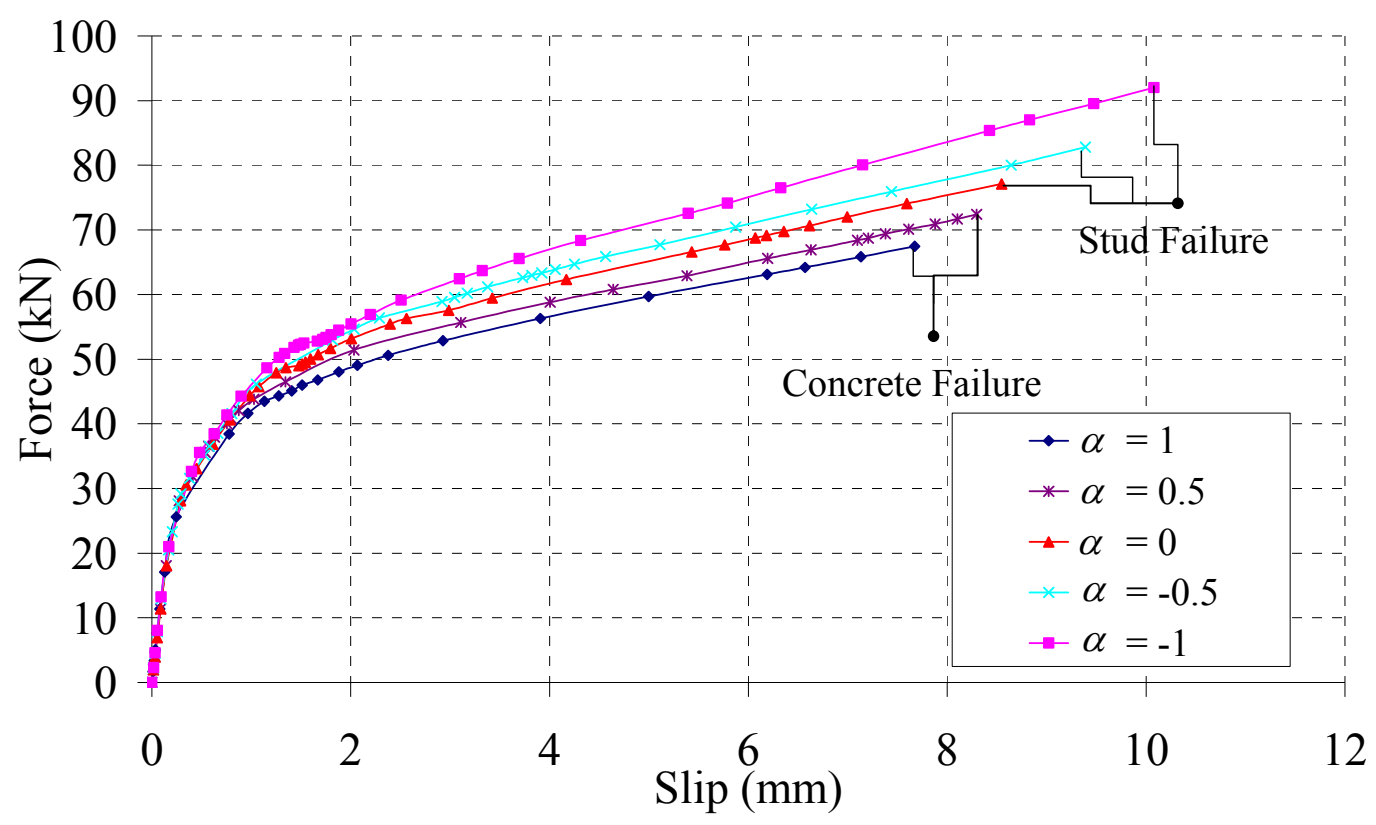

Figure 12. Lam and El-Lobody’s Modified Push Test with Different Strain Regimes

\subsection{Hicks' Experiments}

The second experimental series considered was that associated with the experiments conducted by Hicks [2]for both push tests and beam tests. Figure 14 shows the comparison between the experimental tests undertaken by Hicks' and the finite element model performed by the authors. The results are as stated by Hicks [2], the initial similarity is the stiffness for both the push test and beam test. However, the maximum shear connector capacity and slip measured in the push test is well below the level attained in the beam test. Figure 14 verifies the accuracy of the finite element analysis. For the push tests, the experimental study showed a maximum shear capacity of $84 \mathrm{kN}$ and the finite element analysis showed a value of $89 \mathrm{kN}$ with a discrepancy of $5.7 \%$. As for the beam tests, the experimental study and finite element analysis illustrated that the maximum shear capacities were $124 \mathrm{kN}$ and $128 \mathrm{kN}$ respectively, with a discrepancy of $3.3 \%$.

Furthermore, Figure 14 shows that the maximum shear connector capacity for push tests was $89 \mathrm{kN}$, whilst for the beam tests, the maximum shear connector capacity was $128 \mathrm{kN}$. This illustrates an increment of $30.6 \%$. From the experimental studies and finite element analyses, failure in the push tests was governed by concrete failure at the trough rather than stud failure. When the simulated beam test is considered, the failure is dominated by stud failure. The behaviour of the shear connectors in the push test provides a conservative result and they do not replicate the strength and ductility that can be achieved in a beam test. Therefore, the standard push test to determine the shear connector capacity for a profiled slab is questionable. 


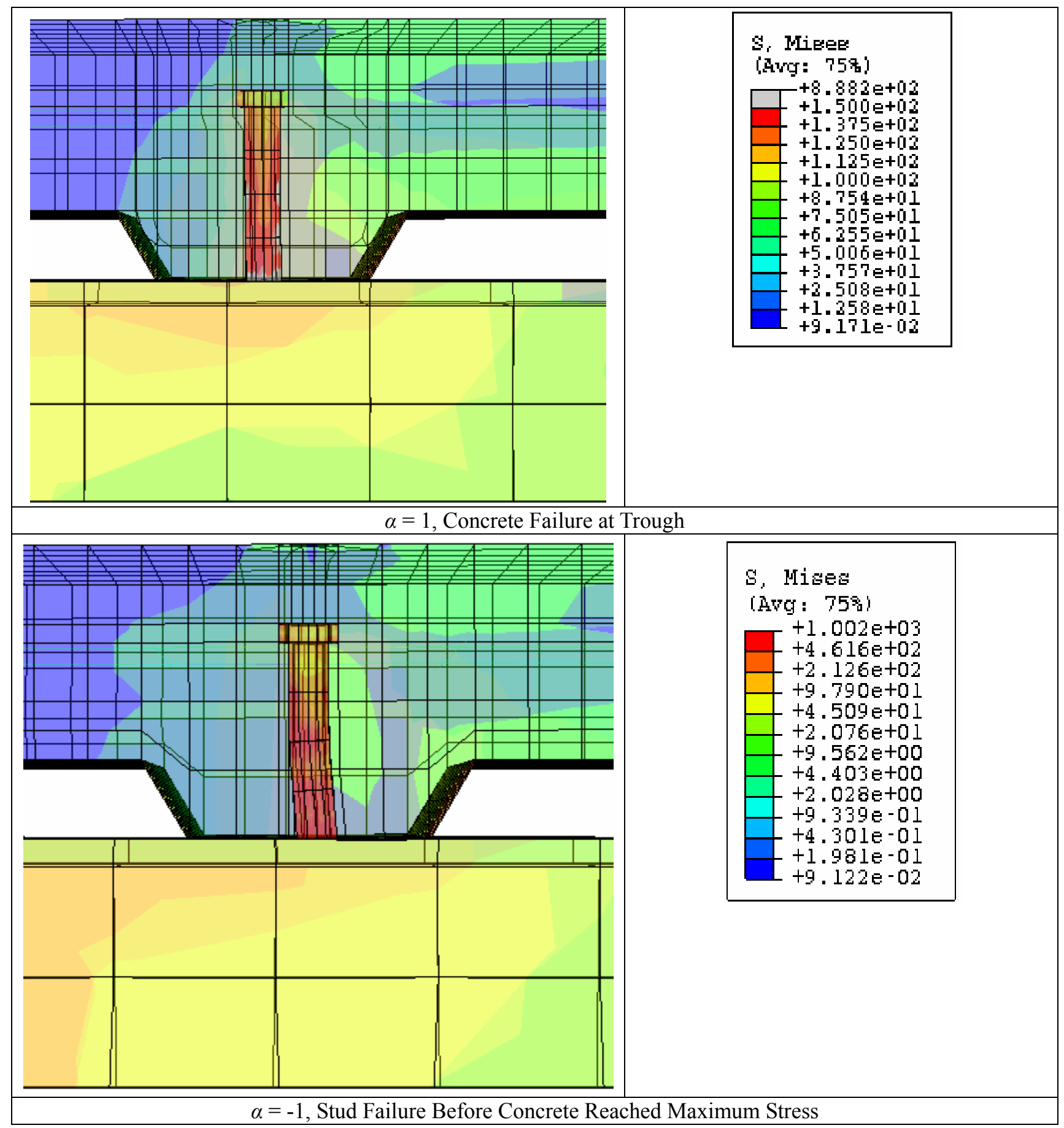

Figure 13. Stress Contours and Failure Modes for Profiled Slabs 


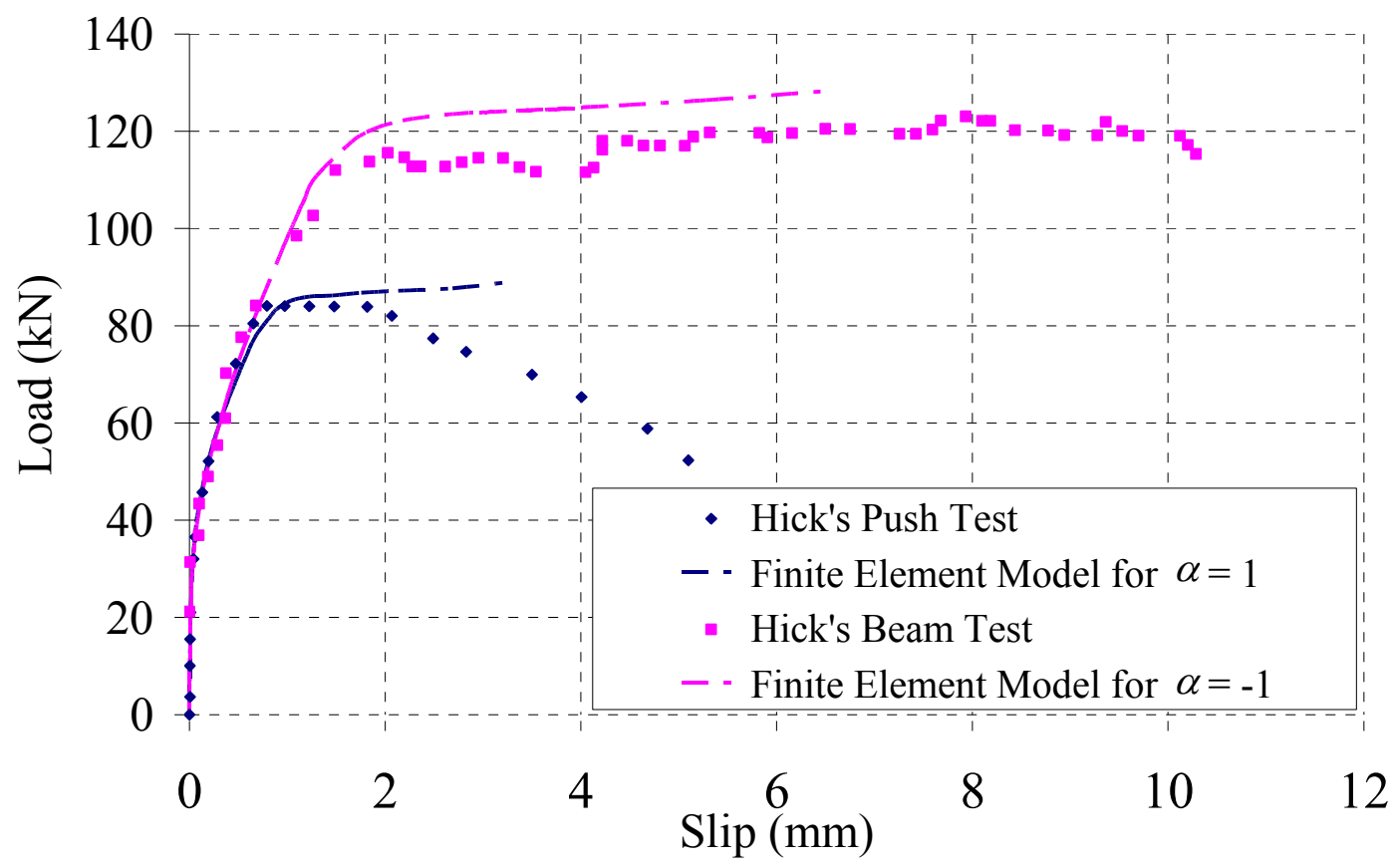

Figure 14. Comparison of Experimental Data and Finite Element Result for Hicks [2]

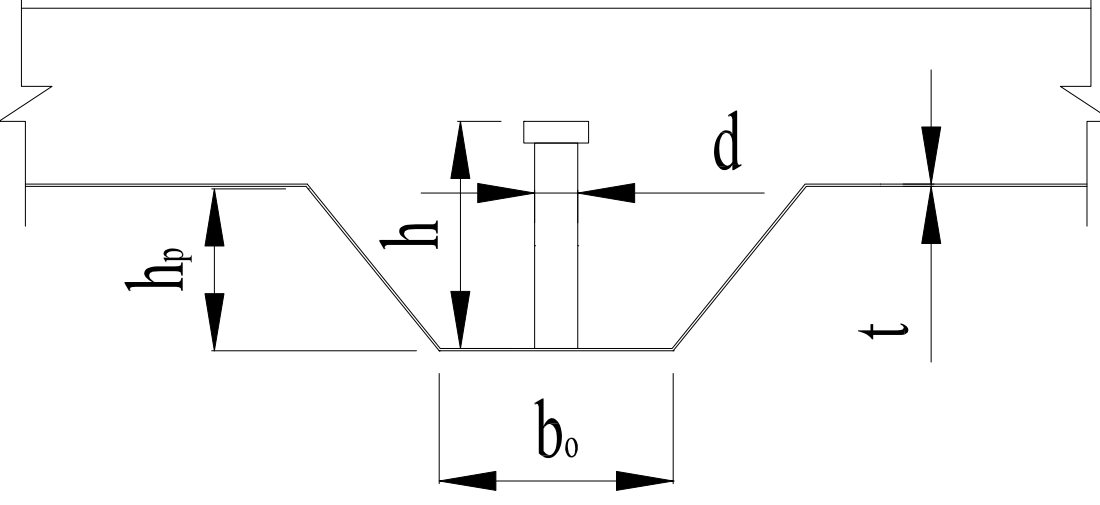

Figure 15. Dimensions of Profiled Steel Sheeting 


\subsection{Parametric Studies}

\subsubsection{General}

The finite element analysis has allowed parametric studies to be carried out and the case that the authors solved has been modelled particularly in calibration with independent experimental test results undertaken by Hicks [2]. This may not be the case for real buildings. However, the trends in this paper may be of use for further studies. In this case, it has been shown that the finite element model correctly predicts the behaviour of shear connectors in composite steel-concrete structures for both solid and profiled slabs. For solid slabs, when the strain regimes were varied from pure shear to pure bending, the maximum capacity did not show any critical change, whilst for profiled slabs, any minor change was crucial. Hence, a parametric study was conducted to study the effects of the capacity and behaviour of shear connectors by changing the profiled steel sheeting geometries. A total of fifteen push tests were investigated in the parametric study. The push tests were divided into three major groups. These were G1, G2 and G3. Each group included five different push tests having different $\alpha$ values with the same type of profiled steel sheeting, as described in Figure 4. The dimensions of the profiled steel sheeting and details of the parametric study are explained in Table 2 and shown in Figure 15.

Figure 16 illustrates the force-slip relationship for profile G1, G2 and G3 profiled steel sheeting types. It can be seen that between the three profiled steel sheeting types, G1 profiled steel sheeting can withstand larger forces when compared with the other two sheeting types. Therefore, the G1 type has a higher strength capacity and is considerably more ductile. Figure 16 reveals a maximum shear connector capacity of $135 \mathrm{kN}, 125 \mathrm{kN}$ and $128 \mathrm{kN}$ for G1, G2 and G3 profiled steel sheeting respectively. G1 illustrates that it has a shear connector capacity $4.8 \%$ higher than that of the G3 trapezoidal profiled sheeting and $7.3 \%$ higher than that of the G2 profiled steel sheeting. Figure 16 also shows that the shear strength capacity of composite steel-concrete profiled slabs is greatly dependent on the width and depth of the ribs of the profiled steel sheeting.

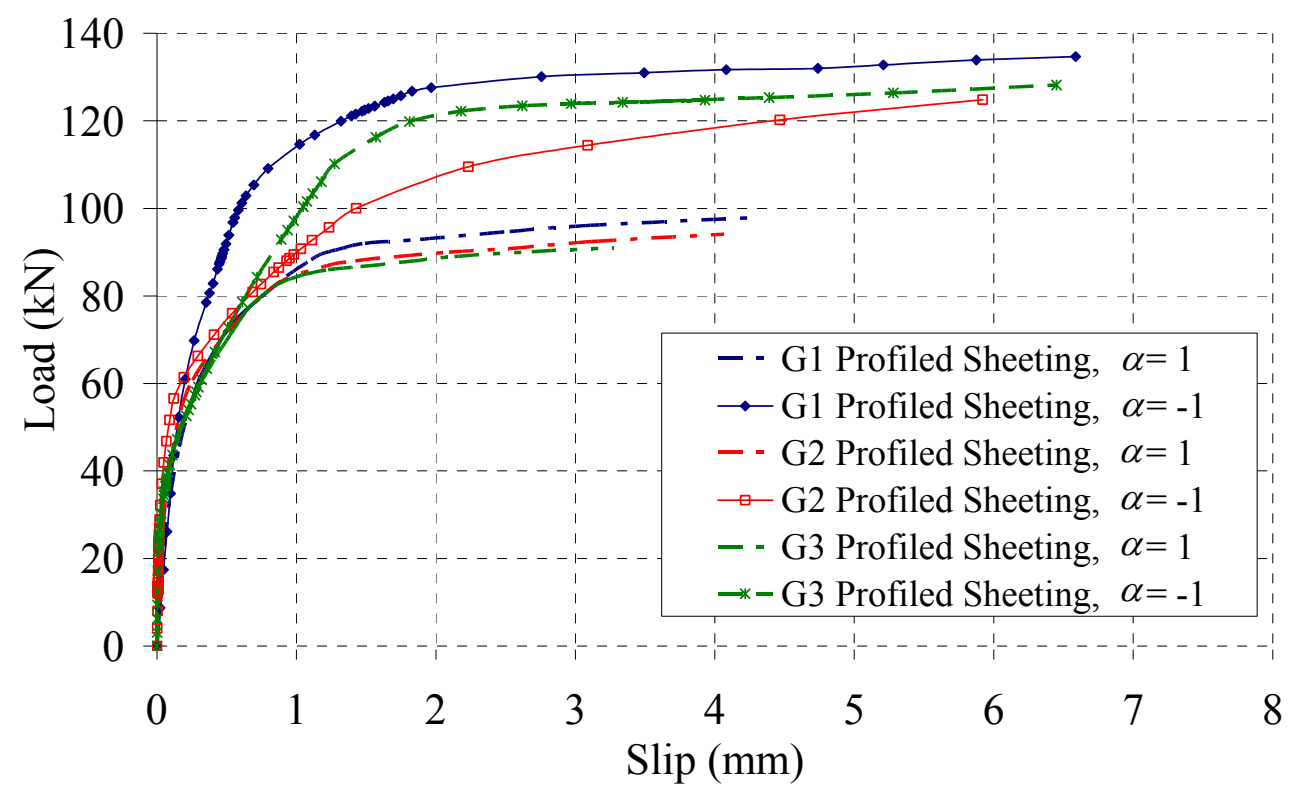

Figure 16. Comparison of Force Slip Relationship for Different Type of Profiled Steel Sheeting 


\subsubsection{G1 profiled}

The first group of parametric studies, the authors varied the strain regimes from $\alpha=1,0.5,0,-0.5$ and -1 for the G1 profiled steel sheeting. Figure 17 depicts the force-slip relationship of the G1 profiled steel sheeting with the variation of $\alpha$ value. It can be observed that the composite steel concrete beam increases the shear strength and ductility when the structure is varied from pure shear to pure bending. When the structure is subjected to pure shear, the maximum shear connector capacity of $98 \mathrm{kN}$ is achieved. However, when it is subjected to pure bending, the maximum shear capacity increases to $135 \mathrm{kN}$, which is a $27.2 \%$ shear capacity increment.

In order to add the $\alpha$ value into the force-slip relationship, the Aribert and Labib [15] force-slip relationship was used which is described in Eq. 2.

$Q=Q_{u}\left(1-e^{-c_{1} \cdot d}\right)^{c_{2}}$

where

$Q$ is the force of the shear connector

$Q_{u}$ is the ultimate force

$c_{1}$ and $c_{2}$ are the parameters of the model

$d$ is the slip capacity of the shear connector

Due to $Q_{u}, c_{1}$ and $c_{2}$ are the changing parameters in the equation, the relationship of these values with respect to $\alpha$ and is shown in Eq. 3. Each of the parameter values with respect to $\alpha$ can be obtained in Table 3 and plotted in Figures 18 to 20.

$Q=f_{1}(\alpha)\left(1-e^{-f_{2}(\alpha) \cdot d}\right)^{f_{3}(\alpha)}$

Table 3. Parameter of Force Slip Relationship with Respect to $\alpha$.

\begin{tabular}{|c|c|c|c|c|c|c|c|c|c|c|c|c|c|c|}
\hline \multirow{2}{*}{$\begin{array}{l}\text { Profiled } \\
\text { Type }\end{array}$} & \multicolumn{4}{|c|}{$f_{1}(\alpha)=a \alpha^{3}+b \alpha^{2}+c \alpha+d$} & \multicolumn{5}{|c|}{$f_{2}(\alpha)=e \alpha^{4}+f \alpha^{3}+g \alpha^{2}+h \alpha+j$} & \multicolumn{5}{|c|}{$f_{3}(\alpha)=k \alpha^{4}+l \alpha^{3}+m \alpha^{2}+n \alpha+p$} \\
\hline & $a$ & $b$ & $c$ & $d$ & $e$ & $f$ & $g$ & $h$ & $j$ & $k$ & $l$ & $m$ & $n$ & $p$ \\
\hline G1 & & & -17.94 & 117.2 & & -0.06 & 0.13 & 0.12 & 1.2 & -0.07 & -0.03 & 0.17 & 0.03 & 0.4 \\
\hline $\mathrm{G} 2$ & 13.30 & -1.51 & -28.60 & 111.5 & & 0.15 & 0.15 & 0.35 & 0.96 & 0.02 & -0.07 & -0.03 & 0.07 & 0.4 \\
\hline G3 & & & -18.36 & 109.74 & 0.67 & 0.2 & 0.27 & 0.3 & 1.05 & -0.07 & -0.03 & 0.17 & 0.03 & 0.4 \\
\hline
\end{tabular}

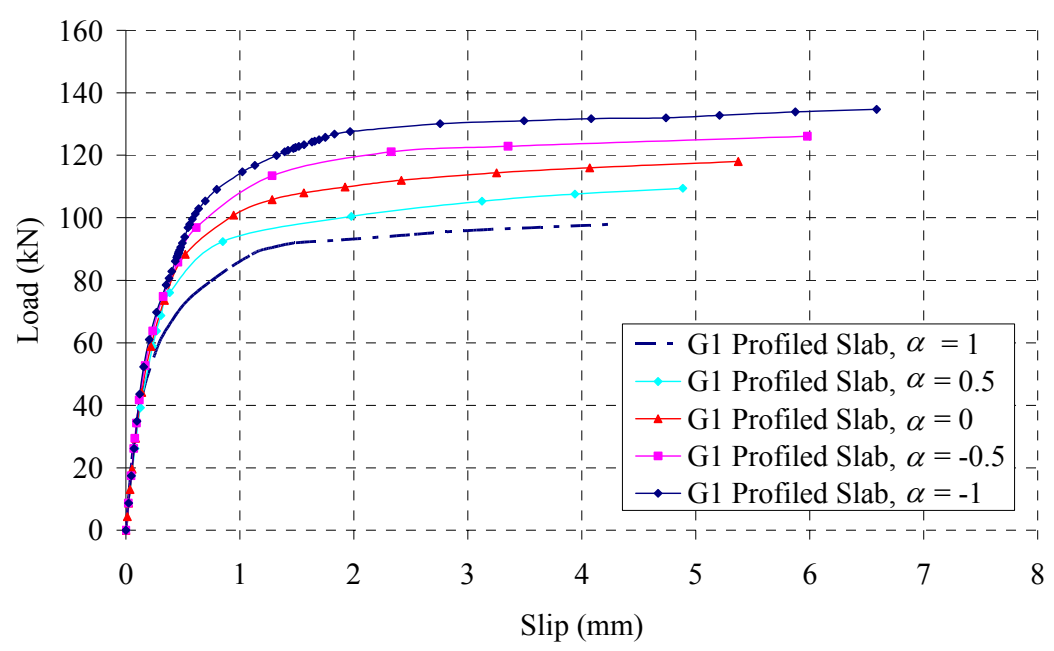

Figure 17. Force Slip Relationship for G1 Profiled Steel Sheeting with Variation of $\alpha$ Value 


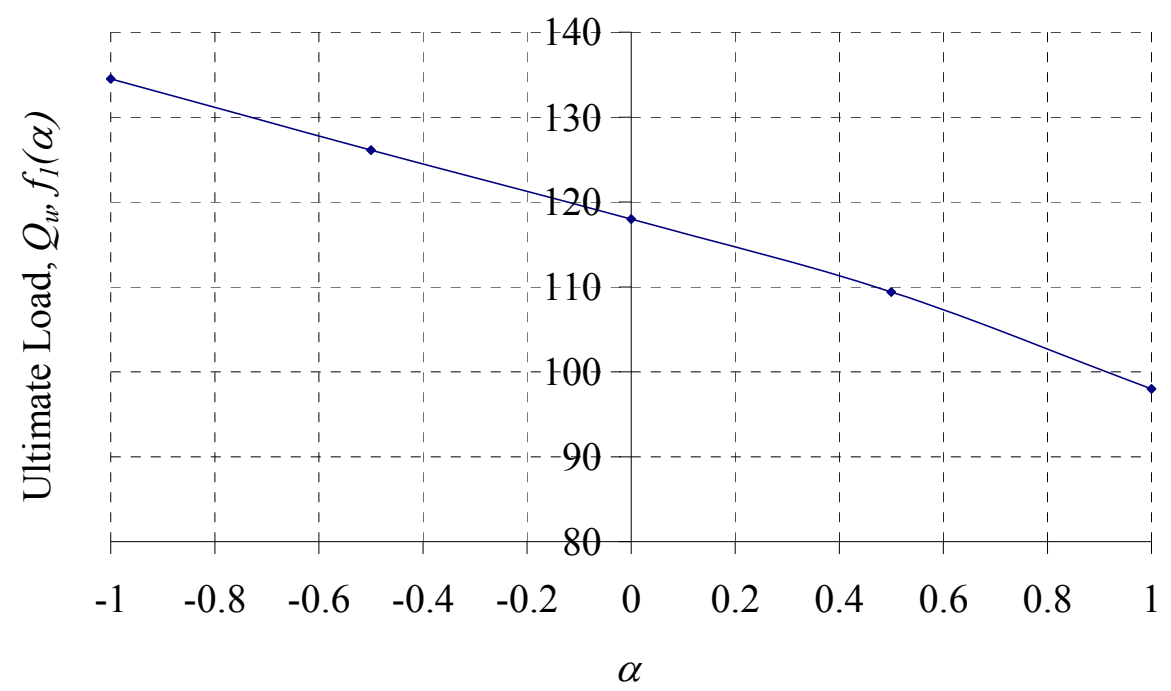

Figure 18. Ultimate Load with Respect to $\alpha$ for G1 Profiled Steel Sheeting

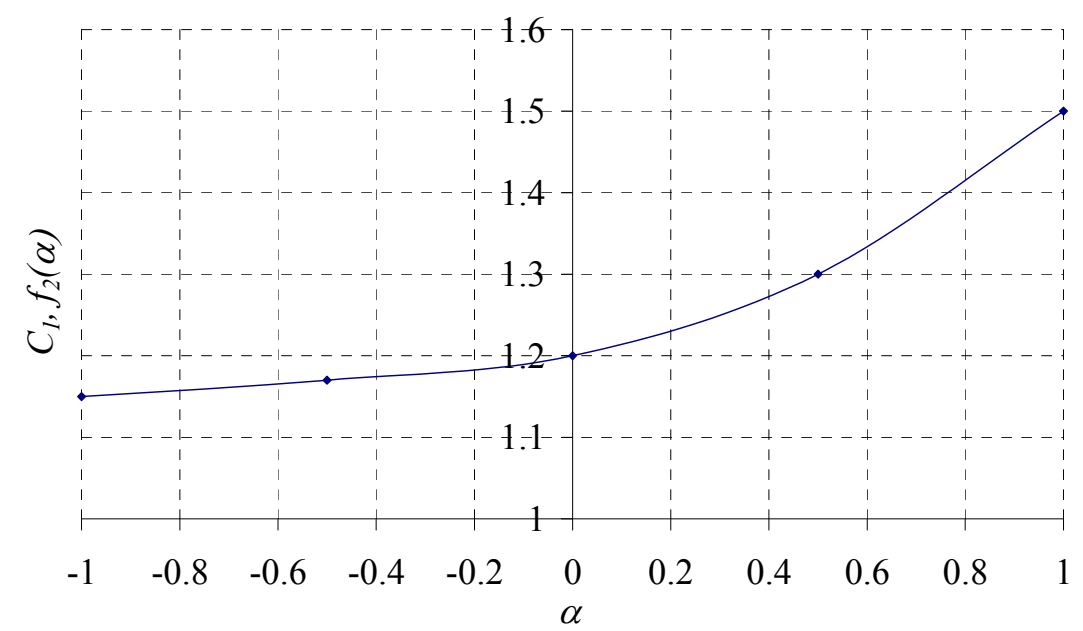

Figure 19. $c_{1}$ with Respect to $\alpha$ for G1 Profiled Steel Sheeting

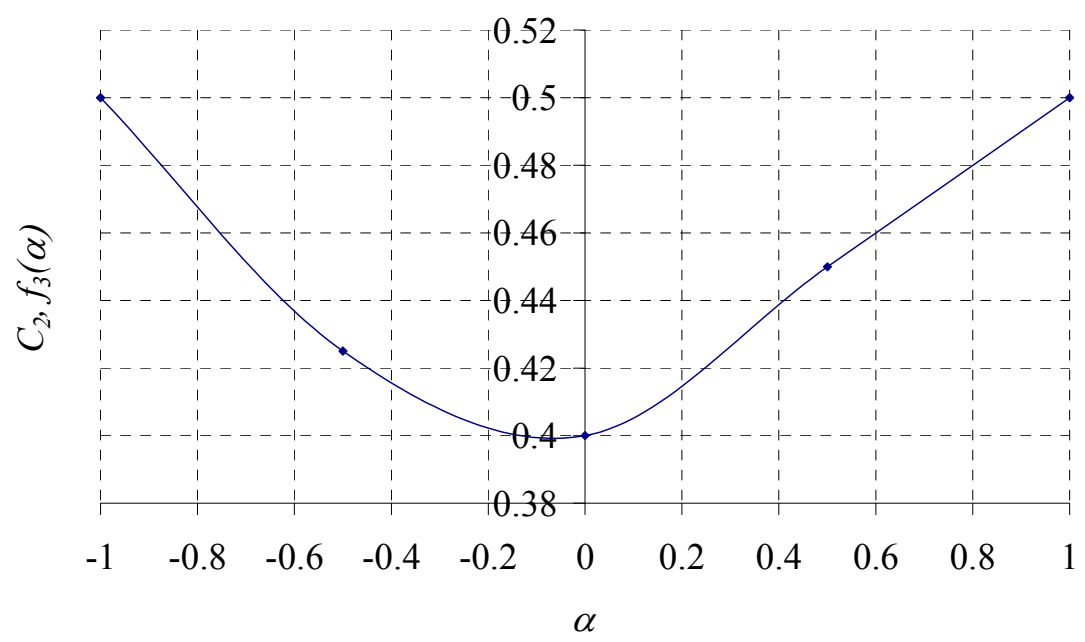

Figure 20. $c_{2}$ with Respect to $\alpha$ for G1 Profiled Steel Sheeting 


\subsubsection{G2 profiled}

The second group of parametric studies, the authors varied the strain regimes which included $\alpha=1$, $0.5,0,-0.5$ and -1 for G2 profiled steel sheeting. Figure 21 shows the force-slip relationship of G2 profiled steel sheeting with the variation of $\alpha$ value. The observation of the shear strength and ductility is similar to that mentioned in Section 4.3.2 above. When the structure is subjected to pure shear and pure bending, the maximum shear connector capacity is $94 \mathrm{kN}$ and $125 \mathrm{kN}$ respectively, which is a $25 \%$ shear capacity increment. The definition of $f_{1}, f_{2}$ and $f_{3}$ with respect to $\alpha$ is determined in Table 3 and plotted in Figures 22 to 24.

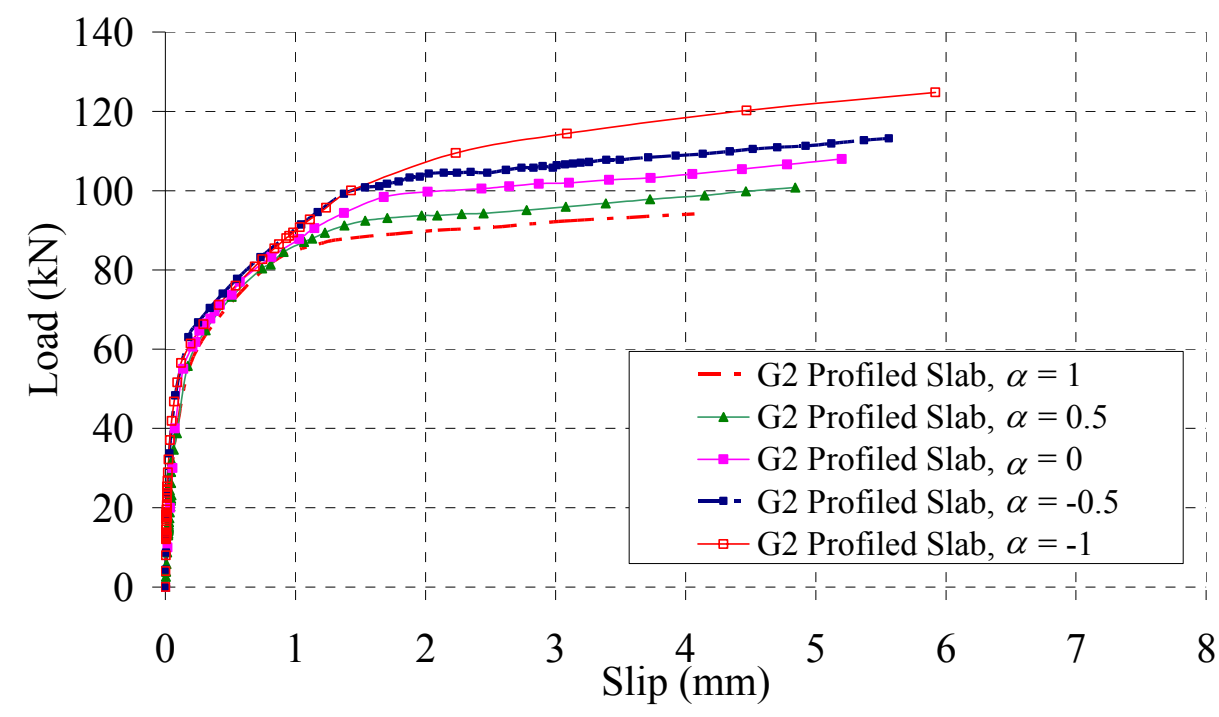

Figure 21. Force Slip Relationship for G2 Profiled Steel Sheeting with Variation of $\alpha$ Values

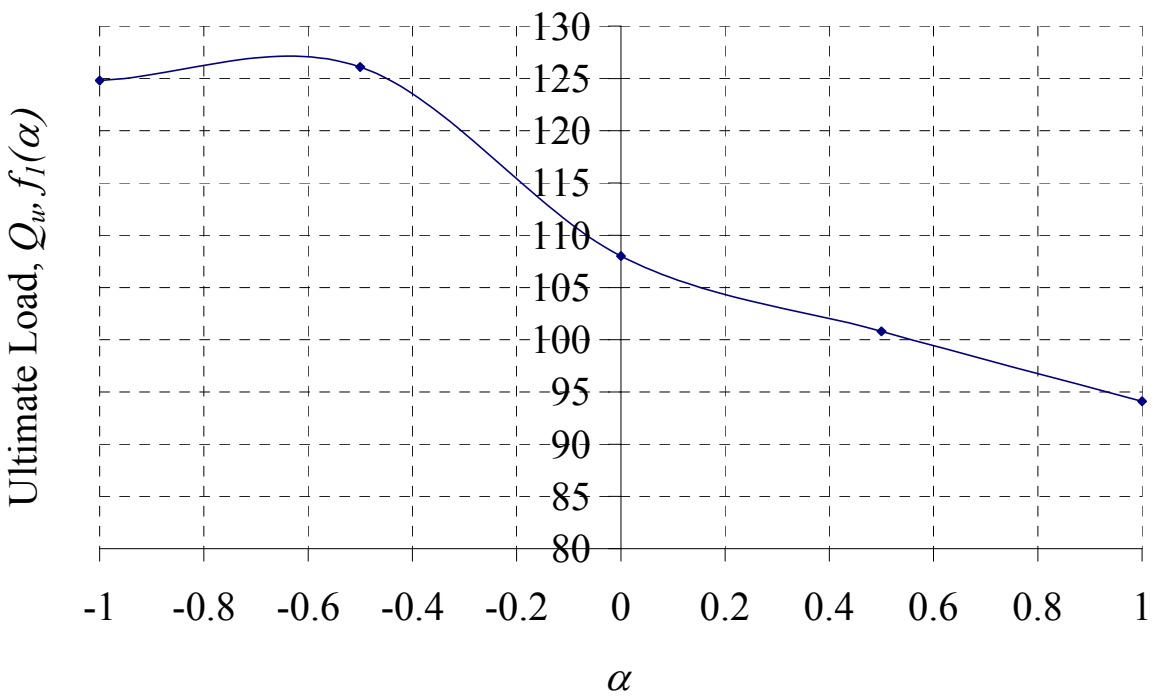

Figure 22. Ultimate Load with Respect to $\alpha$ for G2 Profiled Steel Sheeting 


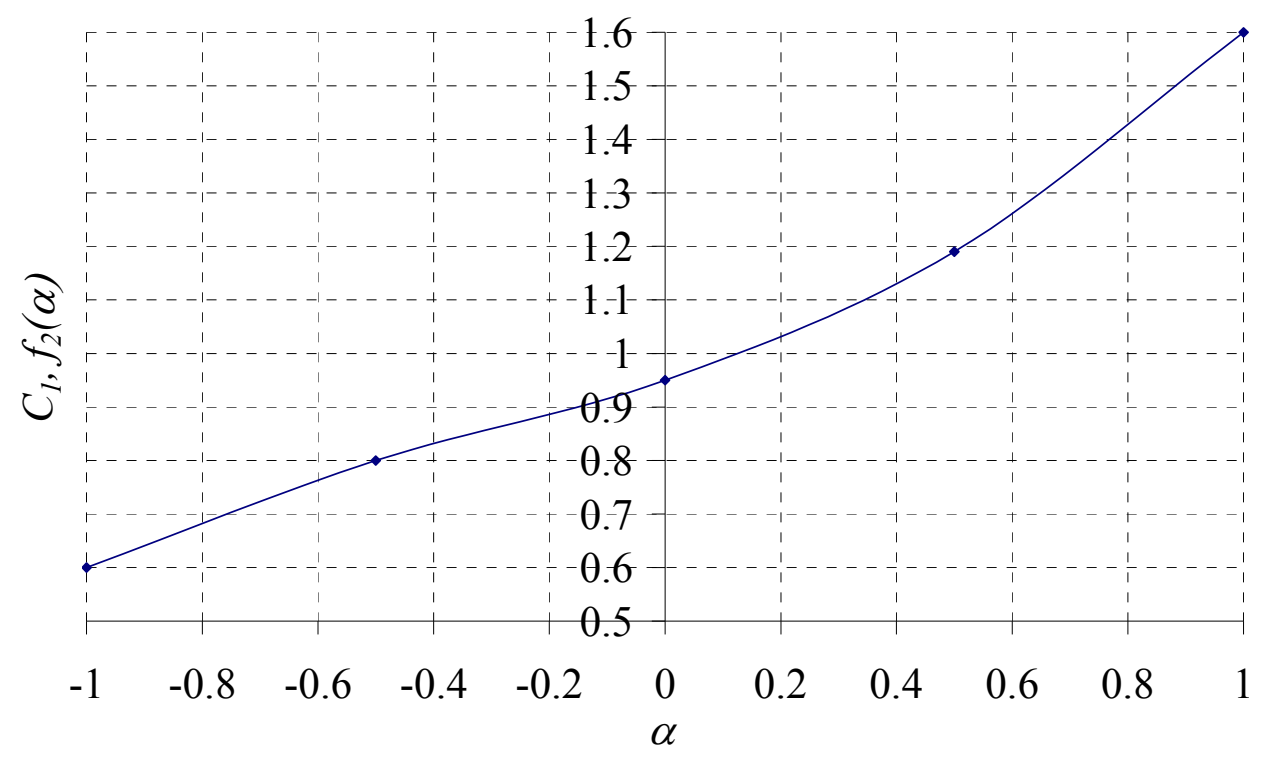

Figure 23. $c_{1}$ with Respect to $\alpha$ for G2 Profiled Steel Sheeting

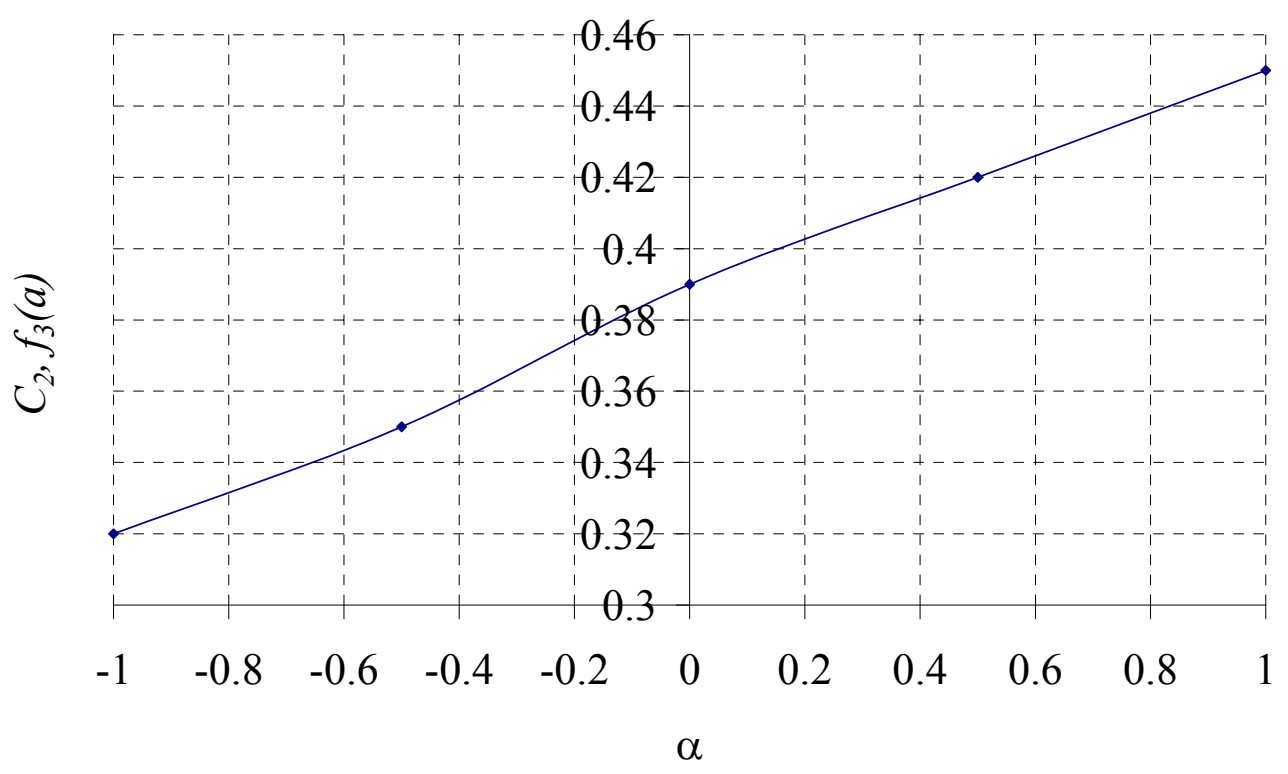

Figure 24. $c_{2}$ with Respect to $\alpha$ for G2 Profiled Steel Sheeting 


\subsubsection{G3 profiled}

The third group of parametric studies, G3 is used to verify Hicks' trapezoidal profiled steel sheeting. Figure 25 illustrates the force-slip relationship of G3 trapezoidal profiled steel sheeting with the variation of $\alpha$ value. The results illustrate that the shear strength and ductility is similar as mentioned in Section 4.3.2 above. When the structure is subjected to pure shear and pure bending, the maximum shear connector capacity is $91 \mathrm{kN}$ and $128 \mathrm{kN}$ respectively which is a $29 \%$ increase in shear capacity. The definition of $f_{1}, f_{2}$ and $f_{3}$ with respect to $\alpha$ is determined in Table 3 and plotted in Figures 26 to 28.

An example of Hicks [2] experimental study was used to verify the accuracy of these equations. Figure 29 shows the cross-section through the beam, and from Figure 29, the calculation to determine the $\alpha$ value is as below:

$f^{,}{ }_{c}=28 \mathrm{~N} / \mathrm{mm}^{2}$

$f^{\prime}{ }_{y}=378 \mathrm{~N} / \mathrm{mm}^{2}$

$N_{\text {concrete }}=0.85 f_{c}^{\prime} B D_{n}$

$N_{\text {concrete }}=0.85(28)(2500)(80)$

$N_{\text {concrete }}=4760 \mathrm{kN}$

$N_{\text {steel }}=2 N_{\text {flange }}+N_{\text {web }}$

$N_{\text {steel }}=2 b_{f} d_{f} f_{y}^{\prime}+b_{w} d_{w} f_{y}^{\prime}$

$N_{\text {steel }}=2(10.4)(167)(378)+7.3(289.2)(378)$

$N_{\text {steel }}=2111 \mathrm{kN}$

From the above equations,

$N_{\text {concrete }}>N_{\text {steel }}$

Therefore the neutral axis, N.A. falls in the concrete element.

Compression, $\mathrm{C}=$ Tension, $\mathrm{T}$

$0.85(28)(2500) d_{n}=2111$

$d_{n}=35.5 \mathrm{~mm}$

In order to find the value of $\alpha$

$\alpha=\frac{d_{n}}{D}$

$\alpha=-\frac{35.5}{140-35.5}$

$\alpha=-0.34$

From Figures 26 to 28 , the $Q_{u}, c_{1}$ and $c_{2}$ can be determined and the force-slip relationship is derived as shown in Eq. 4.

$Q=115.98\left(1-e^{-0.92 . d}\right)^{0.41}$

Eq. 4 is plotted in Figure 30, and from Figure 30, it is verified that when the correct $\alpha$ value of -0.34 is determined, the discrepancy of the result is only $0.3 \%$ when compared with the initial assumption of $\alpha=-1$ where the discrepancy is $4 \%$. 


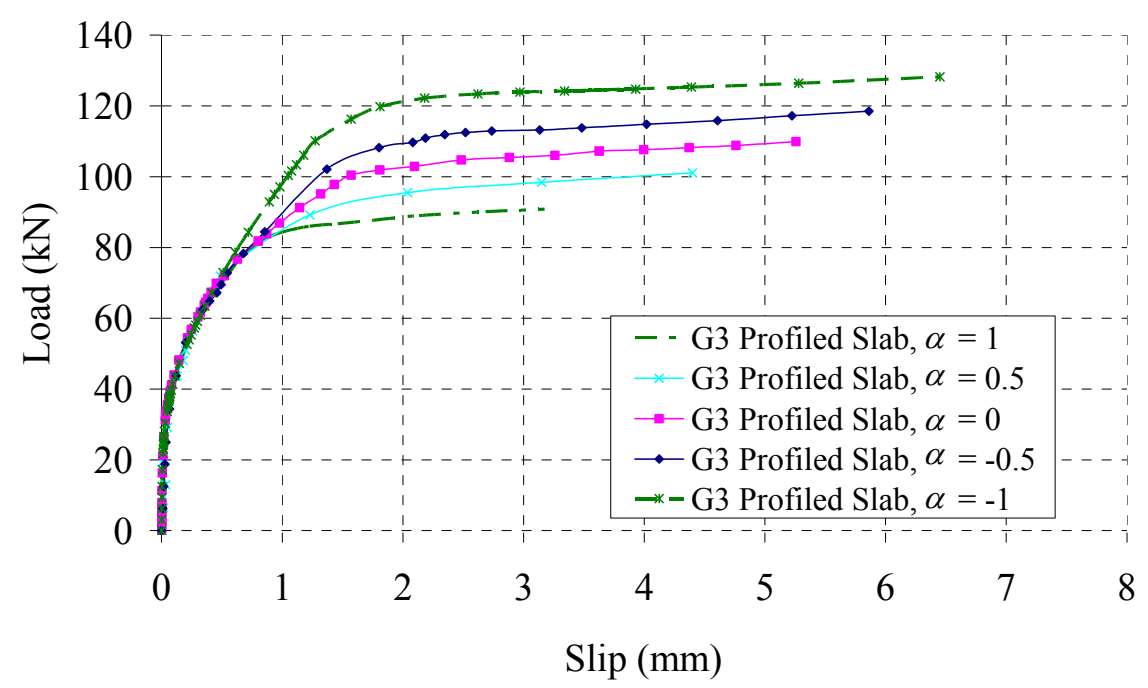

Figure 25. Force Slip Relationship for G3 Profiled Steel Sheeting with Variation of $\alpha$

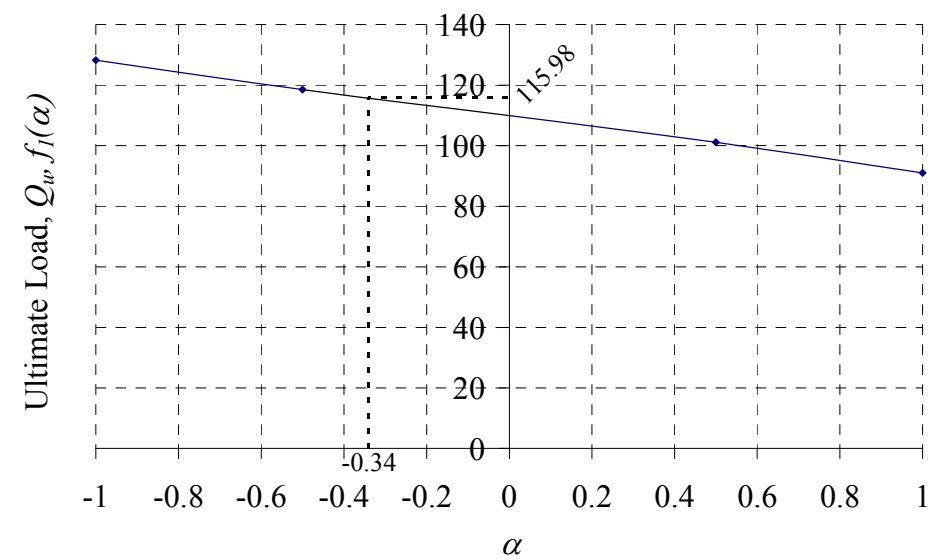

Figure 26. Ultimate Load with Respect to $\alpha$ for G3 Profiled Steel Sheeting

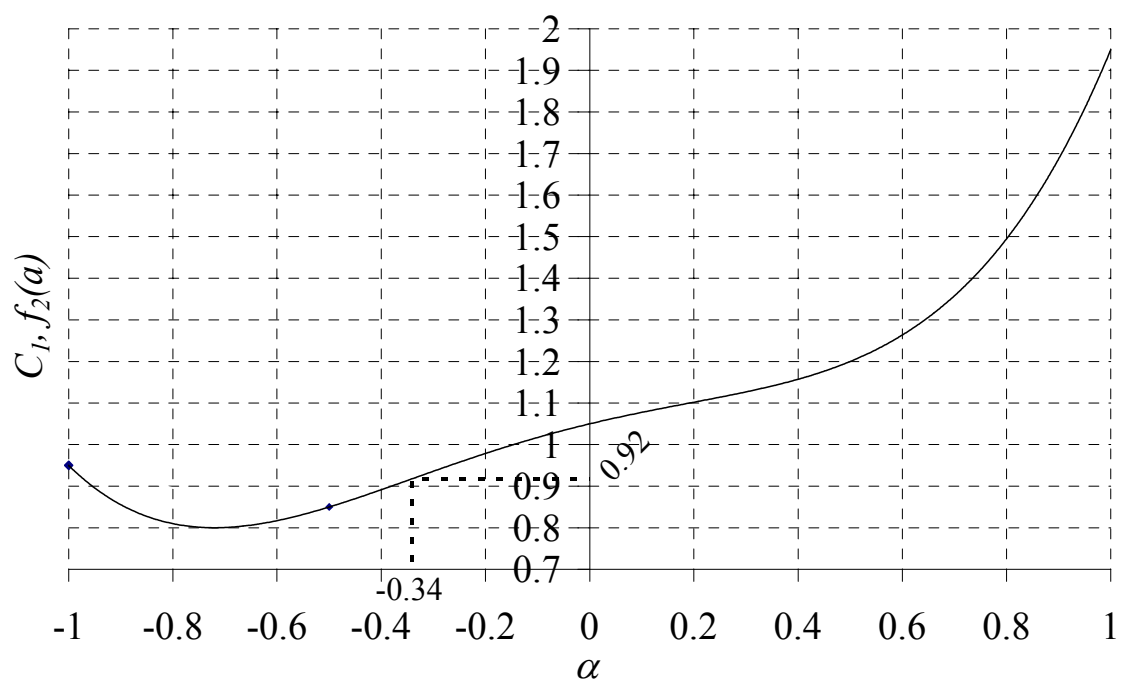

Figure 27. $c_{l}$ with Respect to $\quad \alpha$ for G3 Profiled Steel Sheeting 


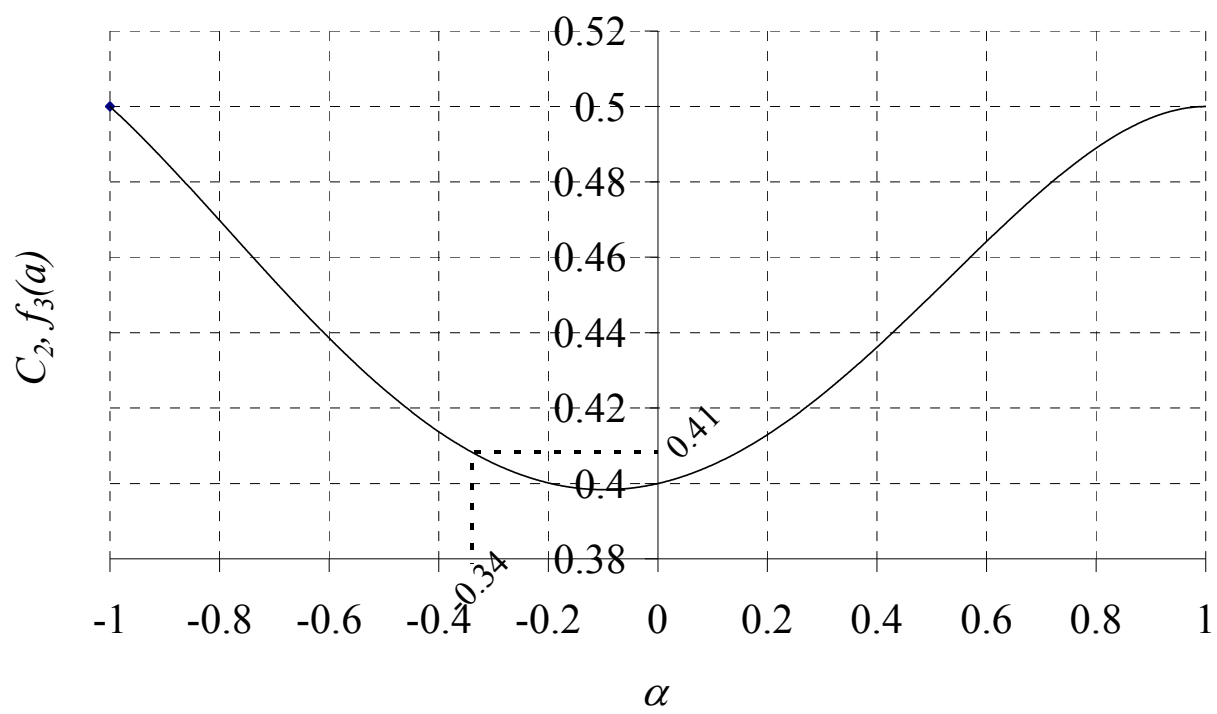

Figure 28. $c_{2}$ with Respect to $\alpha$ for G3 Profiled Steel Sheeting

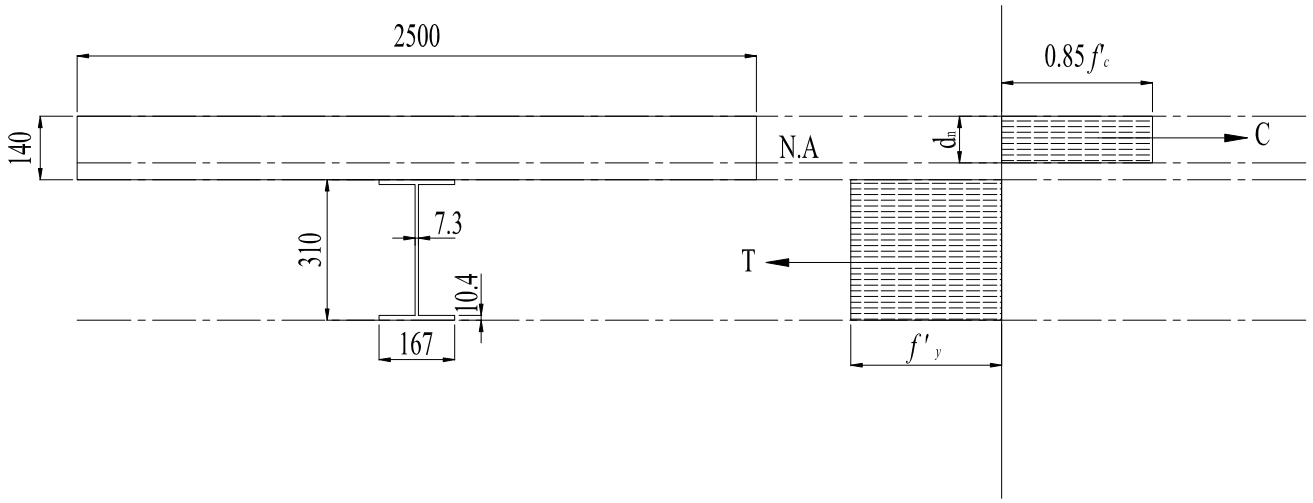

Figure 29. Beam Cross Section for G3 Profiled Steel Sheeting

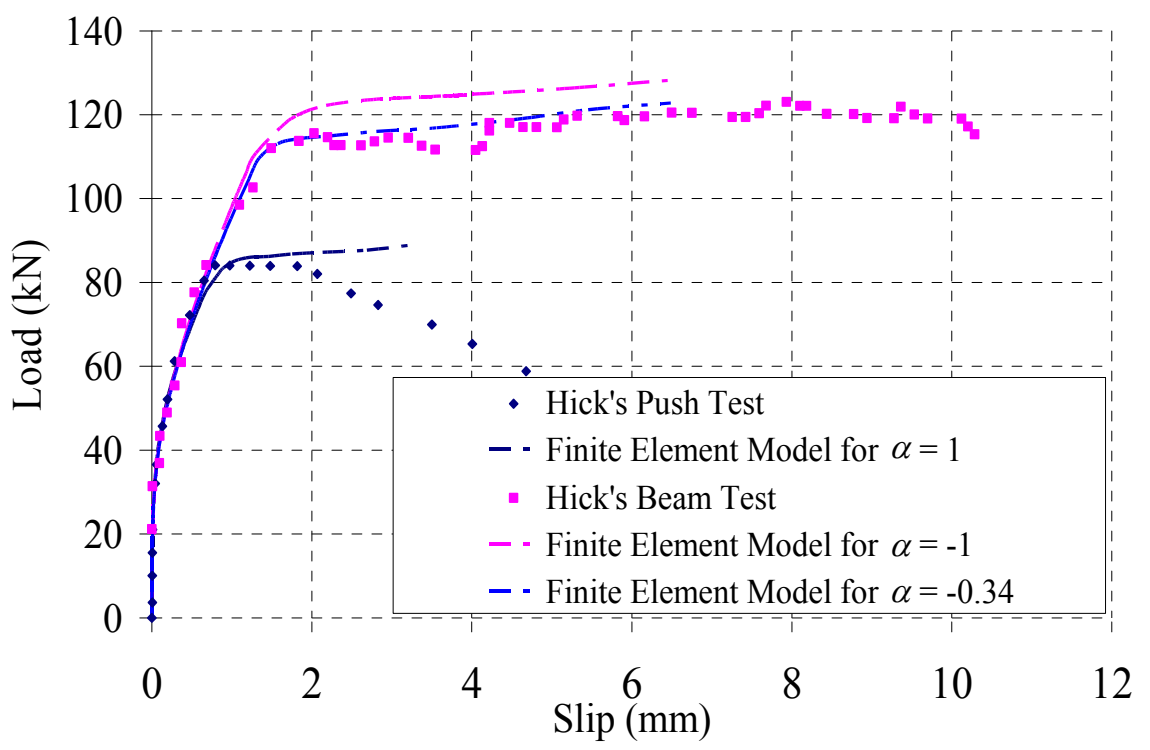

Figure 30. Comparison between Experimental Data and Finite Element Model for Hick's Trapezoidal Profiled Steel Sheeting 


\subsection{Profiled Slabs Compared with Available International Standards}

A comparison was made to evaluate the finite element solutions from G1, G2 and G3 with three existing international standards. They included the Australian Standard AS 2327 [16], British Standards Institute [17] and American Standard AISC [18].

Two failure modes can be observed to investigate the ultimate shear capacity of the shear connector in the push tests. They include shear failure and concrete failure. When the concrete strength is relatively high, the fracture of studs will often govern the design, and it is given by Eq. 5a, b and c.

$$
\begin{aligned}
& P_{A S}=0.63 d^{2} f_{u s} \\
& P_{E C}=0.628 d^{2} f_{u s} \\
& P_{A I S C}=0.785 d^{2} f_{u s}
\end{aligned}
$$

where

$E_{s c}$ is the modulus of elasticity of the shear connector,

$f_{c}$ is the compressive strength of the concrete,

$f_{u s}$ is the ultimate strength of the shear connector.

However, when the shear connector is stronger than concrete strength, Eq. 6a, b and c will tend to govern.

$$
\begin{aligned}
& P_{A S}=0.31 d^{2} \sqrt{f_{c k} E_{c}} \\
& P_{E C}=0.29 d^{2} \sqrt{f_{c k} E_{c}} \\
& P_{A I S C}=0.39 d^{2} \sqrt{f_{c k} E_{c}}
\end{aligned}
$$

where

$f_{c k}$ is the characteristic compressive cylinder strength of the concrete

$E_{c}$ is defined as the mean modulus of elasticity of the concrete.

When the steel profiled slab is taken into consideration, equations from (5 and 6) above are multiplied by a reduction factor $k$. The value of $k$ is defined in the equations below:

$$
\begin{aligned}
& k_{A S}=1.148-(0.18 / \sqrt{n}) \\
& k_{E C}=\left(\frac{0.7}{\sqrt{n}}\right)\left(\frac{b_{o}}{h_{p}}\right)\left(\left(\frac{h}{h_{p}}\right)-1\right) \leq k_{t, \text { lim }} \\
& k_{A I S C}=\left(\frac{0.85}{\sqrt{n}}\right)\left(\frac{b_{o}}{h_{p}}\right)\left(\left(\frac{h}{h_{p}}\right)-1\right) \leq 1
\end{aligned}
$$

where

$n$ is the number of studs

$b_{o}$ is the width of the steel profiled rib

$h_{p}$ is the height of the decking profile

$h$ is the height of the stud 
Table 4. Comparison of Ultimate Load for Shear Connector between Finite Element Models and International Standards

\begin{tabular}{|c|c|c|c|c|c|c|c|c|c|c|}
\hline \multicolumn{11}{|c|}{ Concrete Property, $\mathrm{f}_{\mathrm{c}}=20 \mathrm{MPa}$} \\
\hline \multirow{3}{*}{$\begin{array}{l}\text { Profiled } \\
\text { Type }\end{array}$} & \multirow[b]{2}{*}{ FEM Result } & \multicolumn{2}{|c|}{$\begin{array}{l}\text { Australian } \\
\text { Standard }\end{array}$} & \multicolumn{2}{|c|}{ Eurocode } & \multicolumn{2}{|c|}{$\begin{array}{c}\text { American } \\
\text { Standard }\end{array}$} & \multicolumn{3}{|c|}{ FEM/Standard } \\
\hline & & SF & $\mathrm{CF}$ & $\mathrm{SF}$ & $\mathrm{CF}$ & SF & $\mathrm{CF}$ & \multirow[b]{2}{*}{ AS } & \multirow[b]{2}{*}{$\mathrm{EC}$} & \multirow[b]{2}{*}{ AISC } \\
\hline & \multicolumn{7}{|c|}{$(\mathrm{kN})$} & & & \\
\hline $\mathrm{G} 1$ & $73.1(\mathrm{CF})$ & 116.1 & 74.6 & 80.5 & 48.5 & 122.1 & 79.3 & 0.98 & 1.51 & 0.92 \\
\hline G2 & $70.3(\mathrm{CF})$ & 116.1 & 74.6 & 113.5 & 68.5 & 141.7 & 111.8 & 0.94 & 1.03 & 0.63 \\
\hline G3 & $68.8(\mathrm{CF})$ & 116.1 & 74.6 & 85.2 & 51.4 & 129.3 & 83.9 & 0.92 & 1.34 & 0.82 \\
\hline \multirow{4}{*}{$\begin{array}{c}\text { Profiled } \\
\text { Type }\end{array}$} & \multirow{2}{*}{\multicolumn{3}{|c|}{$\begin{array}{c}\text { Concrete } \\
\text { Australian } \\
\text { Standard }\end{array}$}} & Proper & ies, f' & $=25 \mathrm{~N}$ & $\mathbf{P a}$ & & & \\
\hline & & & & \multicolumn{2}{|c|}{ Eurocode } & \multicolumn{2}{|c|}{$\begin{array}{c}\text { American } \\
\text { Standard }\end{array}$} & \multicolumn{3}{|c|}{ FEM/Standard } \\
\hline & FEM Result & SF & CF & SF & $\mathrm{CF}$ & SF & $\mathrm{CF}$ & \multirow[b]{2}{*}{ AS } & & \multirow[b]{2}{*}{ AISC } \\
\hline & \multicolumn{7}{|c|}{$(\mathrm{kN})$} & & $\mathrm{EC}$ & \\
\hline G1 & $81.5(\mathrm{CF})$ & 116.1 & 89.6 & 80.5 & 58.3 & 122.1 & 95.2 & 0.70 & 1.01 & 0.67 \\
\hline G2 & $78.2(\mathrm{CF})$ & 116.1 & 89.6 & 113.5 & 82.2 & 141.7 & 110.4 & 0.67 & 0.69 & 0.55 \\
\hline G3 & $76.2(\mathrm{CF})$ & 116.1 & 89.6 & 85.2 & 61.7 & 129.3 & 100.8 & 0.66 & 0.89 & 0.59 \\
\hline \multirow{4}{*}{$\begin{array}{l}\text { Profiled } \\
\text { Type }\end{array}$} & \multirow{2}{*}{\multicolumn{3}{|c|}{$\begin{array}{c}\text { Concrete } \\
\text { Australian } \\
\text { Standard }\end{array}$}} & Proper & ies, f' & $=32 \mathrm{~N}$ & & & & \\
\hline & & & & \multicolumn{2}{|c|}{ Eurocode } & \multicolumn{2}{|c|}{$\begin{array}{c}\text { American } \\
\text { Standard }\end{array}$} & \multicolumn{3}{|c|}{ FEM/Standard } \\
\hline & FEM Result & SF & $\mathrm{CF}$ & $\mathrm{SF}$ & $\mathrm{CF}$ & SF & $\mathrm{CF}$ & \multirow[b]{2}{*}{ AS } & & \\
\hline & \multicolumn{7}{|c|}{$(\mathrm{kN})$} & & $\mathrm{EC}$ & AISC \\
\hline G1 & $93.2(\mathrm{CF})$ & 116.1 & 113.3 & 80.5 & 73.7 & 122.1 & 120.4 & 0.80 & 1.16 & 0.76 \\
\hline G2 & $89.3(\mathrm{CF})$ & 116.1 & 113.3 & 113.5 & 104.1 & 141.7 & 139.7 & 0.77 & 0.79 & 0.63 \\
\hline G3 & $86.6(\mathrm{CF})$ & 116.1 & 113.3 & 85.2 & 78.1 & 129.3 & 127.5 & 0.75 & 1.02 & 0.67 \\
\hline & & Co & ccrete & Proper & ies, $\mathbf{f}^{\prime}$ & $=35 \mathrm{~N}$ & $\mathrm{~Pa}$ & & & \\
\hline & & $\begin{array}{l}\text { Aust } \\
\text { Stan }\end{array}$ & $\begin{array}{l}\text { ralian } \\
\text { dard }\end{array}$ & Euro & code & $\begin{array}{l}\text { Ame } \\
\text { Stan }\end{array}$ & $\begin{array}{l}\text { ican } \\
\text { dard }\end{array}$ & & M/Star & ard \\
\hline Profiled & FEM Result & $\mathrm{SF}$ & $\mathrm{CF}$ & SF & $\mathrm{CF}$ & SF & $\mathrm{CF}$ & & & \\
\hline Type & & & & N) & & & & AS & $\mathrm{EC}$ & AISC \\
\hline G1 & $98.2(\mathrm{CF})$ & 116.1 & 110.7 & 80.5 & 72 & 122.1 & 117.6 & 0.85 & 1.22 & 0.80 \\
\hline G2 & $94.1(\mathrm{CF})$ & 116.1 & 110.7 & 113.5 & 101.6 & 141.7 & 136.4 & 0.81 & 0.83 & 0.66 \\
\hline G3 & $91.1(\mathrm{CF})$ & 116.1 & 110.7 & 85.2 & 76.2 & 129.3 & 124.5 & 0.78 & 1.07 & 0.70 \\
\hline & & Co & ncrete & $10 p$ & es, $f^{\prime}$ & $=40 \mathrm{~N}$ & & & & \\
\hline & & $\begin{array}{r}\text { Aust } \\
\text { Stan }\end{array}$ & $\begin{array}{l}\text { ralian } \\
\text { dard }\end{array}$ & Euro & code & $\begin{array}{l}\text { Ame } \\
\text { Stan }\end{array}$ & $\begin{array}{l}\text { ican } \\
\text { dard }\end{array}$ & & M/Star & ard \\
\hline Profiled & FEM Result & $\mathrm{SF}$ & $\mathrm{CF}$ & SF & $\mathrm{CF}$ & SF & $\mathrm{CF}$ & & & \\
\hline Type & & & & N) & & & & $\mathrm{AS}$ & $\mathrm{EC}$ & AISC \\
\hline G1 & 103.7 (SF) & 116.1 & 126.7 & 80.4 & 82.4 & 122.1 & 134.6 & 0.89 & 1.29 & 0.85 \\
\hline G2 & $100.4(\mathrm{SF})$ & 116.1 & 126.7 & 113.5 & 116.3 & 141.7 & 156.2 & 0.86 & 0.88 & 0.71 \\
\hline G3 & $97.4(\mathrm{SF})$ & 116.1 & 126.7 & 85.1 & 87.3 & 129.3 & 142.5 & 0.84 & 1.14 & 0.75 \\
\hline
\end{tabular}




\begin{tabular}{|c|c|c|c|c|c|c|c|c|c|c|}
\hline \multicolumn{11}{|c|}{ Concrete Properties, $\mathrm{f}_{\mathrm{c}}=50 \mathrm{MPa}$} \\
\hline \multirow{3}{*}{$\begin{array}{c}\text { Profiled } \\
\text { Type }\end{array}$} & \multirow[b]{2}{*}{ FEM Result } & \multicolumn{2}{|c|}{$\begin{array}{l}\text { Australian } \\
\text { Standard }\end{array}$} & \multicolumn{2}{|c|}{ Eurocode } & \multicolumn{2}{|c|}{$\begin{array}{c}\text { American } \\
\text { Standard }\end{array}$} & \multicolumn{3}{|c|}{ FEM/Standard } \\
\hline & & SF & $\mathrm{CF}$ & SF & $\mathrm{CF}$ & SF & $\mathrm{CF}$ & & & \\
\hline & \multicolumn{7}{|c|}{$(\mathrm{kN})$} & AS & $\mathrm{EC}$ & AISC \\
\hline $\mathrm{G1}$ & $114.7(\mathrm{SF})$ & 116.1 & 141.7 & 80.4 & 92.2 & 122.1 & 150.5 & 0.99 & 1.43 & 0.94 \\
\hline G2 & $112.9(\mathrm{SF})$ & 116.1 & 141.7 & 113.5 & 130.1 & 141.7 & 174.6 & 0.97 & 0.99 & 0.80 \\
\hline G3 & $110.1(\mathrm{SF})$ & 116.1 & 141.7 & 85.1 & 97.6 & 129.3 & 159.4 & 0.95 & 1.29 & 0.85 \\
\hline
\end{tabular}

* S.F denotes stud failure and C.F concrete failure and

* Shaded area denotes failure mode govern

A summary of the ultimate loads for the shear connector, $P$ which are calculated using the Australian Standard, Eurocode and American Standard is given in Table 4. Moreover, authors analysed the finite element model using different concrete strength which includes $f_{c}{ }_{c}=20 \mathrm{MPa}$, $25 \mathrm{MPa}, 32 \mathrm{MPa}, 35 \mathrm{MPa}, 40 \mathrm{MPa}$ and $50 \mathrm{MPa}$. Table 4 verified that when the concrete strength is lower the concrete failure will governed whilst when the concrete strength is higher, the stud fracture failure tends to govern.

For concrete strength with $f^{\prime}{ }_{c}=20 \mathrm{MPa}$ to $35 \mathrm{MPa}$, the finite element model demonstrated that the concrete stress reached maximum value before the stress in shear studs. Therefore, this verified that the push tests failed due to concrete failure. The failure modes predicted by existing standards also similar to the finite element models. From Table 4, the finite element model showed a more conservative value when compared to Australian and American Standard. On the other hand, Eurocode seems to be overly conservative.

When the concrete strength increased, the finite element model shows that the shear stud reaches its maximum stress before concrete slab. This proved that the failure mode is governed by stud fractured. When compared with the existing standards, the calculated theoretical values for both the Australian and American standards seem to have higher values when compared with finite element models. This confirmed that the finite element model is more conservative than the two existing standards. On the contrary, the Eurocode seems to be very conservative, where the calculated theoretical value is lower than the finite element predicted value.

\section{CONCLUSIONS}

Shear connection nonlinearity always results in significant changes in the strength and ductility of composite steel-concrete beams. In order to highlight such phenomena, a non-linear procedure with different strain regimes implemented in the concrete element has been presented to account for both the shear connection strength and ductility.

A comparison with experimental results allows the validation of such a procedure with reference to both solid slabs and profiled slabs for both push tests and beam tests. The finite element analysis that has been undertaken in this paper demonstrated that the push test to determine the shear connection capacity for solid slabs is reliable and accurate. However this is not the case for slabs with profiled steel sheeting.

An extensive parametric study of fifteen push test specimens with different profiled steel sheeting geometries was performed using the established finite element models. The comparison of shear connector capacities obtained from the finite element models proved that they depend significantly on the width and rib types of profiled steel sheeting. 
The introduction of the parameters such as $\alpha, Q_{U}, c_{1}$ and $c_{2}$ are the key characteristics of the proposed method because they account for the behaviour of the shear connection nonlinearity effects. When accurate parameter values were applied to the force-slip relationship equations, it seems to suggest that the proposed method has less discrepancy when compared with the experimental study, and it is also useful for design engineers using these profiles in industry.

When the Australian, Eurocode and American Standards are compared with finite element results, the Australian Standards and American Standards appeared to be overestimating the shear connector capacity whilst Eurocode grossly underestimated the strength of shear connectors for profiled slabs. From the three existing standards, the American Standard seems to be less conservative where higher ultimate load was observed.

This study also provides a very initial study of this issue. Further experimental studies and more substantial parametric studies would be necessary before being able to employ these results in international code recommendations.

\section{ACKNOWLEDGEMENTS}

The authors would like to thank the Australian Research Council and BlueScope Lysaght Sydney for providing funding for this project and the University of Western Sydney for providing a conducive environment for the authors to prepare this paper.

\section{REFERENCES}

[1] British Standards Institution, "Design of Composite Steel and Concrete Structures, Part 1.1 General Rules and Rules for Buildings, British Standard Institute, London", 2004.

[2] Hicks, S.J., "Resistance and Ductility of Shear Connection: Full-scale Beam and Push Tests", 6th International Conference on Steel \& Aluminium Structures, St. Catherine College, University of Oxford, England, 2007, pp. 613-620

[3] Ranzi, G., Bradford, M.A. and Uy, B., "A Direct Stiffness Analysis of a Composite Beam with Partial Interaction", International Journal for Numerical Methods in Engineering, 2004, Vol. 61, No. 5, pp. 657-672.

[4] Popov, E.P. and Balan, T.A., "Engineering Mechanics of Solids", Prentice Hall, Upper Saddle river, New Jersey, 1998.

[5] Carreira, D. and Chu, K., "Stress-strain Relationship for Plain Concrete in Compression", Journal of ACI Structural, 1985, Vol. 82, No. 11, pp. 797-804.

[6] Loh, H.Y., Uy, B. and Bradford, M.A., "The Effects of Partial Shear Connection in the Hogging Moment Region of Composite Beams Part II - Analytical Study", Journal of Constructional Steel Research, 2003, Vol. 60, pp. 921-962.

[7] Lam, D. and El-Lobody, E., "Behaviour of Headed Stud Shear Connectors in Composite Beam", Journal of Structural Engineering-ASCE, 2005, Vol. 131, No.1, pp. 96-107.

[8] Mirza, O. and Uy, B., "Effect of Steel Fibre Reinforcement on the Shear Connection of Composite Steel-Concrete Beams", Journal of Advanced Steel Construction, 2007, In press.

[9] Johnson, R.P. and Yuan, H., "Existing Rules and New Tests for Stud Shear Connectors in troughs of Profiled Sheeting", Proceedings of the Institution of Civil Engineers, Structures and Buildings, 1998a, Vol. 128, No.3, pp. 244-251.

[10] Johnson, R.P. and Yuan, H., "Models and Design Rules for Stud Shear Connectors in troughs of Profiled Sheeting", Proceedings of the Institution of Civil Engineers, Structures and Buildings, 1998b, Vol. 128, No.3, pp. 252-265. 
[11] Karlsson and Sonrensen, "ABAQUS, Theory manual version 6.5", Hibbitt Publication, Pawtucket, Rhode Island, 2006a.

[12] Karlsson and Sonrensen, "ABAQUS User's manual version 6.5", Hibbitt Publication Pawtucket, Rhode Island, 2006b.

[13] Karlsson and Sonrensen, "ABAQUS Analysis user's manual version 6.5", Hibbitt Publication Pawtucket, Rhode Island, 2006c.

[14] El-Lobody, E. and Young, B., "Performance of Shear Connection in Composite Beams with Profiled Steel Sheeting", Journal of Constructional Steel Research, 2006, Vol. 62, No.7, pp. 682-694.

[15] Aribert, J. and Labib, A., "Modèle Calcul èlasto-plastique de Pustres Mixtes a Connexion Partielle", Journal of Construct Metallique, 1982, Vol. 4, pp. 3-51.

[16] AS 2327, A. S., "Composite structures, Part 1: Simply supported beams", Standard Australia International, 2003, AS 2327.1-2003.

[17] British Standards Institute, "Design of composite steel and concrete structures, Part 1.1 General rules and rules for buildings, British Standard Institute, London", 2004.

[18] AISC, "Load and resistance factor design specification (LRFD)", American Institute of Steel Construction, 1999. 


\title{
AN INVESTIGATION ON THE POST-LOCAL-BUCKLING ANALYSIS OF I-SECTION STRUTS USING FINITE STRIP METHOD
}

\author{
H.R. Ovesy ${ }^{1, *}$, J. Loughlan ${ }^{2}$ and S.A.M. Ghannadpour ${ }^{3}$ \\ ${ }^{1}$ Aerospace Engineering Department and Centre of Excellence in Computational Aerospace Engineering, \\ Amirkabir University of Technology, Tehran, Iran \\ ${ }^{2}$ Department of Aeronautical and Automotive Engineering, Loughborough University, \\ Leicestershire LE11 3TU, UK \\ ${ }^{3}$ Space Engineering Dept., Faculty of New Technologies and Energy Engineering, Shahid Beheshti \\ University, G.C., Evin, 1983963113, Tehran, Iran \\ *(Corresponding author: E-mail: ovesy@aut.ac.ir)
}

Received: 29 August 2008; Revised: 22 November 2008; Accepted: 26 November 2008

\begin{abstract}
Two finite strip methods are developed for predicting the geometrically non-linear response of I-sections with simply supported ends when subjected to uniform end shortening in their plane. Although the formulations of both finite strip methods are based on the concept of the principle of minimum potential energy, the first finite strip method utilizes a semi-energy finite strip, whereas in the second finite strip method (which is designated by the name full-energy finite strip method) all the displacements are postulated by the appropriate shape functions. Both finite strip methods are then applied to analyze the post-local-buckling behavior of some representative I-section struts.
\end{abstract}

Keywords: Geometric Non-linear; Post-Local-Buckling; Semi-Energy; Finite Strip; I- Section

\section{INTRODUCTION}

Prismatic plates and plate structures are often employed in situations where they are subjected to in-plane compressive loading. Thus it is important to accurately predict the buckling and post buckling behavior of such structures. The post-local-buckling behavior of elastic plates or plate structures is a geometric non-linear problem. Among the energy-based approximate methods for the case of prismatic structures, the finite strip method (FSM) [1], which is a special form of the finite element method, has proved to be a capable tool for analyzing the post-buckling behavior of plates and plate structures. A typical example of a strut that is modeled by finite strips is shown in Figure 1.
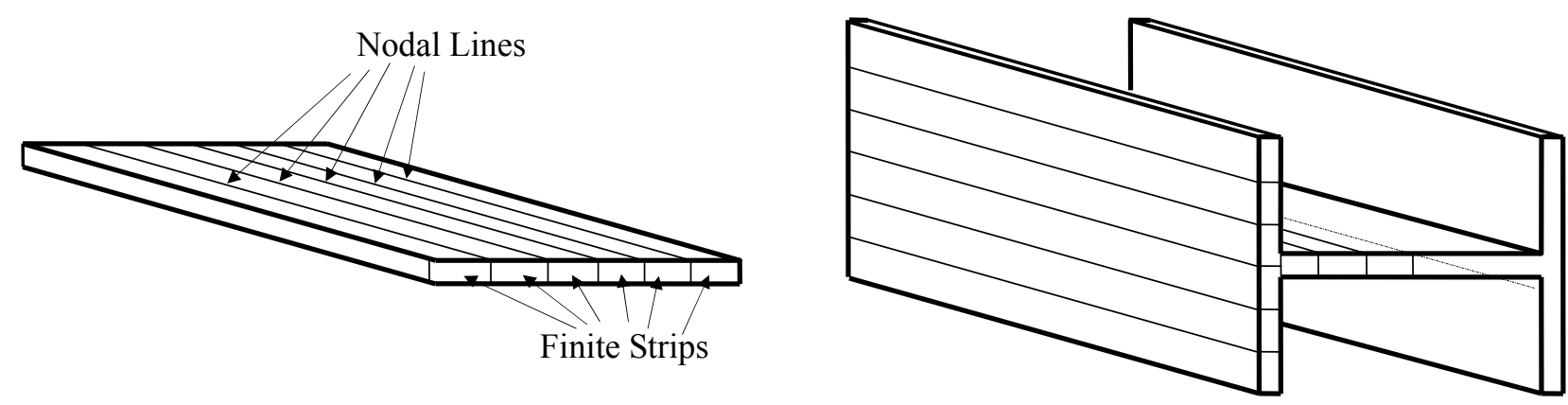

Figure 1. Plate Structures Discretized by Finite Strips 
Early works concerned with the use of the FSM in predicting the geometrically non-linear response of single rectangular plates and prismatic plate structures are those of Graves Smith and Sridharan [1, $2 \& 3]$ and Hancock [4]. These authors consider the post-buckling behaviour of plate structures subjected to uniform [ $1 \& 3]$ or linearly varying $[2,4]$ end shortening, with each component plate of the structure having simply supported ends. The elastic post-buckling response of channel section struts [1] and rectangular box columns [3] are investigated by Graves Smith and Sridharan. Hancock uses the finite strip method to investigate the post-buckling behaviour of square box and I-section columns. In the finite strip methods developed by the aforementioned authors, in-plane displacement fields are postulated in addition to the out-of-plane displacement field. Hence, these methods are to be designated as the full-energy FSM in the current paper. Since the time of the early publications discussed above, there has been a significant amount of work published on the application of the FSM to post-local-buckling behaviour of plates and plate structures. Dawe et al. [5] have developed a semi-analytical FSM for analyzing the post-local-buckling problem of composite plate structures. The finite strip properties are based on the use of both classical plate theory and first-order shear deformation plate theory, and the method is applied to analyze the post-local-buckling behaviour of box sections and top-hat-stiffened and blade-stiffened panels subjected to progressive uniform end shortening. Kwon and Hancock [6] have developed a non-linear elastic spline FSM to study the post-buckling behaviour of isotropic thin-walled sections undergoing local and distortional buckling. Azhari and Bradford [7] have developed a non-linear semi-analytical FSM for analyzing the elastic post-local-buckling behaviour of geometrically imperfect plate assemblies. The plate assemblies are assumed to be made of isotropic materials. In this method, the finite strip displacement functions, used earlier by Graves Smith and Sridharan [1], are augmented with so-called bubble functions, which are extra modes associated with internal or nodeless degrees of freedom. The method is deployed to study the post-local-buckling behaviour of channels under various compression eccentricities, and I-section members under pure compression and pure bending. An energy-based approximate method, referred to as the semi-energy method by Rhodes \& Harvey [8], was first used by Marguerre [9] in 1937, and has since been used by various researchers. More recently, Ovesy et al. [10-12] have developed a semi-energy post-local-buckling FSM in which the out-of-plane displacement of the finite strip is the only displacement which is postulated by a deflected form as opposed to that mentioned previously with respect to the full-energy FSM. The developed semi-energy FSM has been applied to analyze the post-local-buckling behavior of thin flat plates [10], open channel section [11] and box section struts [12]. Although a considerable amount of work has been published on the post-local-buckling behavior of plates and plate structures by using different versions of FSM, no work has been reported in which a thorough comparison is made between the semi-energy FSM and the more conventional full-energy FSMs, when applied to the post local- buckling analysis of I-sections. This task is fulfilled in this paper by developing a semi-energy post-local-buckling finite strip method as well as a full-energy finite strip method based on the theory proposed by Dawe et al. [5]. The developed finite strip methods are then applied to analyze the post-local-buckling behavior of some representative I-sections.

\section{THEORETICAL DEVELOPMENTS}

In this section, the fundamental elements of the theory for the developed finite strip methods are briefly outlined. It is noted that a perfectly flat finite strip made up of a linear isotropic material (with a constant modulus of elasticity $\mathrm{E}$ and Poisson ratio $v$ ) is assumed throughout the theoretical developments of this paper. The finite strip, which is schematically shown in Figure 2, is of thickness t. It may be noted that $u, v$ and $w$ correspond to the mid-plane displacements, and the finite strip is assumed to be simply supported out-of-plane at the loaded ends (i.e. at ends $x=0 \& \mathrm{~L}$ ). Moreover, the finite strip is assumed to be subjected to an in-plane compressive loading acting 
through frictionless rigid platens such that uniform end shortening $\bar{u}$ occurs at end $x=\mathrm{L}$ only (see Figure 2). The boundary conditions at loaded ends of the finite strip are summarized as follows:

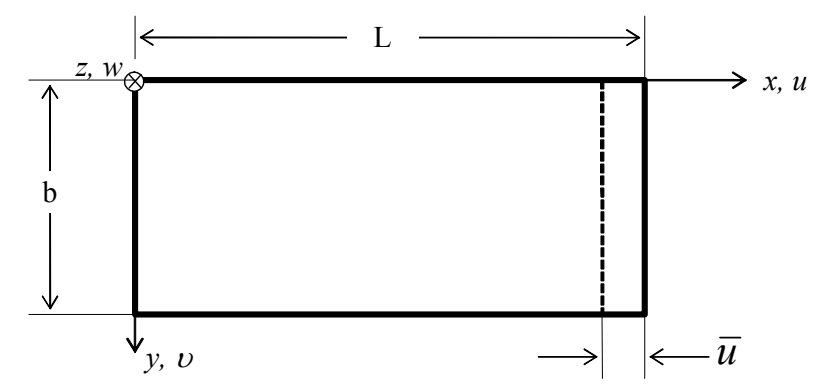

Figure 2. A Finite Strip Subjected to a Prescribed Uniform End Shortening

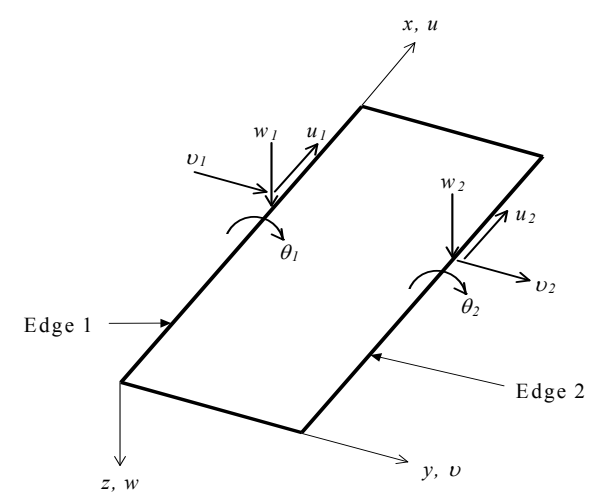

Figure 3. Displacement Parameters of a Finite Strip

$$
w=M_{x}=N_{x y}=0 \quad \text { at } \quad x=0 \& L \text { and } u= \begin{cases}0 & \text { at } \quad x=0 \\ -\bar{u} & \text { at } \quad x=L\end{cases}
$$

It is emphasized that the Classical Plate Theory (CPT) is applied in the remaining of the paper. As a result of this assumption, the Kirchhoff normalcy condition is incorporated, and thus:

$$
\begin{aligned}
& u_{t}(x, y, z)=u(x, y)-z \frac{\partial w(x, y)}{\partial x} \\
& v_{t}(x, y, z)=v(x, y)-z \frac{\partial w(x, y)}{\partial y} \\
& w_{t}(x, y, z)=w(x, y)
\end{aligned}
$$

where $u_{t}, v_{t}$ and $w_{t}$ are components of displacement at a general point, whilst $u, v$ and $w$ are similar components at the middle surfaces $(z=0)$.

On the assumption that the plate is in a state of plane stress, the stress-strain relationship at a general point for the plate becomes:

$$
\overline{\boldsymbol{\sigma}}=\left\{\begin{array}{l}
\bar{\sigma}_{x} \\
\bar{\sigma}_{y} \\
\bar{\tau}_{x y}
\end{array}\right\}=\frac{E}{1-v^{2}}\left[\begin{array}{ccc}
1 & v & 0 \\
v & 1 & 0 \\
0 & 0 & \frac{1-v}{2}
\end{array}\right] . \bar{\varepsilon} ; \bar{\varepsilon}=\left\{\begin{array}{l}
\bar{\varepsilon}_{x} \\
\bar{\varepsilon}_{y} \\
\bar{\gamma}_{x y}
\end{array}\right\}
$$

Where $\bar{\sigma}$ and $\overline{\boldsymbol{\varepsilon}}$, respectively, correspond to the stresses and strains at a general point. It is noted that these stresses and strains include the components corresponding to the membrane and bending contributions as outlined below :

$$
\bar{\sigma}=\sigma+\sigma_{b} ; \bar{\varepsilon}=\varepsilon+\varepsilon_{b}
$$


It is also noted that the relationship between $\sigma$ and $\varepsilon$, which correspond to the membrane contribution, is similar to that given by Eq. 3. The same relationship also applies between $\sigma_{b}$ and $\boldsymbol{\varepsilon}_{b}$, which correspond to the bending contribution. Moreover, the membrane strain $\varepsilon$ can be subdivided into its linear $\boldsymbol{\varepsilon}_{\boldsymbol{l}}$ and non-linear $\boldsymbol{\varepsilon}_{\boldsymbol{n}}$ components as given below:

$$
\boldsymbol{\varepsilon}=\boldsymbol{\varepsilon}_{l}+\boldsymbol{\varepsilon}_{n l}=\left\{\begin{array}{c}
\frac{\partial u}{\partial x} \\
\frac{\partial v}{\partial y} \\
\frac{\partial u}{\partial y}+\frac{\partial v}{\partial x}
\end{array}\right\}+\left\{\begin{array}{c}
\frac{1}{2}\left(\frac{\partial w}{\partial x}\right)^{2} \\
\frac{1}{2}\left(\frac{\partial w}{\partial y}\right)^{2} \\
\frac{\partial w}{\partial x} \frac{\partial w}{\partial y}
\end{array}\right\}
$$

It is also noted that within the context of CPT the bending strains $\boldsymbol{\varepsilon}_{b}$ are expressed by the following equations:

$$
\boldsymbol{\varepsilon}_{\boldsymbol{b}}=\left\{\begin{array}{c}
-z \frac{\partial^{2} w}{\partial x^{2}} \\
-z \frac{\partial^{2} w}{\partial y^{2}} \\
-2 z \frac{\partial^{2} w}{\partial x \partial y}
\end{array}\right\}
$$

Since the potential energy of external loads is zero for the strip under consideration, the total potential energy of the strip $V_{s}$ is simply equal to the its strain energy $U_{s}$ (i.e. $V_{s}=U_{s}$ ) which is given below:

$$
U_{s}=\frac{1}{2} \iiint\left(\bar{\sigma}_{x} \bar{\varepsilon}_{x}+\bar{\sigma}_{y} \bar{\varepsilon}_{y}+\bar{\tau}_{x y} \bar{\gamma}_{x y}\right) d x d y d z
$$

By substituting $\bar{\sigma}$ and $\bar{\varepsilon}$ from Eq. 4 into Eq. 7 and rearranging, the total strain energy of the strip $U_{s}$ can be expressed by the following Equations:

$$
U_{s}=U_{m s}+U_{b s}
$$

Where $U_{m s}$ designates the membrane strain energy of the strip and is given by:

$$
U_{m s}=\frac{1}{2} \iiint\left(\sigma_{x} \varepsilon_{x}+\sigma_{y} \varepsilon_{y}+\tau_{x y} \gamma_{x y}\right) d x d y d z
$$

and $U_{b s}$ designates the bending strain energy of the strip and is given by: 


$$
U_{b s}=\frac{1}{2} \iiint\left(\sigma_{b x} \varepsilon_{b x}+\sigma_{b y} \varepsilon_{b y}+\tau_{b x y} \gamma_{b x y}\right) d x d y d z
$$

The membrane strain energy $U_{m s}$ is further expanded by substituting for $\boldsymbol{\sigma}$ in terms of $\boldsymbol{\varepsilon}$ using Eq. 3 , and subsequently carrying out the integration in the $z$ direction. This gives:

$$
U_{m s}=\frac{\mathrm{A}}{2} \iint\left(\varepsilon_{x}^{2}+\varepsilon_{y}^{2}+2 v \varepsilon_{x} \varepsilon_{y}+\frac{1-v}{2} \gamma_{x y}^{2}\right) d x d y
$$

Where $\mathrm{A}=\mathrm{Et}_{\mathrm{s}} /\left(1-v^{2}\right)$ is the so-called in-plane stiffness of the strip.

The bending strain energy $U_{b s}$ is also expanded by using Eq. 3, and subsequently substituting for $\boldsymbol{\varepsilon}_{b}$ by implementing Eq. 6, and finally carrying out the integration in the $z$ direction. This gives:

$$
U_{b s}=\frac{D^{2}}{2} \int_{0}^{b_{s}} \int_{0}^{L}\left\{\left(\frac{\partial^{2} w}{\partial x^{2}}+\frac{\partial^{2} w}{\partial y^{2}}\right)^{2}+2(1-v)\left[\left(\frac{\partial^{2} w}{\partial x \partial y}\right)^{2}-\frac{\partial^{2} w}{\partial x^{2}} \frac{\partial^{2} w}{\partial y^{2}}\right]\right\} d x d y
$$

Where $\mathrm{D}=\mathrm{E} \mathrm{t}^{3} / 12\left(1-v^{2}\right)$ is the so-called bending stiffness of the strip.

\subsection{Theoretical Developments of Semi-energy Finite Strip}

A more detailed coverage of the theoretical aspects and developments of the semi-energy finite strip approach are given in an earlier publication by Ovesy et al [10], which pertains to the compressive post-buckling behaviour of thin plates, and the reader is referred to this reference for a deeper insight into the method. In the present paper, however, the authors feel that in the application of the approach to structural sections a theoretical presentation of the method is, indeed, necessary but that this should only include the dominating features of the method and thus these are detailed in the paper in a reduced outline presentation of the approach.

As mentioned earlier, in the semi-energy method, the out-of-plane displacement of the finite strip is the only displacement which is postulated by a deflected form. Then, the postulated deflected form is substituted into von Kármán's compatibility equation which is solved exactly to obtain the form of the in-plane displacements that correspond to the postulated form of the out-of-plane displacement. The von Kármán's compatibility equation for large deflections of plate is given by Eq. 13.

$$
\nabla^{4} \mathrm{~F}=\frac{\partial^{4} \mathrm{~F}}{\partial x^{4}}+2 \frac{\partial^{4} \mathrm{~F}}{\partial x^{2} \partial y^{2}}+\frac{\partial^{4} \mathrm{~F}}{\partial y^{4}}=\mathrm{E}\left\{\left(\frac{\partial^{2} w}{\partial x \partial y}\right)^{2}-\left(\frac{\partial^{2} w}{\partial x^{2}}\right)\left(\frac{\partial^{2} w}{\partial y^{2}}\right)\right\}
$$

In this equation the function $\mathrm{F}$ (i.e. $\mathrm{F}=\mathrm{F}(x, y)$ ) which is known as the Airy stress function is defined as follows: 


$$
\begin{aligned}
& \sigma_{x}=\frac{\partial^{2} \mathrm{~F}(x, y)}{\partial y^{2}} \\
& \sigma_{y}=\frac{\partial^{2} \mathrm{~F}(x, y)}{\partial x^{2}} \\
& \tau_{x y}=-\frac{\partial^{2} \mathrm{~F}(x, y)}{\partial x \partial y}
\end{aligned}
$$

It is noted that the function given by Eq. 15 has been found to be suitable for the representation of the out-of-plane displacement field for the developed semi-energy finite strip formulation [Ovesy et.al [10]]. It is emphasized that the developed semi-energy finite strip is based on a single term representation of the out-of-plane displacement.

$w=\mathrm{f}_{w} \sin \left(\frac{\mathrm{M} x}{2}\right)$

where

$$
\mathrm{f}_{w}=\left(1-3 \eta^{2}+2 \eta^{3}\right) w_{1}+b\left(\eta-2 \eta^{2}+\eta^{3}\right) \theta_{1}+\left(3 \eta^{2}-2 \eta^{3}\right) w_{2}+b\left(\eta^{3}-\eta^{2}\right) \theta_{2}
$$

and $\mathrm{M}=2 n \pi / L, \eta=y / b$ and $w_{1}, \theta_{1}, w_{2}, \theta_{2}$ are the undetermined out-of-plane nodal displacement parameters along edges 1 and 2 of the strip (see Figure 3). These nodal displacement parameters are also called the degrees of freedom (DOF). It is noted that $n$ represents the number of buckle half waves along the length of the strip. The out-of-plane displacement $w$ is then substituted into von Kármán's compatibility equation in order to find the corresponding in-plane displacement functions. In this process, the stress function $\mathrm{F}$ may be considered in two parts; one part varying periodically with $x$, and the other part constant with respect to $x$, i.e.

$$
\mathrm{F}=\mathrm{F}_{1}+\mathrm{F}_{2} \cos (\mathrm{M} x)
$$

Substituting F from Eq. 16 and $w$ from Eq. 15 in Eq. 13 and imposing the boundary conditions as Eq. 1, the in-plane $u$ displacement function corresponding to the assumed out-of-plane $w$ displacement can eventually be derived as Eq. 17.

$$
u=-\frac{\bar{u}}{L} x+\mathrm{f}_{u}(y) \sin (\mathrm{M} x)
$$

where

$$
\mathrm{f}_{u}(y)=\mathrm{f}_{u}=\frac{\mathrm{M}}{8}\left(\Psi^{\prime \prime}+v \mathrm{M}^{2} \Psi-\frac{\mathrm{f}_{w}^{2}}{2}\right)
$$

and $\Psi$ can be found from the following equation:

$$
\Psi^{\prime \prime \prime \prime}-2 \mathrm{M}^{2} \Psi^{\prime \prime}+\mathrm{M}^{4} \Psi=\left(\mathrm{f}_{w}^{\prime}\right)^{2}-\left(\mathrm{f}_{w} \mathrm{f}_{w}^{\prime \prime}\right)
$$

It is noted that the prime sign designates the derivatives of the corresponding parameter with respect to $\mathrm{y}$, thus, for example $\mathrm{f}_{w}^{\prime \prime}=\partial^{2} \mathrm{f}_{w} / \partial y^{2}$. It is also noted that the first term on the right hand 
side of Eq. 17 represents the prescribed uniform end shortening. The amplitude of the second term evaluated at $y=0$ and $y=b$ (i.e. $\left.\mathrm{f}_{u}\right|_{y=0}$ and $\left.\mathrm{f}_{u}\right|_{y=\mathrm{b}}$ ) represents the local degrees of freedom $u_{1}$ and $u_{2}$ respectively (see Figure 3). The solution of Eq. 19 is composed of two parts; the particular integral solution, and the complementary function solution. The particular integral solution depends on the function $\mathrm{f}_{w}$ only, thus it can be conveniently evaluated. The complementary function solution is as follows:

$$
\Psi_{\text {C.F. }}=\mathrm{B}_{1} \sinh (\mathrm{M} y)+\mathrm{B}_{2} \cosh (\mathrm{M} y)+\mathrm{B}_{3} y \sinh (\mathrm{M} y)+\mathrm{B}_{4} y \cosh (\mathrm{M} y)
$$

The coefficients $\mathrm{B}_{1} \rightarrow \mathrm{B}_{4}$ are unknown at present, but it is assumed that these coefficients and subsequently $\Psi_{\mathrm{C} . \mathrm{F}}$ and $\Psi$ are known so that the analysis to find the in-plane displacements can be completed. After substituting for the appropriate terms and simplification, the degrees of freedom $u_{1}$ and $u_{2}$ can be found explicitly in terms of $w_{1}, \theta_{1}, w_{2}, \theta_{2}, \mathrm{~b}, \mathrm{M}, v$ and $\mathrm{B}_{1} \rightarrow \mathrm{B}_{4}$. It is shown by Ovesy et al [10] that the in-plane displacement $v$ function can be derived as follow:

$v=v \frac{\bar{u}}{L} y+\mathrm{I}_{1 v}+\mathrm{f}_{v} \cos (\mathrm{M} x)-\left.\mathrm{f}_{v}\right|_{y=0}+\left.v\right|_{x=0, y=0}$

where

$$
\mathrm{f}_{\mathrm{v}}(y)=\mathrm{f}_{\mathrm{v}}=\frac{1}{8}\left[\Psi^{\prime \prime \prime}-(2+v) \mathrm{M}^{2} \Psi^{\prime}+\mathrm{f}_{w} \mathrm{f}_{w}^{\prime}\right]
$$

and

$$
\mathrm{I}_{1 \mathrm{v}}=-\int_{0}^{y}\left[\frac{v \mathrm{M}^{2}}{16} \mathrm{f}_{w}^{2}+\frac{\left(\mathrm{f}_{w}^{\prime}\right)^{2}}{4}\right] d y
$$

The above equation describes the in-plane $v$ displacement function corresponding to the assumed out-of-plane $w$ displacement function. The first term on the right hand side of Eq. 21 describes the transverse in-plane expansion of the strip, which occurs due to the Poisson's ratio effect. The second term (i.e. $\mathrm{I}_{1 \mathrm{v}}$ ) describes the transverse in-plane movement of the longitudinal fibers of the strip. This movement, which is constant along the length of a given fiber, varies from a minimum value of zero at edge $y=0$ to its maximum value at the edge $y=b$. The third term describes the in-plane waviness of the longitudinal fibers. The amplitude of this term evaluated at $y=0$ and $y=b$ (i.e. $\left.\mathrm{f}_{v}\right|_{y=0}$ and $\left.\mathrm{f}_{v}\right|_{y=b}$ ) represents the local degrees of freedom $v_{1}$ and $v_{2}$ respectively (see Figure 3). These degrees of freedom can explicitly be described in terms of $w_{1}, \theta_{1}, w_{2}, \theta_{2}, \mathrm{~b}, \mathrm{M}, v$ and $\mathrm{B}_{1}-\mathrm{B}_{4}$. Finally, the fourth term (which is equivalent to $v_{1}$ ) and the fifth term on the right hand side of Eq. 21 represent values which remain constant at all points on a given strip. The existence of the fourth term on the right hand side of Eq. 21 (i.e. $-\left.f_{v}\right|_{y=0}=-v_{1}$ ) allows the point $(x=0, y=0)$ to be treated as a reference point in terms of its deflection being connected to another strip or being restrained. It is noted that the function $\Psi$ is not yet fully known due to the coefficients $\mathrm{B}_{1} \rightarrow \mathrm{B}_{4}$ being unknown. These coefficients are obtained by treating them as unknowns, and solving for them while all other parameters, including all degrees of freedom (i.e. $u_{1}, v_{1}, w_{1}, \theta_{1}$, $u_{2}, v_{2}, w_{2}$ and $\theta_{2}$ ), are assumed to be known. Thus, the coefficients $\mathrm{B}_{1} \rightarrow \mathrm{B}_{4}$ are explicitly described in terms of $u_{1}, v_{1}, w_{1}, \theta_{1}, u_{2}, v_{2}, w_{2}, \theta_{2}, \mathrm{~b}, \mathrm{M}$ and $v$. Having found the coefficients $\mathrm{B}_{1} \rightarrow \mathrm{B}_{4}$, they are substituted in Eq. 20 to find the complementary function solution. Subsequently, the combination of the complementary function and particular integral solutions are used to substitute for $\Psi$ in the expressions describing the in-plane displacement $u$ (i.e. Eqs. 17-19) and $v$ (i.e. Eqs. 21-23). 
Having obtained the form of the in-plane displacements that correspond to the postulated form of the out-of-plane displacement, the membrane strain energy of the strip is now evaluated by using Eq. 11, and substituting for $\varepsilon$ in terms of the derivatives of the in-plane and out-of-plane displacements employing Eqs. 5, 15, 17 and 21. After performing the integration with respect to $x$ between 0 and $\mathrm{L}$, the following expression is obtained:

$$
\begin{aligned}
\mathrm{U}_{\mathrm{ms}}= & \frac{\mathrm{ELt}}{2} \int_{0}^{\mathrm{b}}\left\{\left(\frac{\overline{\mathrm{u}}}{\mathrm{L}}\right)^{2}+\frac{\mathrm{M}^{4}}{128}\left(\Psi^{\prime \prime}\right)^{2}+\frac{\mathrm{M}^{6}}{64} v \Psi \Psi^{\prime \prime}+\frac{\mathrm{M}^{8}}{128} \Psi^{2}+\right. \\
& \left.\frac{\mathrm{M}^{6}}{64}(1+v)\left(\Psi^{\prime}\right)^{2}+\frac{\mathrm{M}^{4}}{256} \mathrm{f}_{w}^{4}-\frac{\mathrm{M}^{2} \overline{\mathrm{u}}}{8 \mathrm{~L}} \mathrm{f}_{w}^{2}\right\} d y
\end{aligned}
$$

The bending strain energy $U_{b s}$ is expanded by substituting for $w$ from Eq. 15 into Eq. 12. Then, the total potential energy of the strip can be found by adding the membrane strain energy of the strip to the bending strain energy (see Eq. 8). The total potential energy for a strip is re-arranged in the form given below:

$$
V_{s}=U_{s}=\frac{1}{2} \mathrm{~d}_{\mathrm{s}}^{\mathrm{T}}\left(\mathrm{K}-\frac{\bar{u}}{l} \mathrm{~K}^{*}\right) \mathrm{d}_{\mathrm{s}}+\frac{1}{6} \mathrm{~d}_{\mathrm{s}}^{\mathrm{T}} \mathrm{K}_{1} \mathrm{~d}_{\mathrm{s}}+\frac{1}{12} \mathrm{~d}_{\mathrm{s}}^{\mathrm{T}} \mathrm{K}_{2} \mathrm{~d}_{\mathrm{s}}
$$

Here $\mathrm{K}, \mathrm{K}^{*}, \mathrm{~K}_{2}$ and $\mathrm{K}_{1}$ are symmetric square stiffness matrices. The coefficients of $\mathrm{K}$ and $\mathrm{K}^{*}$ are constant whilst those of $\mathrm{K}_{1}$ and $\mathrm{K}_{2}$ are linear and quadratic functions, respectively, of the displacements. The column matrix $\mathrm{d}_{\mathrm{s}}$ contains the strip degrees of freedom. In evaluating $V_{s}$, all the integrations in the $x$ and $y$ directions are determined analytically.

For the whole structure, comprising an assembly of finite strips, the total potential energy is simply the summation of the potential energies of the individual finite strips. Correspondingly, whole structure matrices which are equivalent of those appearing in Eq. 25 for the individual finite strip are generated by appropriate summations in the standard fashion. Thus, the potential energy for the whole structure can be expressed as:

$$
\bar{V}=\frac{1}{2} \overline{\mathrm{d}}^{\mathrm{T}}\left(\overline{\mathrm{K}}-\frac{\bar{u}}{l} \overline{\mathrm{K}}^{*}\right) \overline{\mathrm{d}}+\frac{1}{6} \overline{\mathrm{d}}^{\mathrm{T}} \overline{\mathrm{K}}_{1} \overline{\mathrm{d}}+\frac{1}{12} \overline{\mathrm{d}}^{\mathrm{T}} \overline{\mathrm{K}}_{2} \overline{\mathrm{d}}
$$

where the overbar indicates a whole-structure quantity.

The structure equilibrium equations are obtained by applying the principle of minimum potential energy. The partial differentiation of the structure potential energy with respect to each degree of freedom in turn gives a set of non-linear equilibrium equations as outlined below:

$$
\left(\overline{\mathrm{K}}-\frac{\bar{u}}{l} \overline{\mathrm{K}}^{*}+\frac{1}{2} \overline{\mathrm{K}}_{1}+\frac{1}{3} \overline{\mathrm{K}}_{2}\right) \overline{\mathrm{d}}=0 \quad \text { or } \quad \overline{\mathrm{K}}_{\mathrm{s}} \cdot \mathrm{d}=0
$$

where $\overline{\mathbf{K}}_{\mathrm{S}}$ is the global/structural stiffness matrix, and $\overline{\mathrm{d}}$ is a vector, which includes the degrees of freedom for the whole structure. The latter set of equations needs to be modified by applying the appropriate boundary conditions. These equations can be solved using an iterative procedure and here it is the Newton-Raphson procedure that is used, in the manner described below. 
It is assumed that $\overline{\mathrm{d}}_{\mathrm{i}}$ is an approximate trial solution at a particular prescribed end shortening $\bar{u}$. In this case, $F\left(\overline{\mathrm{d}}_{\mathrm{i}}\right)$, which is defined by the following equation, denotes the error in the approximation:

$$
\mathrm{F}\left(\overline{\mathrm{d}}_{\mathrm{i}}\right)=\overline{\mathrm{K}}_{\mathrm{s}} \cdot \overline{\mathrm{d}}_{\mathrm{i}} \neq 0
$$

Applying the Newton-Raphson algorithm to this trial solution, an improved solution $\overline{\mathrm{d}}_{\mathrm{i}+1}$ is obtained by equating to zero the curtailed Taylor's expansion of $F\left(\bar{d}_{i+1}\right)$ in the neighborhood of $\bar{d}_{i}$, i.e.

$$
\mathrm{F}\left(\overline{\mathrm{d}}_{\mathrm{i}+1}\right) \cong \mathrm{F}\left(\overline{\mathrm{d}}_{\mathrm{i}}\right)+\frac{\partial \mathrm{F}\left(\overline{\mathrm{d}}_{\mathrm{i}}\right)}{\partial \overline{\mathrm{d}}_{\mathrm{i}}}\left(\overline{\mathrm{d}}_{\mathrm{i}+1}-\overline{\mathrm{d}}_{\mathrm{i}}\right)=0
$$

Differentiating Eq. 28 with respect to $\overline{\mathrm{d}}_{\mathrm{i}}$ gives

$$
\frac{\partial \mathrm{F}\left(\overline{\mathrm{d}}_{\mathrm{i}}\right)}{\partial \overline{\mathrm{d}}_{\mathrm{i}}}=\overline{\mathrm{K}}-\frac{\bar{u}}{l} \overline{\mathrm{K}}^{*}+\overline{\mathrm{K}}_{1}+\overline{\mathrm{K}}_{2}=\overline{\mathrm{K}}_{\mathrm{T}}
$$

where $\overline{\mathrm{K}}_{\mathrm{T}}$ is the symmetric tangent stiffness matrix evaluated at $\overline{\mathrm{d}}_{\mathrm{i}}$. Eq. 29 can now be expressed as

$$
\overline{\mathrm{K}}_{\mathrm{T}} \Delta \overline{\mathrm{d}}_{\mathrm{i}}=-\mathrm{F}\left(\overline{\mathrm{d}}_{\mathrm{i}}\right)
$$

where $\Delta \overline{\mathrm{d}}_{\mathrm{i}}$ is the correction at the $i$ th iteration. Having obtained the solution of this equation for $\Delta \overline{\mathrm{d}}_{\mathrm{i}}$, the improved solution at the end of the $i$ th iteration for a prescribed value of $\bar{u}$, is then given by

$$
\overline{\mathrm{d}}_{\mathrm{i}+1}=\overline{\mathrm{d}}_{\mathrm{i}}+\Delta \overline{\mathrm{d}}_{\mathrm{i}}
$$

In the present study, the iterative procedure is repeated until the following convergence criteria are satisfied:

$$
\sqrt{\frac{\sum \Delta \overline{\mathrm{d}}_{\mathrm{i}}^{2}}{\sum \overline{\mathrm{d}}_{\mathrm{i}+1}^{2}}}<10^{-7}
$$

and

$$
\sqrt{\sum \mathrm{F}_{i}^{2}}<10^{-5}
$$

where the summation relates to all active degrees of freedom, and $F_{i}=F\left(\bar{d}_{i}\right)$.

Once the global equilibrium equations are solved and the nodal degrees of freedom are found for a particular prescribed end shortening, it is possible to calculate the out-of-plane displacement $w$ in any finite strip using Eq. 15 directly. The calculation of the in-plane displacements $u$ and $v$ is achieved after finding $\mathrm{I}_{10}, \Psi$ and the derivatives of $\Psi$.

The stresses and strains at any point in any strip are calculated using Eqs. 3 to 6 . 
The longitudinal force/load acting on a strip at a given cross-section along the strip length $\mathrm{P}_{\mathrm{s}}$ is determined by integrating the longitudinal mid-plane stresses $\sigma_{x}$ over the strip cross-sectional area, i.e.

$$
\mathrm{P}_{s}=-\int_{0}^{l} \int_{0}^{\mathrm{b}} \sigma_{x} d x d y
$$

The total longitudinal force/load acting on a plate or plate structures at a given cross section along their length, corresponding to a prescribed end shortening, is obtained by summation of all strip forces $\mathrm{P}_{\mathrm{s}}$ (given by Eq. 35 ) at the same cross section, i.e.

$$
\mathrm{P}=\sum \mathrm{P}_{\mathrm{s}}
$$

It is emphasized that the positive $\mathrm{P}$ values represent compressional loads.

\subsection{Theoretical Developments of Full-energy Finite Strip}

Unlike the semi-energy method, which has been developed based on a single term concept only, the full-energy finite strip method employs a multi-term concept. The main difference between the two methods, however, lies in the fact that in the full-energy method, in addition to postulating a deflected form for the out-of-plane displacement, the in-plane displacements of the finite strip are also postulated by the appropriate deflected forms from the commencement of analysis. As far as the expression of the in-plane displacements and out-of-plane displacement in the full-energy method are concerned, in line with the work of Dawe et al. [5], the following forms are assumed:

$$
\begin{aligned}
& u=-\frac{\bar{u}}{L} x+\sum_{n=1}^{r u} \mathbf{f}_{n}^{\mathrm{u}} \sin \left(\frac{n \pi x}{L}\right) \\
& v=v \frac{\bar{u}}{L} y+\sum_{n=0}^{r v} \mathrm{f}_{n}^{\mathrm{v}} \cos \left(\frac{n \pi x}{L}\right) \\
& w=\sum_{n=1}^{r w} \mathrm{f}_{\mathrm{w} n} \sin \left(\frac{n \pi x}{L}\right)
\end{aligned}
$$

where the $\mathrm{f}_{n}^{\mathrm{u}}, \mathrm{f}_{n}^{\mathrm{v}}$ and $\mathrm{f}_{\mathrm{w} n}$ are the appropriate transverse polynomial interpolation functions, involving undetermined nodal parameters. These functions are expressed below:

$$
\begin{aligned}
& \mathrm{f}_{n}^{u}(y)=\frac{1}{2}(1-2 \eta) u_{1 n}+\frac{1}{2}(1+2 \eta) u_{2 n} \\
& \mathrm{f}_{n}^{\mathrm{v}}(y)=\frac{1}{2}(1-2 \eta) v_{1 n}+\frac{1}{2}(1+2 \eta) v_{2 n} \\
& \mathrm{f}_{w n}=\left(1-3 \eta^{2}+2 \eta^{3}\right) w_{1 n}+b\left(\eta-2 \eta^{2}+\eta^{3}\right) \theta_{1 n}+\left(3 \eta^{2}-2 \eta^{3}\right) w_{2 n}+b\left(\eta^{3}-\eta^{2}\right) \theta_{2 n}
\end{aligned}
$$


where $\eta=y / b$ and the $u_{1 n}, u_{2 n}, v_{1 n}, v_{2 n}, w_{1 n}, \theta_{1 n}, w_{2 n}, \theta_{2 n}$ represent the degrees of freedom corresponding to the $n$th series' term along the length of the strip. It is seen that for a given term, the shape function $f_{\mathrm{w} n}$ is the same as that already used in connection with the semi-energy method (i.e. Eq. 15-b).

With the establishment of the finite strip displacement fields according to the above-mentioned equations, the rest of the analysis is carried out in the same manner as that already described with respect to semi-energy finite strip method. It is worth mentioning that in evaluating the potential energy of the full-energy strip, similar to the process followed for the semi-energy finite strip, all integrations in the $x$ and $y$ directions are determined analytically. Further details regarding theoretical developments of the full-energy finite strip approach are given in an earlier publication by Ovesy and Ghannadpour [13].

\section{RESULTS AND DISCUSSIONS}

The type of plate structure investigated is plain I-section struts whose cross-sectional geometry is depicted in Figure 1. It is noted that the present study is concerned exclusively with local buckling. Considering the fact that the transverse in-plane displacement $v$ and the out-of-plane displacement $\mathrm{w}$ are characterized by differing trigonometric series, it is further assumed that the in-plane and out-of-plane deflections at junctions are uncoupled. The plate components are assumed to be free to wave across their width along the junctions where the out-of-plane deflection of each of the plates vanishes separately i.e. $\sigma_{y}=0, w=0$ along the junctions. This assumption is a valid approximation for an initial post-local-buckling analysis. It is noted that it is neither possible nor necessary to define a global co-ordinate system for the plate structure as a whole due to the uncoupling between $v$ and $w$ displacements at junctions. Hence, within any component plate the local degrees of freedom in the interior can be used directly to relate to the structure. Since the local buckling mode of I-section strut is symmetric with respect to the axis passing through web centre parallel to the flanges, it was sufficient to analyze one half of the cross-section while the necessary symmetric boundary conditions were applied along the axis of symmetry. In order to reflect the symmetry of the problem in the longitudinal direction, the appropriate series' terms were chosen and used in the full-energy finite strip approach. That is to say, for $u$ the first three even terms $(n=$ $2,4,6)$ and for $v$ the first four even terms $(n=0,2,4,6)$ and for $w$ the first two odd terms $(n=1,3)$ were used. It is noted that in the semi-energy finite strip approach, only one term $(n=1)$ was used for $w$.

The number of strips $N_{s}$ which is needed for convergence is examined. For example, in the full energy method and for an I-section strut with a uniform thickness across the section and the strut length equal to the natural half-wave length with $b_{f} / b_{w}=0.4$, the post-buckling compressional stiffness ratio $\mathrm{S}^{*} / \mathrm{S}$ is equal to $0.6333,0.6319$ and 0.6314 for the number of strips $N_{S}$ equal to 24,36 and 48 respectively. Thus in the full energy method, the strips allocation corresponding to 16 strips to the half-web and 32 strips to the flange, resulting in a total of 48 strips for half the section is considered adequate. The same study resulted in a total of 24 strips in the semi-energy approach by assigning 8 identical strips to each of the plate elements meeting at the junction. It is worth mentioning that in presenting the results, the Poisson's ratio $v$ is assumed to be 0.3 and a common Young's modulus with the value within the range corresponding to steel module (i.e. $E=207000$ $\mathrm{MPa}$ ) was assumed for I-section. Figures 4 and 5 depict the initial post-buckling ( $\mathrm{S}^{*}$ ) to pre-buckling (S) compressional stiffness ratio (i.e. $\mathrm{S}^{*} / \mathrm{S}$ at the instant of local instability) for a number of I-section struts with a variety of geometric configurations. It is noted that the 
post-buckling compressional stiffness of the strut is defined by $\mathrm{S}^{*}=\partial \mathrm{P} / \partial \overline{\mathrm{u}}$, where $\mathrm{P}$ is the total longitudinal load of the struts. It is also noted that the pre-buckling compressional stiffness (i.e. $\mathrm{S}=\partial \mathrm{P} / \partial \overline{\mathrm{u}})$ is a constant value. In Figure 4, the strut length is equal to the natural half-wave lengths, whereas in Figure 5, the strut length is constant and equal to the web width. Moreover, In Figure 4, a uniform thickness across the section is assumed, whereas in Figure 5 three different flange-web thickness ratios are considered. It is worth mentioning that in Figure 4, the finite strip analyses were carried out only for those configurations that are marked with symbols on the graph. The smoothest curve was then passed through the points to show the general behaviour of the structures.

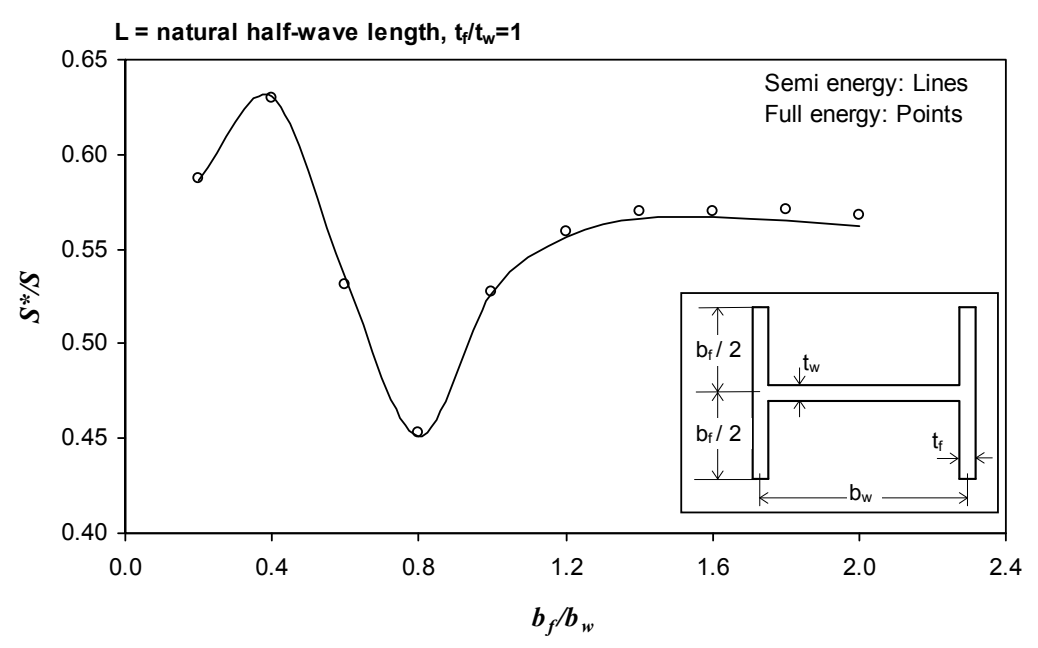

Figure 4. Post-Buckling Compressional Stiffness Ratio of an I-Section Strut at the Instant of Buckling

In Figure 5, however, over 200 different combinations of shape factors were analysed. Both figures indicate that for each curve, there is a particular shape factor which produces a minimum $\mathrm{S}^{*} / \mathrm{S}$ ratio. Prior to this shape factor, buckling is initiated in the web which is being restrained by the flanges against further deflection. Thereafter, the flanges start the buckling and the web restrains them. It is seen in Figure 5 that prior to the shape factor corresponding to the minimum $\mathrm{S}^{*} / \mathrm{S}$ ratio, where buckling is initiated in the web, the lower the $t_{f} / t_{w}$ ratio, the lesser the post-buckling stiffness. However, thereafter, where flange predominates buckling, the lower the $t_{f} / t_{w}$ ratio, the higher the $\mathrm{S}^{*} / \mathrm{S}$ values. This behaviour may be attributed to the fact that for web buckling, the thick flange provides a high degree of restraint on the thin web prior to and after the local buckling, causing an increase in the post-buckling compressional stiffness. However, when buckling is dominant in the flange, the thin web is incapable of imposing such restraints and consequently a substantial reduction in the stiffness is experienced. It is seen in both figures that the general agreement between the results obtained by the semi-energy approach and those obtained by the full-energy method are very good. It is also seen that when the flange gets thinner, i.e. the cases with $\mathrm{t}_{\mathrm{f}} / \mathrm{t}_{\mathrm{w}}=0.5$ in Figure 5 which are also tabulated in Table 1, and when the flange gets longer, i.e. the cases with higher $b_{f} / b_{w}$ ratios in Figure 4 which correspond to longer natural half-wave length, the increase in $b_{f} / b_{w}$ ratio has caused the semi-energy results to be more accurate by predicting lower values for post-buckling compressional stiffness compared to those achieved by full-energy method. This difference may be attributed to the fact that for the aforementioned cases, where the buckling of the section is highly dominated by that of the flange which is effectively free on both sides, the formulation of the semi-energy method in which the in-plane displacements are obtained by solving the von Kármán's compatibility equation is more accurate. Figure 6 represents 
the non-dimensional load-end shortening variation for struts with flange-web width ratio equal to 0.5. The length of the struts is assumed to be equal to their web width. It is noted that three different $\mathrm{t}_{\mathrm{f}} / \mathrm{t}_{\mathrm{w}}$ ratios are studied. The non-linear nature of the behaviour, which happens due to the effect of change in the buckle shape, can clearly be seen for all cases. Once again, a very good agreement is seen to exist between the results obtained by the semi-energy approach and those obtained by the full-energy method. It is to be noted that in the current semi-energy approach only one term is utilized, whereas several terms are implemented in the formulation of the full-energy method. Therefore, it is expected that the accuracy of the semi-energy approach will improve if more than one term is utilized in its formulation.

Table 1. Comparison of the Semi Energy Results $\left(S^{*} / S\right)$ with the Results of Full Energy

\begin{tabular}{c|c|c|c}
\hline \multirow{2}{*}{$\mathbf{b}_{\mathbf{f}} / \mathbf{b}_{\mathbf{w}}$} & \multicolumn{2}{|c|}{$\mathbf{s}^{*} / \mathbf{S}$} & \multirow{2}{*}{ Error (\%) } \\
\cline { 2 - 3 } & Semi energy & Full energy & \\
\hline 2.0 & 0.6615 & 0.6694 & 0.79 \\
1.8 & 0.6766 & 0.6826 & 0.60 \\
1.6 & 0.6930 & 0.6971 & 0.41 \\
1.4 & 0.7105 & 0.7127 & 0.22 \\
1.2 & 0.7284 & 0.7289 & 0.05 \\
1.0 & 0.7433 & 0.7443 & 0.10 \\
0.8 & 0.7356 & 0.7390 & 0.34 \\
0.7 & 0.6855 & 0.6872 & 0.17 \\
0.6 & 0.5223 & 0.5257 & 0.34 \\
0.5 & 0.4277 & 0.4329 & 0.52 \\
0.4 & 0.4928 & 0.4960 & 0.32 \\
0.2 & 0.5027 & 0.5052 & 0.25 \\
\hline
\end{tabular}

Figure 7 depicts the longitudinal mid-plane stress distributions across the cross-section of the struts with $t_{f} / t_{w}$ ratio equal to 1 , which is investigated earlier in connection with the load-end shortening variation with reference to Figure 6 . The stress variation is presented at the crest of the buckle at the load levels equal to twice and three times the buckling load. Once again due to the symmetry, the stress distributions are shown for only a half of the cross-section. A good agreement is seen to exist among the results obtained by both finite strip methods.

As expected, the maximum membrane stress occurs at the plate junction at the crest of the buckle for any specified level of loading. The figure show that, at the load levels under consideration, the flanges and the web do not apply any significant restraint to each other at the junction. It is also evident that the flanges and the webs experience a considerable amount of stress loss, particularly at load equal to $3 \mathrm{Pc}$, across their cross-section. The stress loss occurs to such an extent that the stresses become tensile at the flanges free edges. 


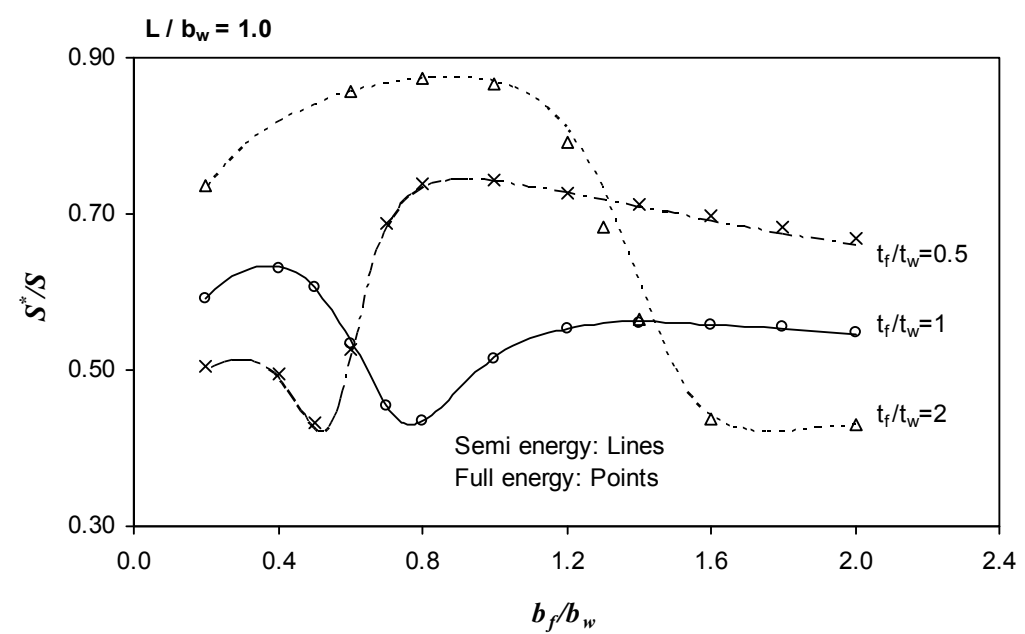

Figure 5. Post-Buckling Compressional Stiffness Ratio of I-Section Struts at the Instant of Buckling

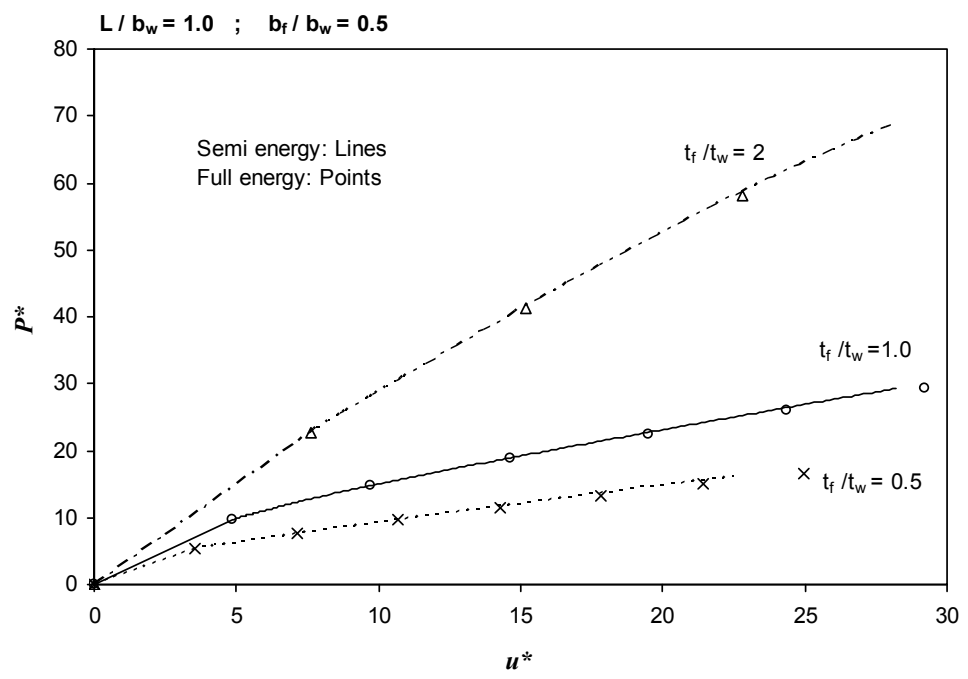

Figure 6. Load-Displacement Variation for I-Section Struts
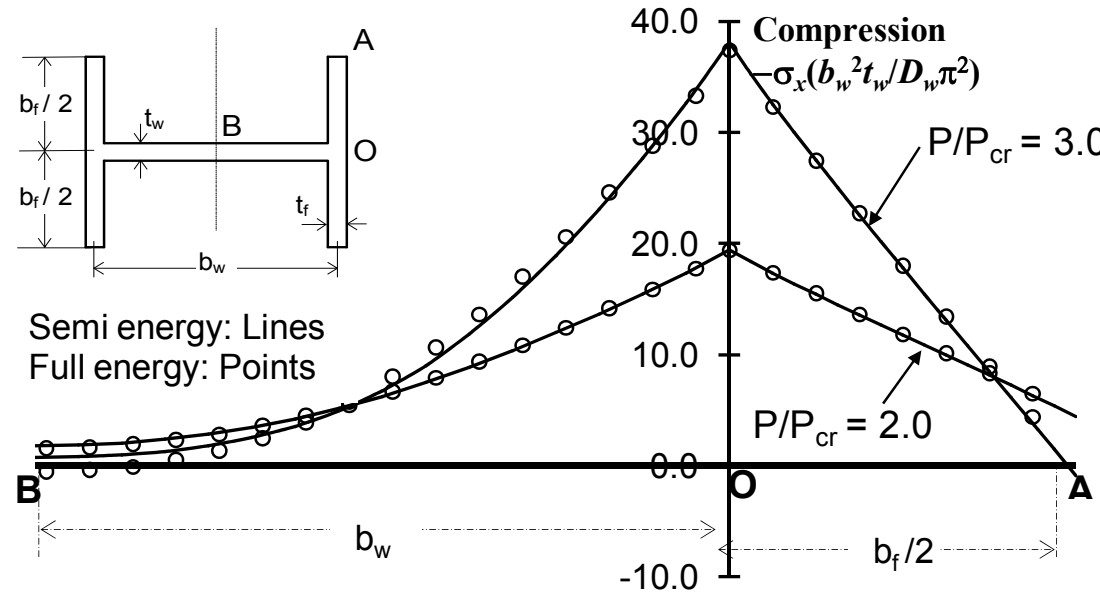

Figure 7. Longitudinal Membrane Stress Distribution at the Crest of the Buckle for I- Section Struts 


\section{CONCLUSIONS}

Two finite strip methods, namely the full-energy and the semi-energy FSM, are developed for predicting the geometrically non-linear response of I-sections with simply supported ends when subjected to uniform end shortening in their plane. Although the formulations of both finite strip methods are based on the concept of the principle of minimum potential energy, the first finite strip method utilizes a semi-energy finite strip, whereas in the second finite strip method (which is designated by the name full-energy finite strip method) all the displacements are postulated by the appropriate shape functions. The developed finite strip methods are then applied to analyze the post-local-buckling behavior of some representative I-sections. Through the comparison of the results, it is observed that for the geometries of the structure under study, the agreement between the results obtained by the semi-energy approach and those obtained by the full-energy method are very good.

However, it is expected that by implementing more than one term in the formulation of the semi-energy approach, and thus by allowing the structure to take up more flexibility if required, the accuracy of the semi-energy approach will improve.

\section{REFERENCES}

[1] Graves Smith, T.R. and Sridharan, S., "A Finite Strip Method for the Post-Locally-Buckled Analysis of Plate Structures”, Int. J. Mech. Sci., 1978, Vol. 20, pp. 833-842.

[2] Sridharan, S., "A Finite Strip Analysis of Locally Buckled Plate Structures subject to Nonuniform Compression", Eng. Struct., 1982, Vol. 4, pp. 249-255.

[3] Graves Smith, T. R. and Sridharan, S.,"The Local Collapse of Elastic Thin Walled Columns", J. STRUCT. MECH., 1980, Vol. 8, No. 4, pp. 471-489.

[4] Hancock, G. J., "Nonlinear Analysis of Thin Sections in Compression", Journal of the Structural Division, ASCE, 1981, Vol. 107, No. ST3, pp. 455-471.

[5] Dawe, D. J., and Lam, S. S. E. and Azizian, Z. G., "Finite Strip Post-Local-Buckling Analysis of Composite Prismatic Plate Structures", Computers \& Structures, 1993, Vol. 48, No. 6, pp. 1011-1023.

[6] Kwon, Y. B. and Hancock, G. J., "Post-Buckling Analysis of Thin-Walled Channel Sections Undergoing Local and Distortional Buckling", Research Report, No. R650, School of Civil and Mining Engineering, The University of Sydney, Australia, 1992.

[7] Azhari, M. and Bradford, M. A., "The Use of Bubble Functions for the Post-Local Buckling of Plate Assemblies by the Finite Strip Method”, Int. J. Numer. Meth. Engng., 1995, Vol. 38, pp. 955-968.

[8] Rhodes, J. and Harvey, J. M., "Examination of Plate Post-Buckling Behaviour", J. of Eng. Mech. Div. ASCE, 1977, Vol. 103, No. EM3, pp. 461-478.

[9] Marguerre, K., "The Apparent Width of Plates in Compression", NACA Technical Memorandum No. 833, National Advisory Committee for Aeronautics, Washington, D.C., 1937.

[10] Ovesy, H. R., and Loughlan J., and GhannadPour, S. A. M., "Geometric Non-Linear Analysis of Thin Flat Plates Under End Shortening, Using Different Versions of the Finite Strip Method", International Journal of Mechanical Sciences, 2005, Vol. 47, pp. 1923-1948.

[11] Ovesy, H. R., and Loughlan J., and GhannadPour, S. A. M., "Geometric Non-Linear Analysis of Channel Sections Under End Shortening, Using Different Versions of the Finite Strip Method", Computers \& Structures, 2006, Vol. 84, pp. 855-872. 
[12] Ovesy, H. R., and Loughlan J., and GhannadPour, S. A. M., and Morada, G., "Geometric Non-Linear Analysis of Box Sections Under End Shortening, Using Three Different Versions of the Finite Strip Method", Thin Walled Structures, 2006, Vol. 44, pp. 623-637.

[13] Ovesy, H. R., GhannadPour, S. A. M., "Geometric Nonlinear Analysis of Imperfect Composite Laminated Plates, Under End Shortening and Pressure loading, Using Finite Strip Method", Composite Structures, 2006, Vol. 75, pp. 100-105. 